\title{
Radical Cascade Multicomponent Minisci Reactions with Diazo
}

\section{Compounds}

Yong-Liang Su ${ }^{\ddagger}$, , Geng-Xin Liu ${ }^{\ddagger}$, , Luca De Angelis ${ }^{\ddagger, a}, \mathrm{Ru} \mathrm{He}^{\mathrm{a}}$, Ammar Al-Sayyed ${ }^{\mathrm{a}}$, Kirk S. Schanze ${ }^{\mathrm{a}}$, Wen-Hao Hu${ }^{\mathrm{b}}$, Huang Qiu*,b and Michael P. Doyle ${ }^{*, a}$

${ }^{a}$ Department of Chemistry, The University of Texas at San Antonio One UTSA Circle, San Antonio, TX 78249, USA

${ }^{\mathrm{b}}$ Guangdong Key Laboratory of Chiral Molecule and Drug Discovery, School of Pharmaceutical Sciences, Sun Yat-sen University, Guangzhou 510006, China

Email: qiuhuang@mail.sysu.edu.cn; michael.doyle@utsa.edu

\section{Contents}

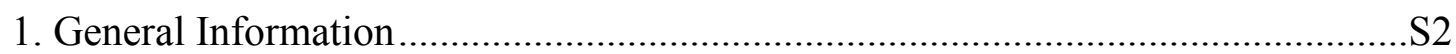

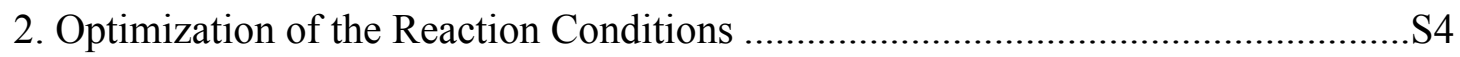

3. General Procedure and Characterization of Products ............................................S7

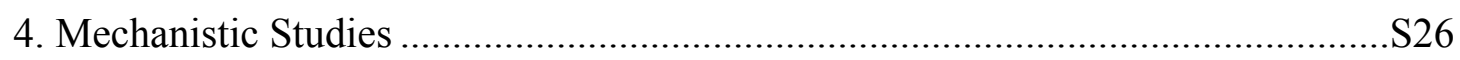

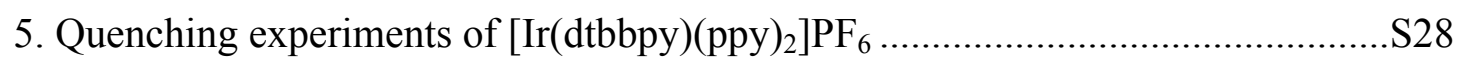

6. Crystallographic Data and Structure Refinement for Compounds $\mathbf{4 j}$ and $\mathbf{8 q}$........S31

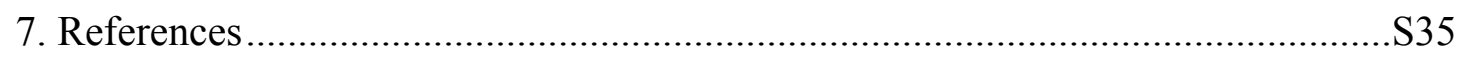

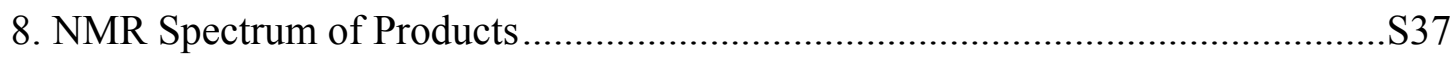

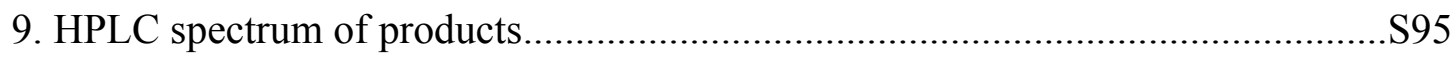




\section{General Information}

All reactions were carried out under an inert atmosphere $\left(\mathrm{N}_{2}\right)$ unless otherwise stated, with oven-dried glassware using standard techniques. Photocatalysts, ethyl diazoacetate (EDA) 2a and anhydrous solvents were purchased from Aldrich or Fisher. Heteroarenes $\mathbf{1 b}^{1 \mathrm{a}}, \mathbf{1} \mathbf{c}^{1 \mathrm{~b}}, \mathbf{1} \mathbf{d}^{1 \mathrm{c}}, \mathbf{1} \mathbf{h}^{1 \mathrm{~b}}, \mathbf{1 i}^{1 \mathrm{~d}}, \mathbf{1} \mathbf{m}^{1 \mathrm{e}}$ were prepared using the reported literature procedures. Other heteroarenes were purchased from Fisher and used without further purification.

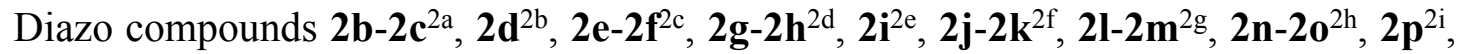
$\mathbf{2} \mathbf{q}^{2 \mathrm{j}}$ and $\mathbf{2} \mathbf{r}^{2 \mathrm{k}}$ were prepared using reported procedures. Alkene compounds $\mathbf{3} \mathrm{l}^{3 \mathrm{a}}, \mathbf{3} \mathbf{m}$ -

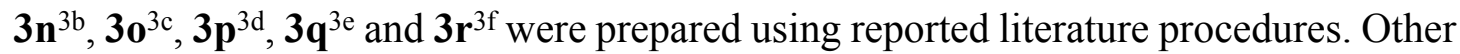
alkene compounds were purchased from Fisher or TCI and used without further purification. The photoreactor, equipped with a fan to keep the reactor at ambient temperature during the reaction processes, used two blue LED lamps (Kessil PR160L$467 \mathrm{~nm}, 50 \%$ intensity) approximately $8.5 \mathrm{~cm}$ away. Analytical thin-layer chromatography (TLC) was performed using glass plates pre-coated with 200-300 mesh silica gel impregnated with a fluorescent indicator $(254 \mathrm{~nm})$ or by dipping the plates either into a 5\% phosphomolybdate solution followed by heating or into an aqueous potassium permanganate solution. Column chromatography was performed on CombiFlash $^{\circledR}$ Rf200 and Rf+ purification systems using normal phase disposable columns. Melting points were obtained uncorrected from an Electro Thermo Mel-Temp DLX 104 device. All NMR spectra were recorded in $\mathrm{CDCl}_{3}$ on a Bruker spectrometer at $500 \mathrm{MHz}\left({ }^{1} \mathrm{H} \mathrm{NMR}\right)$ and $126 \mathrm{MHz}\left({ }^{13} \mathrm{C} \mathrm{NMR}\right)$. Chemical shifts were reported in ppm and calibrated using $\mathrm{CDCl}_{3}\left(7.26 \mathrm{ppm}\right.$ for ${ }^{1} \mathrm{H}, 77.16 \mathrm{ppm}$ for $\left.{ }^{13} \mathrm{C}\right)$ as standards, and coupling constants $(J)$ were given in hertz $(\mathrm{Hz})$. The peak information was described as: $\mathrm{br}=$ broad, $\mathrm{s}=$ singlet, $\mathrm{d}=$ doublet, $\mathrm{t}=$ triplet, $\mathrm{q}=$ quartet, $\mathrm{dd}=$ doublet of doublet, $\mathrm{m}=$ multiplet, and comp $=$ composite of magnetically non-equivalent protons. High-resolution mass spectra (HRMS) were performed on a Bruker MicroTOF-ESI mass spectrometer with an ESI source using a CsI or LTQ ESI positive ion calibration solution as the standard.

\section{Heteroarenes included in the manuscript}<smiles>Cc1ccnc2ccccc12</smiles>

1a<smiles>c1ccc(-c2ccnc3ccccc23)cc1</smiles>

$1 b$<smiles>CCCc1ccnc2ccccc12</smiles>

1c<smiles>Clc1ccnc2ccccc12</smiles>

1d<smiles>COc1ccc2c(Cl)ccnc2c1</smiles>

$1 \mathrm{e}$<smiles>Cc1ccc2ccccc2n1</smiles>

$1 f$<smiles>Cc1ccc2cc(F)ccc2n1</smiles>

$1 \mathrm{~g}$<smiles>Cc1cc2ccccc2cn1</smiles>

11<smiles>Cc1cncc2ccccc12</smiles>
$1 \mathrm{~m}$<smiles>COC(=O)c1cncc2ccccc12</smiles>
$1 n$<smiles>COc1cccc2cnccc12</smiles><smiles>Clc1cccc2cnccc12</smiles>

10

$1 i$

1j<smiles>Cc1ccc2cnccc2c1</smiles>

$1 q$<smiles>c1ccc2c(c1)cnc1ccccc12</smiles>

$1 r$<smiles>CCC1=CN2CCC([C@@H](O)c3ccnc4ccc(OC)cc34)CC2C1</smiles>

$1 \mathrm{~s}$<smiles>Cn1cnc2ccccc21</smiles>

$1 t$<smiles>CCOC(=O)c1c(C)ccnc1C</smiles>

14 
Diazo compounds included in the manuscript

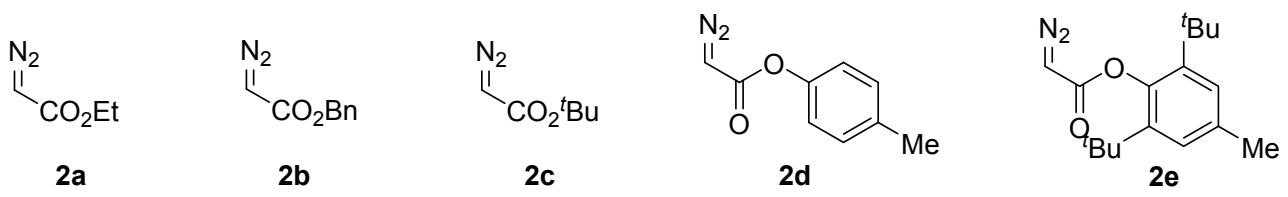<smiles>C#CCCCOC(=O)C=N</smiles>

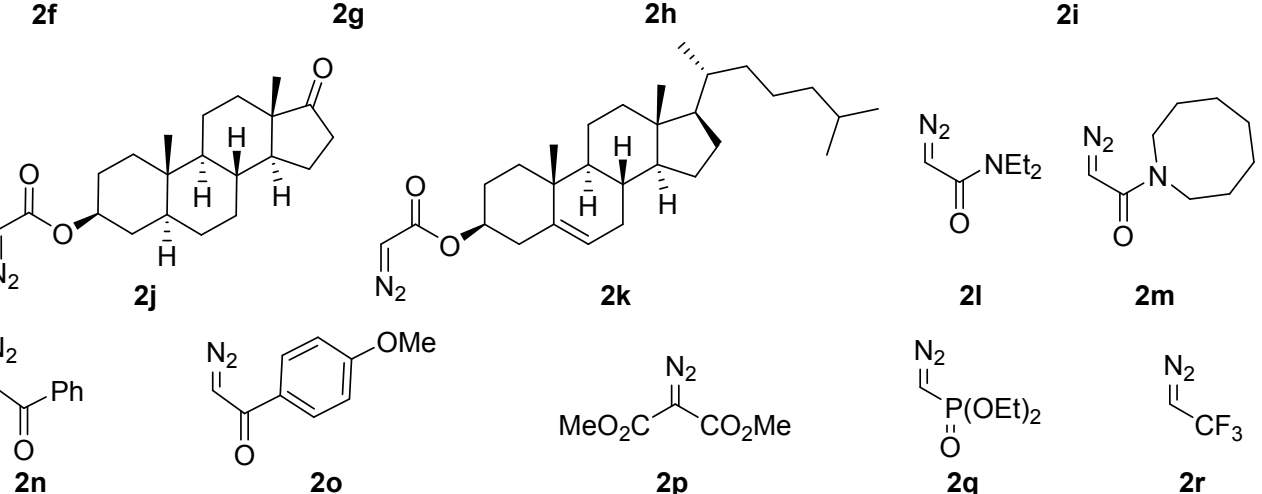

Alkenes included in the manuscript

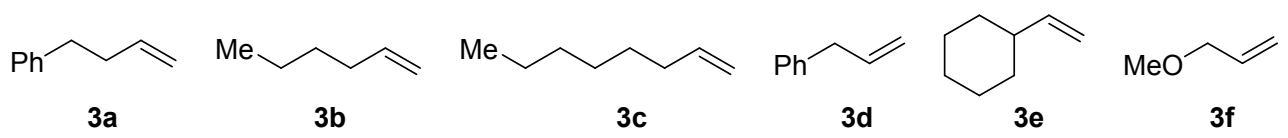

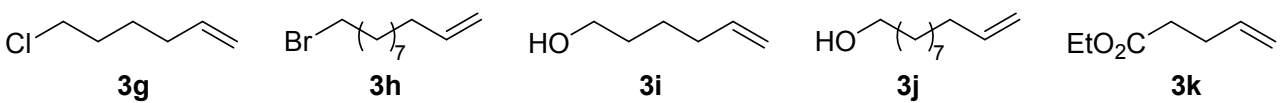<smiles>C=CCCCCOC(=O)c1cccs1</smiles><smiles>C=CCCCOc1ccc2c(c1)CCC1C2CCC2C1CCC1(C)C(=O)CCC21</smiles>

Limitations: either no or only trace amount of product

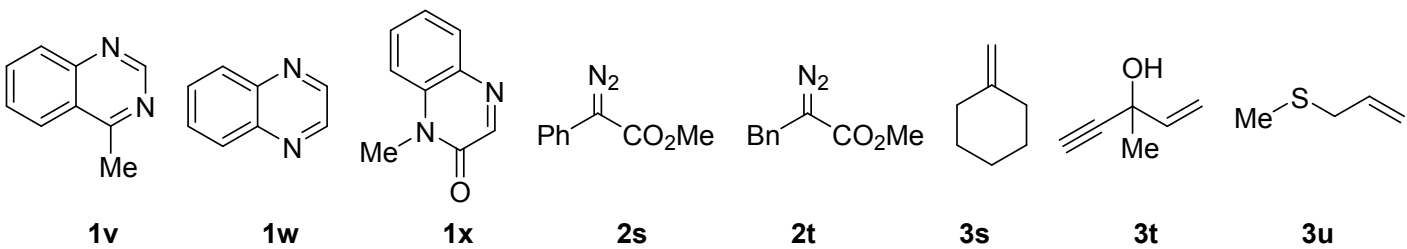




\section{Optimization of the Reaction Conditions}

Table S1. Investigation of the addition time of $\mathbf{2 a}$ for the multicomponent reaction. ${ }^{a}$
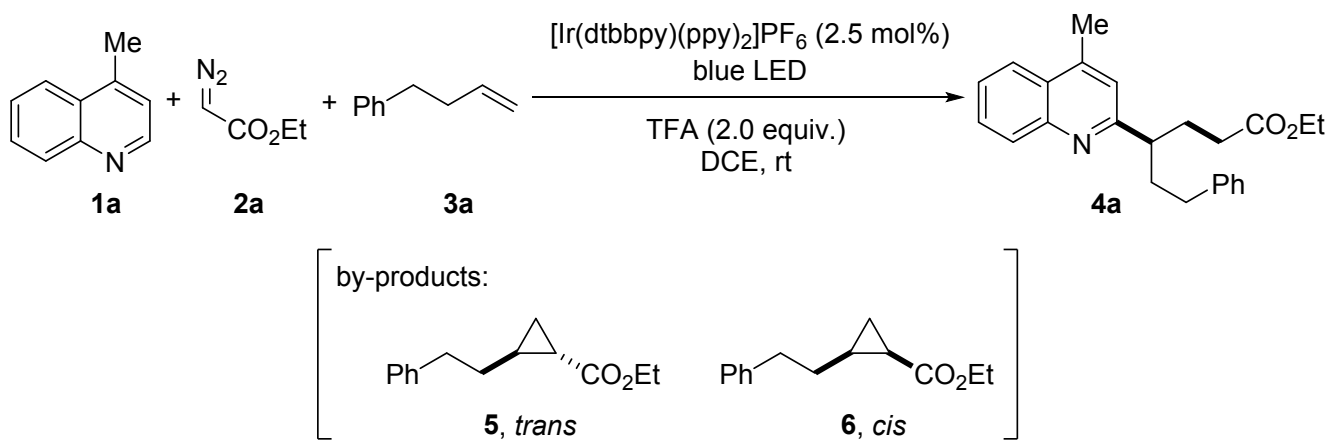

\begin{tabular}{ccc:ccc} 
entry & addition time of $\mathbf{2 a}(\mathrm{h})$ & $\mathbf{4 a / 5 / 6}(\%)^{b}$ & entry & addition time of $\mathbf{2 a}(\mathrm{h})$ & $\mathbf{4 a / 5 / 6}(\%)^{b}$ \\
\hline 1 & $25 / 8 / 8$ & 3 & 5 & $47 / 5 / 5$ \\
2 & 1 & $34 / 6 / 6$ & $4^{c}$ & 5 & $34 / 5 / 5$
\end{tabular}

${ }^{a}$ Conditions: 1a $(0.2 \mathrm{mmol}, 1.0$ equiv. $)$, [ $\left.\operatorname{Ir}(\mathrm{dtbbpy})(\mathrm{ppy})_{2}\right] \mathrm{PF}_{6}(2.5 \mathrm{~mol} \%), 3 \mathbf{3}(0.4 \mathrm{mmol}, 2.0$ equiv. $), 2 \mathrm{a}$ (0.4 mmol, 2.0 equiv.), TFA ( 0.4 mmol, 2.0 equiv.), DCE $(2.0+1.0 \mathrm{~mL})$, rt, blue LED (Kessil PR160L$467 \mathrm{~nm}, 50 \%$ intensity), $18 \mathrm{~h}$. The solution of $2 \mathbf{a}$ in DCE $(1.0 \mathrm{~mL})$ was added to the reaction mixture via a syringe pump over the indicated time. ${ }^{b}$ The yields of $\mathbf{4 a} / \mathbf{5} / \mathbf{6}$ were determined by ${ }^{1} \mathrm{H}$ NMR spectroscopic analyses of the reaction mixture using $\mathrm{CHBr}_{3}$ as the internal standard. ${ }^{c}$ The solution of $\mathbf{2 a}$ and $\mathbf{3 a}$ in DCE $(1.0 \mathrm{~mL})$ was added to the reaction mixture via a syringe pump over $5 \mathrm{~h}$.

Table S2. Investigation of the most suitable acid for the multicomponent reaction. ${ }^{a}$

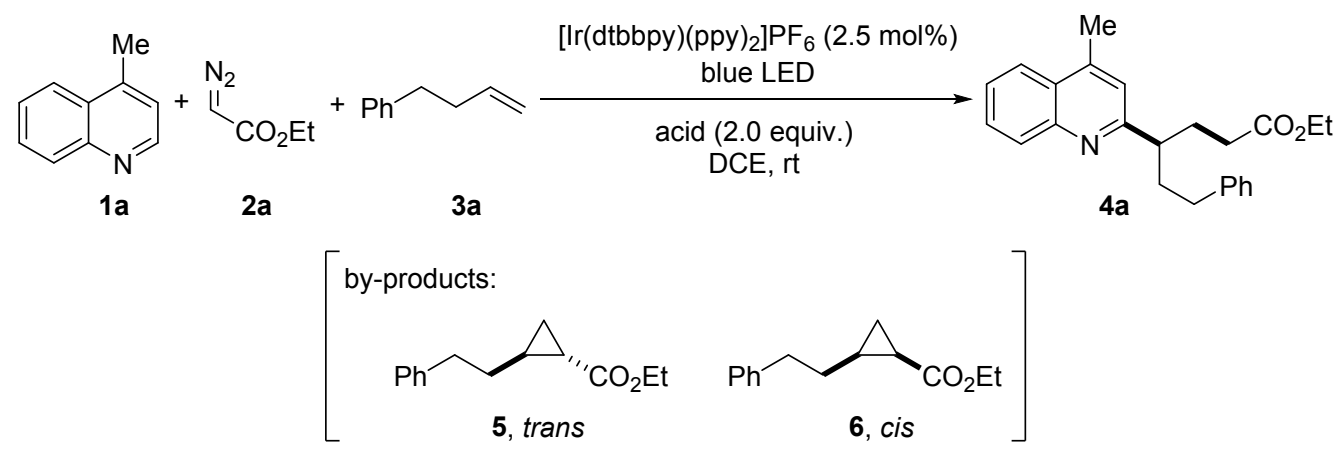

\begin{tabular}{ccc:ccc} 
entry & acid & $\mathbf{4 a / 5 / 6}(\%)^{b}$ & entry & acid & $\mathbf{4 a / 5 / 6}(\mathbf{\%})^{b}$ \\
\hline 1 & $\mathrm{AcOH}$ & $7 / 11 / 11$ & 3 & $\mathrm{CCl}_{3} \mathrm{COOH}$ & $33 / 6 / 6$ \\
2 & & & & & \\
& & & & & \\
$\mathrm{CH}_{3} \mathrm{CH}_{2} \mathrm{COOH}$ & $5 / 13 / 13$ & 4 & &
\end{tabular}

${ }^{a}$ Conditions: 1a $(0.2 \mathrm{mmol}, 1.0$ equiv. $),\left[\operatorname{Ir}(\mathrm{dtbbpy})(\mathrm{ppy})_{2}\right] \mathrm{PF}_{6}(2.5 \mathrm{~mol} \%), 3 \mathrm{a}(0.4 \mathrm{mmol}, 2.0$ equiv. $), 2 \mathrm{a}$ (0.4 mmol, 2.0 equiv.), acid ( $0.4 \mathrm{mmol}, 2.0$ equiv.), DCE $(2.0+1.0 \mathrm{~mL})$, rt, blue LED (Kessil PR160L- 
$467 \mathrm{~nm}, 50 \%$ intensity), $18 \mathrm{~h}$. The solution of $\mathbf{2 a}$ in DCE $(1.0 \mathrm{~mL})$ was added to the reaction mixture via a syringe pump over 5 h. ${ }^{b}$ The yields of $\mathbf{4 a} / \mathbf{5} / \mathbf{6}$ were determined by ${ }^{1} \mathrm{H}$ NMR spectroscopic analyses of the reaction mixture using $\mathrm{CHBr}_{3}$ as the internal standard.

Table S3. Investigation of the feed ratio for the multicomponent reaction. ${ }^{a}$

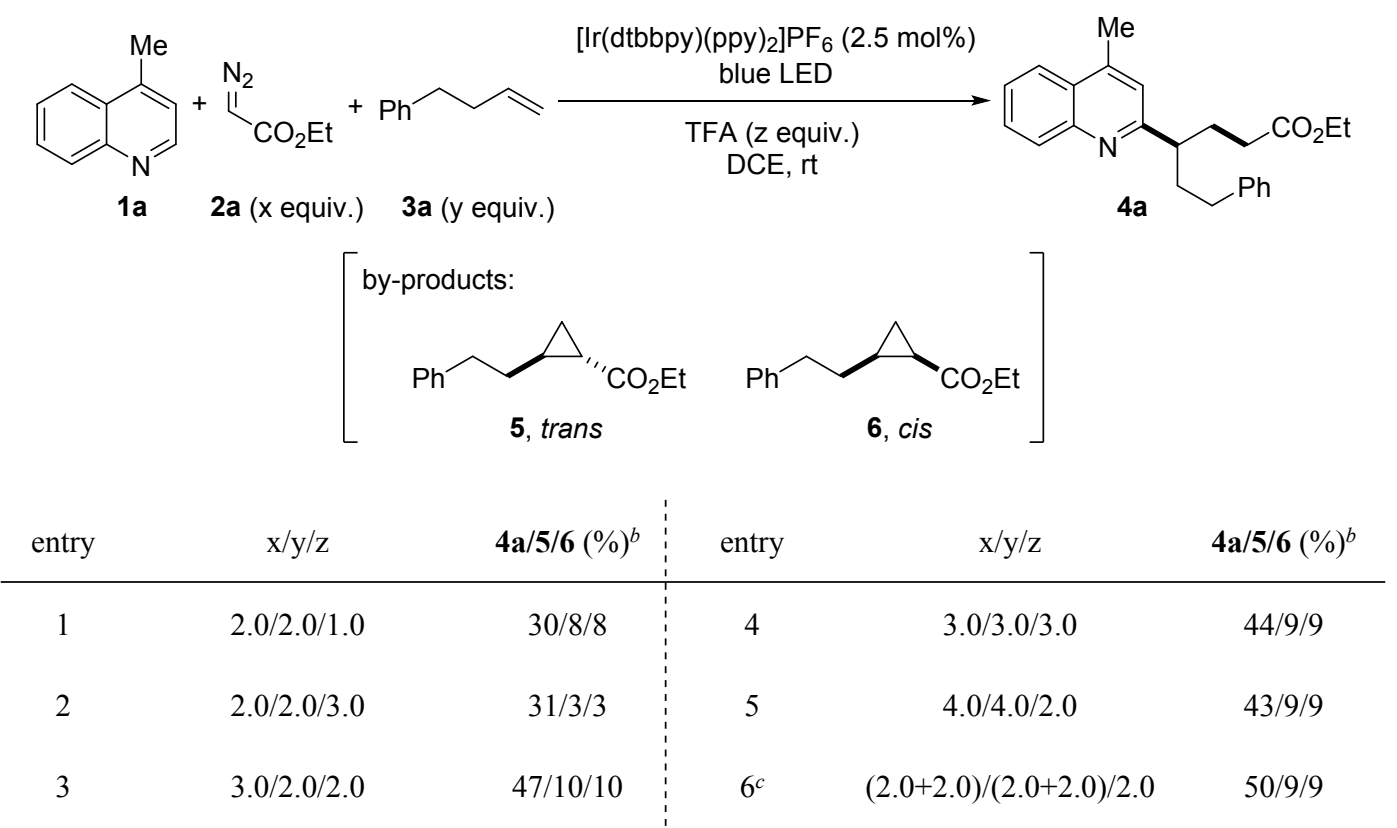

${ }^{a}$ Conditions: 1a ( 0.2 mmol, 1.0 equiv.), [Ir(dtbbpy)(ppy) $\left.)_{2}\right] \mathrm{PF}_{6}(2.5 \mathrm{~mol} \%)$, 3a (y equiv.), 2a (x equiv.), TFA (z equiv.), DCE $(2.0+1.0 \mathrm{~mL}$ ), rt, blue LED (Kessil PR160L-467 nm, 50\% intensity), $18 \mathrm{~h}$. The solution of 2a in DCE $(1.0 \mathrm{~mL})$ was added to the reaction mixture via a syringe pump over $5 \mathrm{~h} .{ }^{b}$ The yields of $\mathbf{4 a} / \mathbf{5} / \mathbf{6}$ were determined by ${ }^{1} \mathrm{H}$ NMR spectroscopic analyses of the reaction mixture using $\mathrm{CHBr}_{3}$ as the internal standard. ${ }^{c} \mathbf{3 a}(0.4 \mathrm{mmol}, 2.0$ equiv. $)$ was added at the beginning of the reaction. The solution of $\mathbf{2 a}(0.4 \mathrm{mmol}, 2.0$ equiv.) in DCE $(1.0 \mathrm{~mL})$ was added to the reaction mixture via a syringe pump over $5 \mathrm{~h}$. After overnight reaction, another dose of $\mathbf{3 a}(0.4 \mathrm{mmol}, 2.0$ equiv.) was added, following by the addition of another dose of $\mathbf{2 a}(0.4 \mathrm{mmol}, 2.0$ equiv. $)$ in DCE $(1.0 \mathrm{~mL})$ via a syringe pump over 5 $\mathrm{h}$.

Table S4. Investigation of the catalytic amount of photocatalyst Ir and the feed ratio for the multicomponent reaction. ${ }^{a}$

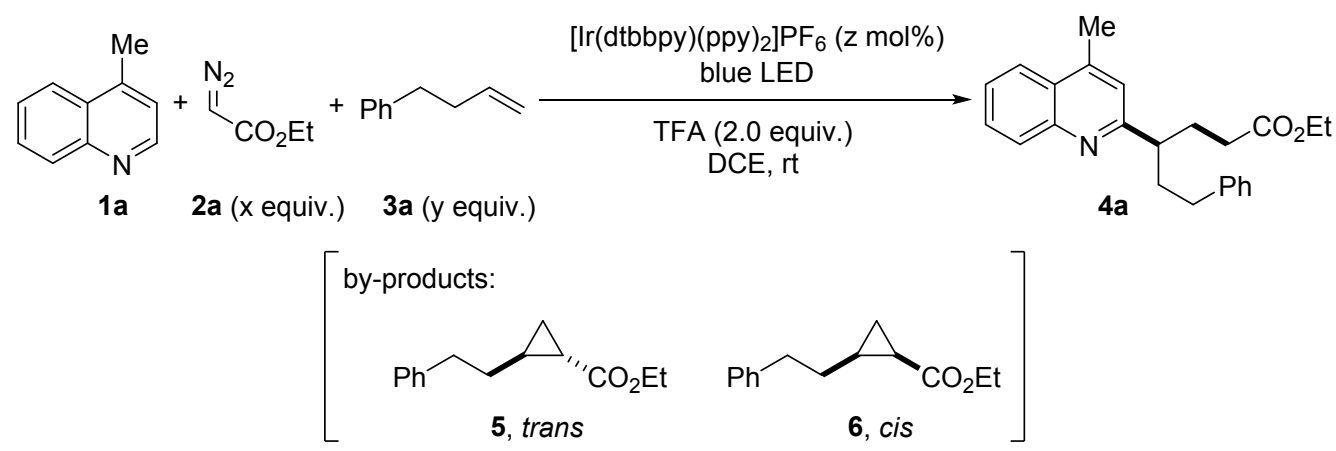




\begin{tabular}{ccc:ccc}
\hline entry & $\mathbf{x} / \mathrm{y} / \mathrm{z}$ & $\mathbf{4 a / 5 / 6}(\%)^{b}$ & entry & $\mathrm{x} / \mathrm{y} / \mathrm{z}$ & $\mathbf{4 a / 5 / 6}(\%)^{b}$ \\
\hline 1 & $(2.0+2.0) /(2.0+2.0) /(1.0+1.0)$ & $61 / 10 / 10$ & $3^{c}$ & $(2.0+2.0) /(2.0+2.0) /(1.5+1.5)$ & $71 / 9 / 9$ \\
$2^{c}$ & $(2.0+2.0) /(2.0+2.0) /(1.0+1.0)$ & $75(73)^{d} / 10 / 10$ & $4^{e}$ & $(2.0+2.0) /(2.0+2.0) /(1.0+1.0)$ & $70 / 9 / 9$ \\
\hline
\end{tabular}

${ }^{a}$ Conditions: 1a (0.2 mmol, 1.0 equiv.), [ $\left.\operatorname{Ir}(\mathrm{dtbbpy})(\mathrm{ppy})_{2}\right] \mathrm{PF}_{6}(\mathrm{z}$ mol\%), 3a (y equiv.), 2a (x equiv.), TFA (0.4 mmol, 2.0 equiv.), DCE $(2.0+1.0+1.0 \mathrm{~mL})$, rt, blue LED (Kessil PR160L-467 nm, 50\% intensity). The solution of $\mathbf{2 a}$ in DCE $(1.0 \mathrm{~mL})$ was added to the reaction mixture via a syringe pump over $5 \mathrm{~h} .{ }^{b}$ The yields of $\mathbf{4 a / 5} / \mathbf{6}$ were determined by ${ }^{1} \mathrm{H}$ NMR spectroscopic analyses of the reaction mixture using $\mathrm{CHBr}_{3}$ as the internal standard. ${ }^{c} \mathbf{3 a}(0.4 \mathrm{mmol}, 2.0$ equiv. $)$ and $\left[\operatorname{Ir}(\mathrm{dtbbpy})(\mathrm{ppy})_{2}\right] \mathrm{PF}_{6}(\mathrm{z} / 2 \mathrm{~mol} \%)$ were added at the beginning of the reaction. The solution of $2 \mathbf{a}(0.4 \mathrm{mmol}, 2.0$ equiv. $)$ in DCE $(1.0 \mathrm{~mL})$ was added to the reaction mixture via a syringe pump over $10 \mathrm{~h}$. After $2 \mathrm{~h}$, another dose of $\left[\operatorname{Ir}(\mathrm{dtbbpy})(\mathrm{ppy})_{2}\right] \mathrm{PF}_{6}(\mathrm{z} / 2$ mol\%) and 3a ( $0.4 \mathrm{mmol}, 2.0$ equiv.) was added, following by the addition of another dose of $2 \mathbf{a}(0.4$ mmol, 2.0 equiv.) in DCE (1.0 mL) via a syringe pump over $10 \mathrm{~h} .{ }^{d}$ Isolated yield in parentheses. ${ }^{e}$ The solution of $2 \mathbf{a}$ in DCE $(1.0 \mathrm{~mL})$ was added to the reaction mixture via a syringe pump over $20 \mathrm{~h}$,

Table S5. Investigation of the suitability of our previous catalytic system for this multicomponent reaction. ${ }^{4}$

(1) Photocatalytic system:

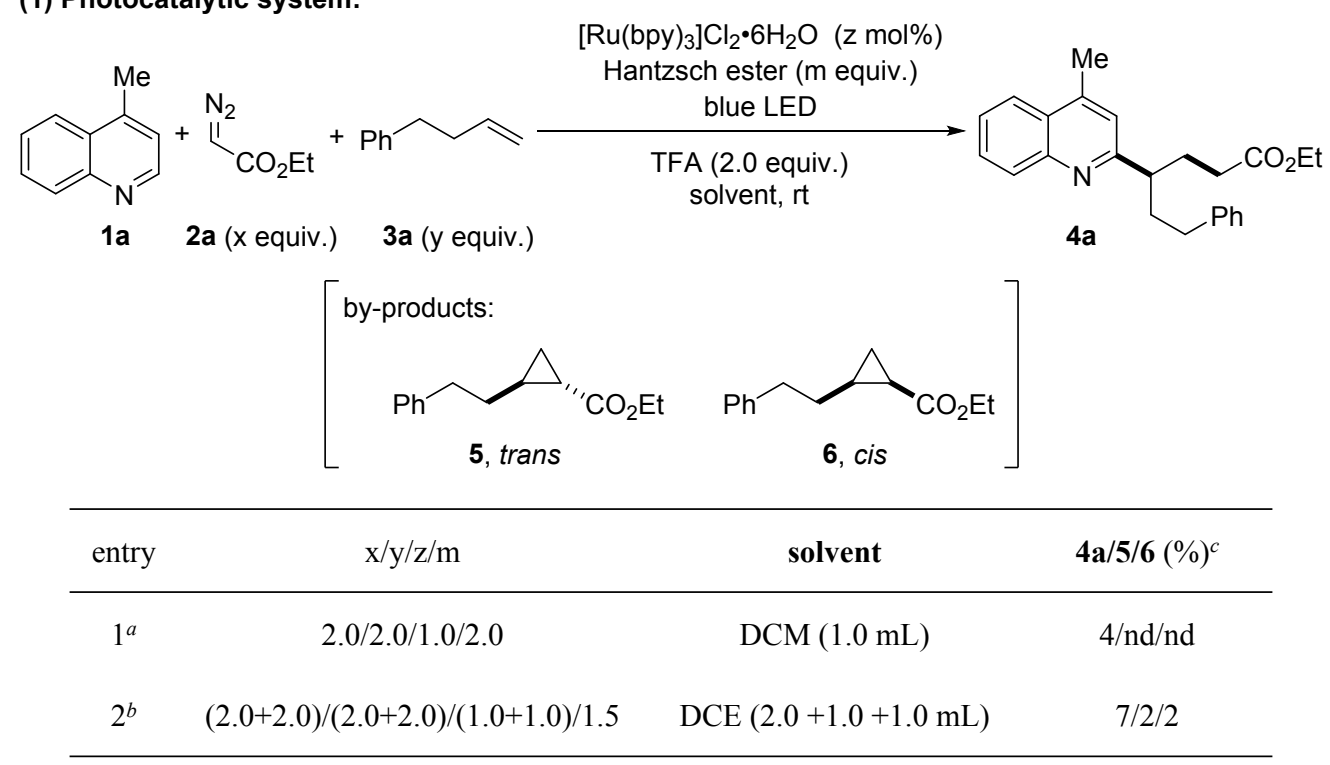

${ }^{a}$ Conditions: $1 \mathbf{a}(0.2 \mathrm{mmol}, 1.0$ equiv. $\left.), \mathrm{Ru}(\mathrm{bpy})_{3}\right] \mathrm{Cl}_{2} \bullet 6 \mathrm{H}_{2} \mathrm{O}(1.0 \mathrm{~mol} \%), 3 \mathbf{3 a}(4.0$ equiv.), $2 \mathrm{a}(4.0$ equiv.), TFA (0.4 mmol, 2.0 equiv.), Hantzsch ester ( 0.4 mmol, 2.0 equiv.), DCM (1.0 mL), rt, blue LED (Kessil PR160L-467 nm, 50\% intensity), 18 h. ${ }^{b}$ Conditions: 1 a ( $0.2 \mathrm{mmol}, 1.0$ equiv.), $\left.\mathrm{Ru}(\mathrm{bpy})_{3}\right] \mathrm{Cl}_{2} \cdot 6 \mathrm{H}_{2} \mathrm{O}(2.0$ mol\%), 3 a (4.0 equiv.), 2 a (4.0 equiv.), TFA (0.4 mmol, 2.0 equiv.), Hantzsch ester (0.3 mmol, 1.5 equiv.), DCE $(2.0+1.0+1.0 \mathrm{~mL})$, rt, blue LED (Kessil PR160L-467 nm, 50\% intensity). 3a (0.4 mmol, 2.0 equiv.) and $\left.\mathrm{Ru}(\mathrm{bpy})_{3}\right] \mathrm{Cl}_{2} \cdot 6 \mathrm{H}_{2} \mathrm{O}(1.0 \mathrm{~mol} \%)$ were added at the beginning of the reaction. The solution of $2 \mathrm{a}(0.4 \mathrm{mmol}, 2.0$ equiv. $)$ in DCE $(1.0 \mathrm{~mL})$ was added to the reaction mixture via a syringe pump over $10 \mathrm{~h}$. After $2 \mathrm{~h}$, another dose of $\left.\mathrm{Ru}(\mathrm{bpy})_{3}\right] \mathrm{Cl}_{2} \cdot 6 \mathrm{H}_{2} \mathrm{O}(1.0 \mathrm{~mol} \%)$ and $\mathbf{3 a}(0.4 \mathrm{mmol}, 2.0$ equiv. $)$ was added, following by the addition of another dose of $\mathbf{2 a}(0.4 \mathrm{mmol}, 2.0$ equiv. $)$ in DCE $(1.0 \mathrm{~mL})$ via a syringe pump over $10 \mathrm{~h}$. ${ }^{c}$ The yields of $\mathbf{4 a / 5 / 6}$ were determined by ${ }^{1} \mathrm{H}$ NMR spectroscopic analyses of the reaction mixture using $\mathrm{CHBr}_{3}$ as the internal standard. nd $=$ not detected. 
(2) Iron-catalytic system: ${ }^{\alpha}$
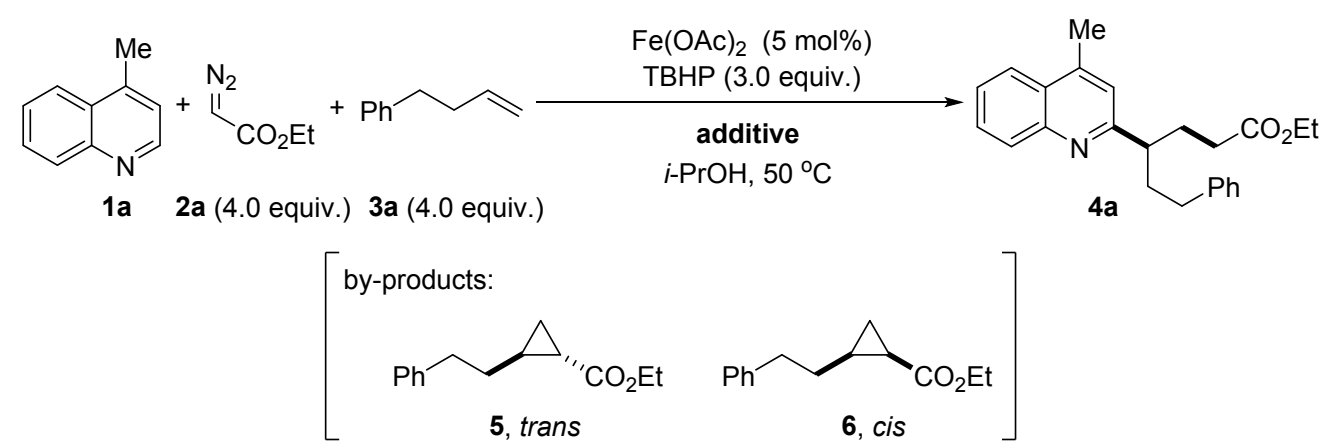

\begin{tabular}{ccc} 
entry & additive & $\mathbf{4 a / 5 / 6}(\%)^{b}$ \\
\hline 1 & TFA (2.0 equiv.) & $\mathrm{nd} / \mathrm{nd} / \mathrm{nd}$ \\
2 & TFA (2 equiv. $)+\mathrm{Et}_{3} \mathrm{~N}(2$ equiv. $)$ & $\mathrm{nd} / \mathrm{nd} / \mathrm{nd}$ \\
3 & $\mathrm{Et}_{3} \mathrm{~N}(2.0$ equiv. $)$ & $\mathrm{nd} / \mathrm{nd} / \mathrm{nd}$
\end{tabular}

${ }^{a}$ Conditions: 1a ( $0.2 \mathrm{mmol}, 1.0$ equiv.), $\mathrm{Fe}(\mathrm{OAc})_{2}(5 \mathrm{~mol} \%)$, 2a ( $0.8 \mathrm{mmol}, 4.0$ equiv.), 3a ( $0.8 \mathrm{mmol}$, 4.0 equiv.), additive, $i-\mathrm{PrOH}(3.0 \mathrm{~mL}), 50^{\circ} \mathrm{C}, 18 \mathrm{~h}, \mathrm{~N}_{2}$ atmosphere. ${ }^{b}$ The yields of $\mathbf{4 a} / \mathbf{5} / \mathbf{6}$ were determined by ${ }^{1} \mathrm{H}$ NMR spectroscopic analyses of the reaction mixture using $\mathrm{CHBr}_{3}$ as the internal standard. $\mathrm{nd}=$ not detected.

\section{General Procedure and Characterization of Products}

To a reaction vial equipped with a magnetic stir bar were added $\operatorname{Ir}(\mathrm{dtbbpy})(\mathrm{ppy})_{2} \mathrm{PF}_{6}$ (1.8 mg, $0.002 \mathrm{mmol}, 1 \mathrm{~mol} \%)$, anhydrous DCE $(2.0 \mathrm{~mL})$, heteroarene $1(0.2 \mathrm{mmol}$, 1.0 equiv.), alkene 3 ( $0.4 \mathrm{mmol}, 2.0$ equiv.) and TFA (45.6 mg, $0.4 \mathrm{mmol}, 2.0$ equiv.). The mixture was degassed for 6 minutes by bubbling $\mathrm{N}_{2}$ through the solution. The vial was then placed on a stir plate directly in front of two blue LED lamps (Kessil PR160L$467 \mathrm{~nm}, 50 \%$ intensity) approximately $8.5 \mathrm{~cm}$ away. The solution of diazo compound 2 (0.4 mmol, 2.0 equiv.) in DCE (1.0 mL) was slowly added into the reaction mixture via a syringe pump over $10 \mathrm{~h}$ under a $\mathrm{N}_{2}$ atmosphere. After another $2 \mathrm{~h}$, $\operatorname{Ir}(\mathrm{dtbbpy})(\mathrm{ppy})_{2} \mathrm{PF}_{6}(1.8 \mathrm{mg}, 0.002 \mathrm{mmol}, 1 \mathrm{~mol} \%$ ) and alkene 3 ( $0.4 \mathrm{mmol}, 2.0$ equiv.) were added, and then the reaction mixture was degassed for 6 minutes by bubbling $\mathrm{N}_{2}$. Another dose of diazo compound 2 ( $0.4 \mathrm{mmol}, 2.0$ equiv. $)$ in DCE (1.0 mL) was slowly added into the reaction mixture via a syringe pump over $10 \mathrm{~h}$. The resulting solution was irradiated for another $2-8 \mathrm{~h}$. The reaction temperature was approximately $25^{\circ} \mathrm{C}$ with two fans used to assist cooling. The reaction was quenched by adding saturated aqueous $\mathrm{Na}_{2} \mathrm{CO}_{3}(4.0 \mathrm{~mL}), \mathrm{H}_{2} \mathrm{O}(15.0 \mathrm{~mL})$, extracted with DCM $(15.0 \mathrm{~mL} \times 3)$, and the combined organic layers were dried over anhydrous $\mathrm{Na}_{2} \mathrm{SO}_{4}$, and then condensed under reduced pressure. The crude mixture was then purified by column chromatography on silica gel with hexanes/ethyl acetate mixtures as eluent to give the corresponding products. See below picture for details: 

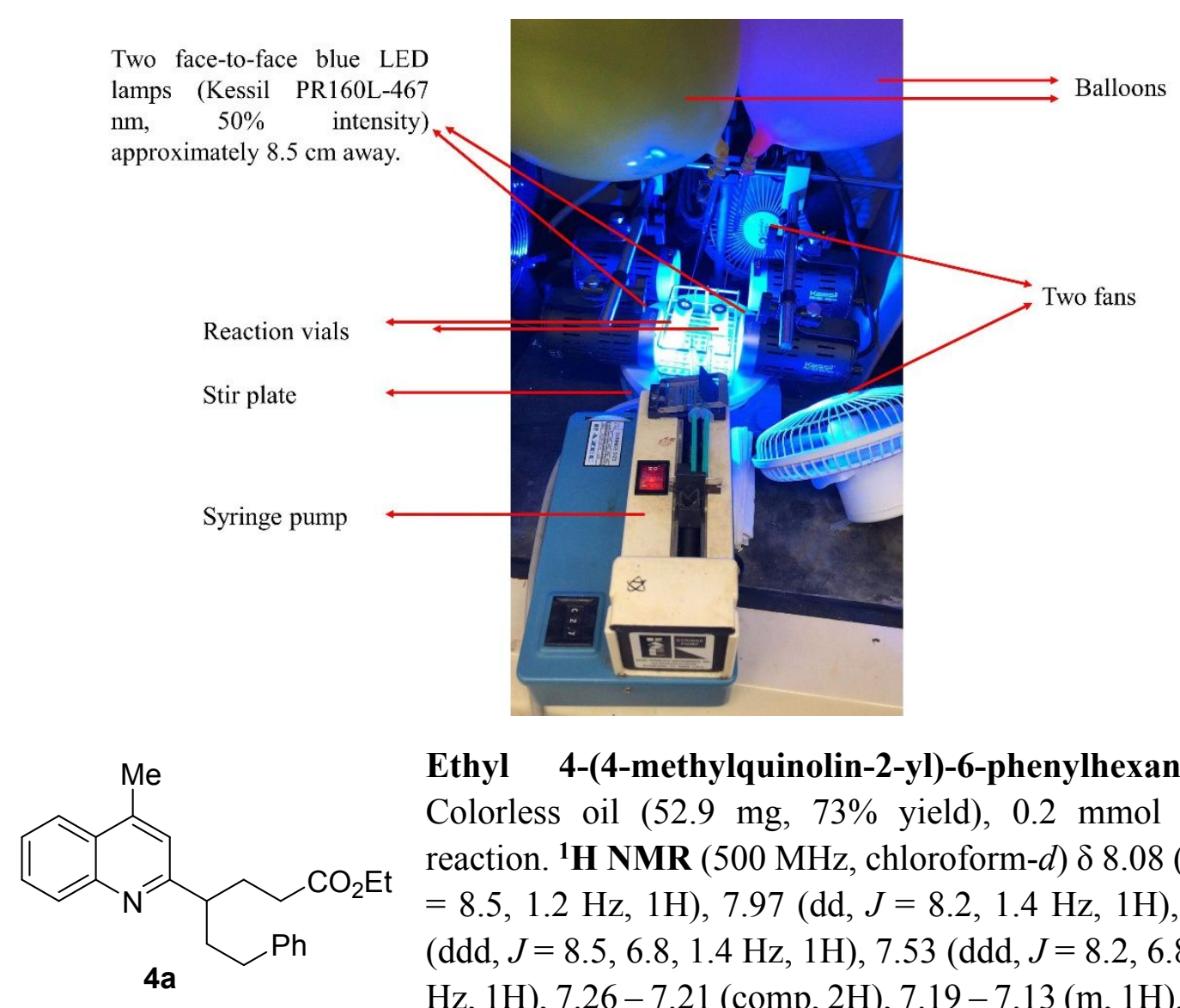

Ethyl 4-(4-methylquinolin-2-yl)-6-phenylhexanoate. Colorless oil (52.9 mg, 73\% yield), $0.2 \mathrm{mmol}$ scale reaction. ${ }^{1} \mathbf{H}$ NMR (500 MHz, chloroform- $d$ ) $\delta 8.08(\mathrm{dd}, J$ $=8.5,1.2 \mathrm{~Hz}, 1 \mathrm{H}), 7.97(\mathrm{dd}, J=8.2,1.4 \mathrm{~Hz}, 1 \mathrm{H}), 7.69$ (ddd, $J=8.5,6.8,1.4 \mathrm{~Hz}, 1 \mathrm{H}), 7.53(\mathrm{ddd}, J=8.2,6.8,1.2$ $\mathrm{Hz}, 1 \mathrm{H}), 7.26-7.21$ (comp, 2H), $7.19-7.13$ (m, 1H), 7.15 - 7.09 (comp, 3H), 4.08 - 4.02 (comp, 2H), $3.01-2.95$ (m, 1H), 2.70 (s, 3H), 2.63 $2.54(\mathrm{~m}, 1 \mathrm{H}), 2.52-2.46(\mathrm{~m}, 1 \mathrm{H}), 2.28-2.11$ (comp, 5H), $2.11-2.02(\mathrm{~m}, 1 \mathrm{H}), 1.19$ (t, $J=7.1 \mathrm{~Hz}, 3 \mathrm{H}) .{ }^{13} \mathbf{C}$ NMR $(126 \mathrm{MHz}$, chloroform- $d$ ) $\delta 173.7,164.0,147.9,144.5$, $142.4,129.8,129.1,128.5,128.4,127.3,125.8,125.8,123.7,121.2,60.3,47.7,37.3$, 33.9, 32.5, 30.6, 19.0, 14.3. HRMS (ESI) scaled for $[\mathrm{M}+\mathrm{H}]^{+}: \mathrm{C}_{24} \mathrm{H}_{28} \mathrm{NO}_{2}, \mathrm{~m} / \mathrm{z}$ : 362.2115, observed: 362.2113 .<smiles>CCCCC(CCC(C)=O)c1cc(C)c2ccccc2n1</smiles>

Ethyl 4-(4-methylquinolin-2-yl)octanoate. Colorless oil (46.0 $\mathrm{mg}, 73 \%$ yield), $0.2 \mathrm{mmol}$ scale reaction. ${ }^{1} \mathbf{H}$ NMR $(500 \mathrm{MHz}$, chloroform- $d) \delta 8.04(\mathrm{dd}, J=8.3,1.2 \mathrm{~Hz}, 1 \mathrm{H})$, $7.95(\mathrm{dd}, J=8.2,1.2 \mathrm{~Hz}, 1 \mathrm{H}), 7.67$ (ddd, $J=8.3,6.9,1.2$ $\mathrm{Hz}, 1 \mathrm{H}), 7.50$ (ddd, $J=8.2,6.9,1.2 \mathrm{~Hz}, 1 \mathrm{H}), 7.10$ (s, 1H), $4.09-4.00$ (comp, 2H), $2.94-2.84(\mathrm{~m}, 1 \mathrm{H}), 2.68(\mathrm{~s}, 3 \mathrm{H})$, $2.29-2.19$ (m, 1H), $2.17-2.07$ (comp, 3H), $1.83-1.69$ (comp, 3H), $1.33-1.25$ (comp, 3H), 1.18 (t, $J=7.1 \mathrm{~Hz}, 3 \mathrm{H}), 0.82(\mathrm{t}, J=7.1 \mathrm{~Hz}, 3 \mathrm{H}) .{ }^{13} \mathbf{C}$ NMR $(126 \mathrm{MHz}$, chloroform- $d$ ) $\delta 173.8,164.7,147.9,144.3,129.8,129.0,127.2,125.6,123.7,121.0$, 60.3, 48.2, 35.5, 32.6, 30.6, 29.9, 22.9, 19.0, 14.3, 14.1. HRMS (ESI) scaled for $[\mathrm{M}+\mathrm{H}]^{+}: \mathrm{C}_{20} \mathrm{H}_{28} \mathrm{NO}_{2}, \mathrm{~m} / \mathrm{z}: 314.2115$, observed: 314.2115 .<smiles>CCCCCCC(CCC(=O)OCC)c1cc(C)c2ccccc2n1</smiles>

Ethyl 4-(4-methylquinolin-2-yl)decanoate. Colorless oil (51.7 mg, 75\% yield), $0.2 \mathrm{mmol}$ scale reaction. ${ }^{\mathbf{1}} \mathbf{H}$ NMR (500 MHz, chloroform- $d$ ) $\delta 8.04$ (dd, $J=8.4,1.2$ $\mathrm{Hz}, 1 \mathrm{H}), 7.94(\mathrm{dd}, J=8.2,1.4 \mathrm{~Hz}, 1 \mathrm{H}), 7.66(\mathrm{ddd}, J=$ $8.4,6.8,1.4 \mathrm{~Hz}, 1 \mathrm{H}), 7.50(\mathrm{ddd}, J=8.2,6.8,1.2 \mathrm{~Hz}, 1 \mathrm{H})$, 
7.09 (s, 1H), $4.07-4.01$ (comp, 2H), $2.93-2.85(\mathrm{~m}, 1 \mathrm{H}), 2.68(\mathrm{~s}, 3 \mathrm{H}), 2.28-2.19(\mathrm{~m}$, 1H), $2.17-2.05$ (comp, 3H), $1.85-1.76(\mathrm{~m}, 1 \mathrm{H}), 1.75-1.67(\mathrm{~m}, 1 \mathrm{H}), 1.33-1.20$ (comp, 8H), 1.18 (t, $J=7.1 \mathrm{~Hz}, 3 \mathrm{H}), 0.82$ (t, $J=7.0 \mathrm{~Hz}, 3 \mathrm{H}) .{ }^{13} \mathbf{C}$ NMR (126 MHz, chloroform- $d$ ) $\delta 173.8,164.7,147.8,144.3,129.8,129.0,127.2,125.6,123.7,121.0$, 60.3, 48.2 35.8, 32.6, 31.8, 30.6, 29.5, 27.7, 22.7, 19.0, 14.3, 14.2. HRMS (ESI) scaled for $[\mathrm{M}+\mathrm{H}]^{+}: \mathrm{C}_{22} \mathrm{H}_{32} \mathrm{NO}_{2}, \mathrm{~m} / \mathrm{z}: 342.2428$, observed: 342.2428.<smiles>CCOC(=O)CCC(Cc1ccccc1)c1cc(C)c2ccccc2n1</smiles>
(s, 3H), $2.22-2.08$ (comp, 4H), 1.16 (t, $J=7.1 \mathrm{~Hz}, 3 \mathrm{H}) .{ }^{13} \mathbf{C}$ NMR (126 MHz, chloroform- $d$ ) $\delta 173.6,163.6,147.9,144.3,140.3,129.8,129.3,129.1,128.3,127.2$, 126.1, 125.7, 123.7, 121.9, 60.3, 49.6, 41.9, 32.5, 29.6, 18.9, 14.3. HRMS (ESI) scaled for $[\mathrm{M}+\mathrm{H}]^{+}: \mathrm{C}_{23} \mathrm{H}_{26} \mathrm{NO}_{2}, \mathrm{~m} / \mathrm{z}$ : 348.1958, observed: 348.1958.<smiles>CCOC(=O)CCC(C)c1cc(C)c2ccccc2n1</smiles>

Ethyl 4-(4-methylquinolin-2-yl)-5-phenylpentanoate. Colorless oil (39.0 mg, 56\% yield), $0.2 \mathrm{mmol}$ scale reaction. ${ }^{1} \mathbf{H}$ NMR (500 MHz, chloroform- $d$ ) $\delta 8.08$ (dd, $J$ $=8.5,1.2 \mathrm{~Hz}, 1 \mathrm{H}), 7.94(\mathrm{dd}, J=8.2,1.4 \mathrm{~Hz}, 1 \mathrm{H}), 7.68$ (ddd, $J=8.5,6.8,1.4 \mathrm{~Hz}, 1 \mathrm{H}), 7.51$ (ddd, $J=8.2,6.8,1.2$ $\mathrm{Hz}, 1 \mathrm{H}$ ), $7.23-7.16$ (comp, 2H), $7.17-7.10$ (comp, 3H),

Ethyl 4-cyclohexyl-4-(4-methylquinolin-2-yl)butanoate. Colorless oil ( $49.1 \mathrm{mg}, 72 \%$ yield), $0.2 \mathrm{mmol} \mathrm{scale}$ reaction. ${ }^{1} \mathbf{H}$ NMR $(500 \mathrm{MHz}$, chloroform- $d$ ) $\delta 8.04$ (dd, $J$ $=8.4,1.2 \mathrm{~Hz}, 1 \mathrm{H}), 7.94(\mathrm{dd}, J=8.3,1.4 \mathrm{~Hz}, 1 \mathrm{H}), 7.66$ (ddd, $J=8.4,6.8,1.4 \mathrm{~Hz}, 1 \mathrm{H}$ ), 7.49 (ddd, $J=8.3,6.8,1.2$ $\mathrm{Hz}, 1 \mathrm{H}), 7.06$ (s, 1H), $4.06-3.99$ (comp, 2H), $2.68-2.65$ (comp, 4H), $2.30-2.21$ (m, 1H), 2.16- 2.06 (comp, 2H), $2.07-1.97$ (comp, 2H), 1.76 - 1.68 (comp, 2H), 1.62 - 1.54 (comp, 2H), $1.34-1.20$ (comp, 3H), 1.16 (t, J=7.2 Hz, 3H), 1.13 - 1.01 (comp, 3H). ${ }^{13}$ C NMR (126 MHz, chloroform-d) $\delta 173.9,164.1,147.8$, 143.9, 129.8, 129.0, 127.2, 125.6, 123.7, 121.9, 60.2, 54.3, 42.6, 32.8, 31.7, 31.1, 27.0, 26.64, 26.58, 26.5, 19.0, 14.3. HRMS (ESI) scaled for $[\mathrm{M}+\mathrm{H}]^{+}: \mathrm{C}_{22} \mathrm{H}_{30} \mathrm{NO}_{2}, \mathrm{~m} / \mathrm{z}$ : 340.2271, observed: 340.2272 .<smiles>CCOC(=O)CCC(COC)c1cc(C)c2ccccc2n1</smiles>

Ethyl 5-methoxy-4-(4-methylquinolin-2-yl)pentanoate. Colorless oil (46.2 mg, 77\% yield), $0.2 \mathrm{mmol}$ scale reaction. ${ }^{1} \mathbf{H}$ NMR $(500 \mathrm{MHz}$, chloroform- $d$ ) $\delta 8.04(\mathrm{dd}, J$ $=8.4,1.2 \mathrm{~Hz}, 1 \mathrm{H}), 7.95(\mathrm{dd}, J=8.3,1.4 \mathrm{~Hz}, 1 \mathrm{H}), 7.67$ (ddd, $J=8.4,6.7,1.4 \mathrm{~Hz}, 1 \mathrm{H}), 7.51$ (ddd, $J=8.3,6.7,1.2$ $\mathrm{Hz}, 1 \mathrm{H}), 7.15$ (s, 1H), $4.08-4.03$ (comp, 2H), 3.79 (dd, $J$ $=9.5,6.7 \mathrm{~Hz}, 1 \mathrm{H}), 3.69(\mathrm{dd}, J=9.5,6.7 \mathrm{~Hz}, 1 \mathrm{H}), 3.32(\mathrm{~s}, 3 \mathrm{H}), 3.26-3.18(\mathrm{~m}, 1 \mathrm{H})$, 2.68 (s, 3H), $2.34-2.20$ (comp, 2H), $2.22-2.13$ (comp, 2H), 1.19 (t, J=7.2 Hz, 3H). ${ }^{13}$ C NMR (126 MHz, chloroform- $d$ ) $\delta$ 173.6, 162.0, 148.0, 144.4, 129.9, 129.1, 127.4, 125.8, 123.7, 122.0, 76.3, 60.4, 59.1, 48.0, 32.3, 27.1, 18.9, 14.3. HRMS (ESI) scaled for $[\mathrm{M}+\mathrm{H}]^{+}: \mathrm{C}_{18} \mathrm{H}_{24} \mathrm{NO}_{3}, \mathrm{~m} / \mathrm{z}: 302.1751$, observed: 302.1751 .<smiles>CCOC(=O)CCC(CCCCl)c1cc(C)c2ccccc2n1</smiles>

Ethyl 8-chloro-4-(4-methylquinolin-2-yl)octanoate. Colorless oil (54.4 mg, 78\% yield), $0.2 \mathrm{mmol}$ scale reaction. ${ }^{1} \mathbf{H}$ NMR $(500 \mathrm{MHz}$, chloroform- $d$ ) $\delta 8.04$ (dd, $J$ $=8.5,1.3 \mathrm{~Hz}, 1 \mathrm{H}), 7.95(\mathrm{dd}, J=8.3,1.3 \mathrm{~Hz}, 1 \mathrm{H}), 7.67$ 
(ddd, $J=8.5,6.8,1.3 \mathrm{~Hz}, 1 \mathrm{H}), 7.51(\mathrm{ddd}, J=8.3,6.8,1.3 \mathrm{~Hz}, 1 \mathrm{H}), 7.09$ (s, 1H), 4.08 - 4.00 (comp, 2H), 3.47 - 3.42 (comp, 2H), $2.95-2.87$ (m, 1H), 2.68 (s, 3H), $2.28-$ 2.19 (m, 1H), $2.16-2.06$ (comp, 2H), $1.90-1.83$ (m, 1H), $1.80-1.66$ (comp, 4H), $1.49-1.39(\mathrm{~m}, 1 \mathrm{H}), 1.32-1.22(\mathrm{~m}, 1 \mathrm{H}), 1.18(\mathrm{t}, J=7.1 \mathrm{~Hz}, 3 \mathrm{H}) .{ }^{13} \mathbf{C}$ NMR $(126 \mathrm{MHz}$, chloroform- $d$ ) $\delta 173.7,164.1,147.9,144.5,129.8,129.1,127.2,125.7,123.7,121.1$, 60.3, 47.9, 44.9, 34.8, 32.8, 32.5, 30.5, 25.0, 19.0, 14.3. HRMS (ESI) scaled for $[\mathrm{M}+\mathrm{H}]^{+}: \mathrm{C}_{20} \mathrm{H}_{27} \mathrm{ClNO}_{2}, \mathrm{~m} / \mathrm{z}: 348.1725$, observed: 348.1726.

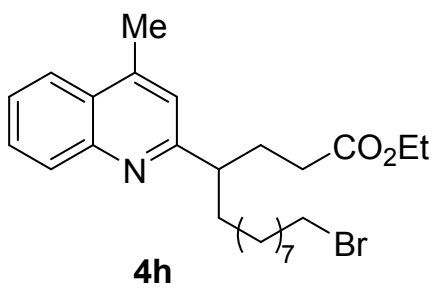

Ethyl 13-bromo-4-(4-methylquinolin-2-yl)tridecanoate. Colorless oil ( $74.5 \mathrm{mg}, 81 \%$ yield), $0.2 \mathrm{mmol}$ scale reaction. ${ }^{1}$ H NMR (500 MHz, chloroform- $d$ ) $\delta 8.04(\mathrm{dd}, J=8.4,1.2$ $\mathrm{Hz}, 1 \mathrm{H}), 7.94(\mathrm{dd}, J=8.2,1.4 \mathrm{~Hz}, 1 \mathrm{H}), 7.66(\mathrm{ddd}, J=8.4$, $6.8,1.4 \mathrm{~Hz}, 1 \mathrm{H}), 7.50$ (ddd, $J=8.2,6.8,1.2 \mathrm{~Hz}, 1 \mathrm{H}), 7.09$ (s, 1H), $4.07-3.99$ (comp, 2H), $3.41-3.37$ (m, 1H), 3.36 $(\mathrm{t}, J=6.9 \mathrm{~Hz}, 2 \mathrm{H}), 2.88(\mathrm{tt}, J=8.7,5.9 \mathrm{~Hz}, 1 \mathrm{H}), 2.68(\mathrm{~s}, 3 \mathrm{H}), 2.27-2.20(\mathrm{~m}, 1 \mathrm{H}), 2.16$ - 2.06 (comp, 3H), 1.85 - 1.76 (comp, 4H), $1.74-1.67$ (m, 1H), 1.39 - 1.32 (comp, $3 \mathrm{H}), 1.28-1.22$ (comp, 7H), 1.17 (t, $J=7.1 \mathrm{~Hz}, 3 \mathrm{H}) .{ }^{13} \mathbf{C}$ NMR (126 MHz, chloroformd) $\delta 173.8,164.6,147.8,144.4,129.8,129.0,127.2,125.6,123.7,121.0,60.3,48.2$, 35.7, 34.1, 32.9, 32.6, 30.6, 29.7, 29.4, 28.8, 28.2, 27.6, 19.0, 14.3. Peak overlapping was observed. HRMS (ESI) scaled for $[\mathrm{M}+\mathrm{H}]^{+}: \mathrm{C}_{25} \mathrm{H}_{37} \mathrm{BrNO}_{2}, \mathrm{~m} / \mathrm{z}: 462.2002$, observed: 462.2002 .

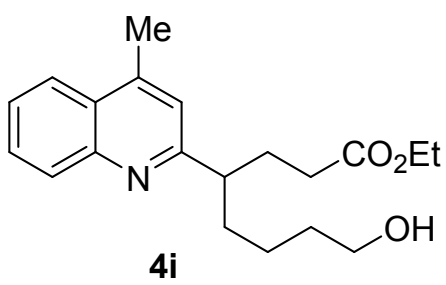

Ethyl 8-hydroxy-4-(4-methylquinolin-2-yl)octanoate. Colorless oil (46.1 mg, 70\% yield), $0.2 \mathrm{mmol}$ scale reaction. ${ }^{1} \mathbf{H}$ NMR $(500 \mathrm{MHz}$, chloroform- $d$ ) $\delta 8.04$ (dd, $J$ $=8.5,1.1 \mathrm{~Hz}, 1 \mathrm{H}), 7.94(\mathrm{dd}, J=8.3,1.4 \mathrm{~Hz}, 1 \mathrm{H}), 7.66$ (ddd, $J=8.5,6.8,1.4 \mathrm{~Hz}, 1 \mathrm{H}), 7.50(\mathrm{ddd}, J=8.3,6.8,1.1$ $\mathrm{Hz}, 1 \mathrm{H}), 7.10$ (s, 1H), $4.07-4.01$ (comp, 2H), 3.57 (t, $J=$ $6.5 \mathrm{~Hz}, 2 \mathrm{H}), 2.95-2.89(\mathrm{~m}, 1 \mathrm{H}), 2.68(\mathrm{~s}, 3 \mathrm{H}), 2.29-2.19(\mathrm{~m}, 1 \mathrm{H}), 2.18-2.11(\mathrm{~m}$, 1H), $2.13-2.04$ (comp, 2H), $1.93-1.82(\mathrm{~m}, 1 \mathrm{H}), 1.79-1.70(\mathrm{~m}, 1 \mathrm{H}), 1.59-1.48$ (comp, 2H), $1.38-1.30$ (comp, 2H), 1.18 (t, $J=7.1 \mathrm{~Hz}, 3 \mathrm{H}) .{ }^{13} \mathbf{C}$ NMR $(126 \mathrm{MHz}$, chloroform- $d$ ) $\delta 173.7,164.3,147.7,144.7,129.6,129.2,127.2,125.8,123.7,121.1$, 62.7, 60.4, 47.9, 35.1, 32.7, 32.5, 30.7, 23.8, 19.0, 14.3. HRMS (ESI) scaled for $[\mathrm{M}+\mathrm{H}]^{+}: \mathrm{C}_{20} \mathrm{H}_{28} \mathrm{NO}_{3}, \mathrm{~m} / \mathrm{z}: 330.2064$, observed: 330.2066 .<smiles>CCCCC(CCC(=O)OCC)c1cc(C)c2ccccc2n1</smiles>

Ethyl 13-hydroxy-4-(4-methylquinolin-2-yl)tridecanoate. White solid (67.9 $\mathrm{mg}, 85 \%$ yield), $0.2 \mathrm{mmol}$ scale reaction. m.p. 58.1-59.1 ${ }^{\circ} \mathrm{C}$. ${ }^{1} \mathbf{H}$ NMR $(500 \mathrm{MHz}$, chloroform- $d$ ) $\delta 8.04$ (dd, $J=8.4,1.2 \mathrm{~Hz}, 1 \mathrm{H}), 7.94$ (dd, $J$ $=8.2,1.3 \mathrm{~Hz}, 1 \mathrm{H}), 7.65(\mathrm{ddd}, J=8.4,6.9,1.3 \mathrm{~Hz}, 1 \mathrm{H})$, 7.49 (ddd, $J=8.2,6.9,1.2 \mathrm{~Hz}, 1 \mathrm{H}), 7.09$ (s, 1H), $4.06-$ 4.00 (comp, 2H), $3.66-3.56$ (comp, 2H), $2.94-2.83$ (m, 1H), 2.67 (s, 3H), $2.29-2.18$ (m, 1H), $2.15-2.03$ (comp, 3H), $1.82-1.74(\mathrm{~m}, 1 \mathrm{H}), 1.72-1.65(\mathrm{~m}, 1 \mathrm{H}), 1.54-1.46$ (comp, 2H), $1.34-1.19$ (comp, 12H), 1.17 (t, $J=7.1 \mathrm{~Hz}, 3 \mathrm{H}) .{ }^{13} \mathbf{C}$ NMR (126 MHz, chloroform- $d$ ) $\delta 173.8,164.7,147.8,144.4,129.7,129.0,127.2,125.6,123.7,120.9$, 63.0, 60.3, 48.2, 35.7, 32.9, 32.6, 30.6, 29.7, 29.5, 29.4, 27.6, 25.8, 19.0, 14.3. Peak 
overlapping was observed. HRMS (ESI) scaled for $[\mathrm{M}+\mathrm{H}]^{+}: \mathrm{C}_{25} \mathrm{H}_{38} \mathrm{NO}_{3}, \mathrm{~m} / \mathrm{z}$ : 400.2846, observed: 400.2850 .<smiles>CCOC(=O)CCC(CCC(=O)OCC)c1cc(C)c2ccccc2n1</smiles>

Diethyl 4-(4-methylquinolin-2-yl)heptanedioate. Colorless oil (51.2 $\mathrm{mg}, 72 \%$ yield), $0.2 \mathrm{mmol}$ scale reaction. ${ }^{1} \mathbf{H}$ NMR $(500 \mathrm{MHz}$, chloroform- $d$ ) $\delta 8.02(\mathrm{dd}, J$ $=8.4,1.2 \mathrm{~Hz}, 1 \mathrm{H}), 7.94(\mathrm{dd}, J=8.3,1.4 \mathrm{~Hz}, 1 \mathrm{H}), 7.66$ (ddd, $J=8.4,6.7,1.4 \mathrm{~Hz}, 1 \mathrm{H}), 7.50$ (ddd, $J=8.3,6.7,1.2$ $\mathrm{Hz}, 1 \mathrm{H}), 7.10$ (s, 1H), $4.09-4.01$ (comp, 4H), $2.97-2.89$ (m, 1H), 2.68 (s, 3H), $2.31-2.22$ (comp, 2H), $2.19-2.06$ (comp, 6H), 1.18 (t, $J=7.1$ $\mathrm{Hz}, 6 \mathrm{H}) .{ }^{13} \mathbf{C}$ NMR $(126 \mathrm{MHz}$, chloroform- $d$ ) $\delta 173.5,163.2,147.9,144.7,129.8,129.1$, 127.3, 125.8, 123.7, 121.3, 60.4, 47.3, 32.4, 30.4, 18.9, 14.3. HRMS (ESI) scaled for $[\mathrm{M}+\mathrm{H}]^{+}: \mathrm{C}_{21} \mathrm{H}_{28} \mathrm{NO}_{4}, \mathrm{~m} / \mathrm{z}: 358.2013$, observed: 358.2014.<smiles>CCOC(=O)CCC(CCCOC(=O)c1ccccc1)c1cc(C)c2ccccc2n1</smiles>

13-Ethoxy-10-(4-methylquinolin-2-yl)-13-oxotridecyl benzoate. Colorless oil $(71.3 \mathrm{mg}, 71 \%$ yield $), 0.2 \mathrm{mmol}$ scale reaction. ${ }^{1} \mathbf{H}$ NMR $(500 \mathrm{MHz}$, chloroform- $d$ ) $\delta 8.12$ -7.99 (comp, 3H), $7.98-7.91(\mathrm{~m}, 1 \mathrm{H}), 7.68-7.64(\mathrm{~m}$, 1H), $7.57-7.46$ (comp, 2H), $7.44-7.40$ (comp, 2H), $7.10(\mathrm{~s}, 1 \mathrm{H}), 4.32-4.27$ (comp, 2H), $4.06-4.01$ (comp, $2 \mathrm{H}), 2.92-2.86(\mathrm{~m}, 1 \mathrm{H}), 2.68(\mathrm{~s}, 3 \mathrm{H}), 2.32-2.18(\mathrm{~m}, 1 \mathrm{H}), 2.17-2.06$ (comp, 3H), $1.78-1.68$ (comp, 4H), $1.43-1.35$ (comp, 2H), $1.31-1.16$ (comp, 13H). ${ }^{13}$ C NMR (126 MHz, chloroform-d) $\delta .173 .8,166.8,164.7,147.8,144.4,132.9,130.7,129.8$, 129.6, 129.0, 128.4, 127.2, 125.6, 123.7, 121.0, 65.2, 60.3, 48.2, 35.7, 32.6, 30.6, 29.8, 29.5, 29.5, 29.3, 28.8, 27.7, 26.1, 19.0, 14.3. HRMS (ESI) scaled for $[\mathrm{M}+\mathrm{H}]^{+}$: $\mathrm{C}_{32} \mathrm{H}_{42} \mathrm{NO}_{4}, \mathrm{~m} / \mathrm{z}: 504.3108$, observed: 504.3108 .<smiles>CCCCCCCCCC(CCC(=O)OCC)c1cc(C)c2ccccc2n1</smiles>

8-Ethoxy-5-(4-methylquinolin-2-yl)-8-oxooctyl furan-2-carboxylate. Colorless oil $(49.0 \mathrm{mg}, 58 \%$ yield), $0.2 \mathrm{mmol}$ scale reaction. ${ }^{1} \mathbf{H}$ NMR (500 $\mathrm{MHz}$, chloroform- $d$ ) $\delta 8.03(\mathrm{dd}, J=8.4,1.2 \mathrm{~Hz}$, $1 \mathrm{H}), 7.95$ (dd, $J=8.3,1.3 \mathrm{~Hz}, 1 \mathrm{H}), 7.67$ (ddd, $J=$ 8.4, 6.8, 1.3 Hz, 1H), $7.57-7.47$ (comp, 2H), 7.09 (s, 1H), $7.04(\mathrm{dd}, J=3.5,0.8 \mathrm{~Hz}, 1 \mathrm{H}), 6.44(\mathrm{dd}, J$ $=3.5,1.7 \mathrm{~Hz}, 1 \mathrm{H}), 4.24-4.19(\mathrm{~m}, 2 \mathrm{H}), 4.08-4.02(\mathrm{comp}, 2 \mathrm{H}), 2.95-2.89(\mathrm{~m}, 1 \mathrm{H})$, $2.67(\mathrm{~s}, 3 \mathrm{H}), 2.28-2.18(\mathrm{~m}, 1 \mathrm{H}), 2.17-2.06$ (comp, $2 \mathrm{H}), 1.92-1.86(\mathrm{~m}, 1 \mathrm{H}), 1.83-$ $1.63(\mathrm{comp}, 4 \mathrm{H}), 1.44-1.39(\mathrm{~m}, 1 \mathrm{H}), 1.32-1.23(\mathrm{~m}, 1 \mathrm{H}), 1.18(\mathrm{t}, J=7.1 \mathrm{~Hz}, 3 \mathrm{H}) .{ }^{13} \mathrm{C}$ NMR (126 MHz, chloroform-d) $\delta$ 173.7, 164.2, 158.9, 147.9, 146.3, 144.9, 144.6, $129.8,129.1,127.3,125.7,123.7,121.0,117.8,111.9,64.9,60.4,48.0,35.2,32.6,30.6$, 28.8, 24.0, 19.0, 14.3. HRMS (ESI) scaled for $[\mathrm{M}+\mathrm{H}]^{+}: \mathrm{C}_{25} \mathrm{H}_{30} \mathrm{NO}_{5}, \mathrm{~m} / \mathrm{z}: 424.2118$, observed: 424.2119 .

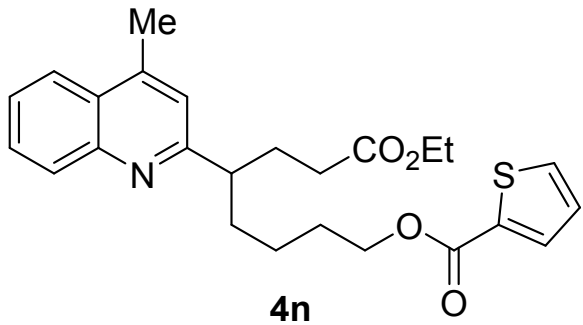

8-Ethoxy-5-(4-methylquinolin-2-yl)-8-oxooctyl thiophene-2-carboxylate. Colorless oil $(65.4 \mathrm{mg}$, $74 \%$ yield), $0.2 \mathrm{mmol}$ scale reaction. ${ }^{1} \mathbf{H}$ NMR (500 MHz, chloroform- $d$ ) $\delta 8.03(\mathrm{dd}, J=8.5,1.2$ $\mathrm{Hz}, 1 \mathrm{H}), 7.94(\mathrm{dd}, J=8.3,1.4 \mathrm{~Hz}, 1 \mathrm{H}), 7.71-$ 7.62 (comp, 2H), 7.55 - 7.44 (comp, 2H), 7.09 (s, S11 
1H), 7.03 (dd, $J=5.0,3.7 \mathrm{~Hz}, 1 \mathrm{H}), 4.24-4.17$ (comp, 2H), $4.07-4.01$ (comp, 2H), $2.96-2.90(\mathrm{~m}, 1 \mathrm{H}), 2.66(\mathrm{~s}, 3 \mathrm{H}), 2.30-2.20(\mathrm{~m}, 1 \mathrm{H}), 2.19-2.08(\mathrm{comp}, 2 \mathrm{H}), 1.94-$ 1.87 (m, 1H), $1.85-1.62$ (comp, 4H), $1.49-1.38$ (m, 1H), $1.32-1.23$ (m, 1H), 1.18 $(\mathrm{t}, J=7.1 \mathrm{~Hz}, 3 \mathrm{H}) .{ }^{13} \mathrm{C}$ NMR $(126 \mathrm{MHz}$, chloroform- $d$ ) $\delta 173.7,164.2,162.3,147.9$, $144.5,134.1,133.3,132.2,129.8,129.1,127.7,127.3,125.7,123.7,121.0,65.0,60.3$, 48.0, 35.2, 32.6, 30.6, 28.8, 24.0, 19.0, 14.3. HRMS (ESI) scaled for $[\mathrm{M}+\mathrm{H}]^{+}$: $\mathrm{C}_{25} \mathrm{H}_{30} \mathrm{NO}_{4} \mathrm{~S}, \mathrm{~m} / \mathrm{z}: 440.1890$, observed: 440.1890 .

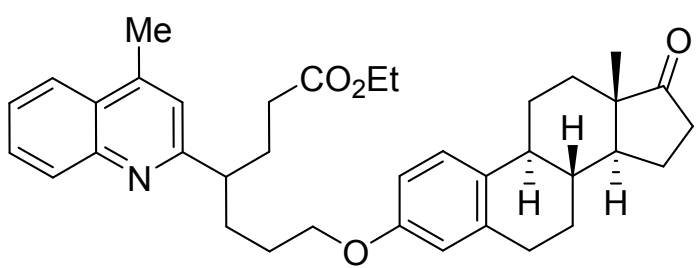

40:Estrone derivative

Ethyl 7-\{[(8R,9S,13S,14S)-13-methyl-17-oxo-7,8,9,11,12,13,14,15,16,17-decahydro-6H-cyclopenta $[\boldsymbol{a}]$ phenanthren-3-yl]oxy $\}$-4-(4-methylquinolin-2-

yl)heptanoate. Colorless oil ( $86.2 \mathrm{mg}, d r: 1: 1,76 \%$ yield), $0.2 \mathrm{mmol}$ scale reaction. The diastereoisomers couldn't be separately identified from the ${ }^{1} \mathrm{H}-\mathrm{NMR}$ spectrum; the $d r$ value was determined by HPLC (CHIRALPAK IC, hexane/2-propanol $=80 / 20$, flow rate $\left.1.0 \mathrm{~mL} / \mathrm{min}, \mathrm{T}=25{ }^{\circ} \mathrm{C}, 210 \mathrm{~nm}\right), \mathrm{t}_{\mathrm{R}}=52.79 \mathrm{~min}, \mathrm{t}_{\mathrm{R}}=61.99 \mathrm{~min} .{ }^{1} \mathbf{H} \mathbf{~ N M R}(500$ $\mathrm{MHz}$, chloroform- $d$ ) $\delta 8.04(\mathrm{dd}, J=8.4,1.3 \mathrm{~Hz}, 1 \mathrm{H}), 7.95(\mathrm{dd}, J=8.3,1.4 \mathrm{~Hz}, 1 \mathrm{H})$, $7.67(\mathrm{ddd}, J=8.4,6.8,1.3 \mathrm{~Hz}, 1 \mathrm{H}), 7.51(\mathrm{ddd}, J=8.3,6.8,1.4 \mathrm{~Hz}, 1 \mathrm{H}), 7.17-7.10$ (comp, 2H), 6.64 (dd, $J=8.7,2.9 \mathrm{~Hz}, 1 \mathrm{H}), 6.57-6.55$ (m, 1H), 4.08 - 4.02 (comp, $2 \mathrm{H}), 3.89-3.86$ (comp, 2H), $2.99-2.94(\mathrm{~m}, 1 \mathrm{H}), 2.92-2.78$ (comp, 2H), $2.68(\mathrm{~s}, 3 \mathrm{H})$, $2.54-2.45$ (m, 1H), $2.41-2.33(\mathrm{~m}, 1 \mathrm{H}), 2.28-2.20$ (comp, 2H), $2.18-2.09$ (comp, 4H), $2.06-2.03(\mathrm{~m}, 1 \mathrm{H}), 2.01-1.91$ (comp, 4H), $1.81-1.72(\mathrm{~m}, 1 \mathrm{H}), 1.66-1.59$ (comp, 2H), $1.55-1.36$ (comp, 5H), 1.19 (t, $J=7.1 \mathrm{~Hz}, 3 \mathrm{H}), 0.90(\mathrm{~s}, 3 \mathrm{H}) .{ }^{13} \mathbf{C}$ NMR (126 MHz, chloroform-d) $\delta 221.1,173.7,164.1,157.1,147.9,144.6,137.7,132.0$, $129.8,129.1,127.3,126.4,125.8,123.7,121.1,114.6,112.3,67.8,60.4,50.6,48.1$, 47.8, 44.1, 38.5, 36.0, 32.5, 32.0, 31.7, 30.7, 29.7, 27.5, 26.7, 26.0, 21.7, 19.0, 14.3, 14.0. HRMS (ESI) scaled for $[\mathrm{M}+\mathrm{H}]^{+}: \mathrm{C}_{37} \mathrm{H}_{46} \mathrm{NO}_{4}, \mathrm{~m} / \mathrm{z}: 568.3421$, observed: 568.3421 .<smiles>CCOC(=O)CCC(CNC(=O)c1ccccc1)c1cc(C)c2ccccc2n1</smiles>

Ethyl 5-benzamido-4-(4-methylquinolin-2-yl)pentanoate. Colorless oil ( $49.1 \mathrm{mg}, 63 \%$ yield), $0.2 \mathrm{mmol}$ scale reaction. ${ }^{1} \mathbf{H}$ NMR (500 MHz, chloroform- $d$ ) $\delta 8.04(\mathrm{dd}, J=8.5,1.2$ $\mathrm{Hz}, 1 \mathrm{H}$ ), $7.99-7.97$ (comp, 2H), $7.84-7.78$ (comp, 2H), 7.71 (ddd, $J=8.5,6.9,1.4 \mathrm{~Hz}, 1 \mathrm{H}), 7.55$ (ddd, $J=8.2,6.9$, $1.2 \mathrm{~Hz}, 1 \mathrm{H}), 7.50-7.43(\mathrm{~m}, 1 \mathrm{H}), 7.45-7.37$ (comp, 2H), $7.19(\mathrm{~s}, 1 \mathrm{H}), 4.10-4.05(\mathrm{comp}, 3 \mathrm{H}), 3.77-3.73(\mathrm{~m}, 1 \mathrm{H})$, $3.31-3.26(\mathrm{~m}, 1 \mathrm{H}), 2.69(\mathrm{~s}, 3 \mathrm{H}), 2.37-2.34$ (comp, $2 \mathrm{H})$, $2.18-2.12(\mathrm{~m}, 1 \mathrm{H}), 2.29-2.20(\mathrm{~m}, 1 \mathrm{H}), 1.21(\mathrm{t}, J=7.1 \mathrm{~Hz}, 3 \mathrm{H}) .{ }^{13} \mathbf{C}$ NMR $(126 \mathrm{MHz}$, chloroform- $d$ ) $\delta 173.4,167.5,162.9,147.5,145.5,134.9,131.3,129.6,129.5,128.6$, 127.3, 127.0, 126.2, 123.9, 122.4, 60.5, 45.4, 42.6, 32.1, 27.9, 18.9, 14.3. HRMS (ESI) scaled for $[\mathrm{M}+\mathrm{H}]^{+}: \mathrm{C}_{24} \mathrm{H}_{27} \mathrm{~N}_{2} \mathrm{O}_{3}, \mathrm{~m} / \mathrm{z}$ : 391.2016, observed: 391.2018.<smiles>CCOC(=O)CCC(CCCN1C(=O)c2ccccc2C1=O)c1cc(C)c2ccccc2n1</smiles> 
yield), 0.2 mmol scale reaction. ${ }^{1} \mathbf{H}$ NMR $(500 \mathrm{MHz}$, chloroform- $d$ ) $\delta 7.97$ (dd, $J=8.4$, $1.2 \mathrm{~Hz}, 1 \mathrm{H}), 7.93(\mathrm{dd}, J=8.3,1.4 \mathrm{~Hz}, 1 \mathrm{H}), 7.79(\mathrm{dd}, J=5.5,3.1 \mathrm{~Hz}, 2 \mathrm{H}), 7.67(\mathrm{dd}, J$ $=5.5,3.1 \mathrm{~Hz}, 2 \mathrm{H}), 7.64(\mathrm{ddd}, J=8.4,6.8,1.4 \mathrm{~Hz}, 1 \mathrm{H}), 7.49$ (ddd, $J=8.3,6.8,1.2 \mathrm{~Hz}$, $1 \mathrm{H}), 7.07(\mathrm{~d}, J=1.1 \mathrm{~Hz}, 1 \mathrm{H}), 4.06-4.00(\mathrm{comp}, 2 \mathrm{H}), 3.64(\mathrm{t}, J=7.1 \mathrm{~Hz}, 2 \mathrm{H}), 2.93(\mathrm{tt}$, $J=9.1,5.3 \mathrm{~Hz}, 1 \mathrm{H}), 2.66(\mathrm{~s}, 3 \mathrm{H}), 2.26-2.17(\mathrm{~m}, 1 \mathrm{H}), 2.15-2.06$ (comp, 3H), $1.94-$ $1.84(\mathrm{~m}, 1 \mathrm{H}), 1.81-1.74(\mathrm{~m}, 1 \mathrm{H}), 1.73-1.64(\mathrm{~m}, 1 \mathrm{H}), 1.51(\mathrm{dtd}, J=13.2,7.4,3.5 \mathrm{~Hz}$, $1 \mathrm{H}), 1.17$ (t, $J=7.1 \mathrm{~Hz}, 3 \mathrm{H}) .{ }^{13} \mathbf{C}$ NMR $(126 \mathrm{MHz}$, chloroform- $d$ ) $\delta 173.6,168.4,163.7$, 147.9, 144.6, 133.9, 132.2, 129.8, 129.1, 127.3, 125.7, 123.7, 123.3, 121.2, 60.3, 47.7, $38.1,32.7,32.5,30.5,26.8,18.9,14.3$. HRMS (ESI) scaled for $[\mathrm{M}+\mathrm{H}]^{+}: \mathrm{C}_{27} \mathrm{H}_{29} \mathrm{~N}_{2} \mathrm{O}_{4}$, $\mathrm{m} / \mathrm{z}: 445.2122$, observed: 445.2122 .

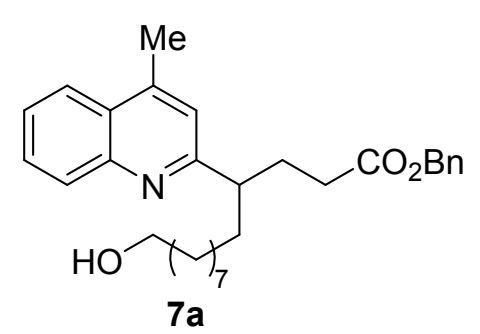

Benzyl 13-hydroxy-4-(4-methylquinolin-2-yl)tridecanoate. Colorless oil ( $64.6 \mathrm{mg}, 70 \%$ yield), $0.2 \mathrm{mmol}$ scale reaction. ${ }^{1} \mathbf{H}$ NMR $(500 \mathrm{MHz}$, chloroform- $d$ ) $\delta 8.04$ (dd, $J=8.4,1.2 \mathrm{~Hz}, 1 \mathrm{H}), 7.94(\mathrm{dd}, J=8.3,1.4 \mathrm{~Hz}, 1 \mathrm{H})$, 7.66 (ddd, $J=8.4,6.8,1.4 \mathrm{~Hz}, 1 \mathrm{H}), 7.50$ (ddd, $J=8.3$, 6.8, $1.2 \mathrm{~Hz}, 1 \mathrm{H}), 7.34-7.27$ (comp, 5H), 7.07 (s, 1H), $5.06(\mathrm{~d}, J=12.4 \mathrm{~Hz}, 1 \mathrm{H}), 5.02(\mathrm{~d}, J=12.4 \mathrm{~Hz}, 1 \mathrm{H}), 3.60$ $(\mathrm{t}, J=6.6 \mathrm{~Hz}, 2 \mathrm{H}), 2.89(\mathrm{tt}, J=8.6,5.9 \mathrm{~Hz}, 1 \mathrm{H}), 2.67(\mathrm{~s}, 3 \mathrm{H}), 2.34-2.27(\mathrm{~m}, 1 \mathrm{H}), 2.23$ $-2.18(\mathrm{~m}, 1 \mathrm{H}), 2.17-2.08$ (comp, $2 \mathrm{H}), 1.83-1.76(\mathrm{~m}, 1 \mathrm{H}), 1.75-1.64$ (comp, 2H), $1.59-1.47$ (comp, 3H), $1.32-1.17$ (comp, 10H). ${ }^{13} \mathbf{C}$ NMR (126 MHz, chloroform-d) $\delta$ 173.6, 164.6, 147.8, 144.5, 136.1, 129.7, 129.1, 128.6, 128.3, 128.2, 127.2, 125.7, 123.7, 121.0, 66.2, 63.1, 48.1, 35.7, 32.9, 32.6, 30.5, 29.7, 29.6, 29.4, 27.6, 25.8, 19.0. Peak overlapping was observed. HRMS (ESI) scaled for $[\mathrm{M}+\mathrm{H}]^{+}: \mathrm{C}_{30} \mathrm{H}_{40} \mathrm{NO}_{3}, \mathrm{~m} / \mathrm{z}$ : 462.3003, observed: 462.3003 .

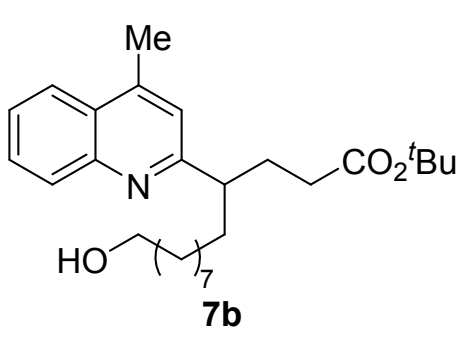

tert-Butyl 13-hydroxy-4-(4-methylquinolin-2-yl)tridecanoate. Colorless oil ( $71.7 \mathrm{mg}, 84 \%$ yield $), 0.2 \mathrm{mmol}$ scale reaction. ${ }^{1} \mathbf{H}$ NMR $(500 \mathrm{MHz}$, chloroform- $d$ ) $\delta 8.05$ $(\mathrm{dd}, J=8.4,1.3 \mathrm{~Hz}, 1 \mathrm{H}), 7.94(\mathrm{dd}, J=8.2,1.5 \mathrm{~Hz}, 1 \mathrm{H})$, 7.66 (ddd, $J=8.4,6.8,1.5 \mathrm{~Hz}, 1 \mathrm{H}), 7.50(\mathrm{ddd}, J=8.2$, $6.8,1.3 \mathrm{~Hz}, 1 \mathrm{H}), 7.09(\mathrm{~s}, 1 \mathrm{H}), 3.60(\mathrm{t}, J=6.7 \mathrm{~Hz}, 2 \mathrm{H})$, $2.94-2.82(\mathrm{~m}, 1 \mathrm{H}), 2.68(\mathrm{~s}, 3 \mathrm{H}), 2.18-2.11(\mathrm{~m}, 1 \mathrm{H})$, $2.07-1.99$ (comp, 3H), $1.83-1.66$ (comp, 3H), $1.57-1.46$ (comp, 4H), 1.38 (s, 9H), 1.31 - 1.18 (comp, 9H). ${ }^{13}$ C NMR (126 MHz, chloroform- $d$ ) $\delta$ 173.2, 164.9, 147.8, 144.4, 129.7, 129.0, 127.2, 125.6, 123.7, 121.0, 80.1, 63.1, 48.2, 35.7, 33.8, 32.9, 30.7, 29.8, 29.6, 29.4, 28.2, 27.7, 25.8, 19.0. Peak overlapping was observed. HRMS (ESI) scaled for $[\mathrm{M}+\mathrm{H}]^{+}: \mathrm{C}_{27} \mathrm{H}_{42} \mathrm{NO}_{3}, \mathrm{~m} / \mathrm{z}$ : 428.3159, observed: 428.3157 .

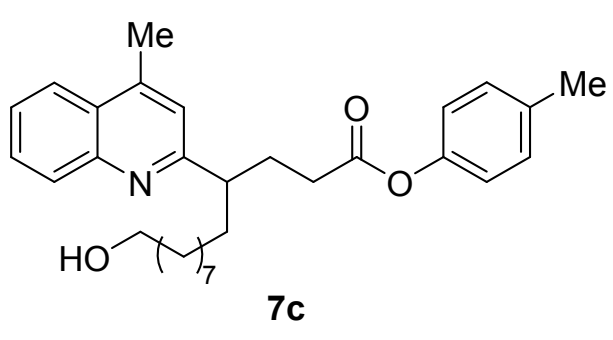

p-Tolyl 13-hydroxy-4-(4-methylquinolin-2yl)tridecanoate. Colorless oil $(70.0 \mathrm{mg}, 76 \%$ yield), $0.2 \mathrm{mmol}$ scale reaction. ${ }^{1} \mathbf{H}$ NMR (500 $\mathrm{MHz}$, chloroform- $d$ ) $\delta 8.08(\mathrm{dd}, J=8.4,1.2 \mathrm{~Hz}$, $1 \mathrm{H}), 7.97$ (dd, $J=8.3,1.3 \mathrm{~Hz}, 1 \mathrm{H}), 7.68$ (ddd, $J$ $=8.4,6.8,1.3 \mathrm{~Hz}, 1 \mathrm{H}), 7.52(\mathrm{ddd}, J=8.3,6.8$, $1.2 \mathrm{~Hz}, 1 \mathrm{H}), 7.14-7.09$ (comp, 3H), $6.90-6.84$ (m, 2H), $3.60(\mathrm{t}, J=6.7 \mathrm{~Hz}, 2 \mathrm{H}), 2.99(\mathrm{tt}, J=8.7,6.0 \mathrm{~Hz}, 1 \mathrm{H}), 2.70(\mathrm{~s}, 3 \mathrm{H}), 2.53-2.46$ 
(m, 1H), $2.42-2.35(\mathrm{~m}, 1 \mathrm{H}), 2.31(\mathrm{~s}, 3 \mathrm{H}), 2.24-2.19$ (comp, 2H), $1.89-1.81(\mathrm{~m}, 1 \mathrm{H})$, $1.80-1.73$ (m, 1H), $1.56-1.49$ (comp, 2H), $1.34-1.10$ (comp, 12H). ${ }^{13}$ C NMR (126 $\mathrm{MHz}$, chloroform- $d$ ) $\delta 172.5,164.5,148.6,147.8,144.7,135.4,129.9,129.7,129.2$, $127.3,125.7,123.7,121.3,121.0,63.1,48.1,35.7,32.9,32.7,30.5,29.7,29.6,29.44$, 29.43, 27.7, 25.8, 20.9, 19.0. HRMS (ESI) scaled for $[\mathrm{M}+\mathrm{H}]^{+}: \mathrm{C}_{30} \mathrm{H}_{40} \mathrm{NO}_{3}, \mathrm{~m} / \mathrm{z}$ : 462.3003, observed: 462.2999 .

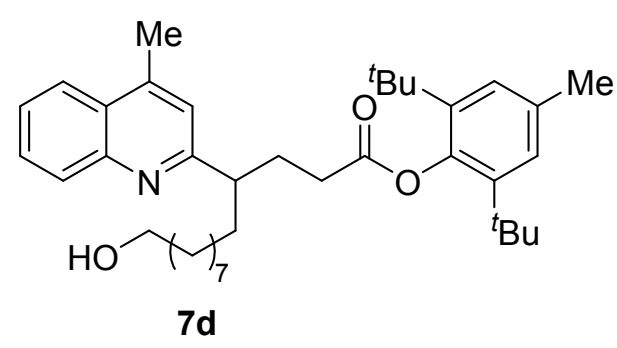

2,6-Di-tert-butyl-4-methylphenyl 13-hydroxy-4-(4-methylquinolin-2-yl)tridecanoate. Colorless oil (101.1 mg, 88\% yield), $0.2 \mathrm{mmol}$ scale reaction. ${ }^{1} \mathbf{H}$ NMR $(500 \mathrm{MHz}$, chloroformd) $\delta 8.08(\mathrm{dd}, J=8.4,1.2 \mathrm{~Hz}, 1 \mathrm{H}), 7.98(\mathrm{dd}, J=$ $8.3,1.4 \mathrm{~Hz}, 1 \mathrm{H}), 7.70$ (ddd, $J=8.4,6.8,1.4 \mathrm{~Hz}$, $1 \mathrm{H}), 7.53$ (ddd, $J=8.3,6.8,1.2 \mathrm{~Hz}, 1 \mathrm{H}), 7.14$ (s, $1 \mathrm{H}), 7.08(\mathrm{~s}, 2 \mathrm{H}), 3.61(\mathrm{t}, J=6.7 \mathrm{~Hz}, 2 \mathrm{H}), 3.06-2.94(\mathrm{~m}, 1 \mathrm{H}), 2.71(\mathrm{~s}, 3 \mathrm{H}), 2.60(\mathrm{ddd}$, $J=17.0,10.0,6.6 \mathrm{~Hz}, 1 \mathrm{H}), 2.45(\mathrm{ddd}, J=17.0,10.0,5.5 \mathrm{~Hz}, 1 \mathrm{H}), 2.30$ (s, 3H), $2.30-$ 2.19 (m, 2H), $1.88-1.74$ (comp, 2H), $1.57-1.48$ (comp, 3H), $1.36-1.29$ (comp, 6H), 1.28 (s, 9H), 1.24 (s, 9H), $1.24-1.19$ (comp, 5H). ${ }^{13}$ C NMR (126 MHz, chloroformd) $\delta 173.9,164.6,147.9,145.9,144.5,142.0,134.5,129.8,129.1,127.12,127.08,125.7$, $123.7,121.1,63.1,48.0,36.0,35.3,33.9,32.9,31.60,31.56,29.8,29.7,29.6,29.5,27.6$, 25.8, 21.6, 19.0. HRMS (ESI) scaled for $[\mathrm{M}+\mathrm{H}]^{+}: \mathrm{C}_{38} \mathrm{H}_{56} \mathrm{NO}_{3}, \mathrm{~m} / \mathrm{z}: 574.4255$, observed: 574.4247 .

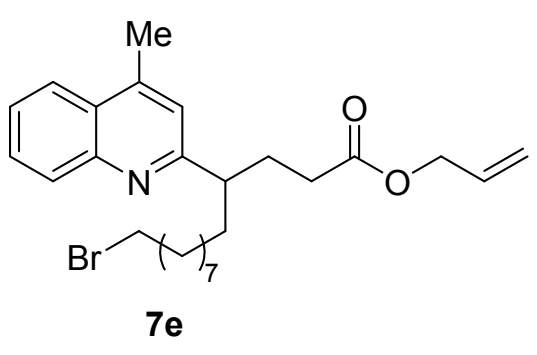

Allyl 13-bromo-4-(4-methylquinolin-2-yl)tridecanoate. Colorless oil ( $58.7 \mathrm{mg}, 62 \%$ yield), $0.2 \mathrm{mmol}$ scale reaction. ${ }^{1} \mathbf{H}$ NMR $(500 \mathrm{MHz}$, chloroform- $d$ ) $\delta$ $8.04(\mathrm{dd}, J=8.4,1.3 \mathrm{~Hz}, 1 \mathrm{H}), 7.95(\mathrm{dd}, J=8.4,1.4$ $\mathrm{Hz}, 1 \mathrm{H}), 7.67$ (ddd, $J=8.4,6.8,1.4 \mathrm{~Hz}, 1 \mathrm{H}), 7.51$ (ddd, $J=8.4,6.8,1.3 \mathrm{~Hz}, 1 \mathrm{H}), 7.09(\mathrm{~s}, 1 \mathrm{H}), 5.85$ (ddt, $J=17.3,10.5,5.7 \mathrm{~Hz}, 1 \mathrm{H}), 5.27-5.23(\mathrm{~m}, 1 \mathrm{H}), 5.19$ $-5.17(\mathrm{~m}, 1 \mathrm{H}), 4.54-4.45$ (comp, 2H), 3.37 (t, $J=6.9 \mathrm{~Hz}, 2 \mathrm{H}), 2.89$ (tt, $J=8.6,6.0$ $\mathrm{Hz}, 1 \mathrm{H}), 2.68$ (s, 3H), $2.32-2.26(\mathrm{~m}, 1 \mathrm{H}), 2.22-2.14(\mathrm{~m}, 1 \mathrm{H}), 2.13-2.07$ (comp, 2H), $1.89-1.76$ (comp, 3H), $1.74-1.66$ (m, 1H), $1.38-1.33$ (comp, 2H), $1.40-1.11$ (comp, 10H). ${ }^{13} \mathbf{C}$ NMR (126 MHz, chloroform- $d$ ) $\delta$ 173.4, 164.6, 147.8, 144.4, 132.4, 129.8, 129.1, 127.2, 125.7, 123.7, 121.0, 118.2, 65.1, 48.2, 35.7, 34.2, 32.9, 32.5, 30.5, 29.7, 29.42, 29.39, 28.8, 28.3, 27.6, 19.0. Peak overlapping was observed. HRMS (ESI) scaled for $[\mathrm{M}+\mathrm{H}]^{+}: \mathrm{C}_{26} \mathrm{H}_{37} \mathrm{BrNO}_{2}, \mathrm{~m} / \mathrm{z}$ : 474.2002, observed: 474.1994 .

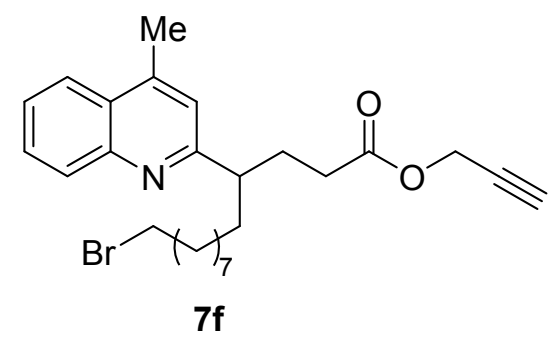

Prop-2-yn-1-yl 13-bromo-4-(4-methylquinolin-2yl)tridecanoate. Colorless oil (63.4 mg, 67\% yield), 0.2 mmol scale reaction. ${ }^{1} \mathbf{H}$ NMR $(500 \mathrm{MHz}$, chloroform- $d$ ) $\delta 8.04(\mathrm{dd}, J=8.5,1.2 \mathrm{~Hz}, 1 \mathrm{H}), 7.95$ $(\mathrm{dd}, J=8.2,1.4 \mathrm{~Hz}, 1 \mathrm{H}), 7.67$ (ddd, $J=8.5,6.8,1.4$ $\mathrm{Hz}, 1 \mathrm{H}), 7.51$ (ddd, $J=8.2,6.8,1.2 \mathrm{~Hz}, 1 \mathrm{H}), 7.09$ (s, $1 \mathrm{H}), 4.59(\mathrm{~d}, J=2.4 \mathrm{~Hz}, 2 \mathrm{H}), 3.37(\mathrm{t}, J=6.9 \mathrm{~Hz}$, 2H), 2.89 (ddt, $J=8.7,7.5,6.0 \mathrm{~Hz}, 1 \mathrm{H}), 2.69(\mathrm{~s}, 3 \mathrm{H}), 2.42$ (t, $J=2.4 \mathrm{~Hz}, 1 \mathrm{H}), 2.35-$ $2.27(\mathrm{~m}, 1 \mathrm{H}), 2.21(\mathrm{dd}, J=8.0,7.1 \mathrm{~Hz}, 1 \mathrm{H}), 2.14-2.07$ (comp, 2H), $1.85-1.76$ (comp, 
4H), $1.73-1.68$ (m, 1H), $1.39-1.32$ (comp, 2H), $1.29-1.20$ (comp, 9H). ${ }^{13}$ C NMR (126 MHz, chloroform-d) $\delta 172.9,164.4,147.8,144.5,129.8,129.1,127.2,125.7$, 123.7, 121.0, 77.9, 74.8, 51.9, 48.1, 35.7, 34.2, 32.9, 32.3, 30.4, 29.7, 29.42, 29.40, 28.8, 28.3, 27.6, 19.0. HRMS (ESI) scaled for $[\mathrm{M}+\mathrm{H}]^{+}: \mathrm{C}_{26} \mathrm{H}_{35} \mathrm{BrNO}_{2}, \mathrm{~m} / \mathrm{z}: 472.1846$, observed: 472.1827.

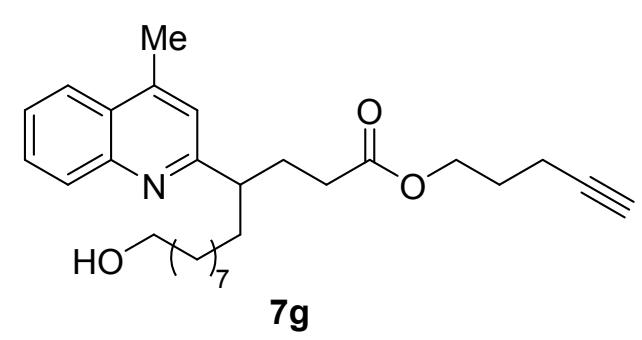

Pent-4-yn-1-yl 13-hydroxy-4-(4-methylquinolin-2-yl)tridecanoate. Colorless oil (50.1 mg, $57 \%$ yield), $0.2 \mathrm{mmol}$ scale reaction. ${ }^{1} \mathbf{H}$ NMR (500 MHz, chloroform- $d$ ) $\delta 8.04(\mathrm{dd}, J=8.5$, $1.2 \mathrm{~Hz}, 1 \mathrm{H}), 7.95(\mathrm{dd}, J=8.3,1.4 \mathrm{~Hz}, 1 \mathrm{H}), 7.66$ (ddd, $J=8.5,6.8,1.4 \mathrm{~Hz}, 1 \mathrm{H}), 7.50$ (ddd, $J=$ 8.3, 6.8, $1.2 \mathrm{~Hz}, 1 \mathrm{H}), 7.09(\mathrm{~s}, 1 \mathrm{H}), 4.13-4.02$ (comp, 2H), 3.60 (t, $J=6.7 \mathrm{~Hz}, 2 \mathrm{H}), 2.88(\mathrm{tt}, J=8.8,5.8 \mathrm{~Hz}, 1 \mathrm{H}), 2.68(\mathrm{~s}, 3 \mathrm{H}), 2.21$ (td, $J=7.0,2.6 \mathrm{~Hz}, 2 \mathrm{H}), 2.12-2.06$ (comp, 2H), 1.93 (t, $J=2.6 \mathrm{~Hz}, 1 \mathrm{H}), 1.80-1.75$ (comp, 3H), 1.56 - 1.47 (comp, 2H), 1.33 - 1.18 (comp, 15H). ${ }^{13}$ C NMR (126 MHz, chloroform- $d$ ) $\delta 173.7,164.6,147.8,144.5,129.7,129.1,127.2,125.7,123.7,121.0$, 83.2, 69.1, 63.1, 62.9, 48.2, 35.7, 32.9, 32.5, 30.5, 29.7, 29.5, 29.4, 27.6, 25.8, 19.0, 15.3. Peak overlapping was observed. HRMS (ESI) scaled for $[\mathrm{M}+\mathrm{H}]^{+}: \mathrm{C}_{28} \mathrm{H}_{40} \mathrm{NO}_{3}$, m/z: 438.3003, observed: 438.2999.<smiles>COc1cc(CCCOC(=O)CCC(CCC(Br)(Br)C(=O)O)c2cc(C)c3ccccc3n2)ccc1OCc1ccccc1</smiles>

3-[4-(Benzyloxy)-3-methoxyphenyl]propyl 13-bromo-4-(4-methylquinolin-2-yl)tridecanoate. Colorless oil (103.4 mg, 75\% yield), $0.2 \mathrm{mmol}$ scale reaction. ${ }^{1} \mathbf{H}$ NMR $(500 \mathrm{MHz}$, chloroform- $d$ ) $\delta 8.05(\mathrm{dd}, J=8.4,1.3$ $\mathrm{Hz}, 1 \mathrm{H}), 7.95(\mathrm{dd}, J=8.4,1.4 \mathrm{~Hz}, 1 \mathrm{H})$, 7.67 (ddd, $J=8.3,6.8,1.4 \mathrm{~Hz}, 1 \mathrm{H}$ ), 7.51 (ddd, $J=8.3,6.8,1.3 \mathrm{~Hz}, 1 \mathrm{H}), 7.46-7.40$ (comp, 2H), $7.39-7.32$ (comp, 2H), $7.32-7.25(\mathrm{~m}, 1 \mathrm{H}), 7.10(\mathrm{~s}, 1 \mathrm{H}), 6.78(\mathrm{~d}, J=8.1 \mathrm{~Hz}, 1 \mathrm{H}), 6.69(\mathrm{~d}, J=2.1 \mathrm{~Hz}, 1 \mathrm{H})$, $6.61(\mathrm{dd}, J=8.1,2.1 \mathrm{~Hz}, 1 \mathrm{H}), 5.11(\mathrm{~s}, 2 \mathrm{H}), 4.07-3.94$ (comp, 2H), 3.85 (s, 3H), 3.37 (t, $J=6.9 \mathrm{~Hz}, 2 \mathrm{H}), 2.90(\mathrm{tt}, J=8.4,6.1 \mathrm{~Hz}, 1 \mathrm{H}), 2.68(\mathrm{~s}, 3 \mathrm{H}), 2.56(\mathrm{dd}, J=8.7,6.7 \mathrm{~Hz}$, $2 \mathrm{H}), 2.33-2.21(\mathrm{~m}, 1 \mathrm{H}), 2.21-2.06$ (comp, 3H), $1.90-1.68$ (comp, 7H), $1.41-1.30$ (comp, 2H), 1.29 - 1.13 (comp, 9H). ${ }^{13} \mathbf{C}$ NMR (126 MHz, chloroform- $d$ ) $\delta 173.8$, 164.6, 149.7, 147.8, 146.6, 144.4, 137.5, 134.6, 129.8, 129.1, 128.6, 127.9, 127.4, 127.2, $125.7,123.7,121.0,120.3,114.4,112.4,71.3,63.7,56.1,48.3,35.8,34.2,32.9,32.6$, $31.8,30.6,30.4,29.8,29.4,28.8,28.2,27.7,19.0$. Peak overlapping was observed. HRMS (ESI) scaled for $[\mathrm{M}+\mathrm{H}]^{+}: \mathrm{C}_{40} \mathrm{H}_{51} \mathrm{BrNO}_{4}, \mathrm{~m} / \mathrm{z}$ : 688.2996, observed: 688.2978.

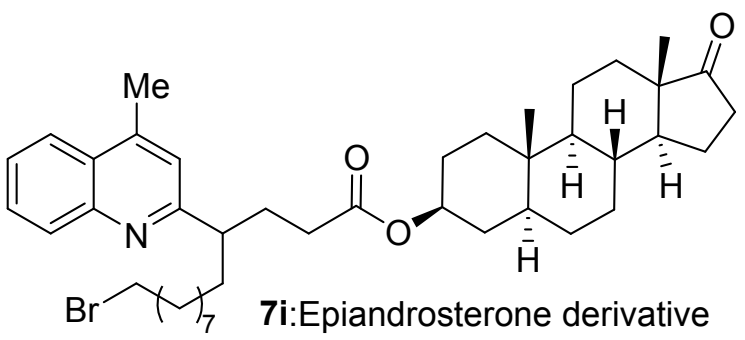


$(3 S, 5 S, 8 R, 9 S, 10 S, 13 S, 14 S)-10,13-D i-m e t h y l-17-0 x o h e x a d e c a h y d r o-1 H$ -

cyclopenta $[a]$ phenanthren-3-yl 13-bromo-4-(4-methylquinolin-2-yl)tridecanoate. Colorless oil (101.9 mg, $d r: 1: 1,72 \%$ yield), $0.2 \mathrm{mmol}$ scale reaction. The diastereoisomers couldn't be separately identified from the ${ }^{1} \mathrm{H}-\mathrm{NMR}$ spectrum; the $d r$ value was determined by HPLC (CHIRALPAK AD, hexane/2-propanol = 90/10, flow rate $\left.1.0 \mathrm{~mL} / \mathrm{min}, \mathrm{T}=25^{\circ} \mathrm{C}, 210 \mathrm{~nm}\right), \mathrm{t}_{\mathrm{R}}=8.14 \mathrm{~min}, \mathrm{t}_{\mathrm{R}}=9.59 \mathrm{~min} .{ }^{1} \mathbf{H} \mathbf{N M R}(500 \mathrm{MHz}$, chloroform- $d$ ) $\delta 8.03(\mathrm{dd}, J=8.4,1.2 \mathrm{~Hz}, 1 \mathrm{H}), 7.94(\mathrm{dd}, J=8.3,1.3 \mathrm{~Hz}, 1 \mathrm{H}), 7.66$ (ddd, $J=8.4,6.8,1.3 \mathrm{~Hz}, 1 \mathrm{H}), 7.50(\mathrm{ddd}, J=8.3,6.8,1.2 \mathrm{~Hz}, 1 \mathrm{H}), 7.08(\mathrm{~s}, 1 \mathrm{H}), 4.65-4.58$ $(\mathrm{m}, 1 \mathrm{H}), 3.36(\mathrm{t}, J=6.9 \mathrm{~Hz}, 2 \mathrm{H}), 2.91-2.83(\mathrm{~m}, 1 \mathrm{H}), 2.68(\mathrm{~s}, 3 \mathrm{H}), 2.45-2.37(\mathrm{~m}$, $1 \mathrm{H}), 2.26-2.15(\mathrm{~m}, 1 \mathrm{H}), 2.13-2.00$ (comp, $4 \mathrm{H}), 1.94-1.88$ (comp, $2 \mathrm{H}), 1.83-1.73$ (comp, 6H), 1.72 - 1.66 (comp, 2H), $1.62-1.42$ (comp, 6H), $1.39-1.17$ (comp, 17H), $1.02-0.91$ (comp, 2H), 0.83 (s, 3H), $0.80(\mathrm{~s}, 3 \mathrm{H}), 0.71-0.69(\mathrm{~m}, 1 \mathrm{H}) .{ }^{13} \mathbf{C}$ NMR $(126$ $\mathrm{MHz}$, chloroform- $d$ ) $\delta 221.3,173.3,164.7,147.8,144.3,129.8,129.0,127.2,125.6$, 123.7, 121.1, 73.4, 54.4, 51.5, 48.2, 47.9, 44.7, 36.8, 36.0, 35.74, 35.66, 35.1, 34.1, 34.0, 33.0, 32.9, 31.6, 30.9, 30.6, 29.7, 29.4, 28.8, 28.4, 28.2, 27.6, 27.52, 27.48, 21.9, 20.6, 19.0, 13.9, 12.3. HRMS (ESI) scaled for $[\mathrm{M}+\mathrm{H}]^{+}: \mathrm{C}_{42} \mathrm{H}_{61} \mathrm{BrNO}_{3}, \mathrm{~m} / \mathrm{z}: 706.3829$, observed: 706.3812 .

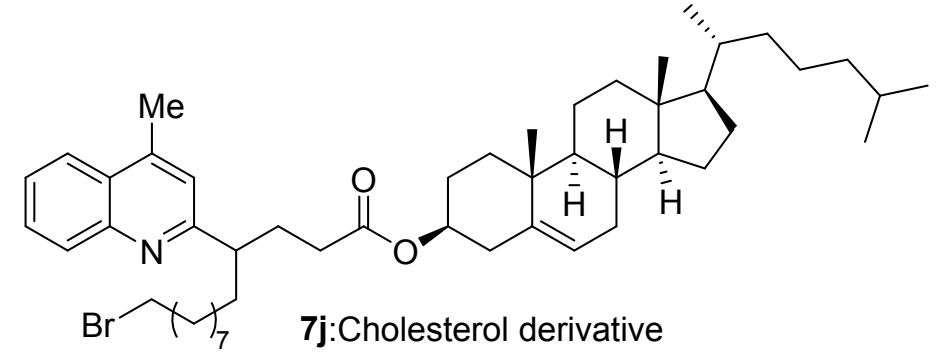

$(3 S, 8 S, 9 S, 10 R, 13 R, 14 S, 17 R)-10,13-D i m e t h y l-17-[(R)-6-m e t h y l h e p t a n-2-y l]-$ $2,3,4,7,8,9,10,11,12,13,14,15,16,17$-tetradecahydro- $1 H$-cyclopenta[a]-

phenanthren-3-yl 13-bromo-4-(4-methylquinolin-2-yl)tridecanoate. Colorless oil (128.6 mg, $d r: 1: 1,80 \%$ yield), $0.2 \mathrm{mmol}$ scale reaction. The $d r$ value was determined by analogy with $7 \mathbf{i} .{ }^{1} \mathbf{H}$ NMR $(500 \mathrm{MHz}$, chloroform- $d$ ) $\delta 8.07(\mathrm{dd}, J=8.4,1.2 \mathrm{~Hz}, 1 \mathrm{H})$, $7.97(\mathrm{dd}, J=8.3,1.4 \mathrm{~Hz}, 1 \mathrm{H}), 7.69(\mathrm{ddd}, J=8.4,6.8,1.4 \mathrm{~Hz}, 1 \mathrm{H}), 7.52$ (ddd, $J=8.3$, $6.8,1.2 \mathrm{~Hz}, 1 \mathrm{H}), 7.12(\mathrm{~s}, 1 \mathrm{H}), 5.43-5.27(\mathrm{~m}, 1 \mathrm{H}), 4.62-4.50(\mathrm{~m}, 1 \mathrm{H}), 3.39(\mathrm{t}, J=6.9$ $\mathrm{Hz}, 2 \mathrm{H}), 2.96-2.86(\mathrm{~m}, 1 \mathrm{H}), 2.70(\mathrm{~s}, 3 \mathrm{H}), 2.26-2.22$ (comp, 2H), $2.18-2.07$ (comp, 3H), $2.04-1.94$ (comp, 3H), $1.88-1.79$ (comp, 6H), $1.77-1.69$ (m, 1H), $1.64-1.42$ (comp, 8H), $1.42-1.33$ (comp, 5H), 1.33 - 1.21 (comp, 6H), $1.20-1.05$ (comp, 9H), 1.00 (s, 3H), $0.95-0.92$ (comp, 5H), $0.90-0.86$ (comp, 9H), 0.69 (s, 3H). ${ }^{13}$ C NMR (126 MHz, chloroform-d) $\delta 173.2,164.7,147.8,144.3,139.8,129.8,129.0,127.2$, 125.6, 123.7, 122.7, 121.1, 73.9, 56.8, 56.3, 50.1, 48.2, 42.4, 39.8, 39.6, 38.24, 38.19, $37.1,36.7,36.3,35.9,35.7,34.1,32.9,32.01,31.97,30.6,29.7,29.4,28.8,28.3,28.2$, 28.1, 27.9, 27.8, 27.7, 24.4, 24.0, 22.9, 22.7, 21.1, 19.4, 19.0, 18.8, 12.0. HRMS (ESI) scaled for $[\mathrm{M}+\mathrm{H}]^{+}: \mathrm{C}_{50} \mathrm{H}_{77} \mathrm{BrNO}_{2}, \mathrm{~m} / \mathrm{z}$ : 802.5132, observed: 802.5113.<smiles>CCN(CC)C(=O)CCC(CC(C)CO)c1cc(C)c2ccccc2n1</smiles>

$\mathrm{N}, \mathrm{N}$-Diethyl-13-hydroxy-4-(4-methylquinolin-2-yl)tridecanamide. Colorless oil (48.9 mg, $57 \%$ yield), 0.2 mmol scale reaction. ${ }^{1} \mathbf{H}$ NMR $(500 \mathrm{MHz}$, chloroformd) $\delta 8.03(\mathrm{dd}, J=8.4,1.2 \mathrm{~Hz}, 1 \mathrm{H}), 7.96(\mathrm{dd}, J=8.2$, 
$1.4 \mathrm{~Hz}, 1 \mathrm{H}), 7.67(\mathrm{ddd}, J=8.4,6.8,1.4 \mathrm{~Hz}, 1 \mathrm{H}), 7.51(\mathrm{ddd}, J=8.2,6.8,1.2 \mathrm{~Hz}, 1 \mathrm{H})$, $7.14(\mathrm{~s}, 1 \mathrm{H}), 3.62-3.59$ (comp, 2H), $3.39-3.28(\mathrm{~m}, 1 \mathrm{H}), 3.28-3.22(\mathrm{~m}, 1 \mathrm{H}), \quad 3.19$ - $3.12(\mathrm{~m}, 1 \mathrm{H}), 3.10-3.05(\mathrm{~m}, 1 \mathrm{H}), 2.91(\mathrm{tt}, J=8.5,5.7 \mathrm{~Hz}, 1 \mathrm{H}), 2.68(\mathrm{~s}, 3 \mathrm{H}), 2.25-$ $2.20(\mathrm{~m}, 1 \mathrm{H}), 2.17-2.02$ (comp, 3H), $1.81-1.68$ (comp, 2H), $1.57-1.48$ (comp, 2H), $1.34-1.15$ (comp, 12H), 1.04 (t, $J=7.1 \mathrm{~Hz}, 3 \mathrm{H}), 0.96$ (t, $J=7.1 \mathrm{~Hz}, 3 \mathrm{H}) .{ }^{13} \mathbf{C}$ NMR $(126 \mathrm{MHz}$, chloroform- $d$ ) $\delta 172.3,165.3,147.7,144.4,129.6,129.0,127.3,125.6$, 123.8, 121.0, 63.2, 48.5, 41.9, 40.1, 36.2, 32.9, 31.4, 31.1, 29.7, 29.5, 29.4, 27.6, 25.8, 19.0, 14.3, 13.2. Peak overlapping was observed. HRMS (ESI) scaled for $[\mathrm{M}+\mathrm{H}]^{+}$: $\mathrm{C}_{27} \mathrm{H}_{43} \mathrm{~N}_{2} \mathrm{O}_{2}, \mathrm{~m} / \mathrm{z}$ : 427.3319, observed: 427.3317.

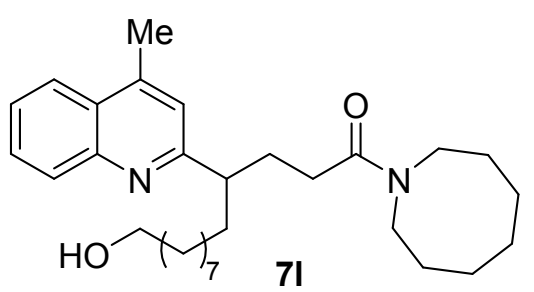

1-(Azocan-1-yl)-13-hydroxy-4-(4-methylquinolin-

2-yl)tridecan-1-one. Colorless oil (59.9 mg, 64\% yield), 0.2 mmol scale reaction. ${ }^{1} \mathbf{H}$ NMR $(500 \mathrm{MHz}$, chloroform- $d$ ) $\delta 8.03$ (dd, $J=8.4,1.2 \mathrm{~Hz}, 1 \mathrm{H}), 7.95$ $(\mathrm{dd}, J=8.4,1.4 \mathrm{~Hz}, 1 \mathrm{H}), 7.66(\mathrm{ddd}, J=8.4,6.8,1.4$ $\mathrm{Hz}, 1 \mathrm{H}), 7.50(\mathrm{ddd}, J=8.2,6.8,1.2 \mathrm{~Hz}, 1 \mathrm{H}), 7.14$ (s, $1 \mathrm{H}), 3.60(\mathrm{t}, J=6.7 \mathrm{~Hz}, 2 \mathrm{H}), 3.46-3.40(\mathrm{~m}, 1 \mathrm{H}), 3.34-3.29(\mathrm{~m}, 1 \mathrm{H}), 3.25-3.21(\mathrm{~m}$, $1 \mathrm{H}), 3.19-3.13(\mathrm{~m}, 1 \mathrm{H}), 2.92(\mathrm{tt}, J=8.5,5.5 \mathrm{~Hz}, 1 \mathrm{H}), 2.68(\mathrm{~s}, 3 \mathrm{H}), 2.19-2.08$ (comp, 2H), 1.82 - 1.64 (comp, 6H), 1.57 - 1.48 (comp, 3H), $1.48-1.39$ (comp, 7H), $1.34-$ 1.14 (comp, 12H). ${ }^{13} \mathbf{C}$ NMR (126 MHz, chloroform- $d$ ) $\delta 172.9,165.3,147.6,144.5$, 129.6, 129.1, 127.2, 125.6, 123.8, 121.0, 63.1, 49.1, 48.4, 47.4, 36.2, 32.9, 31.8, 30.9, 29.7, 29.5, 29.4, 27.6, 27.5, 27.1, 26.3, 25.8, 25.7, 25.3, 19.0. Peak overlapping was observed. HRMS (ESI) scaled for $[\mathrm{M}+\mathrm{H}]^{+}: \mathrm{C}_{30} \mathrm{H}_{47} \mathrm{~N}_{2} \mathrm{O}_{2}, \mathrm{~m} / \mathrm{z}: 467.3632$, observed: 467.3628.

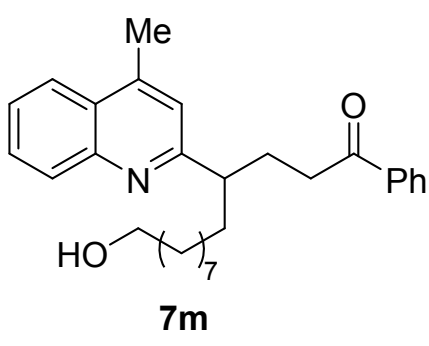

13-Hydroxy-4-(4-methylquinolin-2-yl)-1-

phenyltridecan-1-one. Colorless oil (41.1 mg, 48\% yield), 0.2 mmol scale reaction. ${ }^{1} \mathbf{H}$ NMR $(500 \mathrm{MHz}$, chloroformd) $\delta 8.06(\mathrm{dd}, J=8.4,1.2 \mathrm{~Hz}, 1 \mathrm{H}), 7.96(\mathrm{dd}, J=8.4,1.4$ $\mathrm{Hz}, 1 \mathrm{H}$ ), $7.87-7.79$ (comp, 2H), 7.68 (ddd, $J=8.4,6.8$, $1.4 \mathrm{~Hz}, 1 \mathrm{H}), 7.56-7.45$ (comp, 2H), 7.38 - 7.35 (comp, $2 \mathrm{H}), 7.13(\mathrm{~s}, 1 \mathrm{H}), 3.61(\mathrm{t}, J=6.7 \mathrm{~Hz}, 2 \mathrm{H}), 3.03-2.87$ (comp, 2H), $2.80-2.73(\mathrm{~m}, 1 \mathrm{H}), 2.68(\mathrm{~s}, 3 \mathrm{H}), 2.25-2.20$ (comp, 2H), $1.86-1.71$ (comp, 3H), 1.56 - 1.49 (comp, 2H), 1.35 - 1.20 (comp, 11H). ${ }^{13}$ C NMR (126 MHz, chloroform- $d$ ) $\delta 200.6,165.0,147.8,144.6,137.0,133.0,129.7,129.1,128.6,128.2$, 127.3, 125.7, 123.8, 121.0, 63.2, 48.4, 37.0, 36.2, 32.9, 30.0, 29.8, 29.6, 29.5, 27.7, 25.8, 19.0. Peak overlapping was observed. HRMS (ESI) scaled for $[\mathrm{M}+\mathrm{H}]^{+}: \mathrm{C}_{29} \mathrm{H}_{38} \mathrm{NO}_{2}$, m/z: 432.2897, observed: 432.2897 .

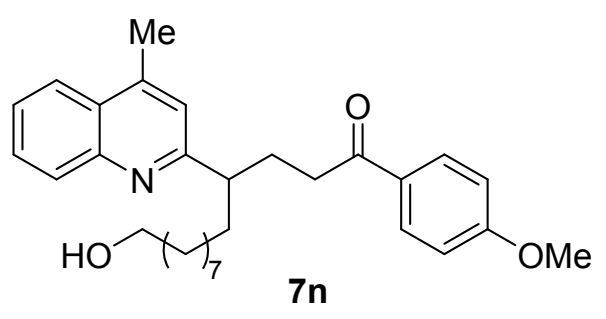

13-Hydroxy-1-(4-methoxyphenyl)-4-(4methylquinolin-2-yl)tridecan-1-one. Colorless oil (40.4 mg, $44 \%$ yield), $0.2 \mathrm{mmol}$ scale reaction. ${ }^{1}$ H NMR (500 MHz, chloroform- $d$ ) $\delta 8.09$ (dd, $J$ $=8.2 \mathrm{~Hz}, 1.3 \mathrm{~Hz}, 1 \mathrm{H}), 7.98(\mathrm{dd}, J=8.2,1.3 \mathrm{~Hz}$, $1 \mathrm{H}), 7.84$ (d, $J=8.8 \mathrm{~Hz}, 2 \mathrm{H}), 7.71$ (ddd, $J=8.2$, $6.8,1.3 \mathrm{~Hz}, 1 \mathrm{H}), 7.54(\mathrm{ddd}, J=8.2,6.8,1.3 \mathrm{~Hz}$, 1H), $7.15(\mathrm{~s}, 1 \mathrm{H}), 6.93-6.81(\mathrm{comp}, 2 \mathrm{H}), 3.85(\mathrm{~s}, 3 \mathrm{H}), 3.63(\mathrm{t}, J=6.7 \mathrm{~Hz}, 2 \mathrm{H}), 3.02-$ 
$2.94(\mathrm{~m}, 1 \mathrm{H}), 2.92-2.86(\mathrm{~m}, 1 \mathrm{H}), 2.75(\mathrm{t}, J=7.8 \mathrm{~Hz}, 1 \mathrm{H}), 2.70(\mathrm{~s}, 3 \mathrm{H}), 2.26-2.21$ (comp, 2H), $1.90-1.71$ (comp, 2H), $1.59-1.50$ (comp, 2H), $1.41-1.18$ (comp, 12H). ${ }^{13}$ C NMR (126 MHz, chloroform- $d$ ) $\delta$ 199.3, 165.1, 163.4, 147.8, 144.5, 130.5, 130.1, $129.7,129.1,127.3,125.7,123.8,121.0,113.7,63.2,55.5,48.4,36.6,36.2,32.9,30.2$, 29.8, 29.6, 29.4, 27.7, 25.8, 19.0. Peak overlapping was observed. HRMS (ESI) scaled for $[\mathrm{M}+\mathrm{H}]^{+}: \mathrm{C}_{30} \mathrm{H}_{40} \mathrm{NO}_{3}, \mathrm{~m} / \mathrm{z}: 462.3003$, observed: 462.3003 .<smiles>COC(=O)C(CC(C)CO)CC(CC(C)=O)c1cc(C)c2ccccc2n1</smiles>

Dimethyl 2-(11-hydroxy-2-(4-methylquinolin-2-yl)undecyl)malonate. Colorless oil (48.9 mg, 55\% yield), 0.2 mmol scale reaction. ${ }^{1} \mathbf{H}$ NMR $(500 \mathrm{MHz}$, chloroform- $d$ ) $\delta 8.03(\mathrm{dd}, J=8.4,1.2 \mathrm{~Hz}, 1 \mathrm{H}), 7.95(\mathrm{dd}, J=8.3,1.4 \mathrm{~Hz}$, $1 \mathrm{H}), 7.66(\mathrm{ddd}, J=8.4,6.8,1.4 \mathrm{~Hz}, 1 \mathrm{H}), 7.50(\mathrm{ddd}, J=$ $8.3,6.8,1.2 \mathrm{~Hz}, 1 \mathrm{H}), 7.06(\mathrm{~s}, 1 \mathrm{H}), 3.87-3.81(\mathrm{~m}, 1 \mathrm{H})$, $3.80-3.77(\mathrm{~m}, 1 \mathrm{H}), 3.71(\mathrm{~s}, 3 \mathrm{H}), 3.64-3.58$ (comp, $2 \mathrm{H})$, 3.54 (s, 3H), 3.25 (dd, $J=9.2,5.8 \mathrm{~Hz}, 1 \mathrm{H}), 2.89$ (ddt, $J=9.7,8.5,5.6 \mathrm{~Hz}, 1 \mathrm{H}), 2.68$ (s, $3 \mathrm{H}), 2.47-2.34$ (comp, 2H), $1.85-1.76(\mathrm{~m}, 1 \mathrm{H}), 1.74-1.67$ (m, 1H), $1.55-1.48$ (comp, 2H), 1.35 - 1.23 (comp, 10H). ${ }^{13} \mathbf{C ~ N M R ~ ( 1 2 6 ~ M H z , ~ c h l o r o f o r m - ~} d$ ) $\delta$ 170.0, 163.6, 147.9, 144.5, 129.8, 129.1, 127.2, 125.8, 123.7, 121.5, 63.1, 52.6, 52.5, 50.0, 46.3, 35.9, 34.0, 32.9, 29.7, 29.5, 29.4, 27.5, 25.82, 25.77, 19.0. HRMS (ESI) scaled for $[\mathrm{M}+\mathrm{H}]^{+}: \mathrm{C}_{26} \mathrm{H}_{38} \mathrm{NO}_{5}, \mathrm{~m} / \mathrm{z}: 444.2744$, observed: 444.2744.

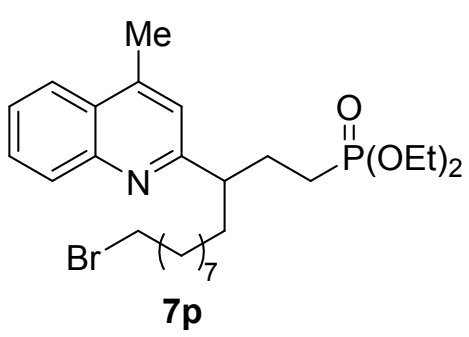

Diethyl [12-bromo-3-(4-methylquinolin-2-yl)dodecyllphosphonate. Colorless oil (56.0 mg, 53\% yield), $0.2 \mathrm{mmol}$ scale reaction. ${ }^{1} \mathbf{H}$ NMR $(500 \mathrm{MHz}$, chloroform- $d$ ) $\delta 8.02(\mathrm{dd}, J=8.5,1.2 \mathrm{~Hz}, 1 \mathrm{H}), 7.95$ (dd, $J=8.3,1.4 \mathrm{~Hz}, 1 \mathrm{H}), 7.66(\mathrm{ddd}, J=8.5,6.8,1.4 \mathrm{~Hz}, 1 \mathrm{H})$, 7.50 (ddd, $J=8.3,6.8,1.2 \mathrm{~Hz}, 1 \mathrm{H}), 7.08(\mathrm{~s}, 1 \mathrm{H}), 4.12-$ 3.94 (comp, 4H), 3.36 (t, $J=6.9 \mathrm{~Hz}, 2 \mathrm{H}), 2.89$ (tt, $J=$ 8.9, $5.7 \mathrm{~Hz}, 1 \mathrm{H}), 2.68$ (s, 3H), $2.15-1.97$ (comp, 3H), $1.85-1.74$ (comp, 3H), $1.72-$ 1.65 (comp, 2H), 1.55 - 1.45 (m, 1H), $1.39-1.30$ (comp, 2H), 1.30 - 1.15 (comp, 15H). ${ }^{13}$ C NMR (126 MHz, chloroform-d) $\delta$ 164.2, 147.8, 144.5, 129.7, 129.1, 127.2, $125.7,123.7,121.0,61.5\left(\mathrm{~d},{ }^{2} J_{\mathrm{CP}}=6.3 \mathrm{~Hz}\right), 61.4\left(\mathrm{~d},{ }^{2} J_{\mathrm{CP}}=6.2 \mathrm{~Hz}\right), 49.3(\mathrm{~d}, J=17.2$ $\mathrm{Hz}), 35.6,34.1,32.9,29.4,29.2\left(\mathrm{~d},{ }^{1} J_{\mathrm{CP}}=118.2 \mathrm{~Hz}\right), 28.2,28.0(\mathrm{~d}, J=4.6 \mathrm{~Hz}), 27.6$, 24.5, 23.3, 19.0, 16.6, 16.5. HRMS (ESI) scaled for $[\mathrm{M}+\mathrm{H}]^{+}: \mathrm{C}_{26} \mathrm{H}_{42} \mathrm{BrNO}_{3} \mathrm{P}, \mathrm{m} / \mathrm{z}$ : 526.2080, observed: 526.2063 .

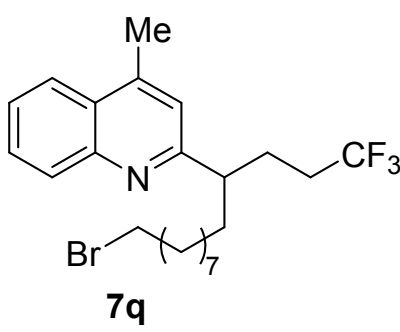

\section{2-(13-Bromo-1,1,1-trifluorotridecan-4-yl)-4-}

methylquinoline. Colorless oil (59.9 mg, 65\% yield), 0.2 mmol scale reaction. ${ }^{1} \mathbf{H}$ NMR $(500 \mathrm{MHz}$, chloroform- $d$ ) $\delta$ $8.05(\mathrm{dd}, J=8.4,1.3 \mathrm{~Hz}, 1 \mathrm{H}), 7.97(\mathrm{dd}, J=8.3,1.4 \mathrm{~Hz}, 1 \mathrm{H})$, 7.69 (ddd, $J=8.4,6.8,1.4 \mathrm{~Hz}, 1 \mathrm{H}), 7.53(\mathrm{ddd}, J=8.2,6.8$, $1.3 \mathrm{~Hz}, 1 \mathrm{H}), 7.09$ (s, 1H), 3.37 (t, $J=6.9 \mathrm{~Hz}, 2 \mathrm{H}), 2.90$ (tt, $J$ $=8.9,5.4 \mathrm{~Hz}, 1 \mathrm{H}), 2.70(\mathrm{~s}, 3 \mathrm{H}), 2.14-1.95(\mathrm{comp}, 3 \mathrm{H}), 1.87$

- 1.77 (comp, 4H), 1.73 - 1.66 (m, 1H), 1.43 - 1.33 (comp, 2H), 1.31 - 1.18 (comp, 9H), $1.16-1.11(\mathrm{~m}, 1 \mathrm{H}) .{ }^{13} \mathrm{C}$ NMR (126 MHz, chloroform- $d$ ) $\delta 163.9,147.9,144.7$, $129.8,129.2,127.3,127.40\left(\mathrm{q},{ }^{1} J_{\mathrm{CF}}=276.4 \mathrm{~Hz}\right), 125.9,123.8,120.9,77.4,47.7,35.8$, $34.2,32.9,32.2\left(\mathrm{q},{ }^{2} J_{\mathrm{CF}}=28.5 \mathrm{~Hz}\right), 29.7,29.4,28.8,28.3,27.6,27.4$, 19.0. Peak 
overlapping was observed. HRMS (ESI) scaled for $[\mathrm{M}+\mathrm{H}]^{+}: \mathrm{C}_{23} \mathrm{H}_{32} \mathrm{BrF}{ }_{3} \mathrm{~N}, \mathrm{~m} / \mathrm{z}$ : 458.1665, observed: 458.1649 .<smiles>CCOC(=O)CCC(CCBr)c1cc(-c2ccccc2)c2ccccc2n1</smiles>

Ethyl 13-bromo-4-(4-phenylquinolin-2-yl)tridecanoate. Colorless oil (79.9 mg, 76\% yield), $0.2 \mathrm{mmol}$ scale reaction. ${ }^{1} \mathbf{H}$ NMR $(500 \mathrm{MHz}$, chloroform- $d$ ) $\delta 8.12(\mathrm{dd}, J$ $=8.5,1.2 \mathrm{~Hz}, 1 \mathrm{H}), 7.89(\mathrm{dd}, J=8.3,1.4 \mathrm{~Hz}, 1 \mathrm{H}), 7.69$ (ddd, $J=8.5,6.8,1.4 \mathrm{~Hz}, 1 \mathrm{H}$ ), $7.57-7.45$ (comp, 5H), 7.45 (ddd, $J=8.3,6.8,1.2 \mathrm{~Hz}, 1 \mathrm{H}), 7.20(\mathrm{~s}, 1 \mathrm{H}), 4.09-$ 3.99 (comp, 2H), 3.37 (t, $J=6.9 \mathrm{~Hz}, 2 \mathrm{H}), 3.01-2.95(\mathrm{~m}, 1 \mathrm{H}), 2.33-2.27(\mathrm{~m}, 1 \mathrm{H})$, $2.23-2.12$ (comp, 3H), $1.89-1.72$ (comp, 5H), $1.41-1.19$ (comp, 11H), 1.18 (t, $J=$ $7.1 \mathrm{~Hz}, 3 \mathrm{H}) .{ }^{13} \mathrm{C}$ NMR $(126 \mathrm{MHz}$, chloroform- $d$ ) $\delta 173.7,164.5,148.7,148.5,138.4$, $129.7,129.7,129.3,128.7,128.5,126.0,125.7,125.7,120.7,60.3,48.3,35.7,34.1$, 32.9, 32.7, 30.6, 29.7, 29.4, 28.8, 28.2, 27.6, 14.3. Peak overlapping was observed. HRMS (ESI) scaled for $[\mathrm{M}+\mathrm{H}]^{+}: \mathrm{C}_{30} \mathrm{H}_{39} \mathrm{BrNO}_{2}, \mathrm{~m} / \mathrm{z}: 524.2159$, observed: 524.2148.<smiles>CCOC(CC)CCC(CCBr)C(CCBr)c1cc(C(C)C)c2ccccc2n1</smiles>

Ethyl 13-bromo-4-(4-isopropylquinolin-2-yl)tridecanoate. Colorless oil ( $80.1 \mathrm{mg}, 82 \%$ yield), $0.2 \mathrm{mmol}$ scale reaction. ${ }^{1} \mathbf{H}$ NMR $(500 \mathrm{MHz}$, chloroform- $d$ ) $\delta 8.06(\mathrm{dd}, J$ $=8.3,1.3 \mathrm{~Hz}, 1 \mathrm{H}), 8.04(\mathrm{dd}, J=8.4,1.3 \mathrm{~Hz}, 1 \mathrm{H}), 7.65$ (ddd, $J=8.3,6.8,1.3 \mathrm{~Hz}, 1 \mathrm{H}), 7.49$ (ddd, $J=8.4,6.8,1.3$ $\mathrm{Hz}, 1 \mathrm{H}), 7.14$ (s, 1H), $4.09-4.00$ (comp, 2H), 3.71 (hept, $J=6.9 \mathrm{~Hz}, 1 \mathrm{H}), 3.36(\mathrm{t}, J=6.9 \mathrm{~Hz}, 2 \mathrm{H}), 2.97-2.85(\mathrm{~m}, 1 \mathrm{H}), 2.30-2.19(\mathrm{~m}, 1 \mathrm{H}), 2.18$ - 2.06 (comp, 3H), $1.88-1.76$ (comp, 3H), $1.77-1.68$ (m, 1H), 1.40 (d, $J=6.9 \mathrm{~Hz}$, $6 \mathrm{H}), 1.31-1.14$ (comp, 15H). ${ }^{13} \mathbf{C}$ NMR (126 MHz, chloroform-d) $\delta$ 173.8, 164.7, 154.4, 148.3, 130.2, 128.7, 125.8, 125.5, 123.0, 116.2, 60.3, 48.3, 35.6, 34.1, 32.9, 32.6, 30.5, 29.7, 29.4, 28.8, 28.5, 28.2, 27.6, 23.1, 14.3. Peak overlapping was observed. HRMS (ESI) scaled for $[\mathrm{M}+\mathrm{H}]^{+}: \mathrm{C}_{27} \mathrm{H}_{41} \mathrm{BrNO}_{2}, \mathrm{~m} / \mathrm{z}: 490.2315$, observed: 490.2294.<smiles>CCOC(=O)CCC(CCBr)c1cc(Cl)c2ccccc2n1</smiles>

Ethyl 13-bromo-4-(4-cyclohexylquinolin-2-yl)tridecanoate. Colorless oil $(91.2 \mathrm{mg}, 86 \%$ yield $), 0.2 \mathrm{mmol}$ scale reaction. ${ }^{1} \mathbf{H}$ NMR $(500 \mathrm{MHz}$, chloroform- $d) \delta 8.06$ - 8.02 (comp, 2H), 7.64 (ddd, $J=8.3,6.7,1.3 \mathrm{~Hz}, 1 \mathrm{H})$, 7.48 (ddd, $J=8.1,6.7,1.3 \mathrm{~Hz}, 1 \mathrm{H}), 7.12(\mathrm{~s}, 1 \mathrm{H}), 4.09-$ 3.99 (comp, 2H), $3.36(\mathrm{t}, J=6.9 \mathrm{~Hz}, 2 \mathrm{H}), 3.32-3.27(\mathrm{~m}$, 1H), $2.91-2.87$ (m, 1H), $2.27-2.19$ (m, 1H), $2.17-2.07$ (comp, 3H), $2.06-1.90$ (comp, 4H), 1.87 - 1.68 (comp, 5H), $1.60-1.49$ (comp, 4H), 1.39 - 1.32 (comp, 3H), 1.26 - 1.11 (comp, 13H). ${ }^{13} \mathbf{C}$ NMR (126 MHz, chloroform- $d$ ) $\delta$ 173.8, 164.7, 153.5, $148.3,130.2$, 128.7, 125.8, 125.4, 122.9, 116.7, 60.3, 48.3, 39.0, 35.6, 34.1, 33.7, 32.9, 32.6, 30.5, 29.68, 29.67, 29.4, 28.8, 28.2, 27.6, 27.0, 26.4, 14.3. HRMS (ESI) scaled for $[\mathrm{M}+\mathrm{H}]^{+}: \mathrm{C}_{30} \mathrm{H}_{45} \mathrm{BrNO}_{2}, \mathrm{~m} / \mathrm{z}: 530.2628$, observed: 530.2612.<smiles>CCOC(=O)CCC(CCCCBr)c1cc(Cl)c2ccc(OC)cc2n1</smiles>

Ethyl 13-bromo-4-(4-chloro-7-methoxyquinolin2-yl)tridecanoate. Colorless oil (44.3 $\mathrm{mg}, 43 \%$ yield), $0.2 \mathrm{mmol}$ scale reaction. ${ }^{1} \mathbf{H}$ NMR $(500 \mathrm{MHz}$, chloroform- $d) \delta 8.05(\mathrm{~d}, J=9.2 \mathrm{~Hz}, 1 \mathrm{H}), 7.37(\mathrm{~d}, J$ $=2.6 \mathrm{~Hz}, 1 \mathrm{H}), 7.22(\mathrm{dd}, J=9.2,2.6 \mathrm{~Hz}, 1 \mathrm{H}), 7.19$ (s, 1H), $4.09-4.01$ (comp, 2H), 3.95 (s, 3H), 3.37 
(t, $J=6.9 \mathrm{~Hz}, 2 \mathrm{H}), 2.86(\mathrm{tt}, J=8.8,5.7 \mathrm{~Hz}, 1 \mathrm{H}), 2.26-2.20(\mathrm{~m}, 1 \mathrm{H}), 2.16-2.11(\mathrm{~m}$, 1H), 2.12 - 1.99 (comp, 2H), 1.86 - 1.76 (comp, 3H), 1.75 - 1.64 (comp, 2H), 1.39 1.32 (comp, 3H), 1.30 - 1.23 (comp, 5H), $1.22-1.17$ (comp, 6H). ${ }^{13} \mathbf{C}$ NMR (126 MHz, chloroform- $d$ ) $\delta 173.6,165.6,161.5,150.7,142.6,125.2,120.3,120.0,118.4,107.6$, $60.4,55.8,48.2,35.7,34.1,32.9,32.5,30.5,29.7,29.4,28.8,28.3,27.6,14.3$. Peak overlapping was observed. HRMS (ESI) scaled for $[\mathrm{M}+\mathrm{H}]^{+}: \mathrm{C}_{25} \mathrm{H}_{36} \mathrm{BrClNO}_{3}, \mathrm{~m} / \mathrm{z}$ : 512.1562, observed: 512.1562 .

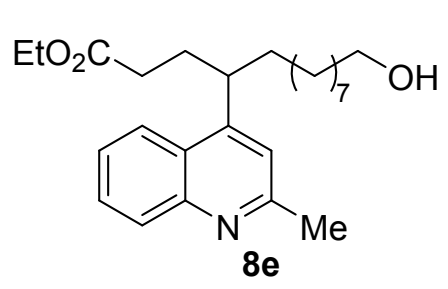

Ethyl 13-hydroxy-4-(2-methylquinolin-4-yl)tridecanoate. Colorless oil $(64.7 \mathrm{mg}, 81 \%$ yield), $0.2 \mathrm{mmol}$ scale reaction. ${ }^{1} \mathbf{H}$ NMR $(500 \mathrm{MHz}$, chloroform- $d$ ) $\delta 8.06-8.01$ (comp, 2H), 7.66 (ddd, $J=8.2,6.8,1.3 \mathrm{~Hz}, 1 \mathrm{H}), 7.48$ (ddd, $J=8.3,6.8,1.3 \mathrm{~Hz}, 1 \mathrm{H}), 7.15(\mathrm{~s}, 1 \mathrm{H}), 4.09-3.99$ (comp, 2H), $3.60(\mathrm{t}, J=6.7 \mathrm{~Hz}, 2 \mathrm{H}), 2.73(\mathrm{~s}, 3 \mathrm{H}), 2.25-2.09$ (comp, 3H), $2.04-2.00$ (m, 1H), $1.86-1.67$ (comp, 3H), $1.57-1.45$ (comp, 2H), 1.31 - 1.20 (comp, 10H), $1.19-1.15$ (comp, 5H). ${ }^{13} \mathbf{C}$ NMR (126 MHz, chloroform- $d$ ) $\delta$ $173.5,158.7,151.6,148.4,129.7,129.2,126.5,125.6,122.9,119.1,63.1,60.5,36.3$, 32.9, 32.2 , 31.1 29.71, 29.69, 29.5, 29.40, 29.37, 27.5, 25.8, 25.6, 14.3. HRMS (ESI) scaled for $[\mathrm{M}+\mathrm{H}]^{+}: \mathrm{C}_{25} \mathrm{H}_{38} \mathrm{NO}_{3}, \mathrm{~m} / \mathrm{z}$ : 400.2846, observed: 400.2846 .

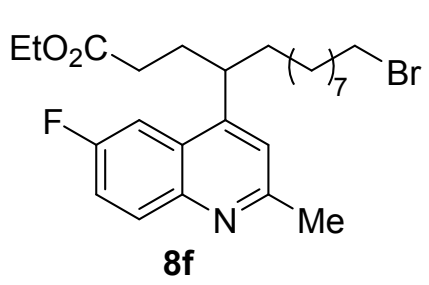

Ethyl 13-bromo-4-(6-fluoro-2-methylquinolin-4-yl)tridecanoate. Colorless oil ( $78.6 \mathrm{mg}, 82 \%$ yield), $0.2 \mathrm{mmol}$ scale reaction. ${ }^{1} \mathbf{H}$ NMR $(500 \mathrm{MHz}$, chloroform- $d$ ) $\delta 8.01$ (dd, $J=9.2,5.7 \mathrm{~Hz}, 1 \mathrm{H}), 7.61(\mathrm{dd}, J=10.6,2.8 \mathrm{~Hz}, 1 \mathrm{H})$, 7.42 (ddd, $J=9.2,7.9,2.8 \mathrm{~Hz}, 1 \mathrm{H}), 7.15(\mathrm{~s}, 1 \mathrm{H}), 4.12-$ $3.98(\mathrm{comp}, 2 \mathrm{H}), 3.36(\mathrm{t}, J=6.8 \mathrm{~Hz}, 2 \mathrm{H}), 2.70(\mathrm{~s}, 3 \mathrm{H}), 2.18$ -2.08 (comp, 3H), $2.01-1.95$ (m, 1H), $1.82-1.75$ (comp, 3H), $1.71-1.69$ (m, 1H), $1.40-1.31$ (comp, 3H), $1.25-1.13$ (comp, 13H). ${ }^{13}$ C NMR (126 MHz, chloroform- $d$ ) $\delta 173.3,160.3\left(\mathrm{~d},{ }^{1} J_{\mathrm{CF}}=245.4 \mathrm{~Hz}\right), 158.0,151.0,145.5,132.0\left(\mathrm{~d},{ }^{3} J_{\mathrm{CF}}=9.0 \mathrm{~Hz}\right), 127.1$ $\left(\mathrm{d},{ }^{3} J_{\mathrm{CF}}=7.9 \mathrm{~Hz}\right), 120.0,119.1\left(\mathrm{~d},{ }^{2} J_{\mathrm{CF}}=25.4 \mathrm{~Hz}\right), 106.67\left(\mathrm{~d},{ }^{2} J_{\mathrm{CF}}=23.1 \mathrm{~Hz}\right), 60.5$, $36.1,34.1,32.9,32.1,31.0,29.7,29.4,29.3,28.7,28.2,27.4,25.4,14.2$. Peak overlapping was observed. HRMS (ESI) scaled for $[\mathrm{M}+\mathrm{H}]^{+}: \mathrm{C}_{25} \mathrm{H}_{36} \mathrm{BrFNO}_{2}, \mathrm{~m} / \mathrm{z}$ : 480.1908, observed: 480.1908.

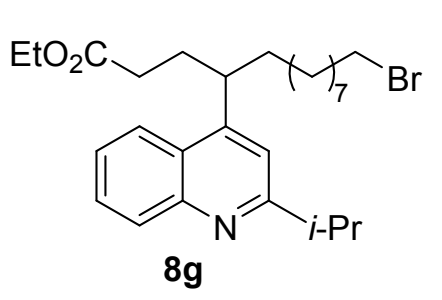

Ethyl

13-bromo-4-(2-isopropylquinolin-4-

yl)tridecanoate. Colorless oil $(81.2 \mathrm{mg}, 83 \%$ yield $), 0.2$ mmol scale reaction. ${ }^{1} \mathbf{H}$ NMR $(500 \mathrm{MHz}$, chloroform- $d$ ) $\delta$ $8.06(\mathrm{dd}, J=8.4,1.3 \mathrm{~Hz}, 1 \mathrm{H}), 8.02(\mathrm{dd}, J=8.4,1.3 \mathrm{~Hz}$, $1 \mathrm{H}), 7.65$ (ddd, $J=8.4,6.8,1.3 \mathrm{~Hz}, 1 \mathrm{H}), 7.47$ (ddd, $J=8.4$, $6.8,1.3 \mathrm{~Hz}, 1 \mathrm{H}), 7.18$ (s, 1H), $4.07-3.99$ (comp, 2H), 3.60 $-3.44(\mathrm{~m}, 1 \mathrm{H}), 3.36(\mathrm{t}, J=6.9 \mathrm{~Hz}, 2 \mathrm{H}), 3.23$ (hept, $J=6.9 \mathrm{~Hz}, 1 \mathrm{H}), 2.21-2.10$ (comp, $3 \mathrm{H}), 2.06-2.01(\mathrm{~m}, 1 \mathrm{H}), 1.82-1.75$ (comp, $4 \mathrm{H}), 1.41-1.32$ (comp, 9H), $1.27-1.09$ (comp, 12H). ${ }^{13} \mathbf{C}$ NMR (126 MHz, chloroform- $d$ ) $\delta 173.5,167.3,151.4,148.3,130.1$, $128.9,126.8,125.5,122.8,116.2,60.4,37.4,36.1,34.1,32.9,32.2,31.0,29.7,29.4$, 29.3, 28.7, 28.2, 27.4, 22.7, 22.6, 14.3. HRMS (ESI) scaled for $[\mathrm{M}+\mathrm{H}]^{+}: \mathrm{C}_{27} \mathrm{H}_{41} \mathrm{BrNO}_{2}$, $\mathrm{m} / \mathrm{z}: 490.2315$, observed: 490.2302 . 


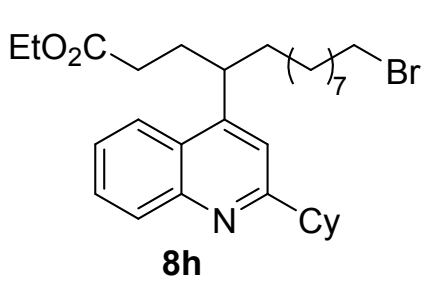

Ethyl 13-bromo-4-(2-cyclohexylquinolin-4-yl)tridecanoate. Colorless oil (78.3 mg, $74 \%$ yield), $0.2 \mathrm{mmol}$ scale reaction. ${ }^{1} \mathbf{H}$ NMR $(500 \mathrm{MHz}$, chloroform- $d$ ) $\delta 8.06(\mathrm{dd}, J$ $=8.4,1.3 \mathrm{~Hz}, 1 \mathrm{H}), 8.02(\mathrm{dd}, J=8.3,1.3 \mathrm{~Hz}, 1 \mathrm{H}), 7.65(\mathrm{ddd}$, $J=8.4,6.8,1.3 \mathrm{~Hz}, 1 \mathrm{H}), 7.47(\mathrm{ddd}, J=8.3,6.8,1.3 \mathrm{~Hz}$, 1H), 7.17 (s, 1H), $4.12-3.97$ (comp, 2H), $3.53-3.48$ (m, $1 \mathrm{H}), 3.37$ (t, $J=6.9 \mathrm{~Hz}, 2 \mathrm{H}), 2.91-2.85(\mathrm{~m}, 1 \mathrm{H}), 2.19-2.11$ (comp, 2H), $2.07-1.95$ (comp, 3H), $1.94-1.88$ (comp, 2H), $1.84-1.72$ (comp, 5H), $1.68-1.57$ (comp, 2H), $1.52-1.43$ (comp, 2H), $1.40-1.33$ (comp, 3H), $1.27-1.11$ (comp, 14H). ${ }^{13}$ C NMR $(126 \mathrm{MHz}$, chloroform- $d$ ) $\delta 173.5,166.5,151.3,148.3,130.1,128.9,126.9,125.5$, $122.8,116.6,60.4,47.7,36.1,34.1,33.0,32.93,32.88,32.1,31.0,29.7,29.4,29.3,28.7$, 28.2, 27.4, 26.7, 26.2, 14.2. Peak overlapping was observed. HRMS (ESI) scaled for $[\mathrm{M}+\mathrm{H}]^{+}: \mathrm{C}_{30} \mathrm{H}_{45} \mathrm{BrNO}_{2}, \mathrm{~m} / \mathrm{z}$ : 530.2628, observed: 530.2614.<smiles>CCOC(=O)CCC(CCBr)c1ccc2ccccc2n1</smiles>

\section{Ethyl}

13-bromo-4-(quinolin-2-yl)tridecanoate. Colorless oil (37.6 mg, 42\% yield), $0.2 \mathrm{mmol}$ scale reaction. ${ }^{1} \mathbf{H}$ NMR $(500 \mathrm{MHz}$, chloroform- $d$ ) $\delta 8.08(\mathrm{~d}, J$ $=8.4 \mathrm{~Hz}, 1 \mathrm{H}), 8.05(\mathrm{dd}, J=8.5,1.1 \mathrm{~Hz}, 1 \mathrm{H}), 7.78(\mathrm{dd}, J$ $=8.1,1.4 \mathrm{~Hz}, 1 \mathrm{H}), 7.68(\mathrm{ddd}, J=8.5,6.8,1.4 \mathrm{~Hz}, 1 \mathrm{H})$, 7.49 (ddd, $J=8.1,6.8,1.1 \mathrm{~Hz}, 1 \mathrm{H}), 7.27$ (d, $J=8.4 \mathrm{~Hz}, 1 \mathrm{H}), 4.08-3.99$ (comp, 2H), 3.37 (t, $J=6.9 \mathrm{~Hz}, 2 \mathrm{H}), 2.98-2.92(\mathrm{~m}, 1 \mathrm{H}), 2.27-2.20$ (m, 1H), $2.17-2.05$ (comp, $3 \mathrm{H}), 1.84-1.77$ (comp, 3H), $1.76-1.70$ (m, 1H), $1.43-1.32$ (comp, 2H), $1.28-1.14$ (comp, 13H). ${ }^{13} \mathbf{C}$ NMR (126 MHz, chloroform-d) $\delta 173.8,165.0,148.0,136.5,129.4$, 129.3, 127.6, 127.2, 125.9, 120.4, 60.4, 48.3, 35.8, 34.2, 32.9, 32.6, 30.6, 29.7, 29.4, 28.8, 28.3, 27.6, 14.3. Peak overlapping was observed. HRMS (ESI) scaled for $[\mathrm{M}+\mathrm{H}]^{+}$: $\mathrm{C}_{24} \mathrm{H}_{35} \mathrm{BrNO}_{2}, \mathrm{~m} / \mathrm{z}$ : 448.1846, observed: 448.1846 .

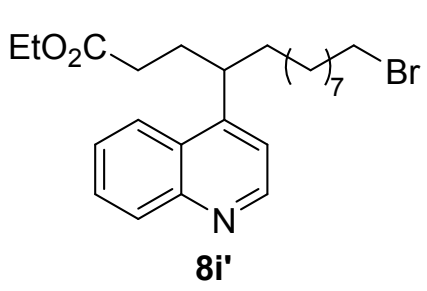

Ethyl 13-bromo-4-(quinolin-4-yl)tridecanoate. Colorless oil (27.5 mg, 31\% yield), $0.2 \mathrm{mmol}$ scale reaction. ${ }^{1} \mathbf{H}$ NMR (500 MHz, chloroform- $d$ ) $\delta 8.86(\mathrm{~d}, J=4.6 \mathrm{~Hz}, 1 \mathrm{H}), 8.13$ (dd, $J=8.4,1.3 \mathrm{~Hz}, 1 \mathrm{H}), 8.09$ (dd, $J=8.3,1.3 \mathrm{~Hz}, 1 \mathrm{H})$, 7.70 (ddd, $J=8.4,6.7,1.3 \mathrm{~Hz}, 1 \mathrm{H}), 7.55$ (ddd, $J=8.3,6.7$, $1.3 \mathrm{~Hz}, 1 \mathrm{H}), 7.27$ (d, $J=4.6 \mathrm{~Hz}, 1 \mathrm{H}), 4.08-3.99$ (comp, $2 \mathrm{H}), 3.61-3.52(\mathrm{~m}, 1 \mathrm{H}), 3.36(\mathrm{t}, J=6.9 \mathrm{~Hz}, 2 \mathrm{H}), 2.23-2.10$ (comp, $3 \mathrm{H}), 2.08-1.98$ (m, 1H), $1.82-1.76$ (comp, 4H), $1.38-1.30$ (comp, 3H), $1.23-1.11$ (comp, $12 \mathrm{H}) .{ }^{13} \mathbf{C}$ NMR (126 MHz, chloroform-d) $\delta$ 173.4, 151.6, 150.3, 148.6, 130.6, 129.2, 128.2, $126.5,123.1,118.3,60.5,36.3,34.1,32.9,32.1,31.1,29.7,29.38,29.36,28.8,28.2$, 27.5, 14.3. Peak overlapping was observed. HRMS (ESI) scaled for $[\mathrm{M}+\mathrm{H}]^{+}$: $\mathrm{C}_{24} \mathrm{H}_{35} \mathrm{BrNO}_{2}, \mathrm{~m} / \mathrm{z}$ : 448.1846, observed: 448.1846 .

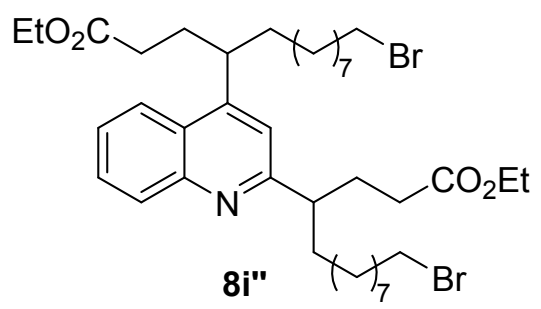

Diethyl 4,4'-(quinoline-2,4-diyl)bis(13-bromotridecanoate). Colorless oil (33.5 mg, $22 \%$ yield), 0.2 mmol scale reaction. ${ }^{\mathbf{H}} \mathbf{H}$ NMR $(500 \mathrm{MHz}$, chloroform- $d) \delta 8.06(\mathrm{~d}, J=8.3 \mathrm{~Hz}, 1 \mathrm{H}), 8.03(\mathrm{~d}, J=$ $8.4 \mathrm{~Hz}, 1 \mathrm{H}), 7.66(\mathrm{dd}, J=8.3,7.6 \mathrm{~Hz}, 1 \mathrm{H}), 7.48(\mathrm{dd}$, $J=8.4,7.6 \mathrm{~Hz}, 1 \mathrm{H}), 7.10(\mathrm{~s}, 1 \mathrm{H}), 4.07-4.02$ (comp, $4 \mathrm{H}), 3.61-3.48(\mathrm{~m}, 1 \mathrm{H}), 3.40-3.33$ (comp, $4 \mathrm{H}), 2.97-2.84(\mathrm{~m}, 1 \mathrm{H}), 2.27-2.06$ 
(comp, 7H), $2.06-1.98$ (m, 1H), $1.86-1.69$ (comp, 8H), $1.38-1.32$ (comp, 4H), 1.29 -1.09 (comp, 26H). ${ }^{13} \mathrm{C}$ NMR (126 MHz, chloroform- $d$ ) $\delta 173.7,173.4,164.5,151.3$, 148.5, 130.3, 129.0, 126.9, 125.7, 122.9, 117.5, 60.4, 60.3, 48.23, 48.17, 36.03, 35.99, 35.7, 35.6, 34.10, 34.09, 32.9, 32.6, 32.1, 31.04, 31.00, 30.6, 30.5, 29.7, 29.5, 29.4, 28.81, 28.77, 28.3, 28.2, 27.6, 27.4, 14.3, 14.3. HRMS (ESI) scaled for $[\mathrm{M}+\mathrm{H}]^{+}$: $\mathrm{C}_{39} \mathrm{H}_{62} \mathrm{Br}_{2} \mathrm{NO}_{4}, \mathrm{~m} / \mathrm{z}$ : 766.3040 , observed: 766.3035 .

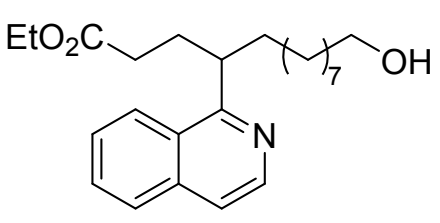

$8 \mathbf{j}$

Ethyl 13-hydroxy-4-(isoquinolin-1-yl)tridecanoate. Colorless oil (48.5 mg, 63\% yield), $0.2 \mathrm{mmol}$ scale reaction. ${ }^{1} \mathbf{H}$ NMR $(500 \mathrm{MHz}$, chloroform- $d$ ) $\delta 8.51(\mathrm{~d}, J$ $=5.6 \mathrm{~Hz}, 1 \mathrm{H}), 8.20(\mathrm{~d}, J=8.4 \mathrm{~Hz}, 1 \mathrm{H}), 7.82(\mathrm{dd}, J=8.2$, $1.2 \mathrm{~Hz}, 1 \mathrm{H}), 7.66(\mathrm{ddd}, J=8.2,6.8,1.2 \mathrm{~Hz}, 1 \mathrm{H}), 7.58$ (ddd, $J=8.4,6.8,1.2 \mathrm{~Hz}, 1 \mathrm{H}), 7.49(\mathrm{~d}, J=5.6 \mathrm{~Hz}, 1 \mathrm{H}), 4.04(\mathrm{q}$, $J=7.1 \mathrm{~Hz}, 2 \mathrm{H}), 3.81-3.72(\mathrm{~m}, 1 \mathrm{H}), 3.61(\mathrm{t}, J=6.7 \mathrm{~Hz}, 2 \mathrm{H}), 2.36-2.23(\mathrm{~m}, 1 \mathrm{H}), 2.21$ - 2.04 (comp, 3H), $1.98-1.91(\mathrm{~m}, 1 \mathrm{H}), 1.79-1.71(\mathrm{~m}, 1 \mathrm{H}), 1.57-1.46$ (comp, 2H), 1.34 - 1.11 (comp, 15H). ${ }^{13}$ C NMR (126 MHz, chloroform-d) $\delta 173.9,164.2,142.2$, 136.4, 129.8, 128.0, 127.7, 127.1, 124.8, 119.1, 63.2, 60.3, 40.5, 35.8, 32.9, 32.4, 30.3, 29.9, 29.6, 29.4, 27.7 25.8, 14.3. Peak overlapping was observed. HRMS (ESI) scaled for $[\mathrm{M}+\mathrm{H}]^{+}: \mathrm{C}_{24} \mathrm{H}_{36} \mathrm{NO}_{3}, \mathrm{~m} / \mathrm{z}$ : 386.2690, observed: 386.2690 .

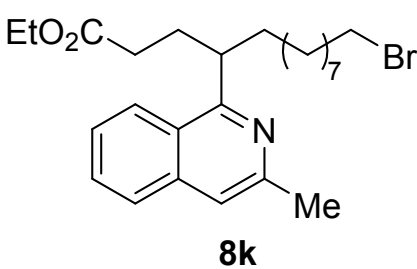

$3.37(\mathrm{t}, J=6.9 \mathrm{~Hz}, 2 \mathrm{H}), 2.65(\mathrm{~s}, 3 \mathrm{H}), 2.38-2.27(\mathrm{~m}, 1 \mathrm{H}), 2.18-2.09$ (comp, 3H), 1.99 - $1.92(\mathrm{~m}, 1 \mathrm{H}), 1.83-1.78$ (comp, 2H), $1.75-1.69$ (m, 1H), $1.40-1.32$ (comp, 2H), 1.26 - 1.10 (comp, 13H). ${ }^{13} \mathbf{C}$ NMR (126 MHz, chloroform-d) $\delta$ 174.0, 163.4, 150.9, 137.2 , 129.5, 127.0, 126.0, 125.9, 124.7, 116.9, 60.2, 40.7, 35.8, 34.2, 33.0, 32.5, 30.2, 29.8, 29.4, 28.8, 28.3 , 27.8, 24.6, 14.3. Peak overlapping was observed. HRMS (ESI) scaled for $[\mathrm{M}+\mathrm{H}]^{+}: \mathrm{C}_{25} \mathrm{H}_{37} \mathrm{BrNO}_{2}, \mathrm{~m} / \mathrm{z}$ : 462.2002, observed: 462.2002 .

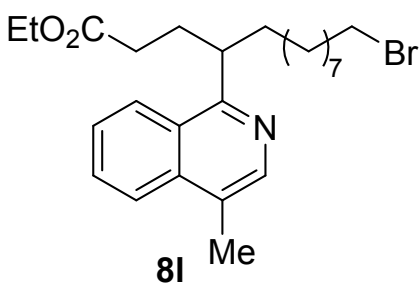

Ethyl 13-bromo-4-(4-methylisoquinolin-1-yl)tridecanoate. Colorless oil (56.6 mg, $61 \%$ yield), $0.2 \mathrm{mmol}$ scale reaction. ${ }^{1} \mathbf{H}$ NMR $(500 \mathrm{MHz}$, chloroform- $d$ ) $\delta 8.36(\mathrm{~s}, 1 \mathrm{H})$, 8.21 (dd, $J=8.4,1.2 \mathrm{~Hz}, 1 \mathrm{H}), 7.97$ (dd, $J=8.3,1.2 \mathrm{~Hz}$, $1 \mathrm{H}), 7.70(\mathrm{ddd}, J=8.3,6.8,1.2 \mathrm{~Hz}, 1 \mathrm{H}), 7.58(\mathrm{ddd}, J=8.4$, $6.8,1.2 \mathrm{~Hz}, 1 \mathrm{H}), 4.03(\mathrm{q}, J=7.1 \mathrm{~Hz}, 2 \mathrm{H}), 3.77-3.71(\mathrm{~m}$, $1 \mathrm{H}), 3.36(\mathrm{t}, J=6.9 \mathrm{~Hz}, 2 \mathrm{H}), 2.59(\mathrm{~s}, 3 \mathrm{H}), 2.33-2.22(\mathrm{~m}, 1 \mathrm{H}), 2.20-2.04(\mathrm{comp}, 3 \mathrm{H})$, $1.97-1.88$ (m, 1H), $1.84-1.67$ (comp, 4H), $1.36-1.32$ (comp, 2H), $1.25-1.11$ (m, 12H). ${ }^{13}$ C NMR (126 MHz, chloroform- $d$ ) $\delta$ 173.9, 162.4, 142.2, 135.8, 129.6, 127.4, 126.7, 125.2, 124.1, 119.1, 60.3, 40.2, 35.8, 34.2, 32.9, 32.4, 30.3, 29.8, 29.4, 28.8, 28.2, 27.7, 16.2, 14.3. Peak overlapping was observed. HRMS (ESI) scaled for $[\mathrm{M}+\mathrm{H}]^{+}$: $\mathrm{C}_{25} \mathrm{H}_{37} \mathrm{BrNO}_{2}$, m/z: 462.2002, observed: 462.1992. 


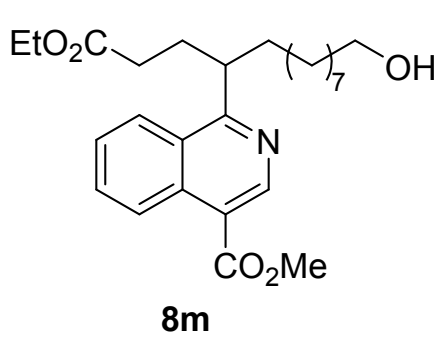

Methyl 1-(1-ethoxy-13-hydroxy-1-oxotridecan-4-yl)isoquinoline-4-carboxylate. Colorless oil $(33.1 \mathrm{mg}, 37 \%$ yield), $0.2 \mathrm{mmol}$ scale reaction. ${ }^{1} \mathbf{H}$ NMR $(500 \mathrm{MHz}$, chloroform- $d$ ) $\delta 9.15(\mathrm{~s}, 1 \mathrm{H}), 8.97(\mathrm{~d}, J=8.5 \mathrm{~Hz}, 1 \mathrm{H}), 8.28$ (d, $J=8.4 \mathrm{~Hz}, 1 \mathrm{H}), 7.80$ (ddd, $J=8.5,6.8,1.2 \mathrm{~Hz}, 1 \mathrm{H}$ ), 7.64 (ddd, $J=8.4,6.8,1.2 \mathrm{~Hz}, 1 \mathrm{H}), 4.04$ (q, $J=7.1 \mathrm{~Hz}$, $2 \mathrm{H}), 4.01(\mathrm{~s}, 3 \mathrm{H}), 3.86-3.61(\mathrm{~m}, 1 \mathrm{H}), 3.60(\mathrm{t}, J=6.6 \mathrm{~Hz}$, $2 \mathrm{H}), 2.37-2.25(\mathrm{~m}, 1 \mathrm{H}), 2.21-2.03(\mathrm{comp}, 3 \mathrm{H}), 1.99-1.88(\mathrm{~m}, 1 \mathrm{H}), 1.79-1.72(\mathrm{~m}$, 1H), $1.56-1.44$ (comp, 2H), $1.34-1.13$ (comp, 15H). ${ }^{13} \mathbf{C}$ NMR (126 MHz, chloroform- $d$ ) $\delta 173.7,169.5,167.4,146.0,134.3,131.6,127.7,127.5,125.8,125.1$, 118.8, 63.2, 60.4, 52.4, 40.9, 35.8, 32.9, 32.2, 30.1, 29.8, 29.5, 29.4, 27.6, 25.8, 14.3. Peak overlapping was observed. HRMS (ESI) scaled for $[\mathrm{M}+\mathrm{H}]^{+}: \mathrm{C}_{26} \mathrm{H}_{38} \mathrm{NO}_{5}, \mathrm{~m} / \mathrm{z}$ : 444.2744, observed: 444.2730 .

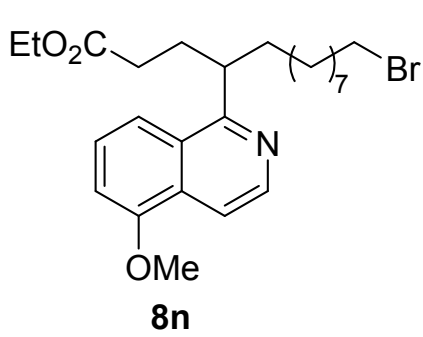

Ethyl 13-bromo-4-(5-methoxyisoquinolin-1-yl)tridecanoate. Colorless oil ( $45.8 \mathrm{mg}, 48 \%$ yield), $0.2 \mathrm{mmol}$ scale reaction. ${ }^{1} \mathbf{H}$ NMR $(500 \mathrm{MHz}$, chloroform- $d$ ) $\delta 8.52(\mathrm{~d}, J=$ $5.8 \mathrm{~Hz}, 1 \mathrm{H}), 7.89(\mathrm{~d}, J=5.8,1 \mathrm{H}), 7.75(\mathrm{~d}, J=8.6 \mathrm{~Hz}, 1 \mathrm{H})$, $7.47(\mathrm{dd}, J=8.6,7.6 \mathrm{~Hz}, 1 \mathrm{H}), 6.98(\mathrm{~d}, J=7.6 \mathrm{~Hz}, 1 \mathrm{H}), 4.08$ -3.97 (comp, 5H), $3.75-3.69(\mathrm{~m}, 1 \mathrm{H}), 3.37(\mathrm{t}, J=6.9 \mathrm{~Hz}$, $2 \mathrm{H}), 2.34-2.22(\mathrm{~m}, 1 \mathrm{H}), 2.19-2.04$ (comp, 3H), $1.99-$ 1.88 (m, 1H), 1.85 - 1.71 (comp, 4H), 1.38 - 1.32 (comp, 2H), 1.25 - 1.13 (comp, $12 \mathrm{H}) .{ }^{13} \mathrm{C}$ NMR $(126 \mathrm{MHz}$, chloroform- $d$ ) $\delta 173.9,163.5,155.2,141.9,129.1,128.8$, 126.9, 116.6, 113.2, 107.1, 60.3, 55.8, 40.8, 35.8, 34.2, 32.9, 32.4, 30.3, 29.8, 29.4, 28.8, 28.3, 27.7, 14.3. Peak overlapping was observed. HRMS (ESI) scaled for $[\mathrm{M}+\mathrm{H}]^{+}$: $\mathrm{C}_{25} \mathrm{H}_{37} \mathrm{BrNO}_{3}, \mathrm{~m} / \mathrm{z}$ : 478.1951, observed: 478.1950 .<smiles>CCOC(=O)CCC(CCCCBr)c1nccc2c(Cl)cccc12</smiles>

Ethyl 13-bromo-4-(5-chloroisoquinolin-1-yl)tridecanoate. Colorless oil (52.0 mg, $54 \%$ yield), $0.2 \mathrm{mmol}$ scale reaction. ${ }^{1} \mathbf{H}$ NMR $(500 \mathrm{MHz}$, chloroform- $d) \delta 8.63(\mathrm{~d}, J=$ $5.9 \mathrm{~Hz}, 1 \mathrm{H}), 8.15(\mathrm{~d}, J=8.6 \mathrm{~Hz}, 1 \mathrm{H}), 7.92(\mathrm{dd}, J=5.9,0.9$ $\mathrm{Hz}, 1 \mathrm{H}), 7.75(\mathrm{dd}, J=7.5,0.9 \mathrm{~Hz}, 1 \mathrm{H}), 7.50(\mathrm{dd}, J=8.6$, $7.5 \mathrm{~Hz}, 1 \mathrm{H}), 4.04(\mathrm{q}, J=7.2 \mathrm{~Hz}, 2 \mathrm{H}), 3.79-3.75(\mathrm{~m}, 1 \mathrm{H})$, $3.37(\mathrm{t}, J=6.9 \mathrm{~Hz}, 2 \mathrm{H}), 2.36-2.24(\mathrm{~m}, 1 \mathrm{H}), 2.21-2.04(\mathrm{comp}, 3 \mathrm{H}), 1.99-1.90(\mathrm{~m}$, 1H), $1.85-1.75$ (comp, 3H), $1.41-1.32$ (comp, $2 \mathrm{H}$ ), $1.25-1.15$ (comp, $13 \mathrm{H}) .{ }^{13} \mathrm{C}$ NMR $(126 \mathrm{MHz}$, chloroform-d) $\delta$ 173.7, 164.6, 143.4, 134.3, 132.0, 129.9, 128.9, $126.8,123.8,115.3,60.3,40.8,35.8,34.2,32.9,32.2,30.3,29.8,29.4,29.4,28.8,28.2$, 27.7, 14.3. HRMS (ESI) scaled for $[\mathrm{M}+\mathrm{H}]^{+}: \mathrm{C}_{24} \mathrm{H}_{34} \mathrm{BrClNO}_{2}, \mathrm{~m} / \mathrm{z}: 482.1456$, observed: 482.1456 .

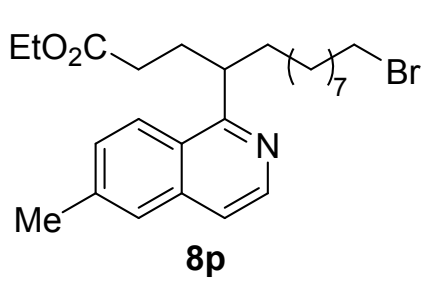

Ethyl 13-bromo-4-(6-methylisoquinolin-1-yl)tridecanoate. Colorless oil (56.2 $\mathrm{mg}, 61 \%$ yield), $0.2 \mathrm{mmol}$ scale reaction. ${ }^{1}$ H NMR $(500 \mathrm{MHz}$, chloroform- $d) \delta 8.46(\mathrm{~d}, J=$ $5.7 \mathrm{~Hz}, 1 \mathrm{H}), 8.08(\mathrm{~d}, J=8.7 \mathrm{~Hz}, 1 \mathrm{H}), 7.57(\mathrm{~s}, 1 \mathrm{H}), 7.46-$ $7.37(\mathrm{~m}, 2 \mathrm{H}), 4.03(\mathrm{q}, J=7.1 \mathrm{~Hz}, 2 \mathrm{H}), 3.75-3.69(\mathrm{~m}, 1 \mathrm{H})$, $3.36(\mathrm{t}, J=6.9 \mathrm{~Hz}, 2 \mathrm{H}), 2.52(\mathrm{~s}, 3 \mathrm{H}), 2.32-2.23(\mathrm{~m}, 1 \mathrm{H})$, $2.19-2.05$ (comp, 3H), $1.98-1.89$ (m, 1H), $1.84-1.68$ (comp, 3H), $1.38-1.31$ 
(comp, 2H), 1.26 - 1.11 (comp, 13H). ${ }^{13} \mathbf{C ~ N M R ~ ( 1 2 6 ~ M H z , ~ c h l o r o f o r m - ~} d$ ) $\delta 173.8$, $163.8,142.3,140.0,136.7,129.3,126.54,126.45,124.6,118.6,60.2$, 40.5, 35.8, 34.1, 32.9, 32.3, 30.3, 29.8, 29.4, 28.8, 28.2, 27.7, 21.9, 14.3. Peak overlapping was observed. HRMS (ESI) scaled for $[\mathrm{M}+\mathrm{H}]^{+}: \mathrm{C}_{25} \mathrm{H}_{37} \mathrm{BrNO}_{2}$, m/z: 462.2002, observed: 462.2001 .

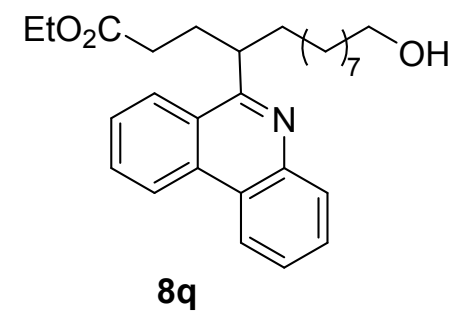

Ethyl 13-hydroxy-4-(phenanthridin-6-yl)tridecanoate.

White solid (79.5 mg, 91\% yield), $0.2 \mathrm{mmol}$ scale reaction. m.p. 82.6-83.6 ${ }^{\circ} \mathrm{C} .{ }^{1} \mathbf{H}$ NMR $(500 \mathrm{MHz}$, chloroform- $d$ ) $\delta$ $8.67(\mathrm{dd}, J=8.3,1.2 \mathrm{~Hz}, 1 \mathrm{H}), 8.55(\mathrm{dd}, J=8.2,1.3 \mathrm{~Hz}$, $1 \mathrm{H}), 8.31(\mathrm{~d}, J=8.3 \mathrm{~Hz}, 1 \mathrm{H}), 8.13(\mathrm{dd}, J=8.2,1.3 \mathrm{~Hz}$, $1 \mathrm{H}), 7.83$ (ddd, $J=8.2,6.9,1.2 \mathrm{~Hz}, 1 \mathrm{H}), 7.72-7.67$ (comp, $2 \mathrm{H}), 7.62(\mathrm{ddd}, J=8.3,6.9,1.3 \mathrm{~Hz}, 1 \mathrm{H}), 4.03(\mathrm{q}, J=7.1 \mathrm{~Hz}, 2 \mathrm{H}), 3.87-3.80(\mathrm{~m}, 1 \mathrm{H})$, $3.59(\mathrm{t}, J=6.7 \mathrm{~Hz}, 2 \mathrm{H}), 2.57-2.42(\mathrm{~m}, 1 \mathrm{H}), 2.33-2.27$ (m, 1H), $2.23-2.14$ (comp, $2 \mathrm{H}), 2.12-1.98(\mathrm{~m}, 1 \mathrm{H}), 1.85-1.72(\mathrm{~m}, 1 \mathrm{H}), 1.53-1.48$ (comp, 2H), $1.30-1.19$ (comp, 12H), 1.15 (t, $J=7.1 \mathrm{~Hz}, 3 \mathrm{H}) .{ }^{13} \mathbf{C}$ NMR (126 MHz, chloroform- $d$ ) $\delta$ 174.1, 163.8, 143.9, 133.1, 130.2, 130.1, 128.5, 127.3, 126.4, 126.1, 125.6, 123.4, 122.7, 121.9, $63.1,60.3,40.8,35.4,32.9,32.4,29.9,29.6,29.4,27.7,25.8$, 14.3. Peak overlapping was observed. HRMS (ESI) scaled for $[\mathrm{M}+\mathrm{H}]^{+}: \mathrm{C}_{28} \mathrm{H}_{38} \mathrm{NO}_{3}, \mathrm{~m} / \mathrm{z}: 436.2846$, observed: 436.2846.

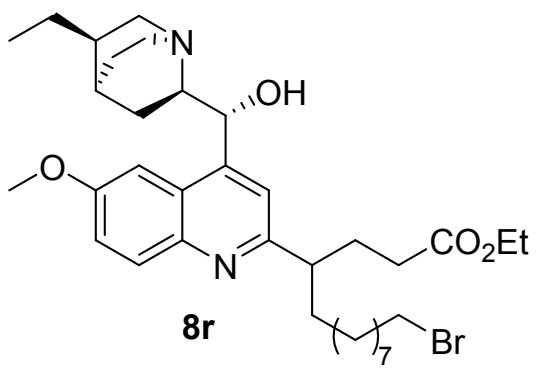

Ethyl 13-bromo-4-\{4-[(R)-[(1S,2R,4S,5R)-5-ethylquinuclidin-2-yl](hydroxy)methyl]-6methoxyquinolin-2-yl\}tridecanoate. Colorless oil (79.9 mg, $d r: 1: 1,62 \%$ yield), $0.2 \mathrm{mmol}$ scale reaction. The $d r$ value was determined by ${ }^{1} \mathrm{H}$ NMR. ${ }^{1}$ H NMR (500 MHz, chloroform- $d$ ) $\delta 7.96-7.94$ (comp, 2H), $7.50(\mathrm{~s}, 1 \mathrm{H}), 7.45(\mathrm{~s}, 1 \mathrm{H}), 7.31-7.29$ (comp, 2H), $7.24-7.21$ (comp, 2H), 5.63 (t, $J=3.7 \mathrm{~Hz}, 2 \mathrm{H}), 3.98$ (q, $J=7.2 \mathrm{~Hz}, 2 \mathrm{H}$ ), $3.97-3.89$ (comp, 2H), $3.89-3.87$ (comp, 6H), $3.62-3.45$ (comp, 2H), $3.37-3.34$ (comp, 4H), $3.10-3.03$ (comp, 4H), $2.89-2.81$ (comp, 2H), 2.66 - 2.59 (comp, 2H), $2.41-2.36$ (comp, 2H), $2.21-2.15$ (comp, 2H), 2.12 - 1.99 (comp, 3H), $1.99-1.91$ (comp, 2H), 1.82 - 1.66 (comp, 15H), 1.42 - 1.30 (comp, 10H), 1.27 - 1.10 (comp, $30 \mathrm{H}), 0.78(\mathrm{t}, J=7.2 \mathrm{~Hz}, 6 \mathrm{H}) .{ }^{13} \mathbf{C}$ NMR (126 MHz, chloroform- $d$ ) $\delta$ 174.0, 173.9, 162.0, 161.9, 157.5, 157.4, 148.2, 147.9, 144.2, 144.0, 131.5, 131.4, 125.3, 125.2, 121.1, 118.0, 117.1, 101.5, 72.1, 60.4, 60.3, 59.8, 58.9, 58.8, 55.9, 48.3, 47.9, 43.7, 43.6, 37.6, $35.8,35.5,34.1,32.9,32.6,30.8,30.4,29.83,29.79,29.7,29.54,29.51,29.45,28.8$, 28.3, 28.2 27.8, 27.7, 27.6, 25.8, 25.7, 20.7, 20.5, 14.3, 14.2, 12.2. Peak overlapping was observed. HRMS (ESI) scaled for $[\mathrm{M}+\mathrm{H}]^{+}: \mathrm{C}_{35} \mathrm{H}_{54} \mathrm{BrN}_{2} \mathrm{O}_{4}, \mathrm{~m} / \mathrm{z}: 645.3261$, observed: 645.3237 .

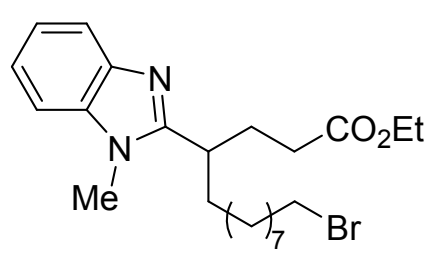

$8 s$
Ethyl 13-bromo-4-(1-methyl-1H-benzo[d]imidazol-2yl)tridecanoate. Colorless oil (23.5 $\mathrm{mg}, 26 \%$ yield), 0.2 mmol scale reaction. ${ }^{1} \mathbf{H}$ NMR (500 MHz, chloroform- $d$ ) $\delta$ $7.78-7.70(\mathrm{~m}, 1 \mathrm{H}), 7.35-7.29(\mathrm{~m}, 1 \mathrm{H}), 7.28-7.26(\mathrm{~m}$, $1 \mathrm{H}), 7.27-7.21(\mathrm{~m}, 1 \mathrm{H}), 4.10-4.00(\mathrm{~m}, 2 \mathrm{H}), 3.75(\mathrm{~s}, 3 \mathrm{H})$, $3.38(\mathrm{t}, J=6.9 \mathrm{~Hz}, 2 \mathrm{H}), 3.10(\mathrm{tt}, J=9.0,5.2 \mathrm{~Hz}, 1 \mathrm{H}), 2.38$ $-2.30(\mathrm{~m}, 1 \mathrm{H}), 2.28-2.22(\mathrm{~m}, 1 \mathrm{H}), 2.21-2.16(\mathrm{~m}, 1 \mathrm{H})$, 
$2.14-2.07$ (m, 1H), $1.94-1.88(\mathrm{~m}, 1 \mathrm{H}), 1.84-1.78$ (comp, 3H), $1.76-1.71(\mathrm{~m}, 1 \mathrm{H})$, $1.40-1.33$ (comp, 3H), 1.27 - 1.19 (comp, 11H). ${ }^{13}$ C NMR (126 MHz, chloroform- $d$ ) $\delta 173.6,157.8,142.8,135.6,122.1,122.0,119.4,109.2,60.5,36.5,35.0,34.2,32.9$, $31.9,29.8,29.7,29.6,29.5,28.8,28.3,27.6,14.4$. Peak overlapping was observed. HRMS (ESI) scaled for [M+H] $: \mathrm{C}_{23} \mathrm{H}_{36} \mathrm{BrN}_{2} \mathrm{O}_{2}, \mathrm{~m} / \mathrm{z}$ : 451.1955, observed: 451.1941.<smiles>CCOC(=O)CCC(CCCCBr)c1cc([N+](=O)[O-])c(C(=O)OCC)nc1C</smiles>

Ethyl 6-(13-bromo-1-ethoxy-1-oxotridecan-4-yl)2,4-dimethylnicotinate. Colorless oil $(27.3 \mathrm{mg}, 33 \%$ yield), $0.2 \mathrm{mmol}$ scale reaction. ${ }^{1} \mathbf{H}$ NMR $(500 \mathrm{MHz}$, chloroform- $d$ ) $\delta 6.76(\mathrm{~s}, 1 \mathrm{H}), 4.40(\mathrm{q}, J=7.1 \mathrm{~Hz}, 2 \mathrm{H})$, $4.07(\mathrm{qd}, J=7.2,0.9 \mathrm{~Hz}, 2 \mathrm{H}), 3.39(\mathrm{t}, J=6.9 \mathrm{~Hz}, 2 \mathrm{H})$, $2.65(\mathrm{tt}, J=8.9,5.8 \mathrm{~Hz}, 1 \mathrm{H}), 2.51(\mathrm{~s}, 3 \mathrm{H}), 2.30(\mathrm{~s}, 3 \mathrm{H})$, $2.20-2.04$ (comp, 2H), $2.03-1.91$ (comp, 2H), $1.88-$

1.78 (comp, 2H), 1.40 (t, $J=7.1 \mathrm{~Hz}, 3 \mathrm{H}), 1.30-1.15$ (comp, $11 \mathrm{H}) .{ }^{13}$ C NMR $(126$ $\mathrm{MHz}$, chloroform- $d$ ) $\delta 173.8,169.3,164.6,154.8,144.9,127.3,121.3,61.4,60.3,47.3$, $35.5,34.2,33.0,32.5,30.4,29.7,29.5,28.8,28.3,27.5,23.2,19.7,14.4,14.4$. Peak overlapping was observed. HRMS (ESI) scaled for $[\mathrm{M}+\mathrm{H}]^{+}: \mathrm{C}_{25} \mathrm{H}_{41} \mathrm{BrNO}_{4}, \mathrm{~m} / \mathrm{z}$ : 498.2213, observed: 498.2201 .

\section{Mechanistic Studies}

\section{a) Reaction in the presence of radical scavenger TEMPO}

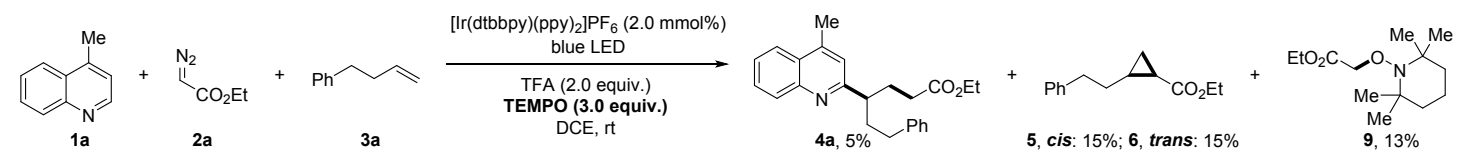

To a reaction vial equipped with a magnetic stir bar were added $\operatorname{Ir}(\mathrm{dtbbpy})(\mathrm{ppy})_{2} \mathrm{PF}_{6}$ (1.8 mg, $0.002 \mathrm{mmol}, 1 \mathrm{~mol} \%$ ), TEMPO (93.8 mg, $0.6 \mathrm{mmol}, 3.0$ equiv.), anhydrous DCE (2.0 mL), lepidine 1a $(0.2 \mathrm{mmol}, 1.0$ equiv.), alkene 3a (0.4 mmol, 2.0 equiv.) and TFA (45.6 mg, $0.4 \mathrm{mmol}, 2.0$ equiv.). The mixture was degassed for 6 minutes by bubbling $\mathrm{N}_{2}$ through the solution. The vial was then placed on a stir plate directly in front of two blue LED lamps (Kessil PR160L-467 nm, 50\% intensity) approximately $8.5 \mathrm{~cm}$ away. The solution of diazo compound 2a ( $0.4 \mathrm{mmol}, 2.0$ equiv.) in DCE (1.0 $\mathrm{mL}$ ) was slowly added into the reaction mixture via a syringe pump over $10 \mathrm{~h}$ under a $\mathrm{N}_{2}$ atmosphere. After another $2 \mathrm{~h}, \mathrm{Ir}(\mathrm{dtbbpy})(\mathrm{ppy})_{2} \mathrm{PF}_{6}(1.8 \mathrm{mg}, 0.002 \mathrm{mmol}, 1 \mathrm{~mol} \%)$ and alkene 3a ( $0.4 \mathrm{mmol}, 2.0$ equiv.) were added, and then the reaction mixture was degassed for 6 minutes by bubbling $\mathrm{N}_{2}$. Another dose of diazo compound $\mathbf{2 a}(0.4 \mathrm{mmol}$, 2.0 equiv.) in DCE $(1.0 \mathrm{~mL})$ was slowly added into the reaction mixture via a syringe pump over $10 \mathrm{~h}$. The resulting solution was irradiated for another $2 \mathrm{~h}$. The reaction temperature was approximately $25^{\circ} \mathrm{C}$ with two fans used to assist cooling. The reaction was quenched by adding saturated aqueous $\mathrm{Na}_{2} \mathrm{CO}_{3}(4.0 \mathrm{~mL}), \mathrm{H}_{2} \mathrm{O}(15.0 \mathrm{~mL})$, extracted with DCM (15.0 $\mathrm{mL} \times 3)$, and the combined organic layers were dried over anhydrous $\mathrm{Na}_{2} \mathrm{SO}_{4}$ and then condensed under reduced pressure. The crude mixture was then purified by column chromatography on silica gel with hexanes/ethyl acetate mixtures as eluent to give the corresponding products. 
<smiles>CCOC(=O)CON1C(C)(C)CCCC1(C)C</smiles>

9

Ethyl

2-[(2,2,6,6-tetramethylpiperidin-1-yl)oxy] acetate. ${ }^{5}$ Colorless oil (18.8 mg, 13\% yield), $0.2 \mathrm{mmol}$ scale reaction. ${ }^{\mathbf{1}} \mathbf{H}$ NMR $(500 \mathrm{MHz}$, chloroform- $d$ ) $\delta 4.43$ (s, 2H), 4.20 (q, $J=7.1$ $\mathrm{Hz}, 2 \mathrm{H}), 1.48-1.40$ (comp, 4H), 1.28 (t, $J=7.1 \mathrm{~Hz}, 3 \mathrm{H}), 1.26-$ 1.22 (comp, 2H), 1.15 (s, 12H). ${ }^{13} \mathbf{C}$ NMR (126 MHz, chloroform- $d$ ) $\delta 170.0,75.7,60.6,60.2,39.9,32.9,20.2,17.2,14.3$. HRMS (ESI) scaled for $[\mathrm{M}+\mathrm{H}]^{+}: \mathrm{C}_{13} \mathrm{H}_{26} \mathrm{NO}_{3}, \mathrm{~m} / \mathrm{z}$ : 244.1907, observed: 244.1907 .

b) Radical clock experiment

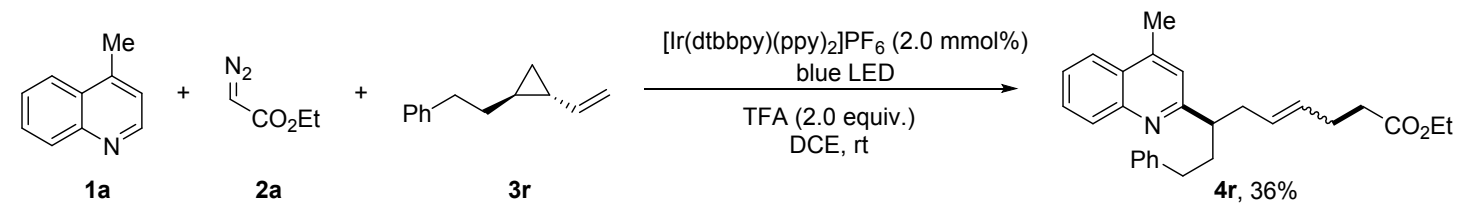

To a reaction vial equipped with a magnetic stir bar were added $\operatorname{Ir}(\mathrm{dtbbpy})(\mathrm{ppy})_{2} \mathrm{PF}_{6}$ (1.8 mg, $0.002 \mathrm{mmol}, 1 \mathrm{~mol} \%$ ), TEMPO (93.8 mg, $0.6 \mathrm{mmol}, 3.0$ equiv.), anhydrous DCE (2.0 mL), lepidine 1a $(0.2 \mathrm{mmol}, 1.0$ equiv.), alkene $3 \mathbf{r}(0.4 \mathrm{mmol}, 2.0$ equiv.) and TFA (45.6 mg, $0.4 \mathrm{mmol}, 2.0$ equiv.). The mixture was degassed for 6 minutes by bubbling $\mathrm{N}_{2}$ through the solution. The vial was then placed on a stir plate directly in front of two blue LED lamps (Kessil PR160L-467 nm, 50\% intensity) approximately $8.5 \mathrm{~cm}$ away. The solution of diazo compound 2a (0.4 mmol, 2.0 equiv.) in DCE (1.0 $\mathrm{mL}$ ) was slowly added into the reaction mixture via a syringe pump over $10 \mathrm{~h}$ under a $\mathrm{N}_{2}$ atmosphere. After another $2 \mathrm{~h}, \mathrm{Ir}(\mathrm{dtbbpy})(\mathrm{ppy})_{2} \mathrm{PF}_{6}(1.8 \mathrm{mg}, 0.002 \mathrm{mmol}, 1 \mathrm{~mol} \%)$ and alkene $3 \mathbf{r}$ ( $0.4 \mathrm{mmol}, 2.0$ equiv.) were added, and then the reaction mixture was degassed for 6 minutes by bubbling $\mathrm{N}_{2}$. Another dose of diazo compound $\mathbf{2 a}(0.4 \mathrm{mmol}$, 2.0 equiv.) in DCE $(1.0 \mathrm{~mL})$ was slowly added into the reaction mixture via a syringe pump over $10 \mathrm{~h}$. The resulting solution was irradiated for another $2 \mathrm{~h}$. The reaction temperature was approximately $25^{\circ} \mathrm{C}$ with two fans used to assist cooling. The reaction was quenched by adding saturated aqueous $\mathrm{Na}_{2} \mathrm{CO}_{3}(4.0 \mathrm{~mL}), \mathrm{H}_{2} \mathrm{O}(15.0 \mathrm{~mL})$, extracted with DCM (15.0 mL x 3), and the combined organic layers were dried over anhydrous $\mathrm{Na}_{2} \mathrm{SO}_{4}$, and then condensed under reduced pressure. The crude mixture was then purified by column chromatography on silica gel with hexanes/ethyl acetate mixtures as eluent to give the corresponding product.

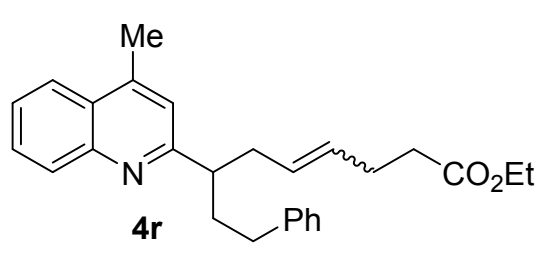

Ethyl 7-(4-methylquinolin-2-yl)-9-phenyl-non-4enoate. Colorless oil ( $29.2 \mathrm{mg}, 36 \%$ yield), $0.2 \mathrm{mmol}$ scale reaction. ${ }^{1} \mathbf{H}$ NMR $(500 \mathrm{MHz}$, chloroform- $d$ ) $\delta$ $8.09(\mathrm{dd}, J=8.4,1.3 \mathrm{~Hz}, 1 \mathrm{H}), 7.97(\mathrm{dd}, J=8.3,1.5$ $\mathrm{Hz}, 1 \mathrm{H}), 7.69$ (ddd, $J=8.4,6.8,1.5 \mathrm{~Hz}, 1 \mathrm{H}), 7.52$ (ddd, $J=8.3,6.8,1.3 \mathrm{~Hz}, 1 \mathrm{H}), 7.25-7.22$ (comp, 2H), $7.18-7.07$ (comp, 4H), $5.40-$ 5.38 (comp, 2H), 4.05 (q, $J=7.1 \mathrm{~Hz}, 2 \mathrm{H}), 3.00$ (dtd, $J=9.4,7.2,5.2 \mathrm{~Hz}, 1 \mathrm{H}), 2.70$ (s, $3 \mathrm{H}$ ), $2.60-2.39$ (comp, 4H), $2.28-2.18$ (comp, 3H), $2.18-2.03$ (comp, 3H), 1.19 (t, $J=7.1 \mathrm{~Hz}, 3 \mathrm{H}) .{ }^{13} \mathbf{C}$ NMR $(126 \mathrm{MHz}$, chloroform- $d$ ) $\delta 173.3,164.7,147.9,144.2$, $142.6,130.2,129.8,129.3,129.0,128.5,128.4,127.2,125.8,125.6,123.7,121.4,60.3$, $48.4,38.8,36.5,34.4,34.0,28.0,19.0,14.3$. The ratio of $E / Z$ isomers is around 4.8:1 
according the integration of the peaks at $142.6 \mathrm{ppm}$ and $142.5 \mathrm{ppm}$ in ${ }^{13} \mathrm{C} \mathrm{NMR} .{ }^{3 \mathrm{f}}$ HRMS (ESI) scaled for $[\mathrm{M}+\mathrm{H}]^{+}: \mathrm{C}_{27} \mathrm{H}_{32} \mathrm{NO}_{2}, \mathrm{~m} / \mathrm{z}$ : 402.2428, observed: 402.2421 .

\section{c) Isotope-labeling experiment}

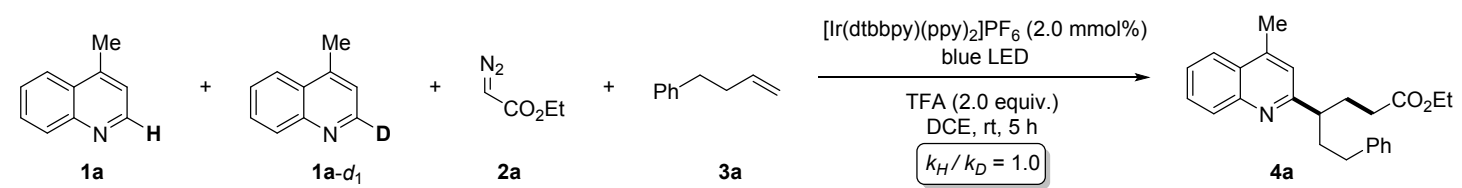

To a reaction vial equipped with a magnetic stir bar were added $\operatorname{Ir}(\mathrm{dtbbpy})(\mathrm{ppy})_{2} \mathrm{PF}_{6}$ (1.8 mg, $0.002 \mathrm{mmol}, 1 \mathrm{~mol} \%)$, anhydrous DCE $(2.0 \mathrm{~mL})$, lepidine $1 \mathbf{a}(0.1 \mathrm{mmol}), 1 \mathrm{a}-$ $d_{1}(0.085 \mathrm{mmol}$ ), alkene $3 \mathbf{a}(0.4 \mathrm{mmol}, 2.0$ equiv.) and TFA (45.6 mg, $0.4 \mathrm{mmol}, 2.0$ equiv.). The mixture was degassed for 6 minutes by bubbling $\mathrm{N}_{2}$ through the solution. The vial was then placed on a stir plate directly in front of two blue LED lamps (Kessil PR160L-467 nm, 50\% intensity) approximately $8.5 \mathrm{~cm}$ away. The solution of diazo compound $2 \mathrm{a}(0.4 \mathrm{mmol}, 2.0$ equiv. $)$ in DCE $(1.0 \mathrm{~mL})$ was slowly added into the reaction mixture via a syringe pump over $10 \mathrm{~h}$ under a $\mathrm{N}_{2}$ atmosphere. The reaction temperature was approximately $25^{\circ} \mathrm{C}$ with two fans used to assist cooling. The reaction was quenched by adding saturated aqueous $\mathrm{Na}_{2} \mathrm{CO}_{3}(4.0 \mathrm{~mL}), \mathrm{H}_{2} \mathrm{O}(15.0 \mathrm{~mL})$, extracted with DCM $(15.0 \mathrm{~mL} \times 3)$, and the combined organic layers were dried over anhydrous $\mathrm{Na}_{2} \mathrm{SO}_{4}$, and then condensed under reduced pressure. The crude mixture was then purified by column chromatography on silica gel with hexanes/ethyl acetate mixtures as eluent to recover the starting material $(12.6 \mathrm{mg}, 44 \%)$ and separate the product (37.0 $\mathrm{mg}, 51 \%$ ). The ratio of $1 \mathbf{a}$ and $1 \mathbf{a}-d_{1}$ was $1: 0.85$, which is identical with that of the starting material. Thus, the consumption ratio of $\mathbf{1 a}$ and $\mathbf{1 a}-d_{1}$ is 1.0 .

\section{Quenching experiments of $\left[\operatorname{Ir}(d t b b p y)(p p y)_{2}\right] P_{6}$}

\section{Characterizations}

UV-visible absorption spectra were recorded on a Shimadzu UV-2600 spectrophotometer. Steady-state emissions spectra were measured on an Edinburgh FLS-1000 photoluminescence spectrometer. Emission lifetime measurements were carried out on a PicoQuant FluoTime 300 time-correlated single-photon counting (TCSPC) spectrophotometer equipped with a Coherent Chameleon Ti:sapphire ultrafast laser as the excitation source. An excitation wavelength of $467 \mathrm{~nm}$ was chosen for all samples in both steady-state and time-resolved measurements. 


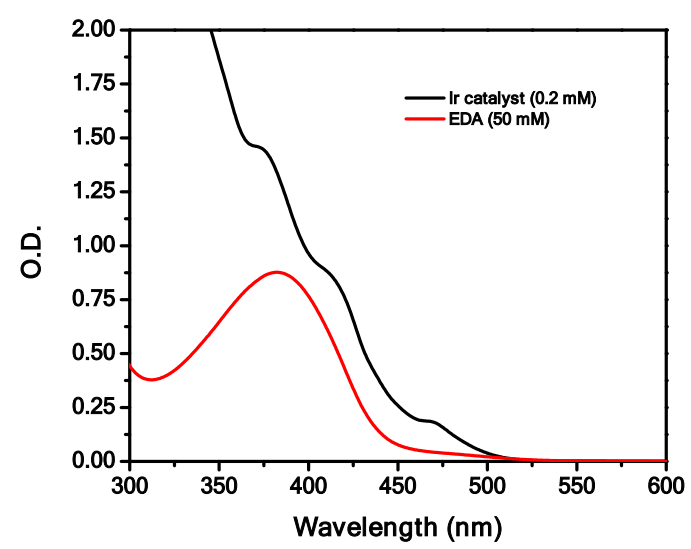

Figure S1. Absorption spectra of $\left[\operatorname{Ir}(\mathrm{dtbbpy})(\mathrm{ppy})_{2}\right] \mathrm{PF}_{6}$ and ethyl diazoacetate (EDA) 2a in 1,2-dichloroethane (DCE). Due to the relatively high concentration in the solution absorption of $\operatorname{Ir}(\mathrm{dtbbpy})(\mathrm{ppy})_{2}{ }^{+}$and EDA are off-scale for wavelengths below $300 \mathrm{~nm}$.

\section{Steady-State Stern-Volmer Quenching Experiments}

Steady-state Stern-Volmer emission quenching experiments were carried out using a $0.01 \mathrm{mM}$ solution of photocatalyst [Ir(dtbbpy)(ppy $\left.)_{2}\right] \mathrm{PF}_{6}$ and variable concentrations $(20,40,60,80,100 \mathrm{mM})$ of ethyl diazoacetate (EDA) 2a, lepidine (LEP) 1a, 4-phenyl1-butene (Alkene) $\mathbf{3 a}$ or TFA in anhydrous 1,2-dichloroethane (DCE). The solution of [Ir(dtbbpy)(ppy $\left.)_{2}\right] \mathrm{PF}_{6}$ in DCE $(0.01 \mathrm{mM}, 4.0 \mathrm{~mL})$ was added to a specially designed long-neck quartz cuvette sealed with a rubber sleeve stopper. The solution was degassed by bubbling with $\mathrm{N}_{2}$ for 6 minutes. EDA, LEP, or Alkene was added via a micro-syringe under $\mathrm{N}_{2}$ protection. The emission spectra of all samples were obtained with a $467 \mathrm{~nm}$ excitation, and the absorption of $\operatorname{Ir}(\mathrm{dtbbpy})(\mathrm{ppy})_{2}{ }^{+}$at this wavelength was 0.011 . In the steady-state Sterm-Volmer plots, the y-axis is expressed as $\mathrm{I}_{0} / \mathrm{I}$, where $\mathrm{I}_{0}$ is the emission intensity of $\left[\operatorname{Ir}(\mathrm{dtbbpy})(\mathrm{ppy})_{2}\right] \mathrm{PF}_{6}$ at $\lambda_{\max }$ in the absence of a quencher, and $\mathrm{I}$ is the observed intensity at $\lambda_{\max }$ with different concentrations of a quencher. 

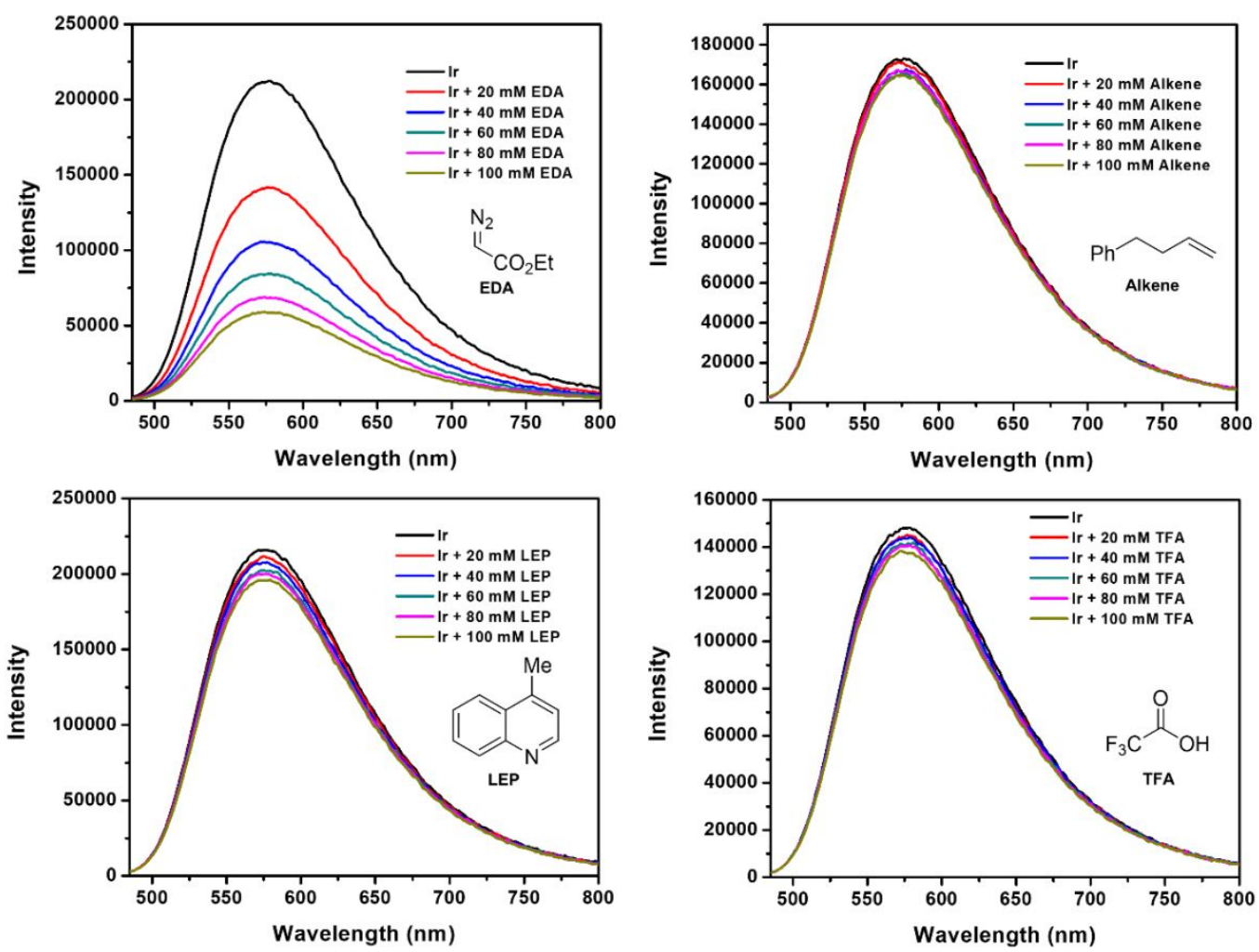

Figure S2. Emission spectra of $\left[\operatorname{Ir}(\mathrm{dtbbpy})(\mathrm{ppy})_{2}\right] \mathrm{PF}_{6}(0.01 \mathrm{mM})$ at different concentrations of $\mathbf{1 a}, \mathbf{2} \mathbf{a}, \mathbf{3} \mathbf{a}$ and TFA in DCE.

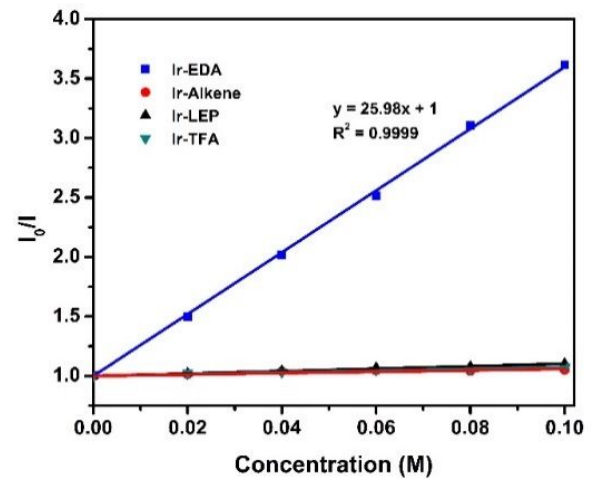

Figure S3. Steady-state emission quenching Stern-Volmer plot for $\left[\operatorname{Ir}(\mathrm{dtbbpy})(\mathrm{ppy})_{2}\right] \mathrm{PF}_{6}(0.01 \mathrm{mM})$ at different concentrations of 1a, 2a, 3a and TFA in DCE. 


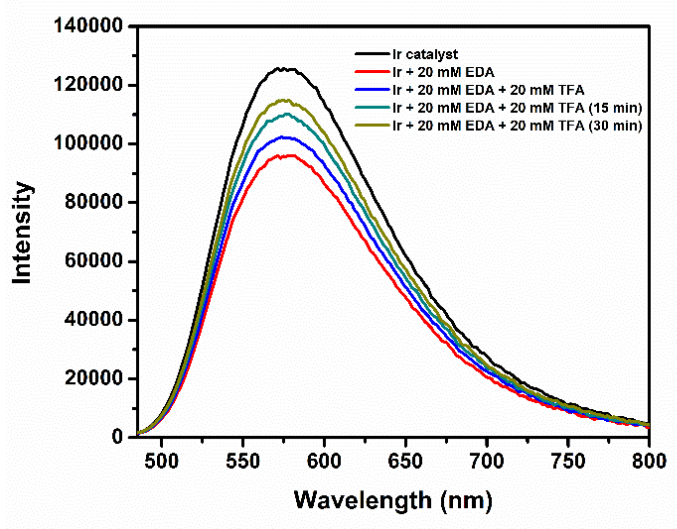

Figure S4. Emission spectra of $\left[\operatorname{Ir}(\mathrm{dtbbpy})(\mathrm{ppy})_{2}\right] \mathrm{PF}_{6}(0.01 \mathrm{mM})$ at the existence of EDA and TFA in DCE.

\section{Time-Resolved Stern-Volmer Quenching Experiments (Photoluminescence Lifetime)}

Time-resolved Stern-Volmer quenching experiments were carried out using a $0.6 \mathrm{mM}$ solution of $\left[\operatorname{Ir}(\mathrm{dtbbpy})(\mathrm{ppy})_{2}\right] \mathrm{PF}_{6}$ and variable concentrations of EDA in DCE (20, 40, $60,80,100 \mathrm{mM})$. The solution of $\left[\operatorname{Ir}(\mathrm{dtbbpy})(\mathrm{ppy})_{2}\right] \mathrm{PF}_{6}$ in DCE $(0.6 \mathrm{mM}, 4 \mathrm{~mL})$ was added to a specially designed long-neck quartz cuvette sealed with a rubber sleeve stopper. The solution was degassed by bubbling with $\mathrm{N}_{2}$ for 6 minutes. EDA was added via a micro-syringe under $\mathrm{N}_{2}$ protection. Photoluminescence decay kinetics were acquired on a PicoQuant FluoTime 300 time-correlated single-photon counting (TCSPC) spectrophotometer equipped with a Coherent Chameleon mode locked Ti: sapphire laser as the excitation source. The Chameleon laser features a pulse picker and frequency doubler. The phosphorescence kinetics were monitored at $575 \mathrm{~nm}\left(\lambda_{\max }{ }^{\mathrm{em}}\right)$ with $467 \mathrm{~nm}$ excitation (100 fs pulse, $0.1 \mathrm{MHz}$ repetition) and fitted to a single exponential decay function to afford the lifetime. In the time-resolved Sterm-Volmer plots, the $\mathrm{y}$-axis is expressed as $\tau^{0} / \tau$, where $\tau^{0}$ is the phosphorescence lifetime of $\left[\operatorname{Ir}(\mathrm{dtbbpy})(\mathrm{ppy})_{2}\right] \mathrm{PF}_{6}$ in the absence of EDA $\left(\tau^{0}=807 \mathrm{~ns}\right)$ and $\tau$ is the observed phosphorescence lifetime of $\left[\operatorname{Ir}(\mathrm{dtbbpy})(\mathrm{ppy})_{2}\right] \mathrm{PF}_{6}$ with different concentrations of EDA.

Table S6. Phosphorescence lifetimes of Ir catalyst at different concentration of EDA.

\begin{tabular}{|c|c|c|c|c|c|c|}
\hline $\begin{array}{c}\text { EDA } \\
\text { concentration }\end{array}$ & $0 \mathrm{mM}$ & $20 \mathrm{mM}$ & $40 \mathrm{mM}$ & $60 \mathrm{mM}$ & $80 \mathrm{mM}$ & $100 \mathrm{mM}$ \\
\hline$\tau / \mathrm{ns}$ & 807 & 558 & 437 & 359 & 300 & 264 \\
\hline $\mathrm{k} / \mathrm{s}^{-1}$ & $1.24 \times 10^{6}$ & $1.79 \times 10^{6}$ & $2.29 \times 10^{6}$ & $2.79 \times 10^{6}$ & $3.33 \times 10^{6}$ & $3.79 \times 10^{6}$ \\
\hline
\end{tabular}

*Lifetimes were fitted with single-exponential decay 


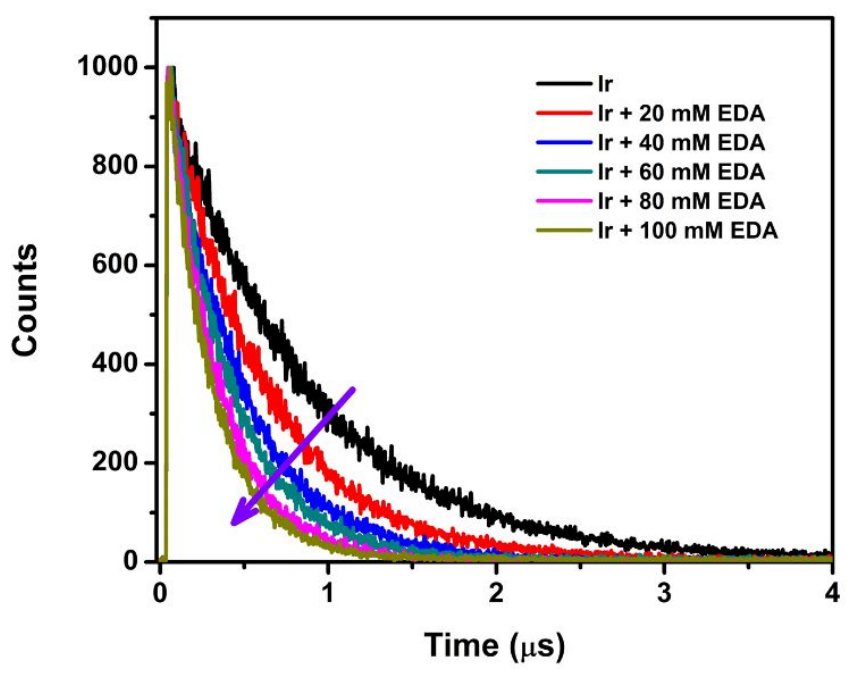

Figure S4. Phosphorescence lifetime of $\left[\operatorname{Ir}(\mathrm{dtbbpy})(\mathrm{ppy})_{2}\right] \mathrm{PF}_{6}(0.6 \mathrm{mM})$ at different concentrations of EDA 2a in DCE. Excitation at $467 \mathrm{~nm}$, detection at $575 \mathrm{~nm}$.

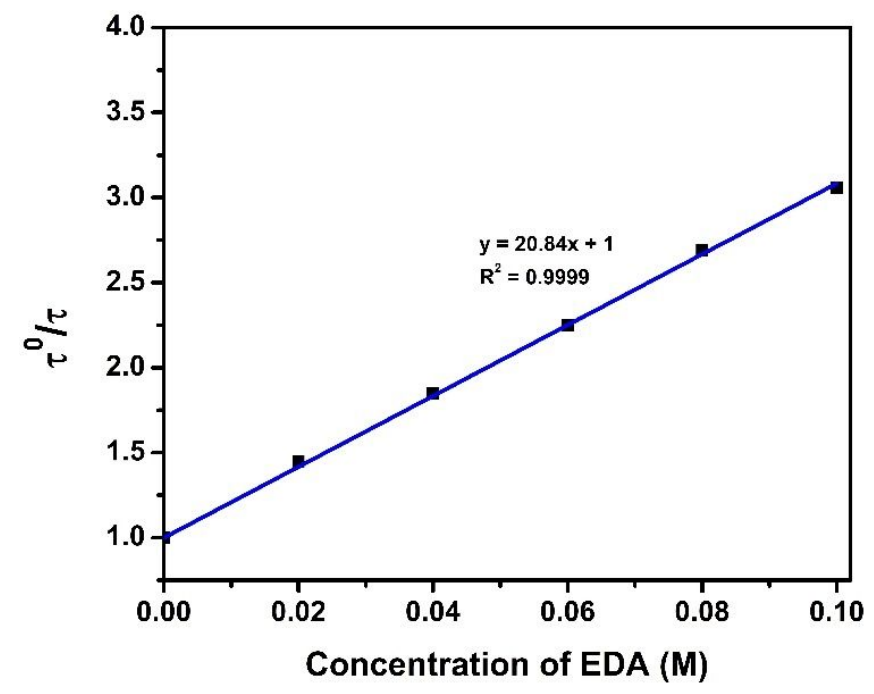

Figure S5. Emission lifetime quenching Stern-Volmer plot for $\left[\operatorname{Ir}(\mathrm{dtbbpy})(\mathrm{ppy})_{2}\right] \mathrm{PF}_{6}$ at different concentrations of EDA $2 \mathbf{a}$ in DCE.

\section{Crystallographic Data and Structure Refinement for Compounds $4 \mathrm{j}$ and $8 \mathrm{q}$}

$4 \mathbf{j}$ : Single crystals of $\mathrm{C}_{25} \mathrm{H}_{36} \mathrm{NO}_{3}$ were prepared by slow evaporation of a mixture of hexane and ethyl acetate. A suitable colorless needle-like crystal, with dimensions of $0.172 \mathrm{~mm} \times 0.079 \mathrm{~mm} \times$ $0.064 \mathrm{~mm}$, was mounted in paratone oil onto a nylon loop. All data were collected at 100.0(1) K, using a XtaLAB Synergy/ Dualflex, HyPix fitted with $\mathrm{CuK} \alpha$ radiation $(\lambda=1.54184 \AA)$. Data collection and unit cell refinement were performed using CrysAlisPro software. ${ }^{6}$ The total number of data were measured in the $7.3^{\circ}<2 \theta<135.0^{\circ}$ using $\omega$ scans. Data processing and absorption correction, giving minimum and maximum transmission factors $(0.770,1.000)$ were accomplished with CrysAlisPro ${ }^{6}$ and $S C A L E 3 A B S P A C K^{7}$, respectively. The structure, using Olex $2^{8}$, was solved with the ShelXT ${ }^{9}$ structure solution program using direct methods and refined (on $F^{2}$ ) with the ShelXL ${ }^{10}$ refinement package using full-matrix, least-squares techniques. All non-hydrogen atoms 
were refined with anisotropic displacement parameters. All hydrogen atom positions were determined by geometry and refined by a riding model.<smiles>CCCCC(CCC(=O)OCC)c1cc(C)c2ccccc2n1</smiles>

$4 \mathbf{j}$

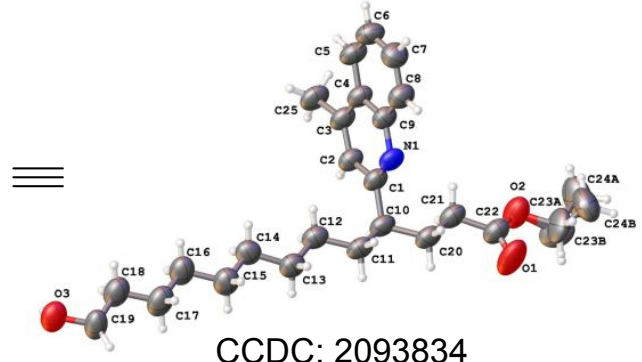

CCDC: 2093834

Table S7. Crystallographic data and structure refinement for $\mathbf{4 j}$

\begin{tabular}{|c|c|}
\hline Identification code & Hp336 \\
\hline Empirical formula & $\mathrm{C}_{25} \mathrm{H}_{36} \mathrm{NO}_{3}$ \\
\hline Formula weight & 398.55 \\
\hline Crystal system & Monoclinic \\
\hline Space group & $C 2 / c$ \\
\hline$a(\hat{A})$ & $24.2586(15)$ \\
\hline$b(\hat{A})$ & $10.3745(7)$ \\
\hline$c(\AA \dot{)})$ & $18.8315(8)$ \\
\hline$\alpha\left(^{\circ}\right)$ & 90 \\
\hline$\beta\left(^{\circ}\right)$ & $93.703(4)$ \\
\hline$\gamma\left({ }^{\circ}\right)$ & 90 \\
\hline Volume $\left(\AA^{3}\right)$ & $4729.4(5)$ \\
\hline $\mathrm{Z}$ & 8 \\
\hline$\rho$ (calc.) & 1.119 \\
\hline$\lambda$ & 1.54184 \\
\hline Temp. (K) & $100.0(1)$ \\
\hline $\mathrm{F}(000)$ & 1736 \\
\hline$\mu\left(\mathrm{mm}^{-1}\right)$ & 0.568 \\
\hline $\mathrm{T}_{\min }, \mathrm{T}_{\max }$ & $0.770,1.000$ \\
\hline
\end{tabular}




\begin{tabular}{|c|c|}
\hline $2 \theta_{\text {range }}\left({ }^{\circ}\right)$ & 7.3 to 135.0 \\
\hline Reflections collected & 25659 \\
\hline Independent reflections & $4250[\mathrm{R}($ int $)=0.0418]$ \\
\hline Completeness & $99.5 \%$ \\
\hline Data / restraints / parameters & $4250 / 0 / 284$ \\
\hline Observed data $[\mathrm{I}>2 \sigma(\mathrm{I})]$ & 3217 \\
\hline$w R\left(F^{2}\right.$ all data $)$ & 0.1672 \\
\hline$R(F$ obsd data) & 0.0947 \\
\hline Goodness-of-fit on $F^{2}$ & 1.07 \\
\hline largest diff. peak and hole $\left(\mathrm{e} \AA^{-3}\right)$ & $0.41 /-0.28$ \\
\hline
\end{tabular}

$$
\begin{gathered}
w R_{2}=\left\{\Sigma\left[w\left(F_{\mathrm{o}}^{2}-F_{\mathrm{c}}^{2}\right)^{2}\right] / \Sigma\left[w\left(F_{\mathrm{o}}{ }^{2}\right)^{2}\right]\right\}^{1 / 2} \\
R_{1}=\Sigma|| F_{\mathrm{o}}|-| F_{\mathrm{c}} \| / \Sigma\left|F_{\mathrm{o}}\right|
\end{gathered}
$$

8q: Single crystals of $\mathrm{C}_{28} \mathrm{H}_{37} \mathrm{NO}_{3}$ were prepared by slow evaporation of a mixture of hexane and ethyl acetate. A suitable colorless needle-like crystal, with dimensions of $0.167 \mathrm{~mm} \times 0.052 \mathrm{~mm} \times$ $0.034 \mathrm{~mm}$, was mounted in paratone oil onto a nylon loop. All data were collected at 100.0(1) K, using a XtaLAB Synergy/ Dualflex, HyPix fitted with $\mathrm{CuK} \alpha$ radiation $(\lambda=1.54184 \AA)$. Data collection and unit cell refinement were performed using CrysAlisPro software. ${ }^{6}$ The total number of data were measured in the $6.5^{\circ}<2 \theta<153.8^{\circ}$ using $\omega$ scans. Data processing and absorption correction, giving minimum and maximum transmission factors $(0.797,1.000)$ were accomplished with $C r y s A l i s P r O^{6}$ and $S C A L E 3 A B S P A C K^{7}$, respectively. The structure, using Olex $2^{8}$, was solved with the ShelXT ${ }^{9}$ structure solution program using direct methods and refined (on $F^{2}$ ) with the ShelXL ${ }^{10}$ refinement package using full-matrix, least-squares techniques. All non-hydrogen atoms were refined with anisotropic displacement parameters. All hydrogen atom positions were determined by geometry and refined by a riding model.

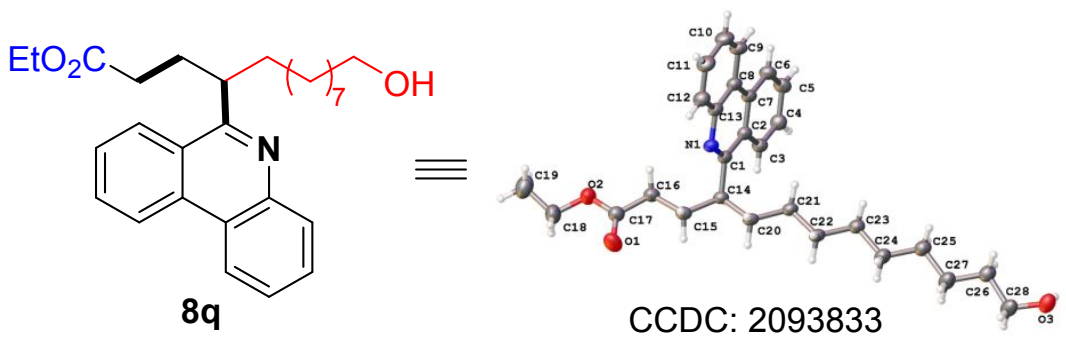

Table S8. Crystallographic data and structure refinement for $\mathbf{8 q}$

\begin{tabular}{|l|c|}
\hline Identification code & Hp335 \\
\hline
\end{tabular}




\begin{tabular}{|c|c|}
\hline Empirical formula & $\mathrm{C}_{28} \mathrm{H}_{37} \mathrm{NO}_{3}$ \\
\hline Formula weight & 435.58 \\
\hline Crystal system & Monoclinic \\
\hline Space group & $C 2 / c$ \\
\hline$a(\AA)$ & $27.2805(5)$ \\
\hline$b(\AA)$ & $9.5063(2)$ \\
\hline$c(\AA)$ & $18.9097(3)$ \\
\hline$\alpha\left(^{\circ}\right)$ & 90 \\
\hline$\beta\left(^{\circ}\right)$ & $92.819(2)$ \\
\hline$\gamma\left({ }^{\circ}\right)$ & 90 \\
\hline Volume $\left(\AA^{3}\right)$ & $4898.04(16)$ \\
\hline Z & 8 \\
\hline$\rho$ (calc.) & 1.181 \\
\hline$\lambda$ & 1.54184 \\
\hline Temp. (K) & $100.0(1)$ \\
\hline $\mathrm{F}(000)$ & 1888 \\
\hline$\mu\left(\mathrm{mm}^{-1}\right)$ & 0.592 \\
\hline $\mathrm{T}_{\min }, \mathrm{T}_{\max }$ & $0.797,1.000$ \\
\hline $2 \theta_{\text {range }}\left({ }^{\circ}\right)$ & 6.5 to 153.8 \\
\hline Reflections collected & 43511 \\
\hline Independent reflections & $5004[\mathrm{R}(\mathrm{int})=0.0663]$ \\
\hline Completeness & $99.8 \%$ \\
\hline Data / restraints / parameters & $5004 / 0 / 291$ \\
\hline Observed data $[\mathrm{I}>2 \sigma(\mathrm{I})]$ & 4337 \\
\hline$w R\left(F^{2}\right.$ all data $)$ & 0.1482 \\
\hline$R(F$ obsd data $)$ & 0.0663 \\
\hline Goodness-of-fit on $F^{2}$ & 1.09 \\
\hline
\end{tabular}




\begin{tabular}{|c|c|}
\hline largest diff. peak and hole $\left(\mathrm{e} \AA^{-3}\right)$ & $0.57 /-0.34$ \\
\hline$w R_{2}=\left\{\Sigma\left[w\left(F_{\mathrm{O}}^{2}-\right.\right.\right.$ & $\left.\left.\left.{ }^{2}\right)^{2}\right]\right\}^{1 / 2}$ \\
\hline
\end{tabular}

\section{References}

1. (a) Proctor, R. S. J.; Davis, H. J.; Phipps, R. J. Catalytic enantioselective Miniscitype addition to heteroarenes. Science 2018, 360, 419-422. (b) Rueping, M.; Ieawsuwan, W. A manganese-catalyzed cross-coupling reaction. Synlett 2007, 2007, 247-250. (c) Leitch, J. A.; Rogova, T.; Duarte, F.; Dixon, D. J. Dearomative photocatalytic construction of bridged 1,3-diazepanes. Angew. Chem., Int. Ed. 2020, 59, 4121-4130. (d) Stephens, D. E.; Nguyen, V. T.; Chhetri, B.; Clark, E. R.; Arman, H. D.; Larionov, O. V. Organocatalytic synthesis of methylene-bridged $N$-heterobiaryls. Org. Lett. 2016, 18, 5808-5811. (e) Preindl, J.; Chakrabarty, S.; Waser, J. Dearomatization of electron poor six-membered $N$-heterocycles through [3+2] annulation with aminocyclopropanes. Chem. Sci. 2017, 8, 7112-7118.

2. (a) Qi, X.; Ready, J. M. Copper-promoted cycloaddition of diazocarbonyl compounds and acetylides. Angew. Chem., Int. Ed. 2007, 46, 3242-3244. (b) Pisella, G.; Gagnebin, A.; Waser, J. Three-component reaction for the synthesis of highly functionalyzed propargyl ethers. Chem. Eur. J. 2020, 26, 10199-10204. (c) Pisella, G.; Gagnebin, A.; Waser, J. Copper-catalyzed oxyvinylation of diazo Compounds. Org. Lett. 2020, 22, 3884-3889. (d) Huang, X.; Webster, R. D.; Harms, Meggers, K. E. Asymmetric catalysis with organic azides and diazo compounds initiated by photoinduced electron transfer. J. Am. Chem. Soc. 2016, 138, 12636-12642. (e) Bode, J. W.; Doyle, M. P.; Protopopova, M. N.; Zhou, Q.-L. Intramolecular regioselective insertion into unactivated prochiral carbon-hydrogen bonds with diazoacetates of primary alcohols catalyzed by ciral dirhodium(II) carboxamidates. highly enantioselective total synthesis of natural lignan lactones. J. Org. Chem. 1996, 61, 9146-9155. (f) Doyle, M. P.; Davies, S. B.; May, E. J. High selectivity from configurational match/mismatch in carbon-hydrogen insertion reactions of steroidal diazoacetates catalyzed by chiral dirhodium(II) carboxamidates. J. Org. Chem. 2001, 66, 8112-8119. (g) Döben, N.; Yan, H.; Kischkewitz, M.; Mao, J.; Studer, A. Intermolecular acetoxyaminoalkylation of $\alpha$-diazo amides with (diacetoxyiodo)benzene and amines Org. Lett. 2018, 20, 7933-7936. (h) Zhang, J.; Chen, W.; Huang, D.; Zeng, X.; Wang, X.; Hu, Y. Tandem synthesis of $\alpha$-diazoketones from 1,3-diketones. J. Org. Chem. 2017, 82, 9171-9174. (i) Baum, J. S.; Shook, D. A.; Davies, H. M. L.; Smith, H. D. Diazotransfer reactions with $p$ acetamidobenzenesulfonyl azide. Synth. Commun. 1987, 17, 1709-1716. (j) Hari, D. P.; Waser, J. Enantioselective copper-catalyzed oxy-alkynylation of diazo compounds. $J$. Am. Chem. Soc. 2017, 139, 8420-8423. (k) Morandi, B.; Carreira, E. M. Synthesis of trifluoroethyl-substituted ketones from aldehydes and cyclohexanones. Angew. Chem., Int. Ed. 2011, 50, 9085-9088.

3. (a) Tappin, N. D. C.; Michalska, W.; Rohrbach, S.; Renaud, P. Cyclopropanation of 
terminal alkenes through sequential atom-transfer radical addition/1,3-elimination. Angew. Chem., Int. Ed. 2019, 58, 14240-14244. (b) Yang, T.; Lu, L.; Shen, Q.-L. Ironmediated markovnikov-selective hydro-trifluoromethylthiolation of unactivated alkenes. Chem. Commun. 2015, 51, 5479-5481. (c) Falk, E.; Makai, S.; Delcaillau, T.; Gürtler, L.; Morandi, B. Design and scalable synthesis of $N$-alkyl-hydroxylamine reagents for the direct, iron-catalyzed installation of medicinally relevant amines. Angew. Chem., Int. Ed. 2020, 59, 21064-21071. (d) Thiedemann, B.; Schmitz, C. M. L.; Staubitz, A. Reduction of $N$-allylamides by $\mathrm{LiAlH}_{4}$ : unexpected attack of the double bond with mechanistic studies of product and byproduct formation. J. Org. Chem. 2014, 79, 10284-10285. (e) Liu, Y.; Wu, H.; Guo, Y.; Xiao, J.-C.; Chen, Q.-Y.; Liu, C. Trifluoromethylfluorosulfonylation of unactivated alkenes using readily available $\mathrm{Ag}\left(\mathrm{O}_{2} \mathrm{CCF}_{2} \mathrm{SO}_{2} \mathrm{~F}\right)$ and $\mathrm{N}$-Fluorobenzenesulfonimide. Angew. Chem., Int. Ed. 2017, 56, 15432-15435. (f) Yang, X.; Tsui, G. C. Trifluoromethylation of unactivated alkenes with $\mathrm{Me}_{3} \mathrm{SiCF}_{3}$ and $\mathrm{N}$-iodosuccinimide. Org. Lett. 2019, 21, 1521-1525.

4. Su, Y.-L.; Liu, G.-X.; Liu, J.-W.; Tram, L.; Qiu, H.; Doyle, M. P. Radical-mediated strategies for the functionalization of alkenes with diazo compounds. J. Am. Chem. Soc. 2020, 142, 13846-13855.

5. Sumino, S.; Fusano, A.; Ryu, I. Reductive bromine atom-transfer reaction. Org. Lett. 2013, 15, 2826-2829.

6. CrysAlisPro 1.171.38.41, Rigaku Oxford Diffraction, 2015.

7. SCALE3 ABSPACK -An Oxford Diffraction program (1.0.4, gui: 1.0.3) (C) 2005 Oxford Diffraction Ltd.

8. Dolomanov, O. V.; Bourhis, L. J.; Gildea, R. J.; Howard, J. A. K.; Puschmann, H. J. Appl. Cryst. 2009, 42, 339-341.

9. Sheldrick, G. M. Acta Cryst. 2015, A71, 3-8.

10. Sheldrick, G. M. Acta Cryst. 2008, A64, 112-122. 


\section{NMR Spectrum of Products}

${ }^{1} \mathrm{H}$ NMR (400 $\left.\mathrm{MHz}, \mathrm{CDCl}_{3}\right)$ and ${ }^{13} \mathrm{C}$ NMR (125 $\left.\mathrm{MHz}, \mathrm{CDCl}_{3}\right)$ spectra for 4a

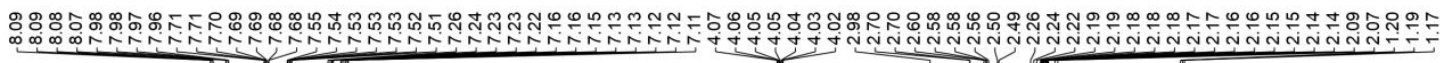
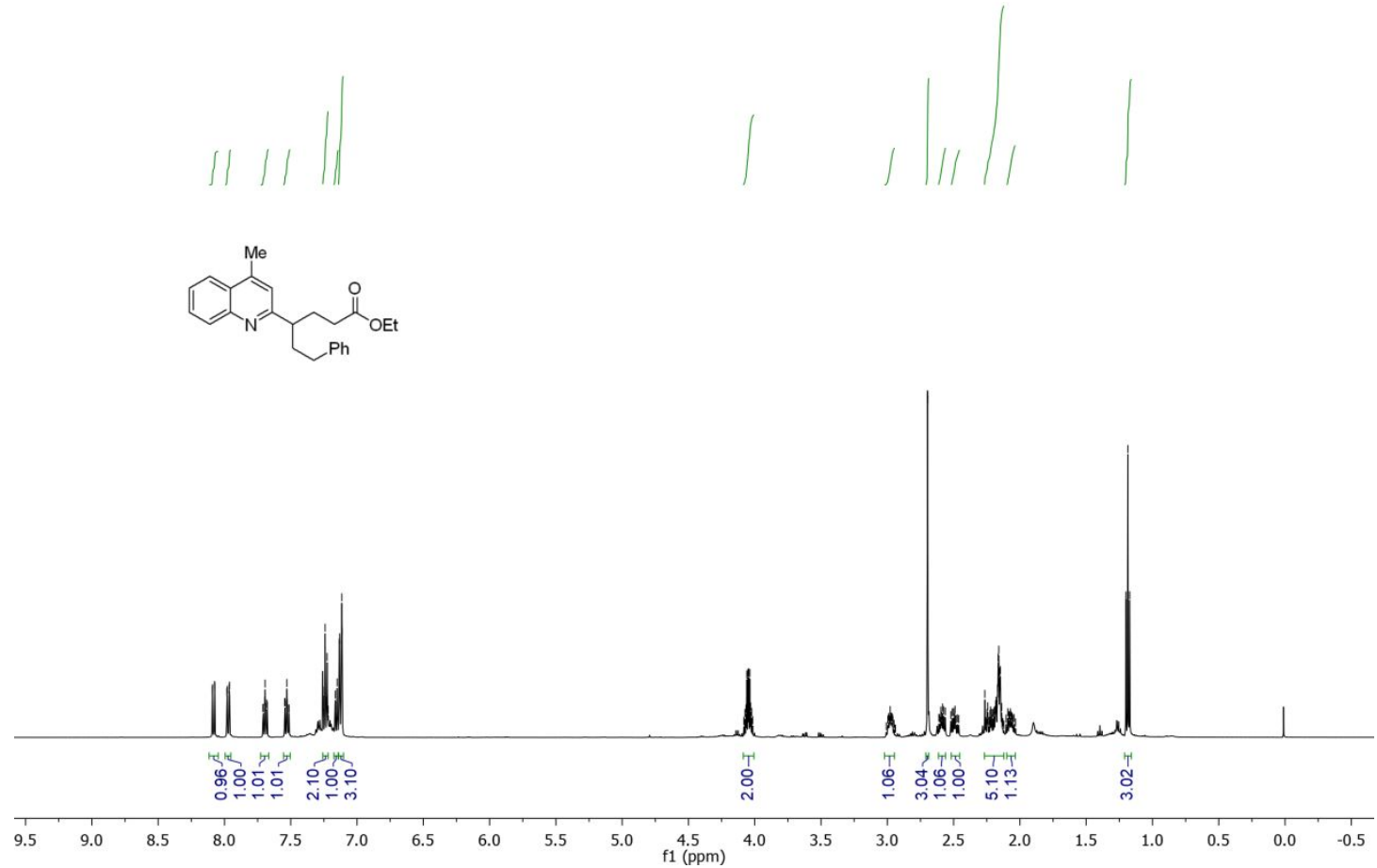

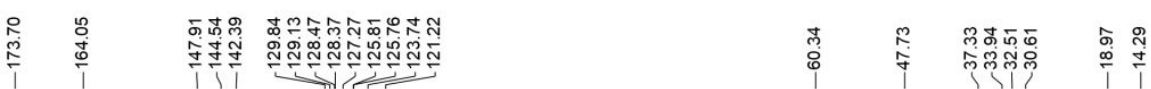
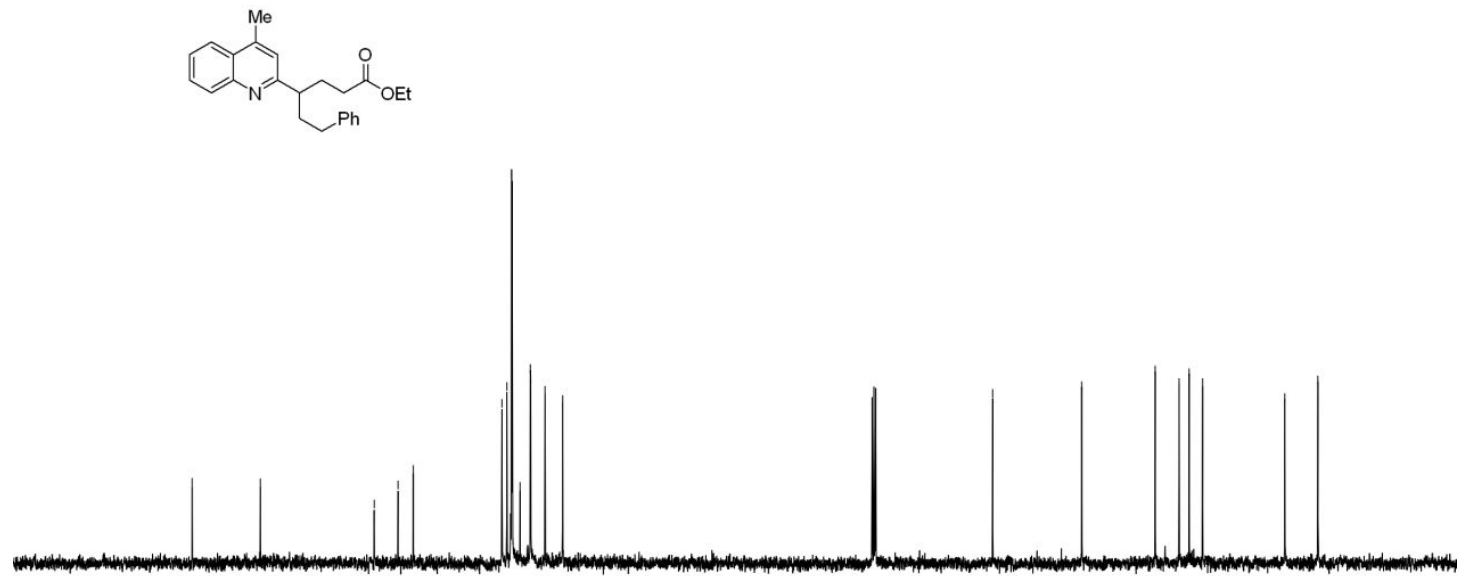
${ }^{1} \mathrm{H}$ NMR (400 MHz, $\left.\mathrm{CDCl}_{3}\right)$ and ${ }^{13} \mathrm{C}$ NMR (125 $\left.\mathrm{MHz}, \mathrm{CDCl}_{3}\right)$ spectra for $4 \mathrm{~b}$

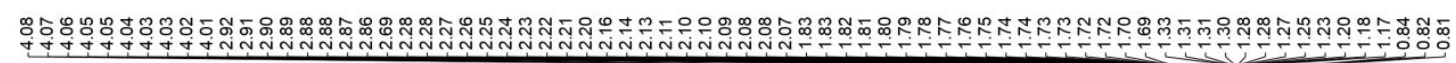
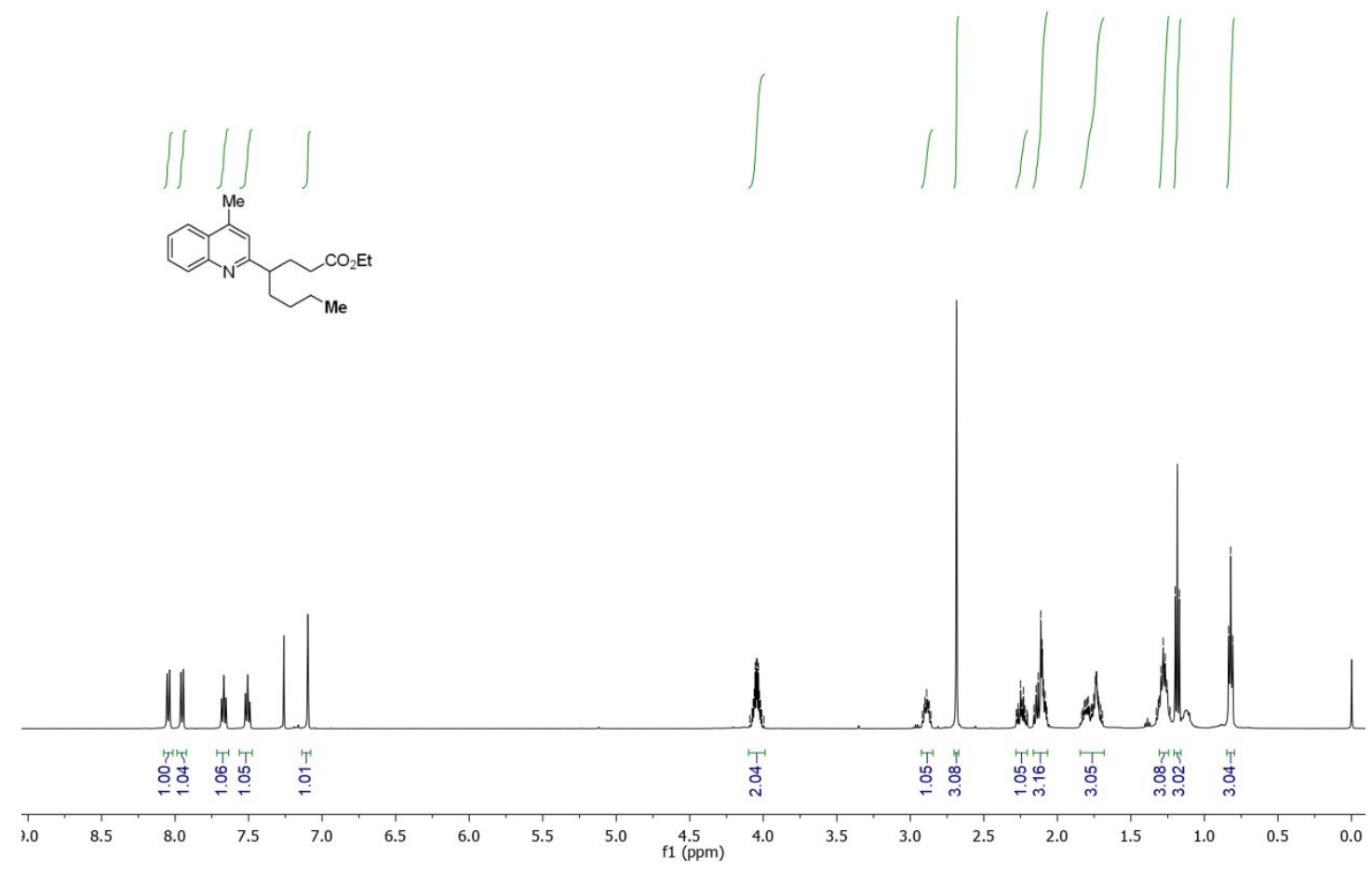

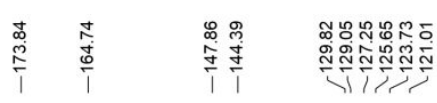

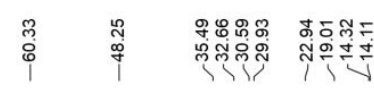
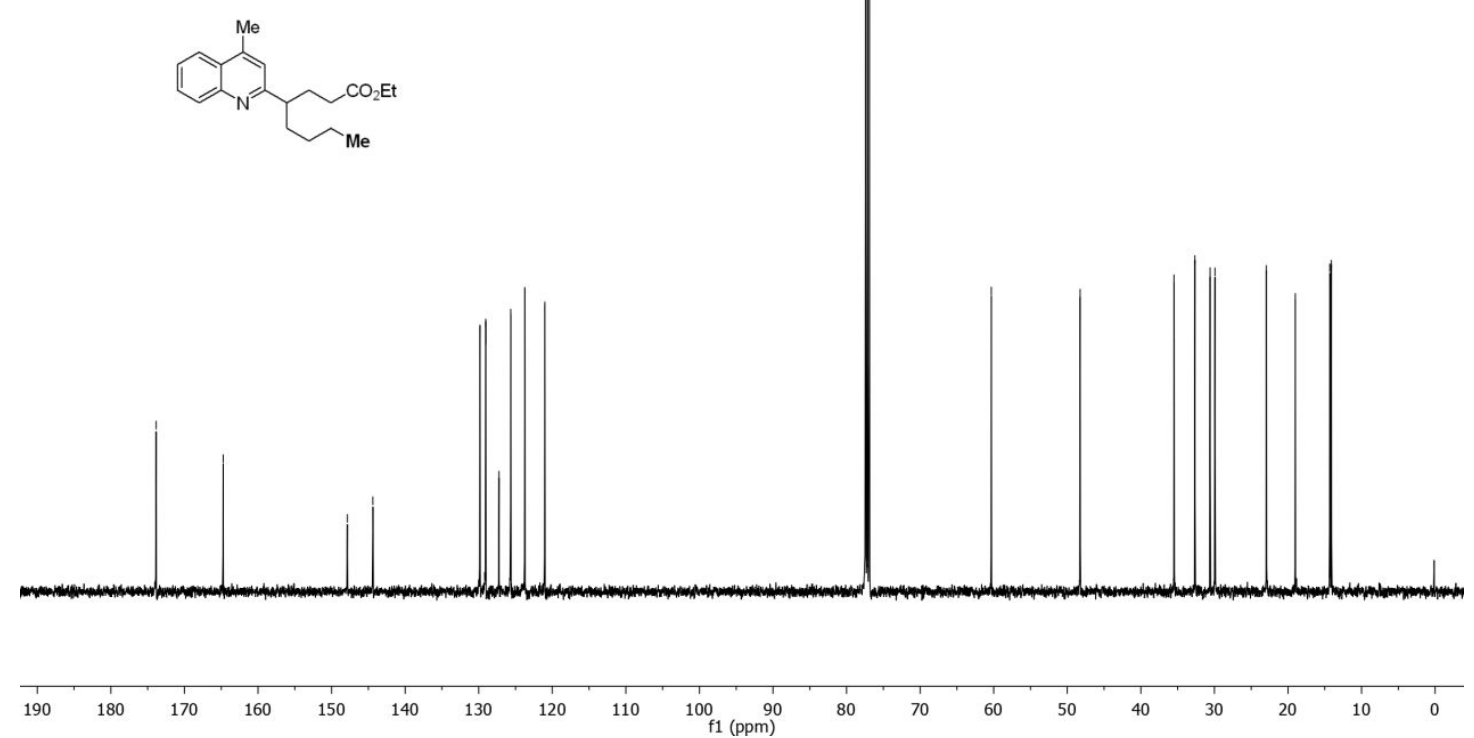
${ }^{1} \mathrm{H}$ NMR (400 MHz, $\left.\mathrm{CDCl}_{3}\right)$ and ${ }^{13} \mathrm{C}$ NMR (125 MHz, $\left.\mathrm{CDCl}_{3}\right)$ spectra for 4c

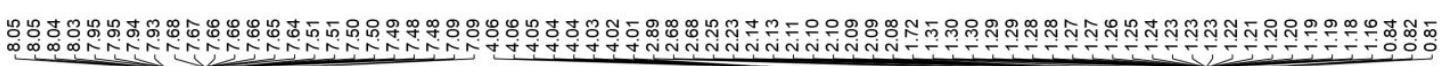

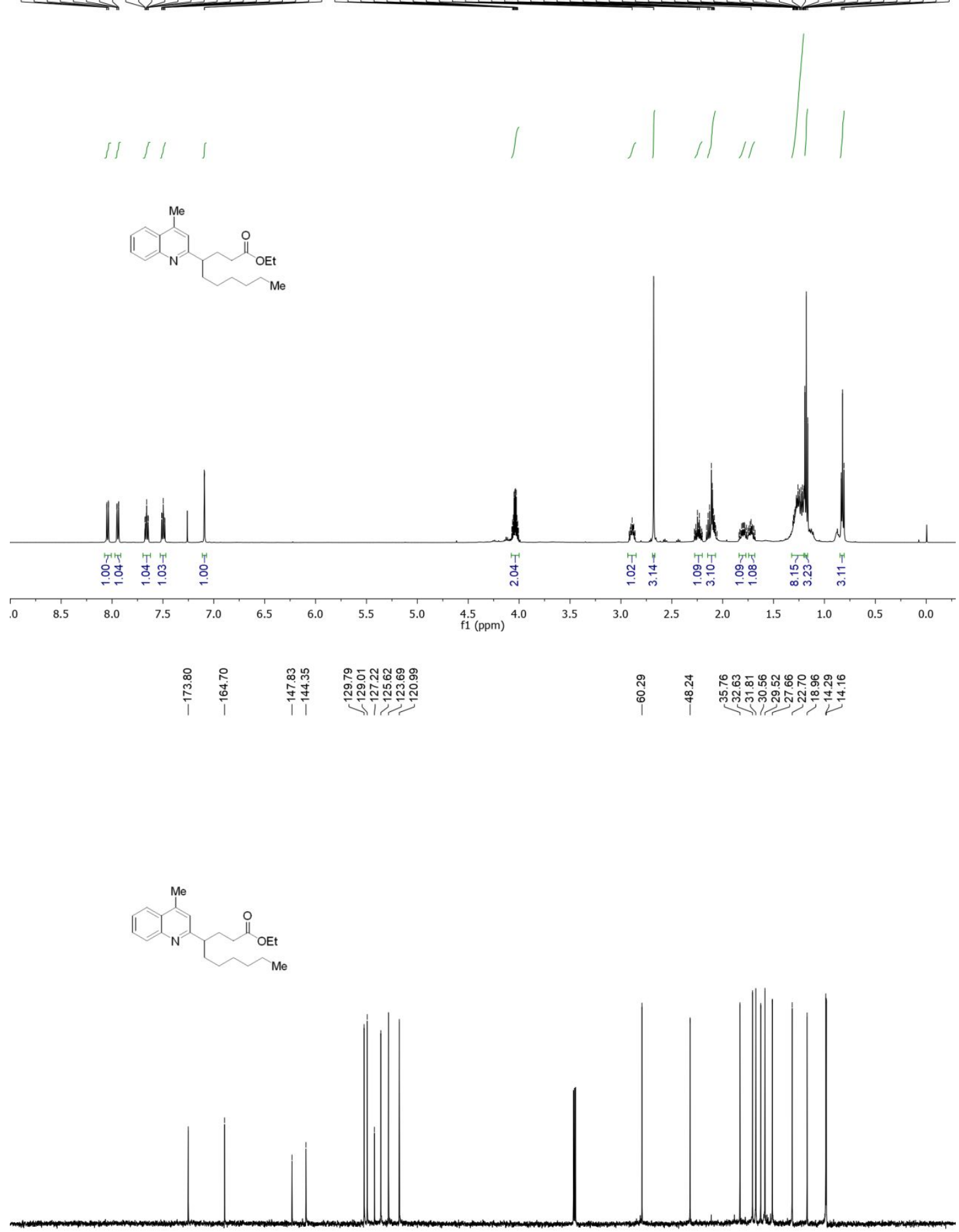

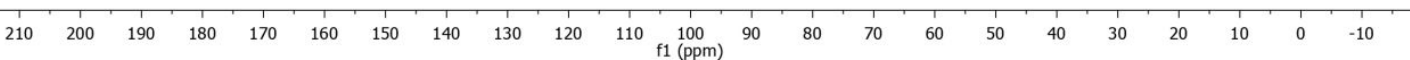


${ }^{1} \mathrm{H}$ NMR (400 MHz, $\left.\mathrm{CDCl}_{3}\right)$ and ${ }^{13} \mathrm{C}$ NMR (125 MHz, $\left.\mathrm{CDCl}_{3}\right)$ spectra for 4d

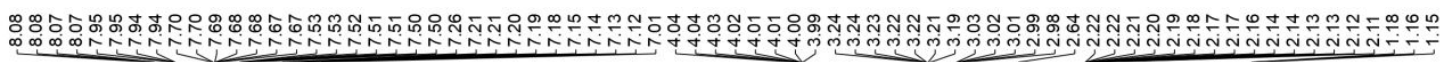
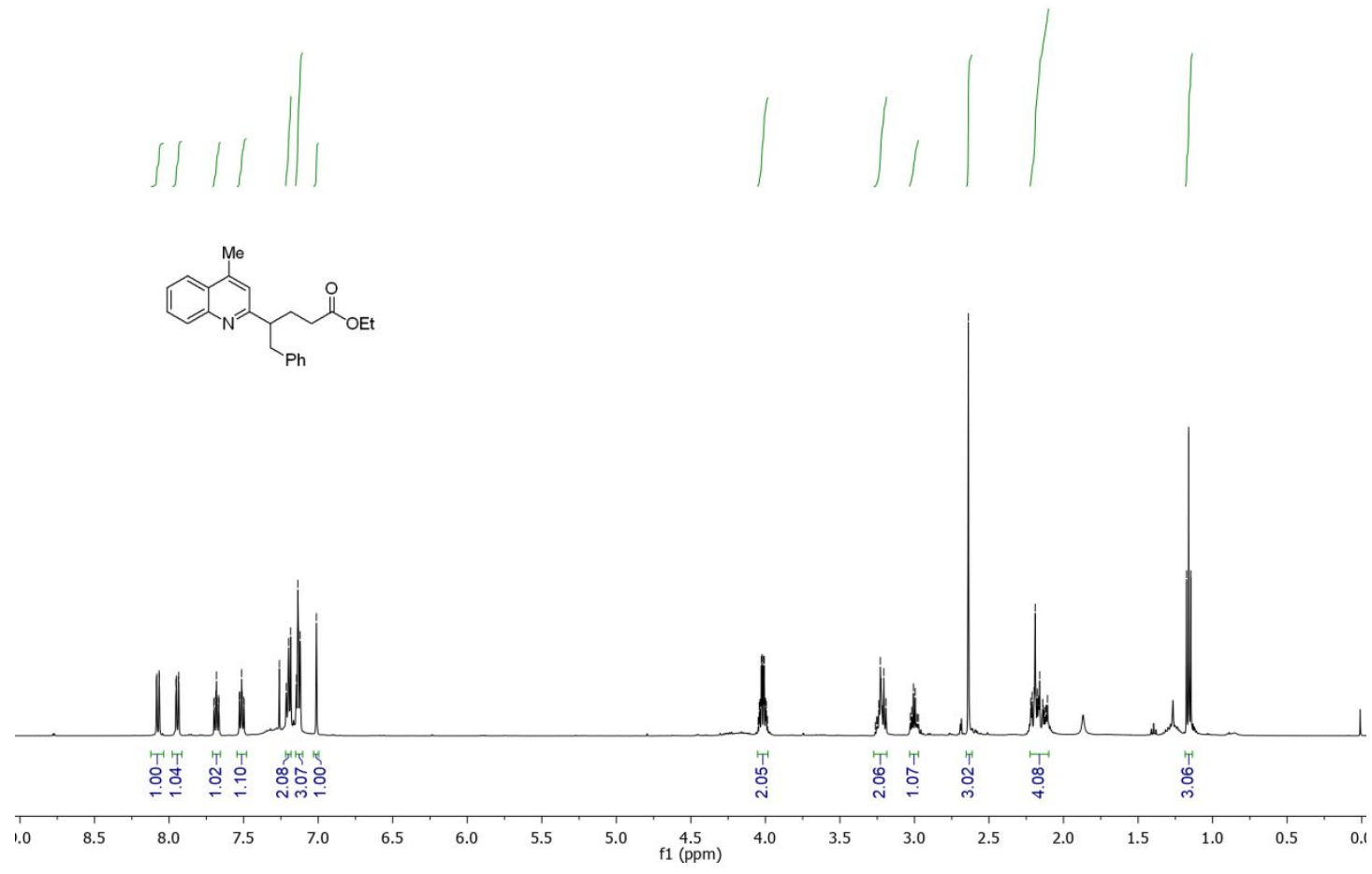

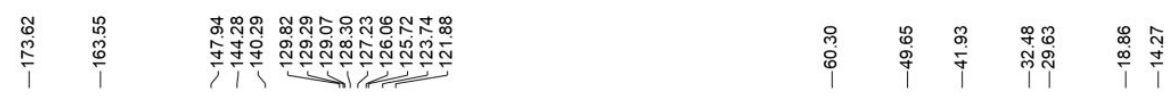

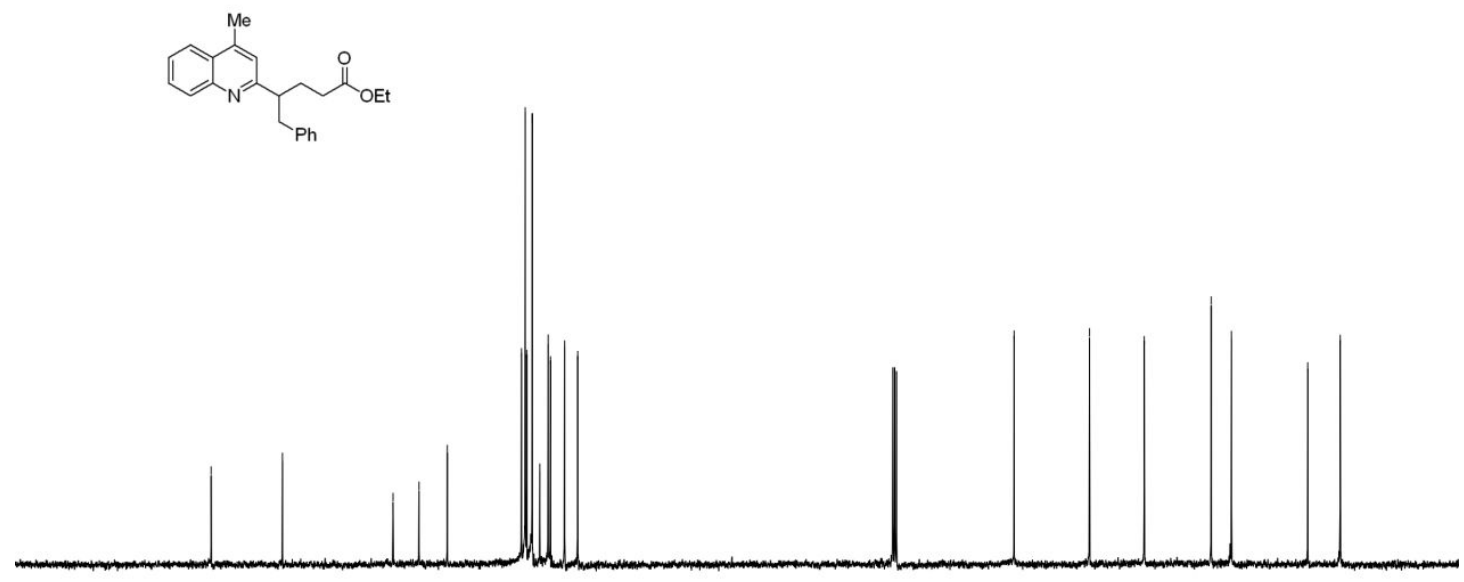

$\begin{array}{lllllllllllllllllllll}1 & 1 \\ 200 & 190 & 180 & 170 & 160 & 150 & 140 & 130 & 120 & 110 & \begin{array}{c}100 \\ \mathrm{f} 1(\mathrm{ppm})\end{array} & 90 & 80 & 70 & 60 & 50 & 40 & 30 & 20 & 10 & 0\end{array}$ 
${ }^{1} \mathrm{H}$ NMR (400 MHz, $\left.\mathrm{CDCl}_{3}\right)$ and ${ }^{13} \mathrm{C}$ NMR (125 MHz, $\left.\mathrm{CDCl}_{3}\right)$ spectra for $4 \mathrm{e}$ $\underbrace{00000}$
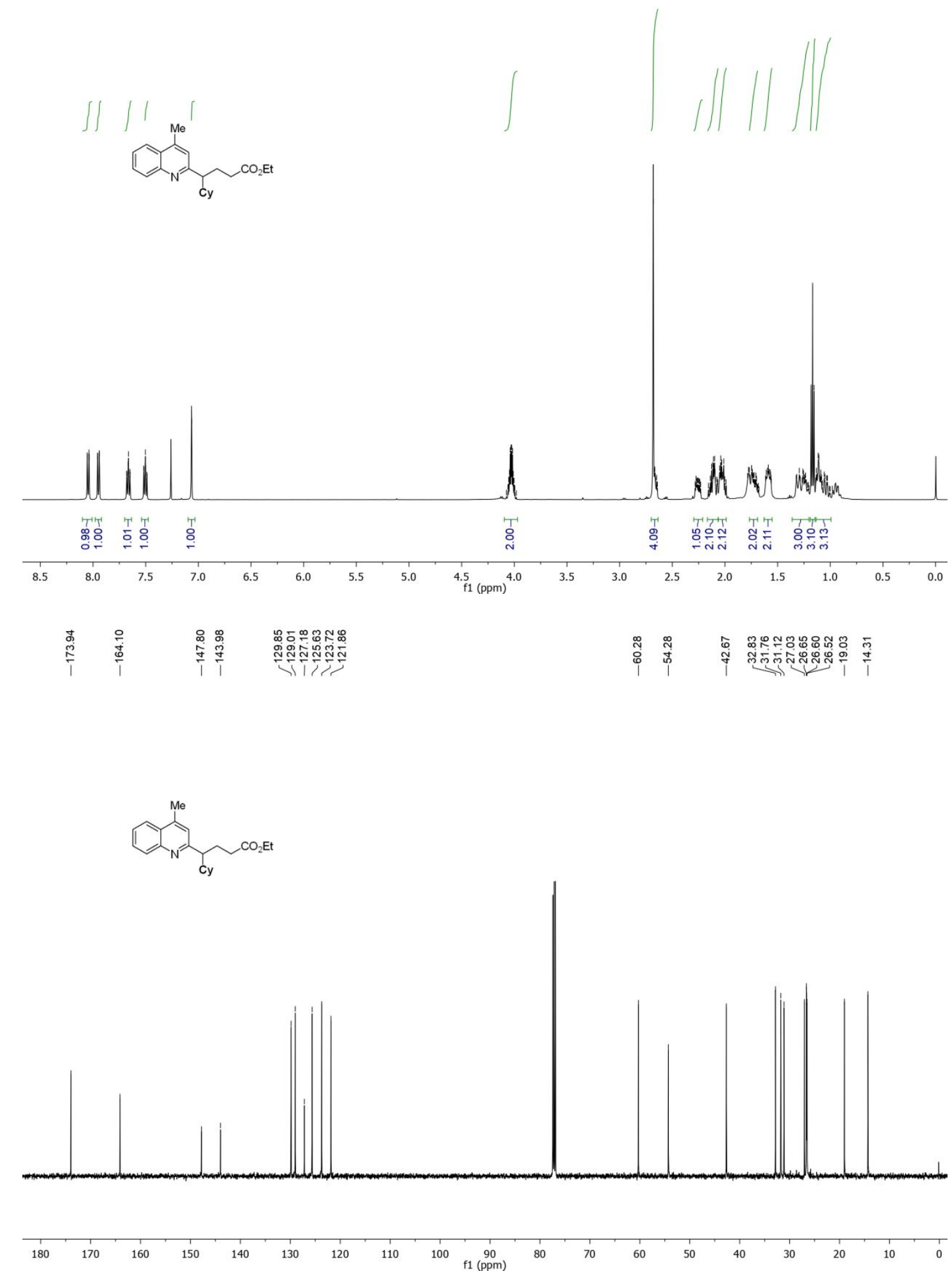
${ }^{1} \mathrm{H}$ NMR (400 MHz, $\left.\mathrm{CDCl}_{3}\right)$ and ${ }^{13} \mathrm{C}$ NMR (125 MHz, $\left.\mathrm{CDCl}_{3}\right)$ spectra for $4 \mathrm{f}$

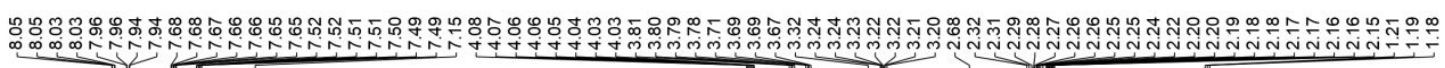

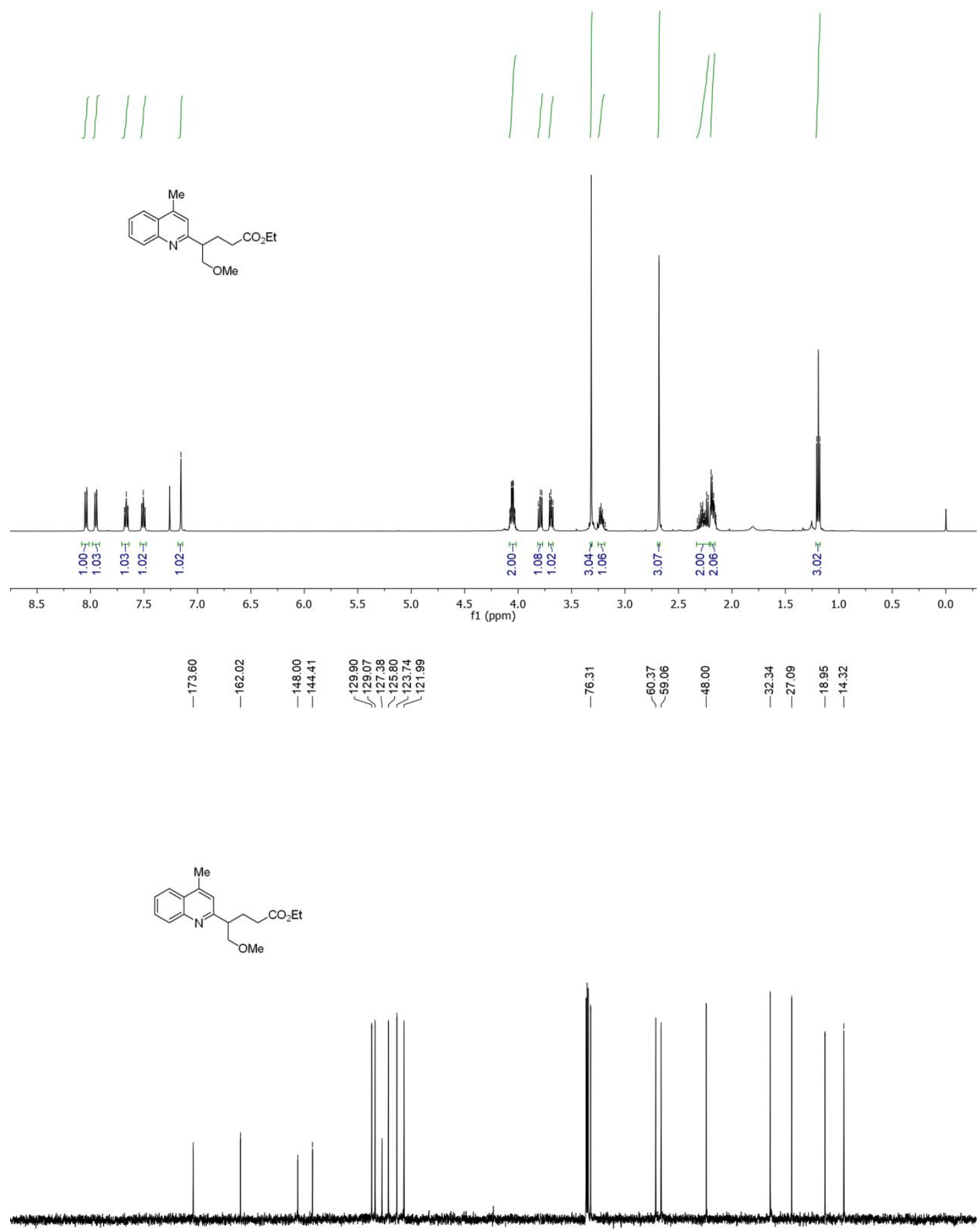

$\begin{array}{lllllllllllllllllllllllllll}1 & 1 & 200 & 190 & 180 & 170 & 160 & 150 & 140 & 130 & 120 & 110 & 100 & 90 & 80 & 70 & 60 & 50 & 40 & 30 & 20 & 10 & 0 & -10\end{array}$ 
${ }^{1} \mathrm{H}$ NMR (400 MHz, $\left.\mathrm{CDCl}_{3}\right)$ and ${ }^{13} \mathrm{C}$ NMR (125 $\left.\mathrm{MHz}, \mathrm{CDCl}_{3}\right)$ spectra for $4 \mathrm{~g}$

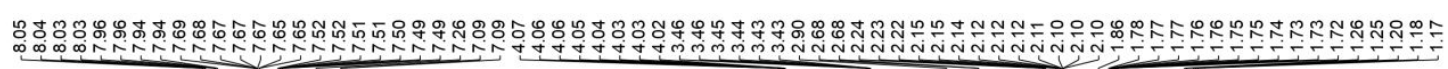

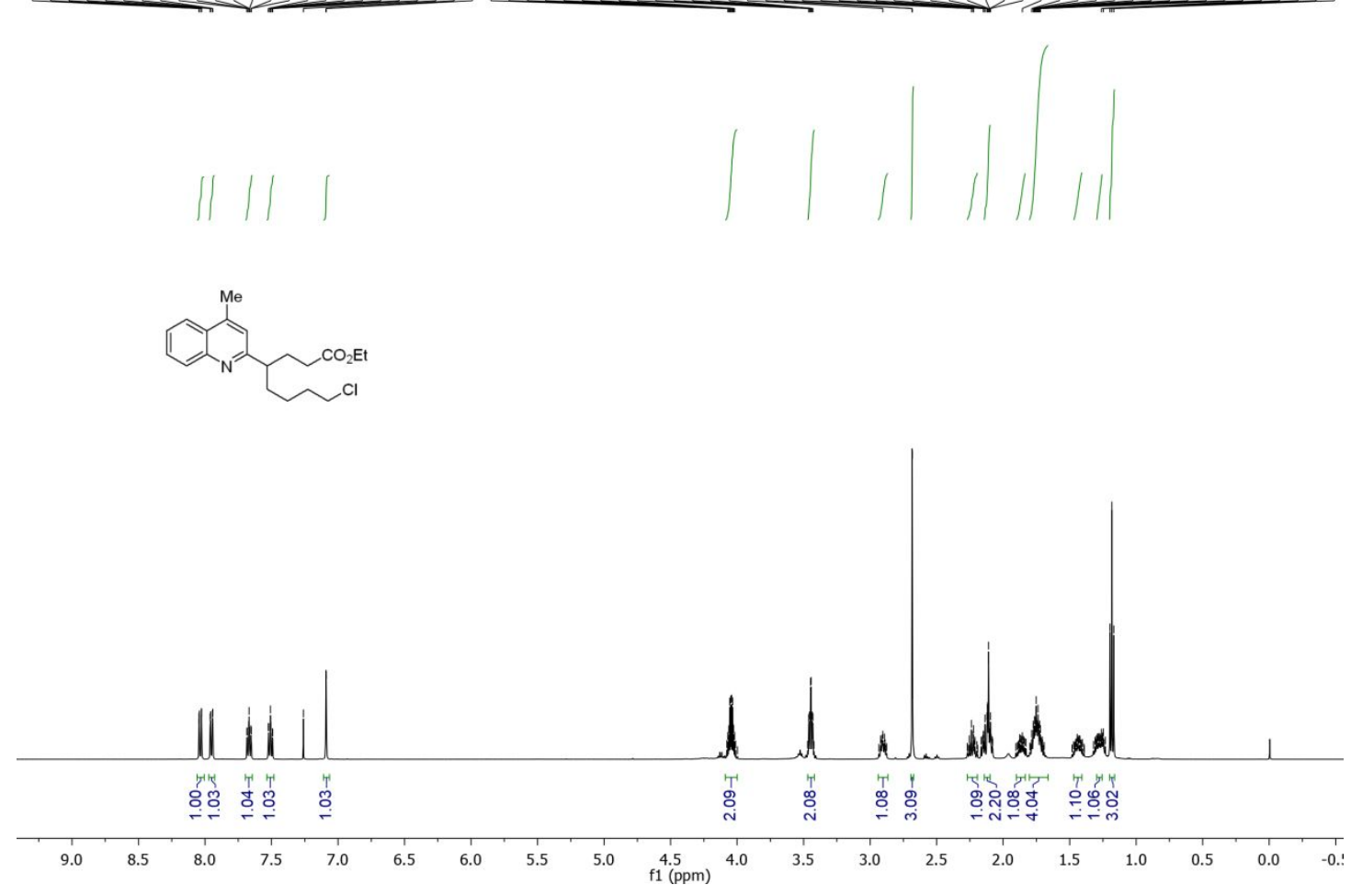

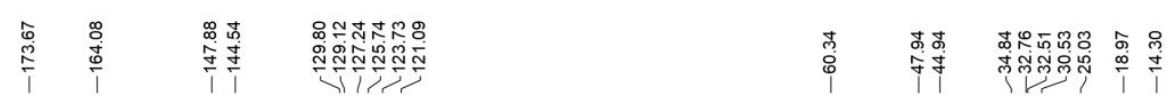
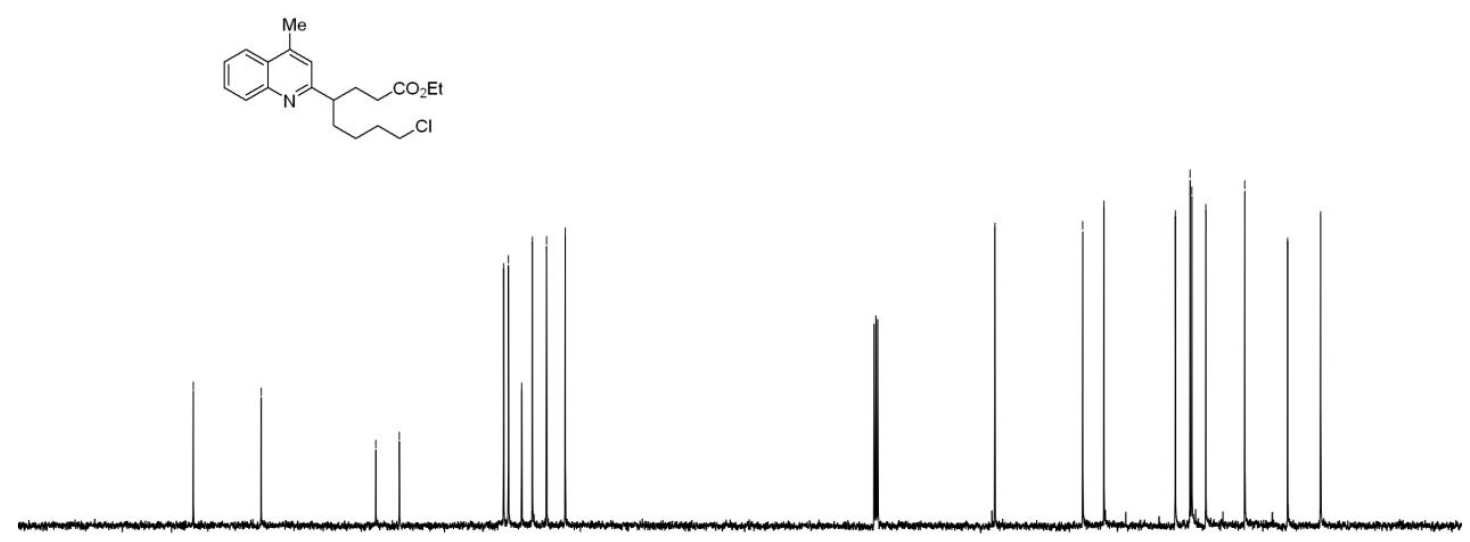

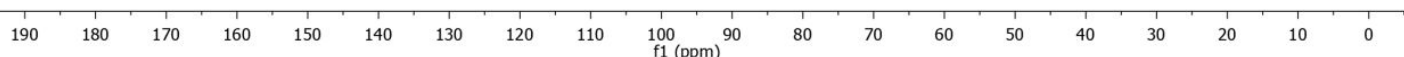


${ }^{1} \mathrm{H}$ NMR (400 MHz, $\left.\mathrm{CDCl}_{3}\right)$ and ${ }^{13} \mathrm{C}$ NMR (125 MHz, $\left.\mathrm{CDCl}_{3}\right)$ spectra for $4 \mathrm{~h}$

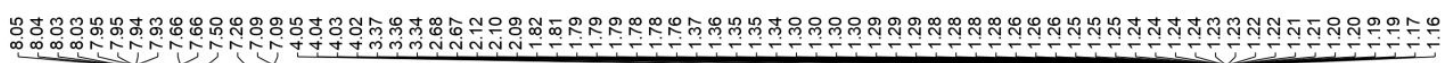

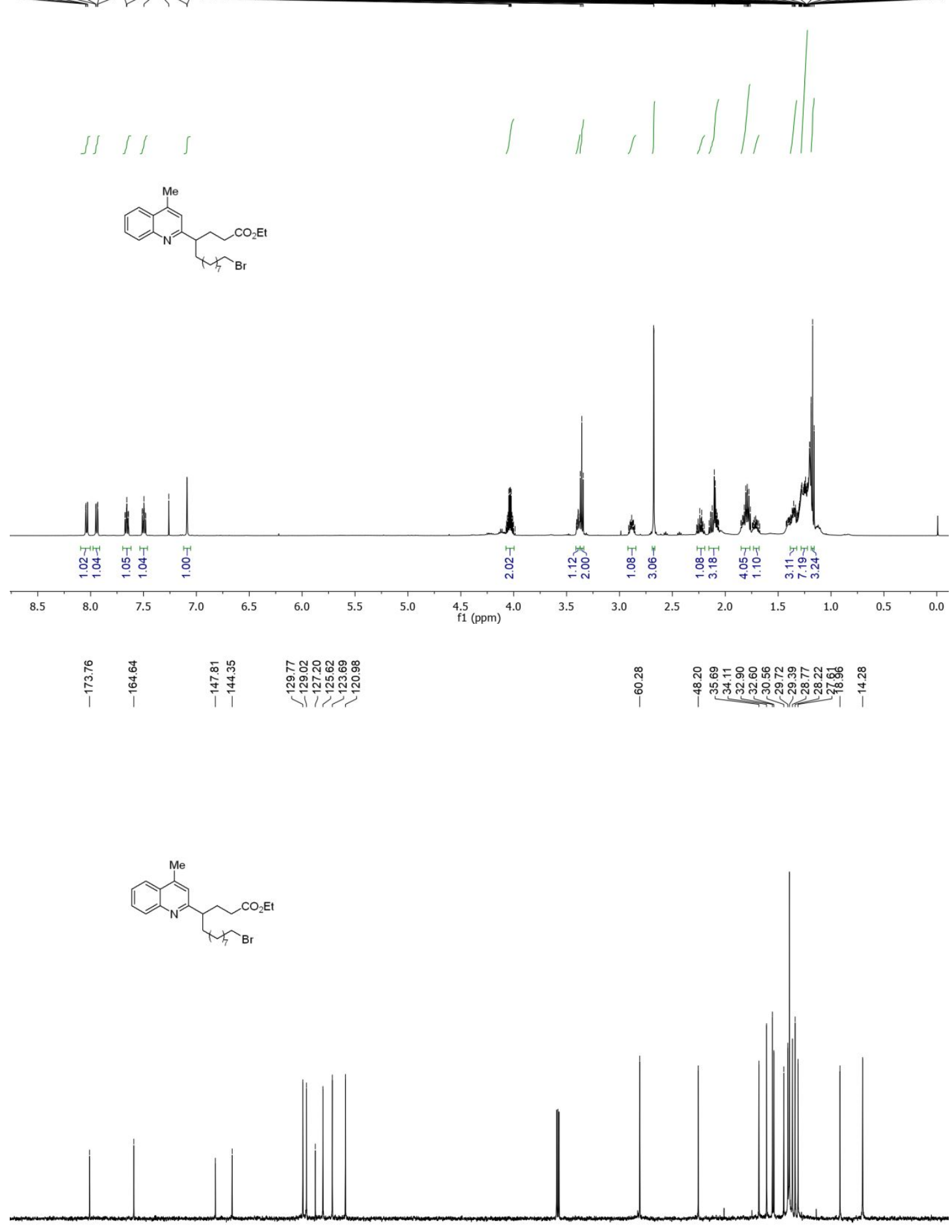

\begin{tabular}{llllllllllllllllllll}
\hline & 180 & 170 & 160 & 150 & 140 & 130 & 120 & 110 & 100 & 90 & 80 & 70 & 60 & 50 & 40 & 30 & 20 & 10 & 0
\end{tabular} 
${ }^{1} \mathrm{H}$ NMR (400 MHz, $\left.\mathrm{CDCl}_{3}\right)$ and ${ }^{13} \mathrm{C}$ NMR (125 MHz, $\left.\mathrm{CDCl}_{3}\right)$ spectra for $4 \mathrm{i}$

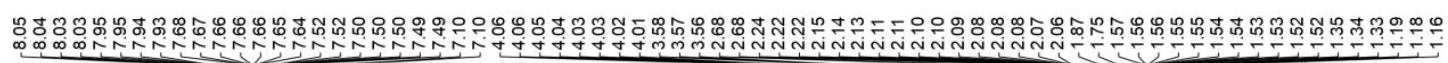
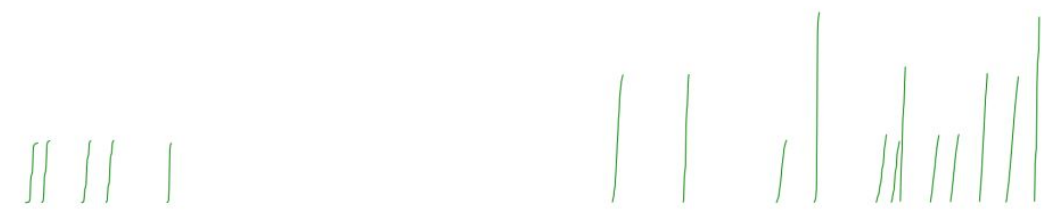

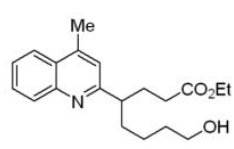

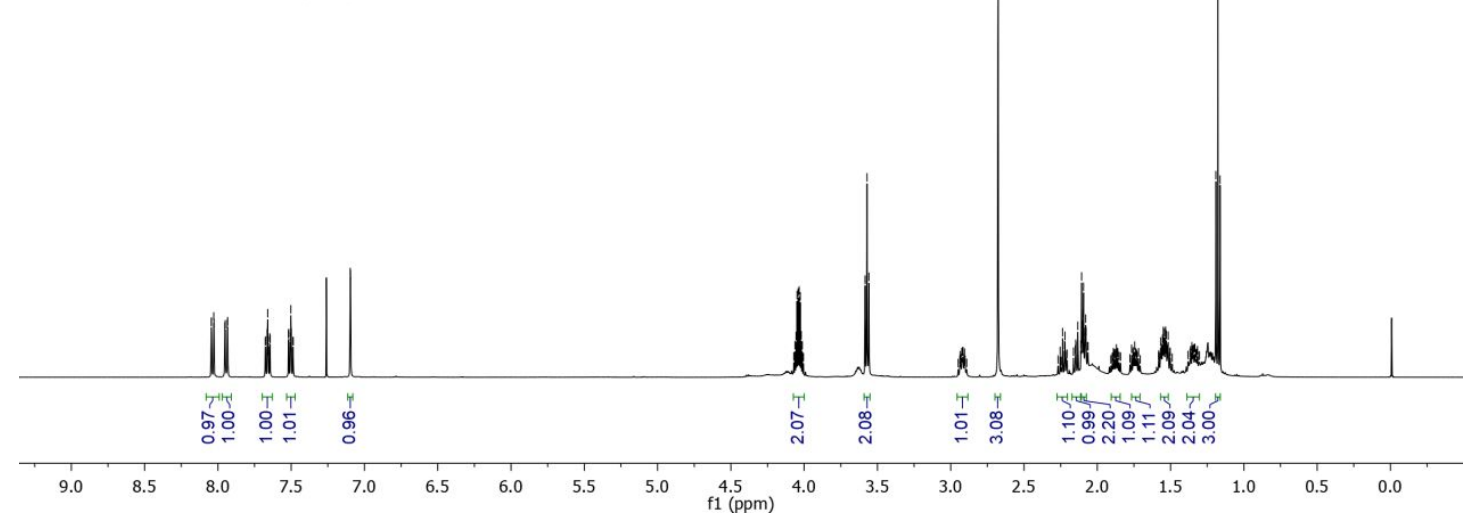

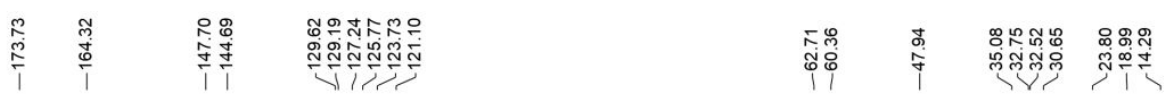
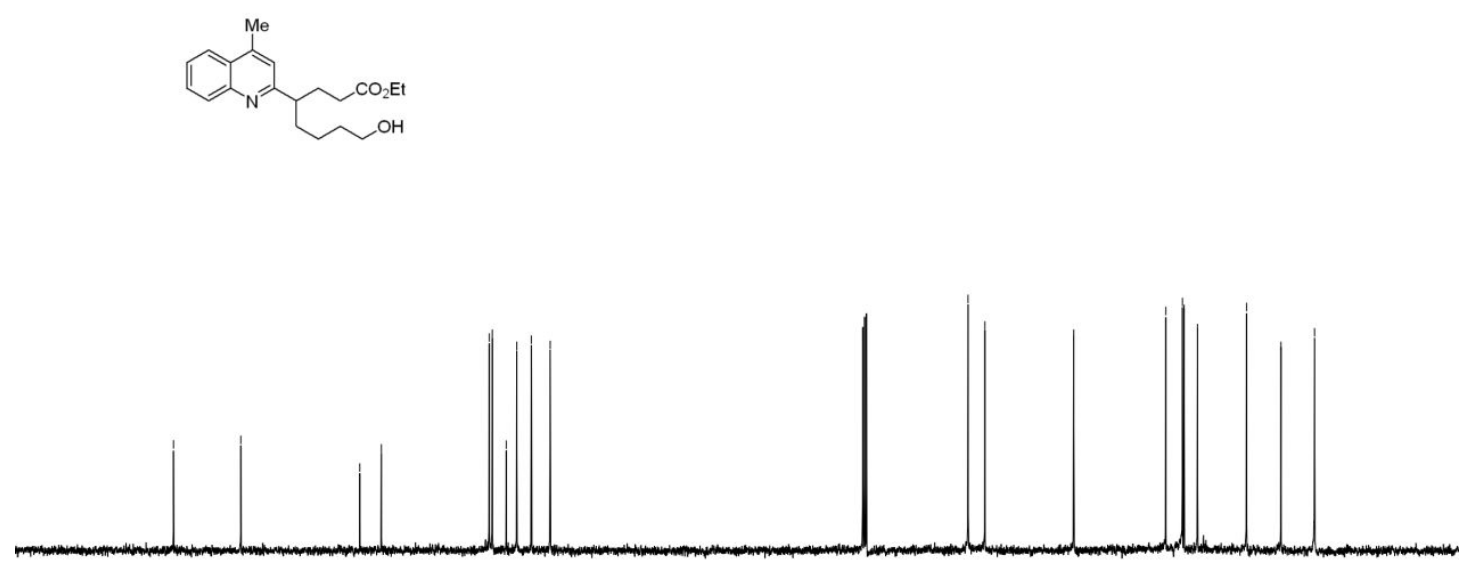
${ }^{1} \mathrm{H}$ NMR (400 MHz, $\left.\mathrm{CDCl}_{3}\right)$ and ${ }^{13} \mathrm{C}$ NMR (125 $\left.\mathrm{MHz}, \mathrm{CDCl}_{3}\right)$ spectra for $4 \mathrm{j}$

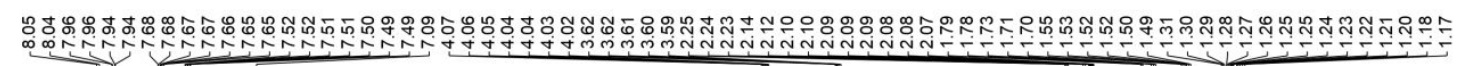
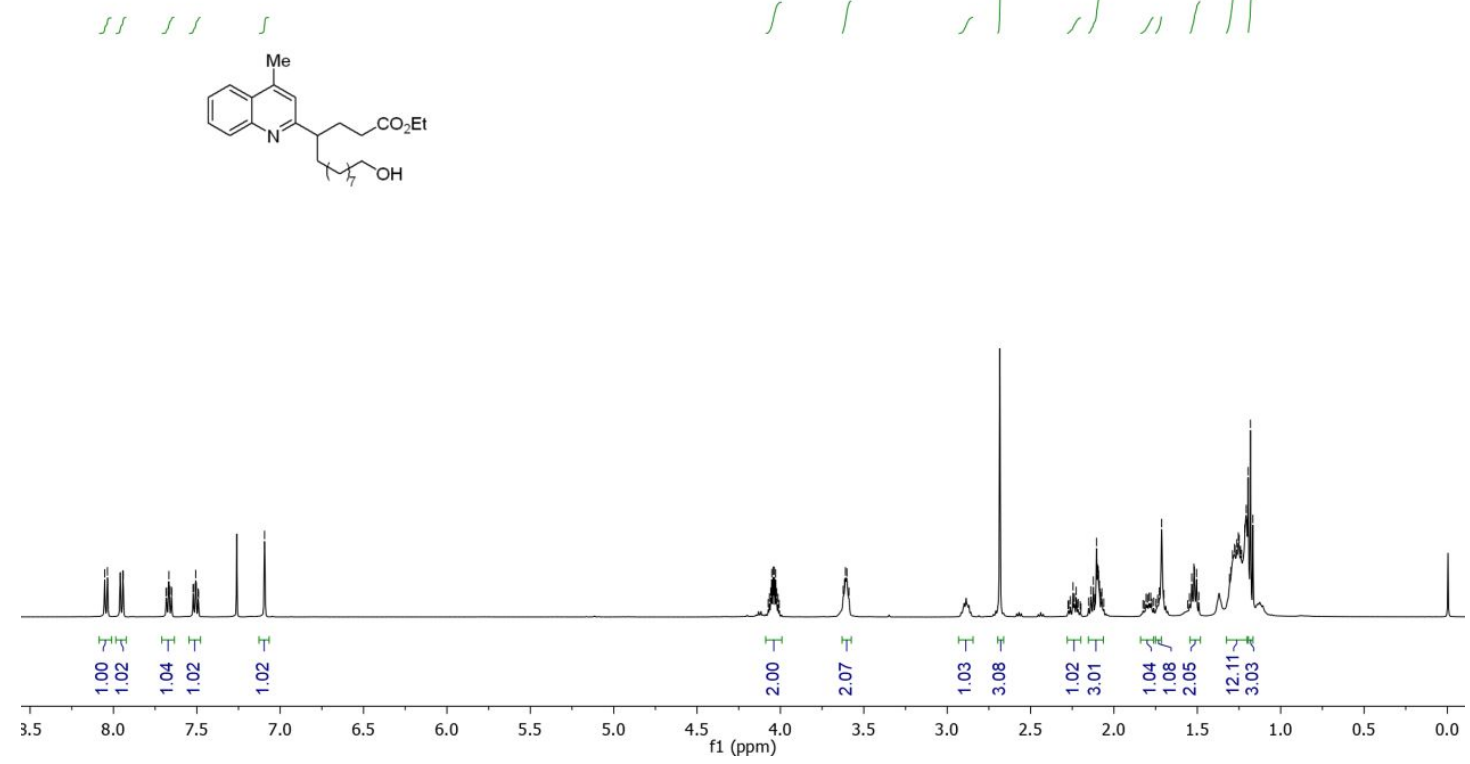

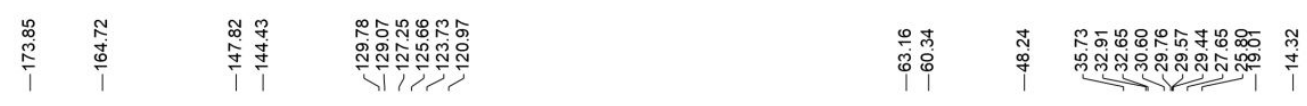

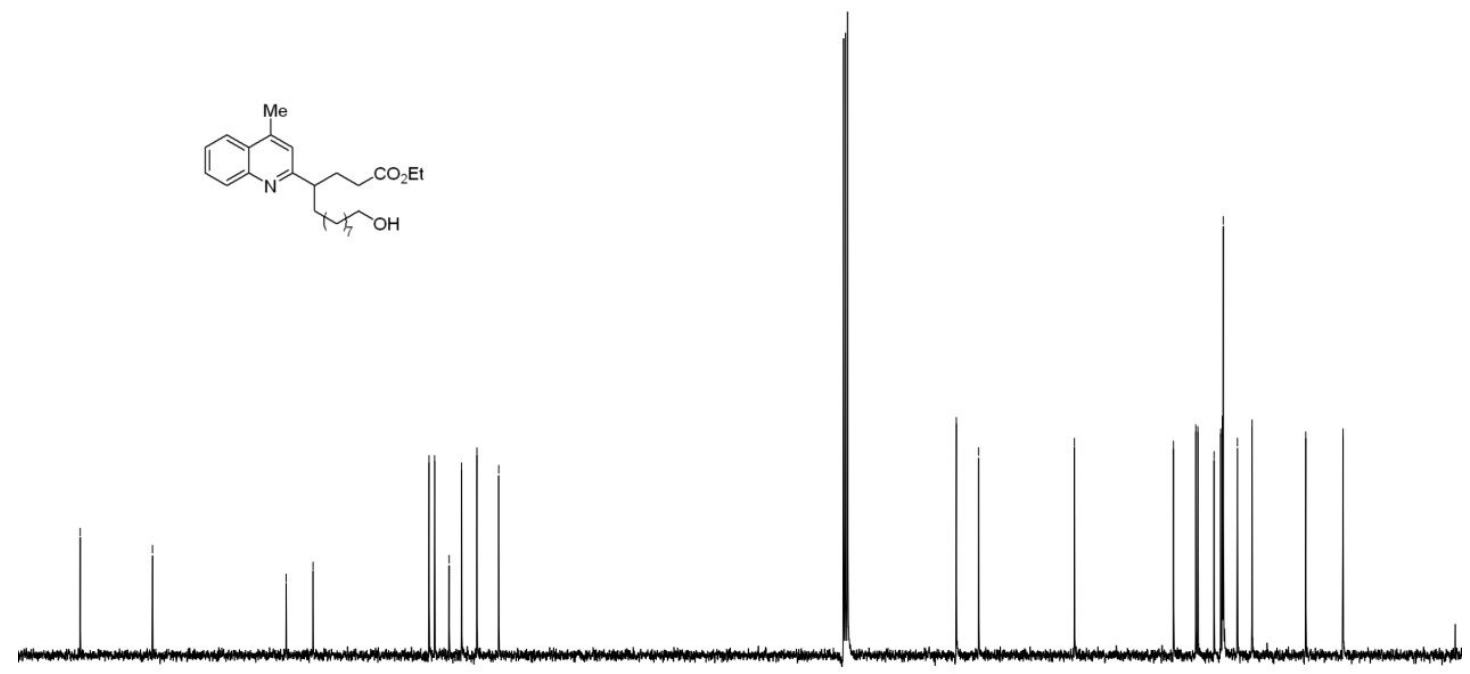

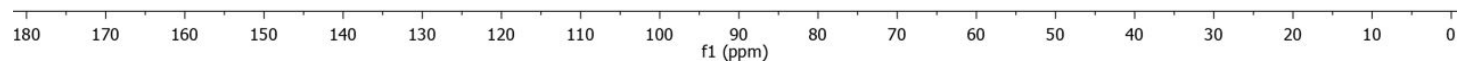


${ }^{1} \mathrm{H}$ NMR (400 $\left.\mathrm{MHz}, \mathrm{CDCl}_{3}\right)$ and ${ }^{13} \mathrm{C}$ NMR (125 $\left.\mathrm{MHz}, \mathrm{CDCl}_{3}\right)$ spectra for $4 \mathrm{k}$

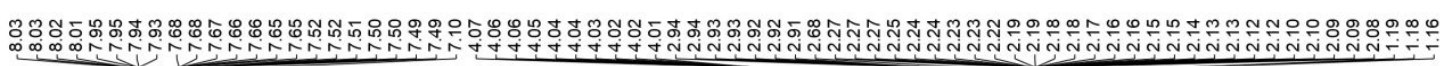

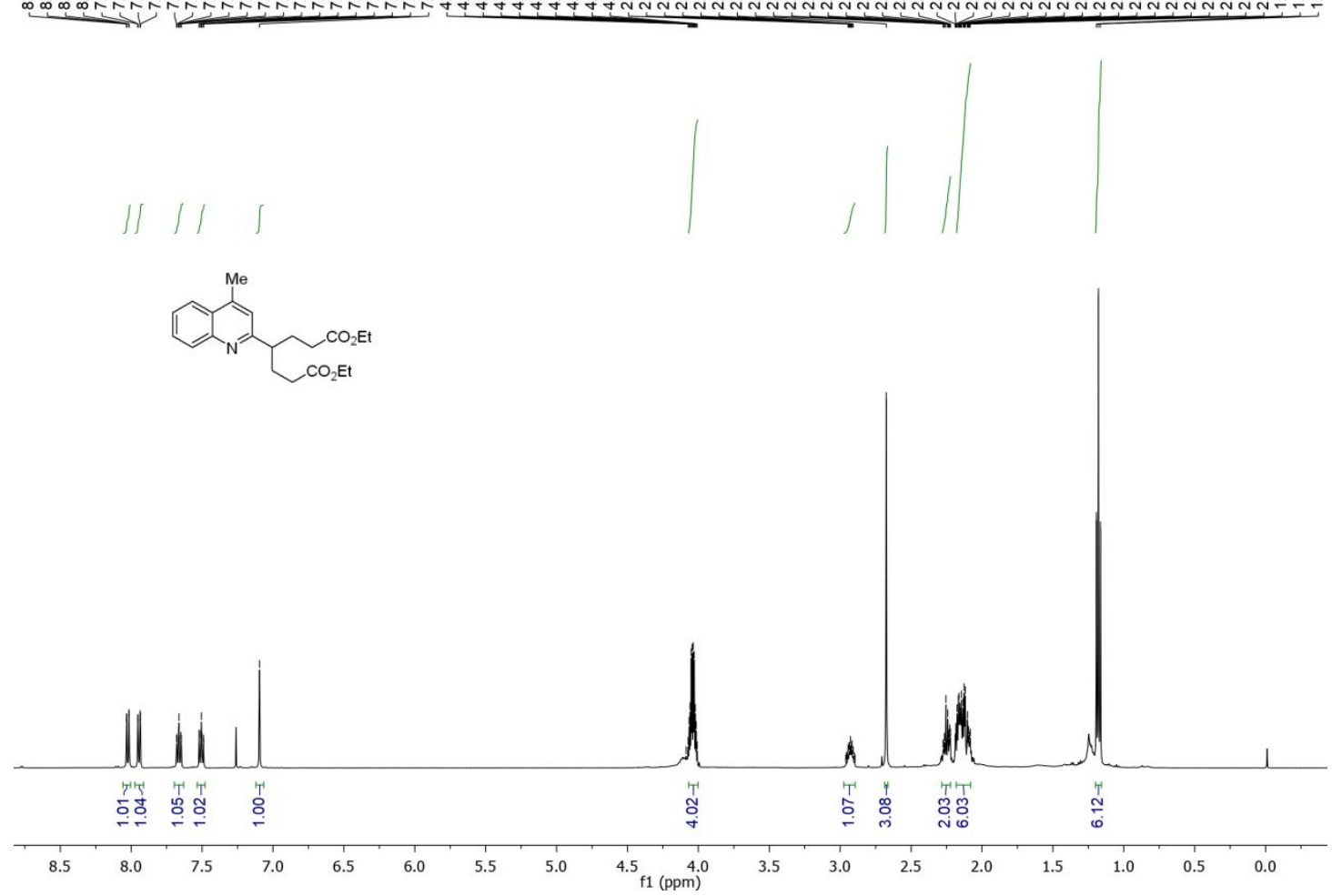

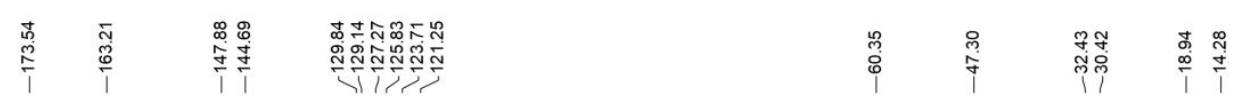
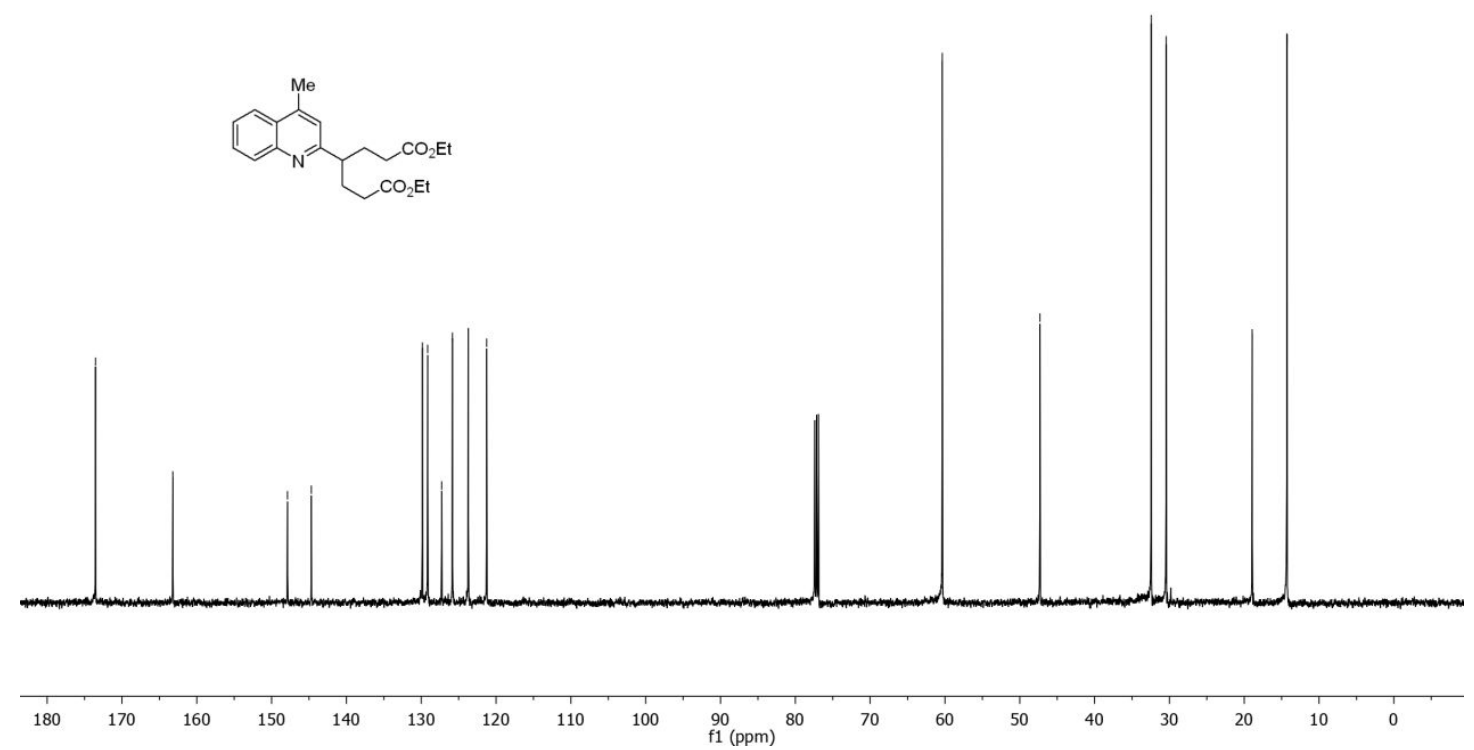
${ }^{1} \mathrm{H}$ NMR (400 MHz, $\left.\mathrm{CDCl}_{3}\right)$ and ${ }^{13} \mathrm{C}$ NMR (125 $\left.\mathrm{MHz}, \mathrm{CDCl}_{3}\right)$ spectra for 41

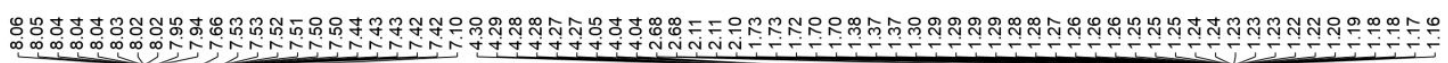

$1,, 1 / s$

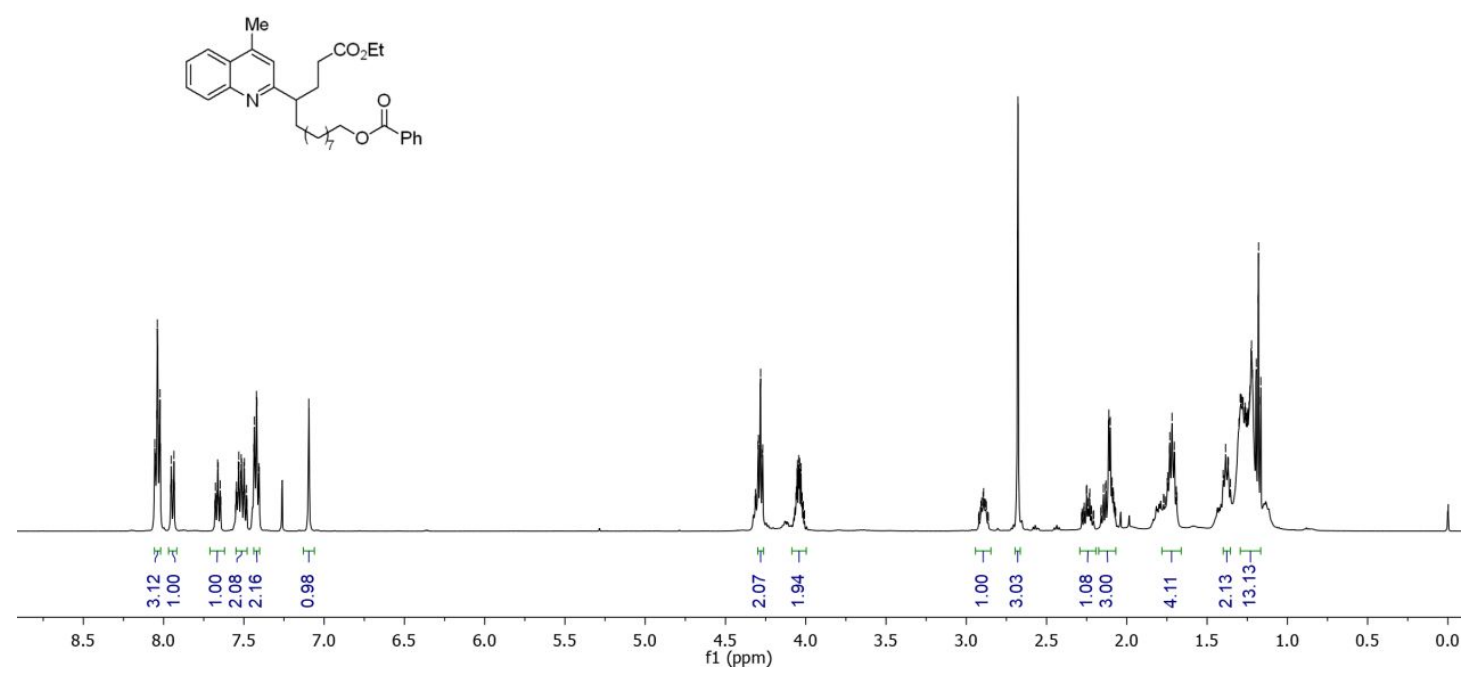

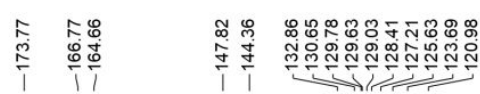

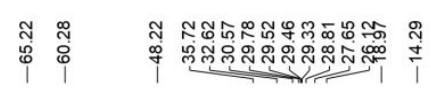

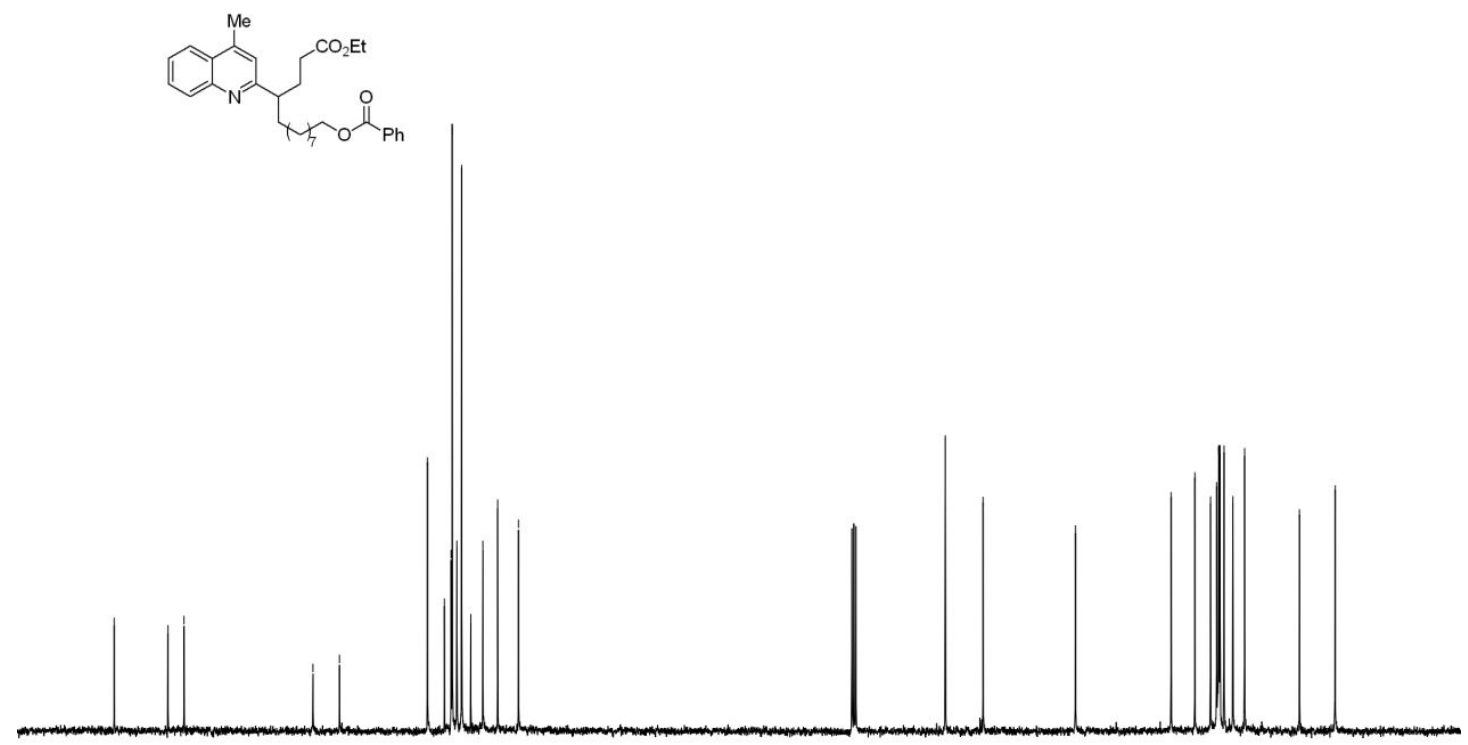

$\begin{array}{llllllllllllllllll}1 & 1 & 1 & 1 & 1 & 1 & 1 & 1 & 1 & 1 & 1 & 1 & 1 & 1 & 1 & 1 & 1 & 1 \\ \mathrm{f} 1(\mathrm{ppm}) & 80 & 70 & 60 & 50 & 40 & 30 & 20 & 10 & 0\end{array}$ 
${ }^{1} \mathrm{H}$ NMR (400 MHz, $\left.\mathrm{CDCl}_{3}\right)$ and ${ }^{13} \mathrm{C}$ NMR (125 $\left.\mathrm{MHz}, \mathrm{CDCl}_{3}\right)$ spectra for $4 \mathrm{~m}$

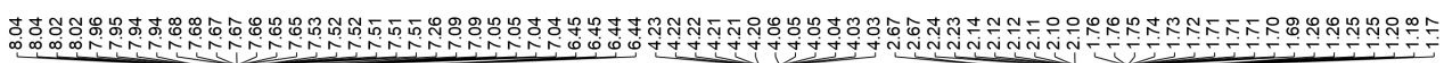
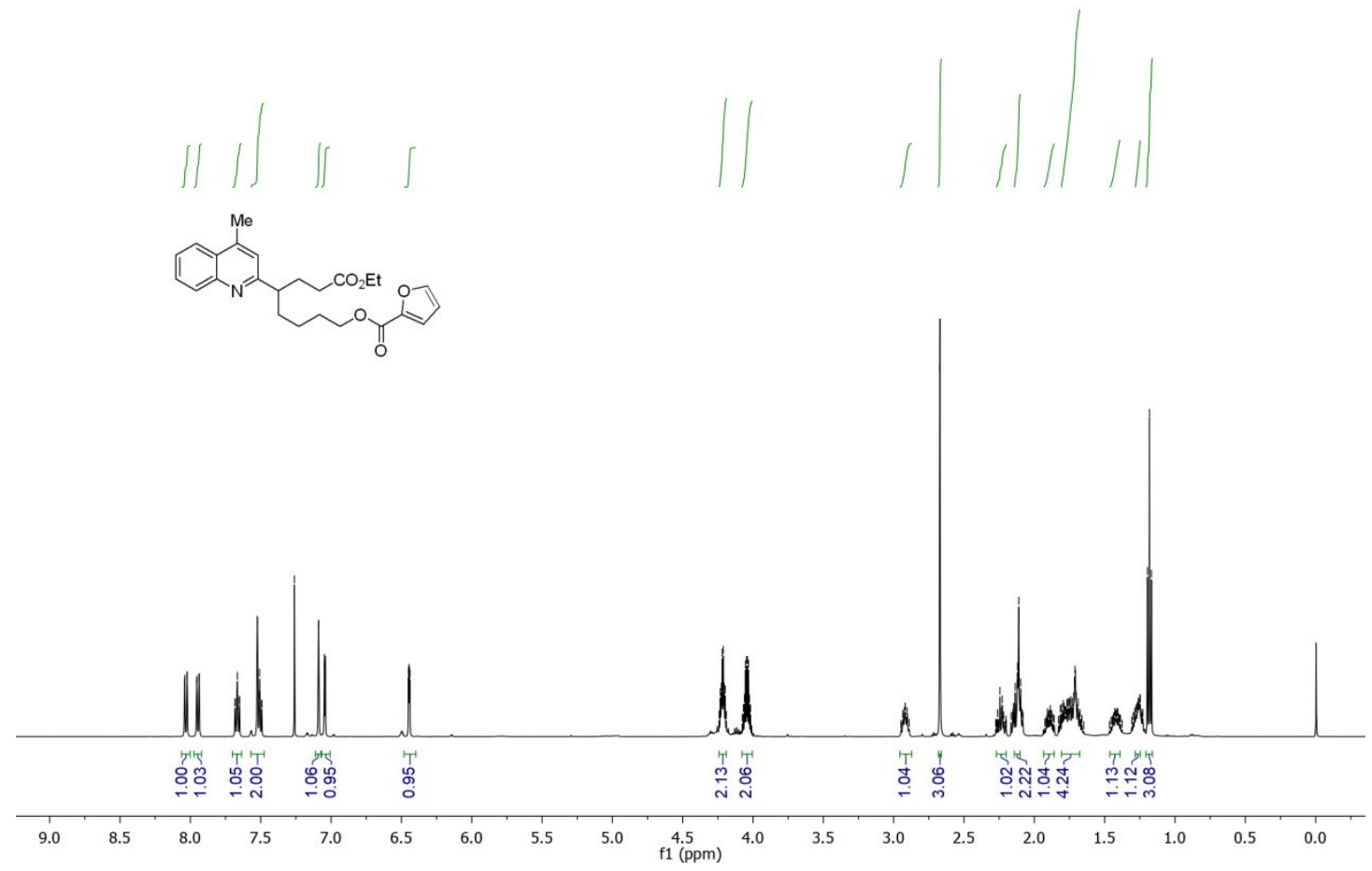

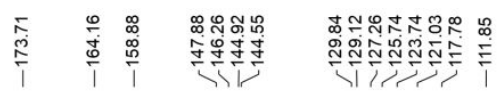

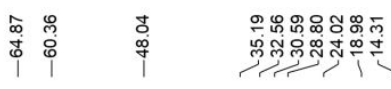
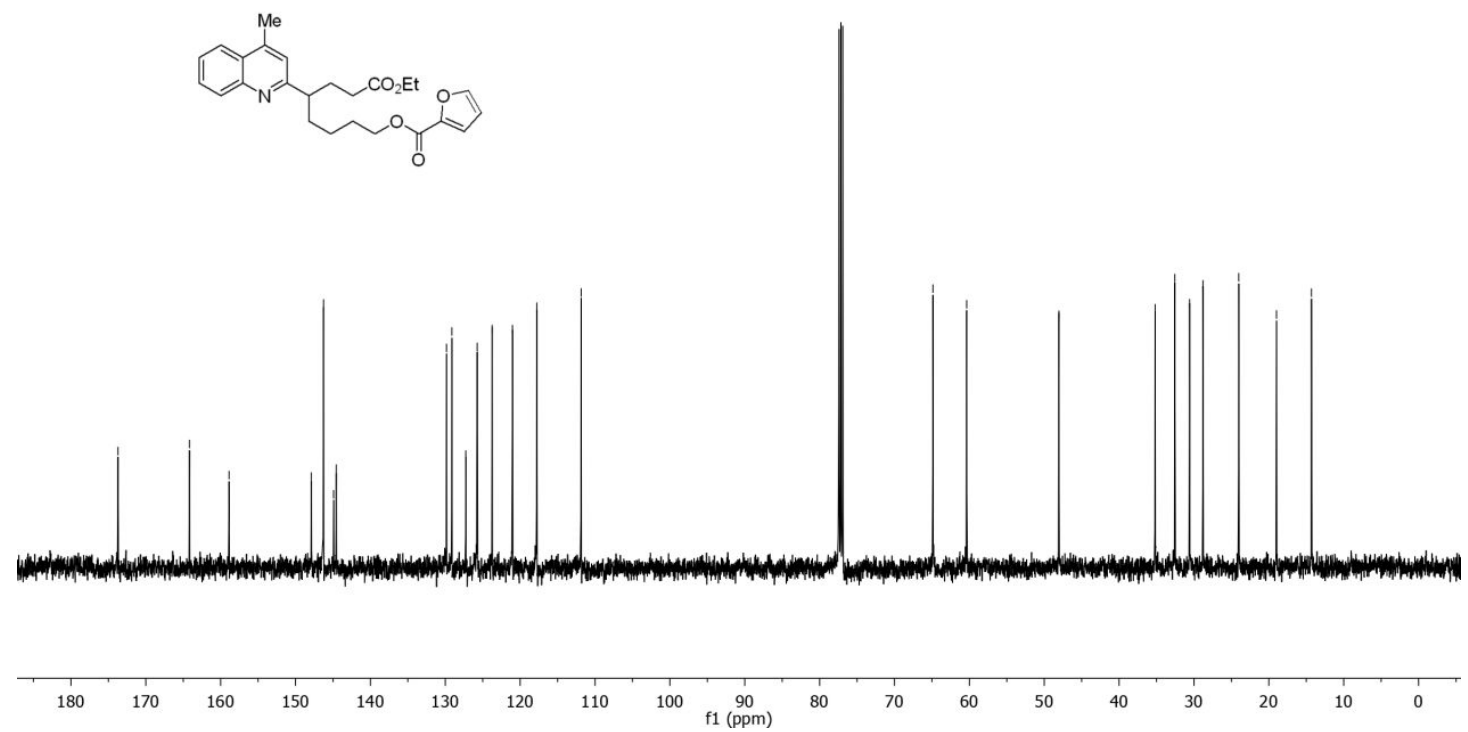
${ }^{1} \mathrm{H}$ NMR (400 MHz, $\left.\mathrm{CDCl}_{3}\right)$ and ${ }^{13} \mathrm{C}$ NMR (125 $\left.\mathrm{MHz}, \mathrm{CDCl}_{3}\right)$ spectra for 4n

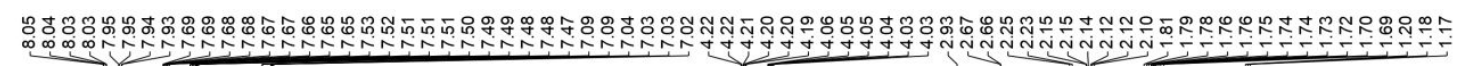

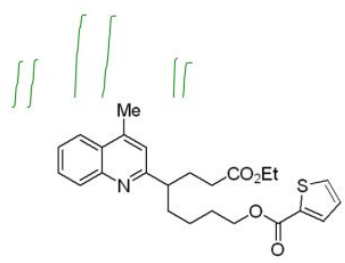
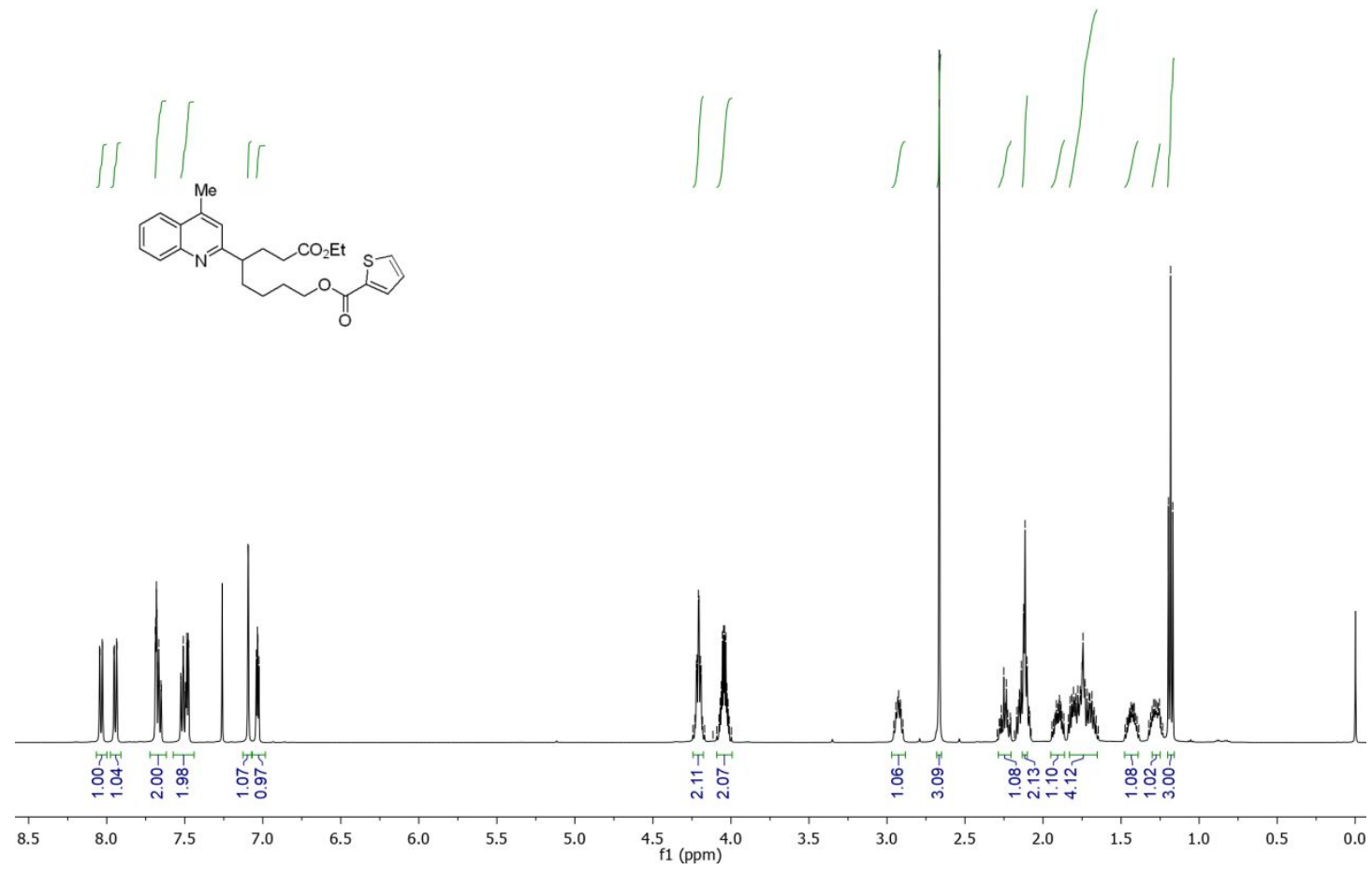

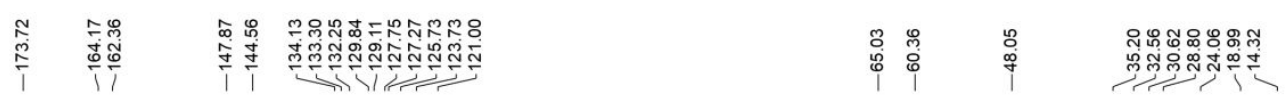

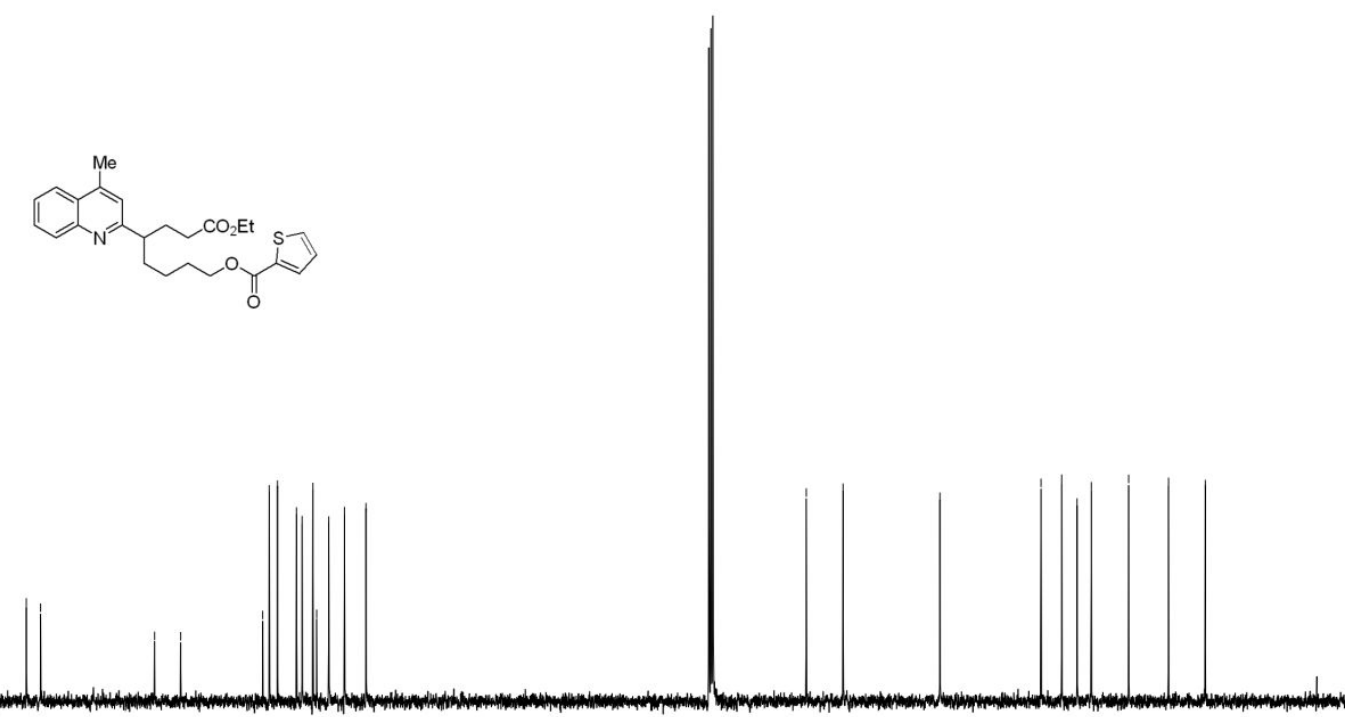

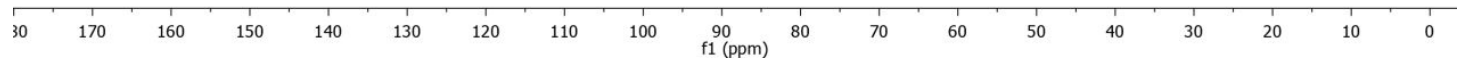


${ }^{1} \mathrm{H}$ NMR (400 MHz, $\left.\mathrm{CDCl}_{3}\right)$ and ${ }^{13} \mathrm{C}$ NMR (125 $\left.\mathrm{MHz}, \mathrm{CDCl}_{3}\right)$ spectra for 40

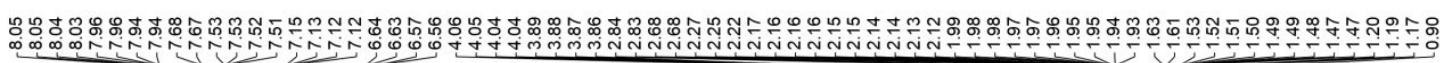
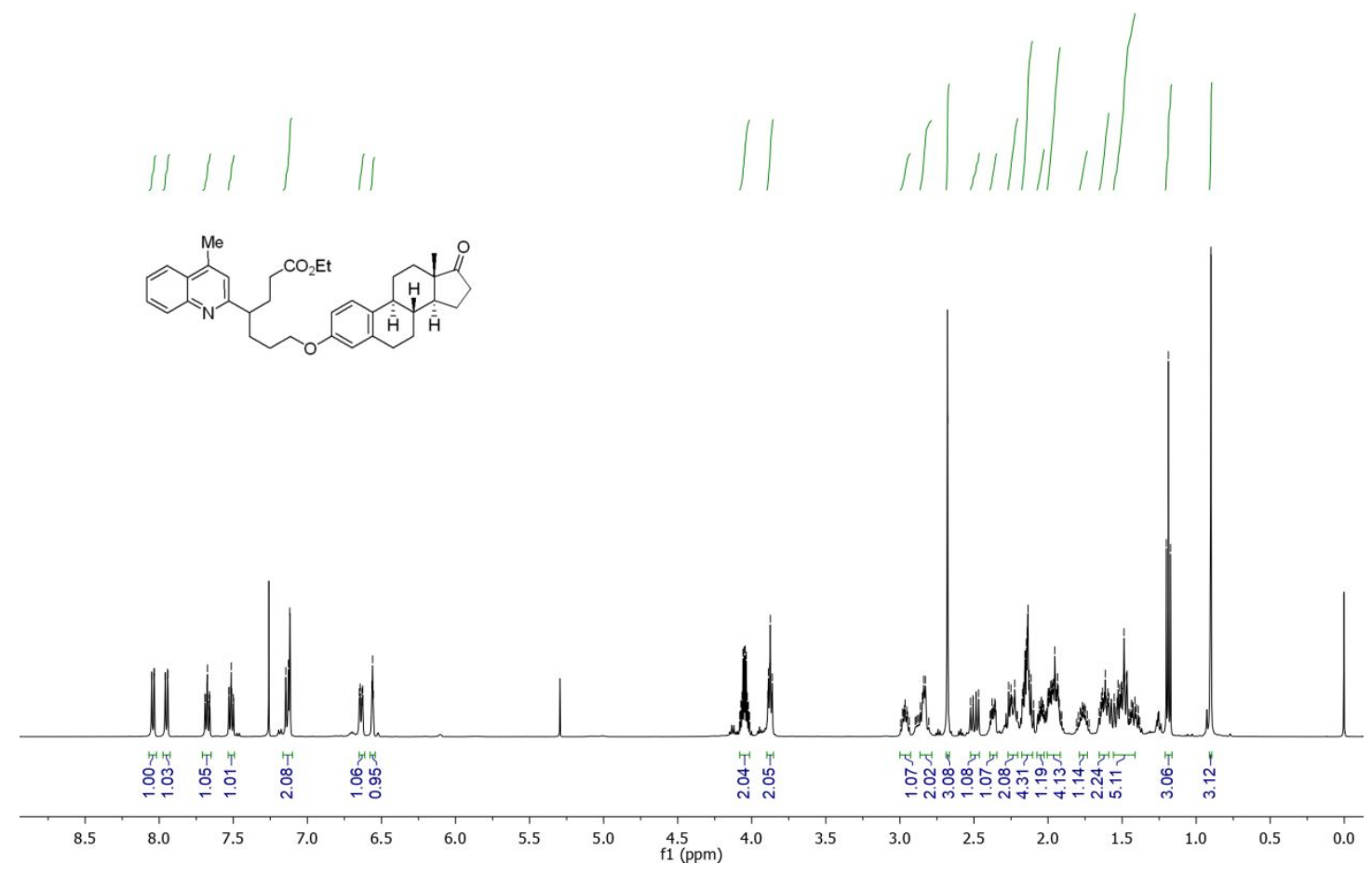

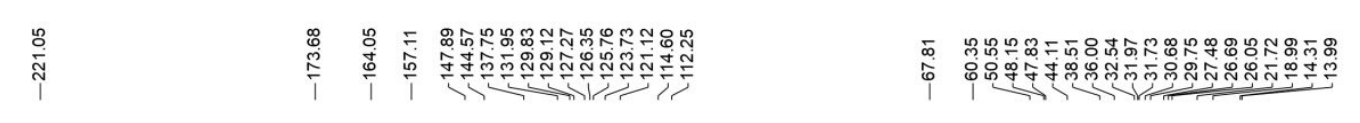

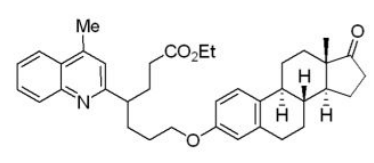

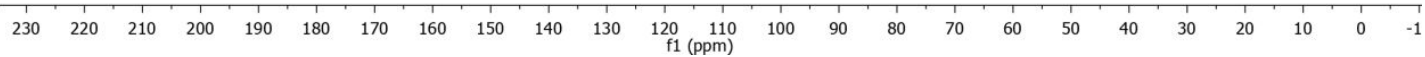


${ }^{1} \mathrm{H}$ NMR (400 MHz, $\left.\mathrm{CDCl}_{3}\right)$ and ${ }^{13} \mathrm{C}$ NMR (125 MHz, $\left.\mathrm{CDCl}_{3}\right)$ spectra for $4 \mathrm{p}$

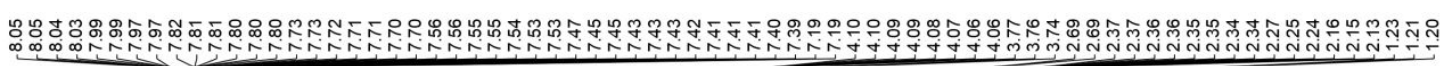
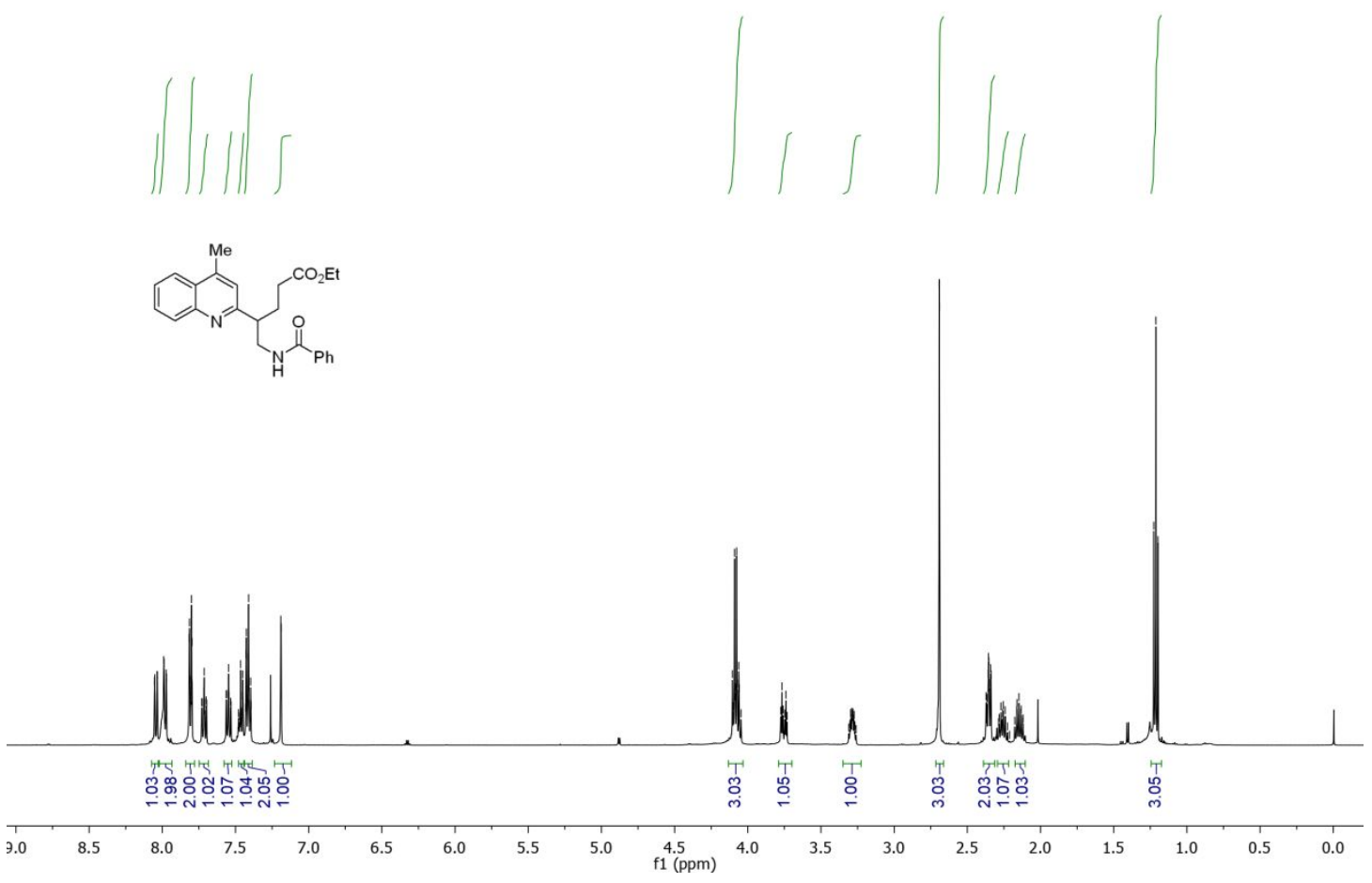

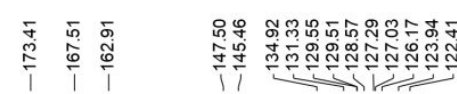

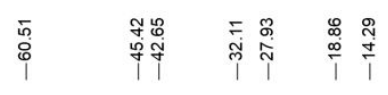

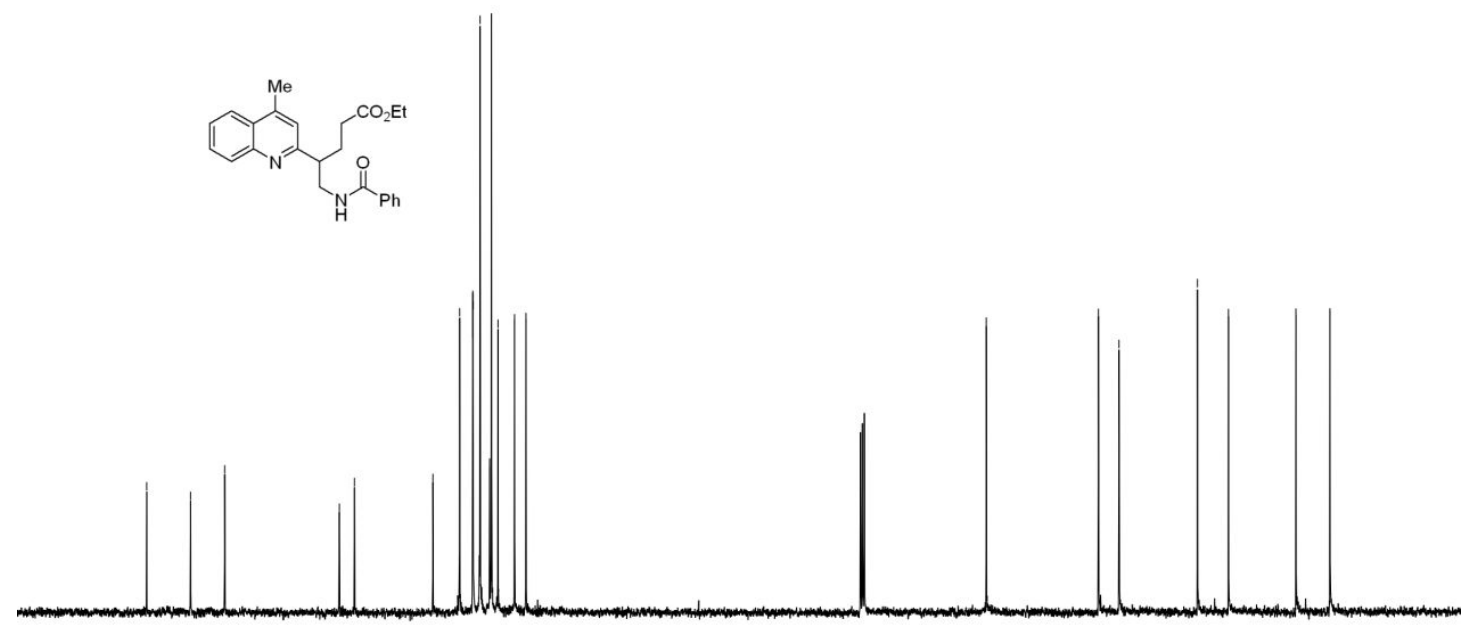

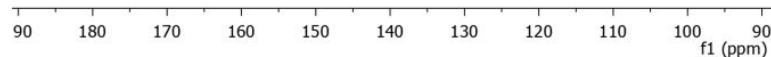


${ }^{1} \mathrm{H}$ NMR (400 $\left.\mathrm{MHz}, \mathrm{CDCl}_{3}\right)$ and ${ }^{13} \mathrm{C}$ NMR (125 $\left.\mathrm{MHz}, \mathrm{CDCl}_{3}\right)$ spectra for $4 \mathrm{q}$

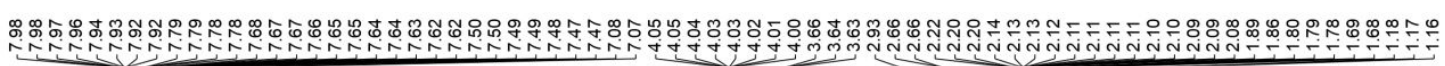
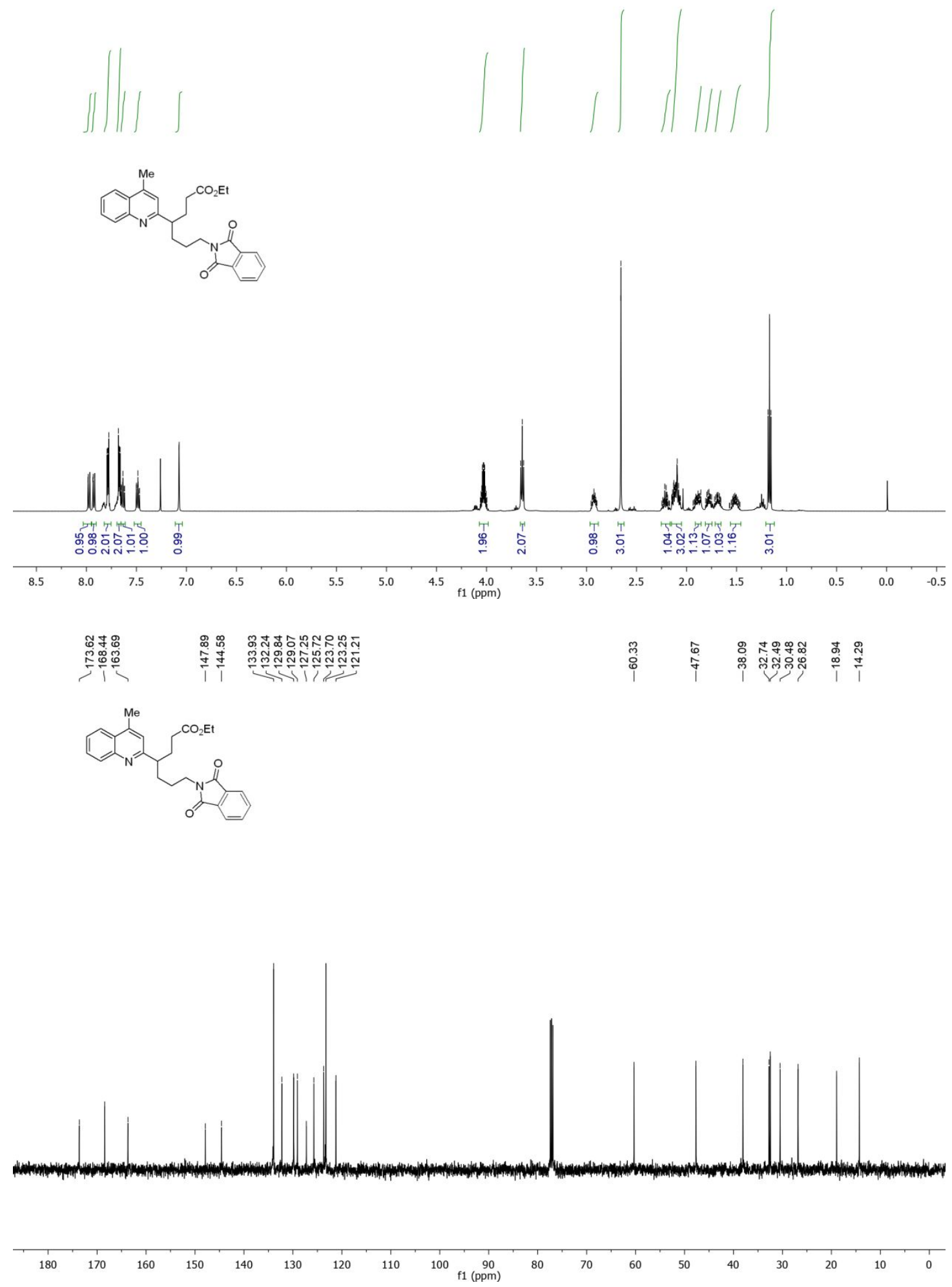
${ }^{1} \mathrm{H}$ NMR (400 MHz, $\left.\mathrm{CDCl}_{3}\right)$ and ${ }^{13} \mathrm{C}$ NMR (125 MHz, $\left.\mathrm{CDCl}_{3}\right)$ spectra for $7 \mathrm{a}$

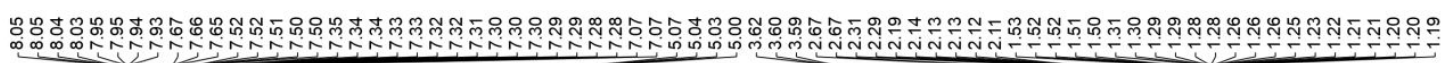
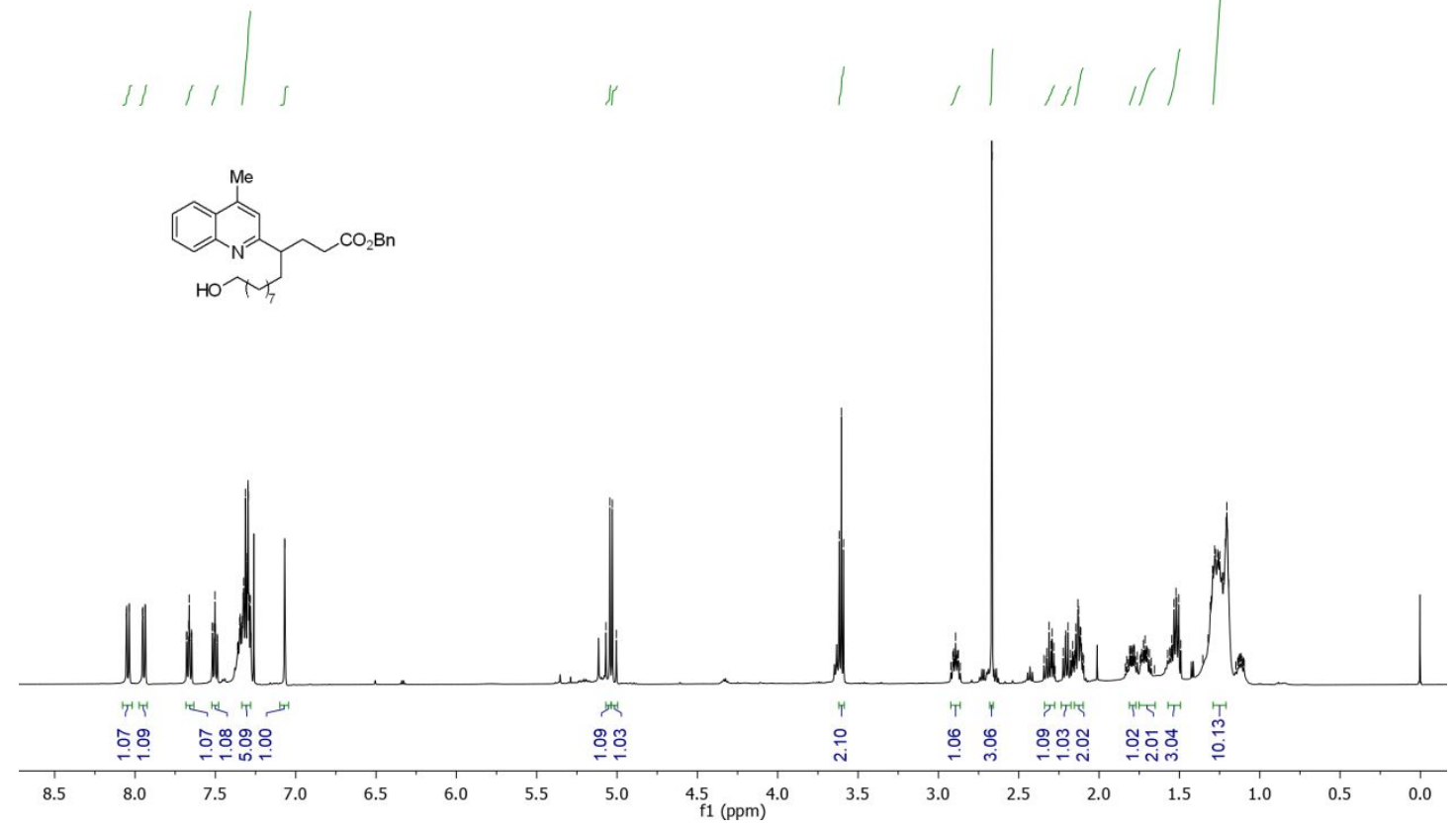

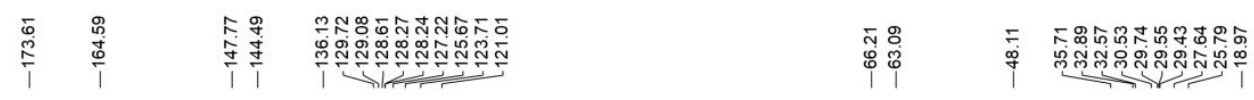

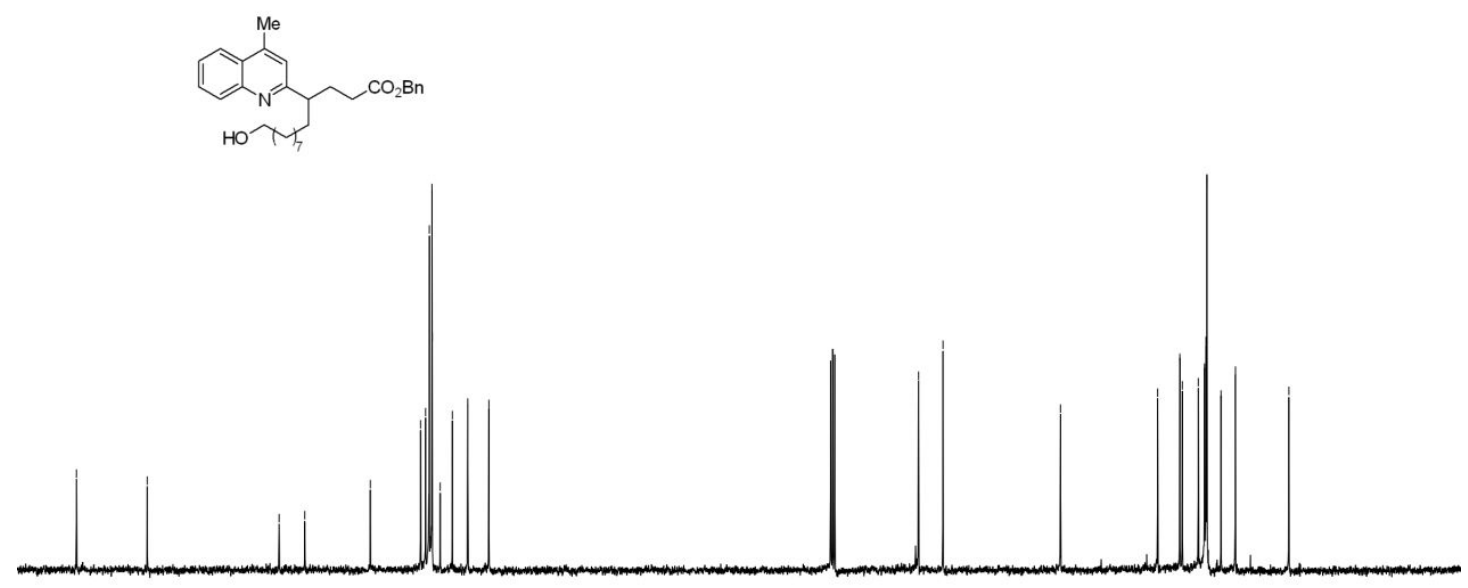

$\begin{array}{lllllllllllllllllll}1 & 1 \\ 180 & 170 & 160 & 150 & 140 & 130 & 120 & 110 & 100 & \underset{f}{90} & 80 & 70 & 60 & 50 & 40 & 30 & 20 & 10 & 0\end{array}$ 
${ }^{1} \mathrm{H}$ NMR (400 MHz, $\left.\mathrm{CDCl}_{3}\right)$ and ${ }^{13} \mathrm{C}$ NMR (125 $\left.\mathrm{MHz}, \mathrm{CDCl}_{3}\right)$ spectra for $7 \mathrm{~b}$

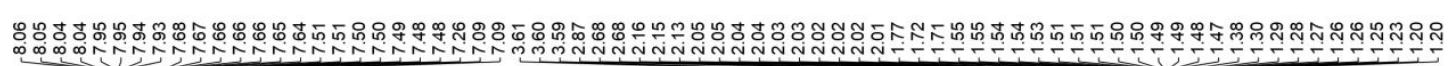

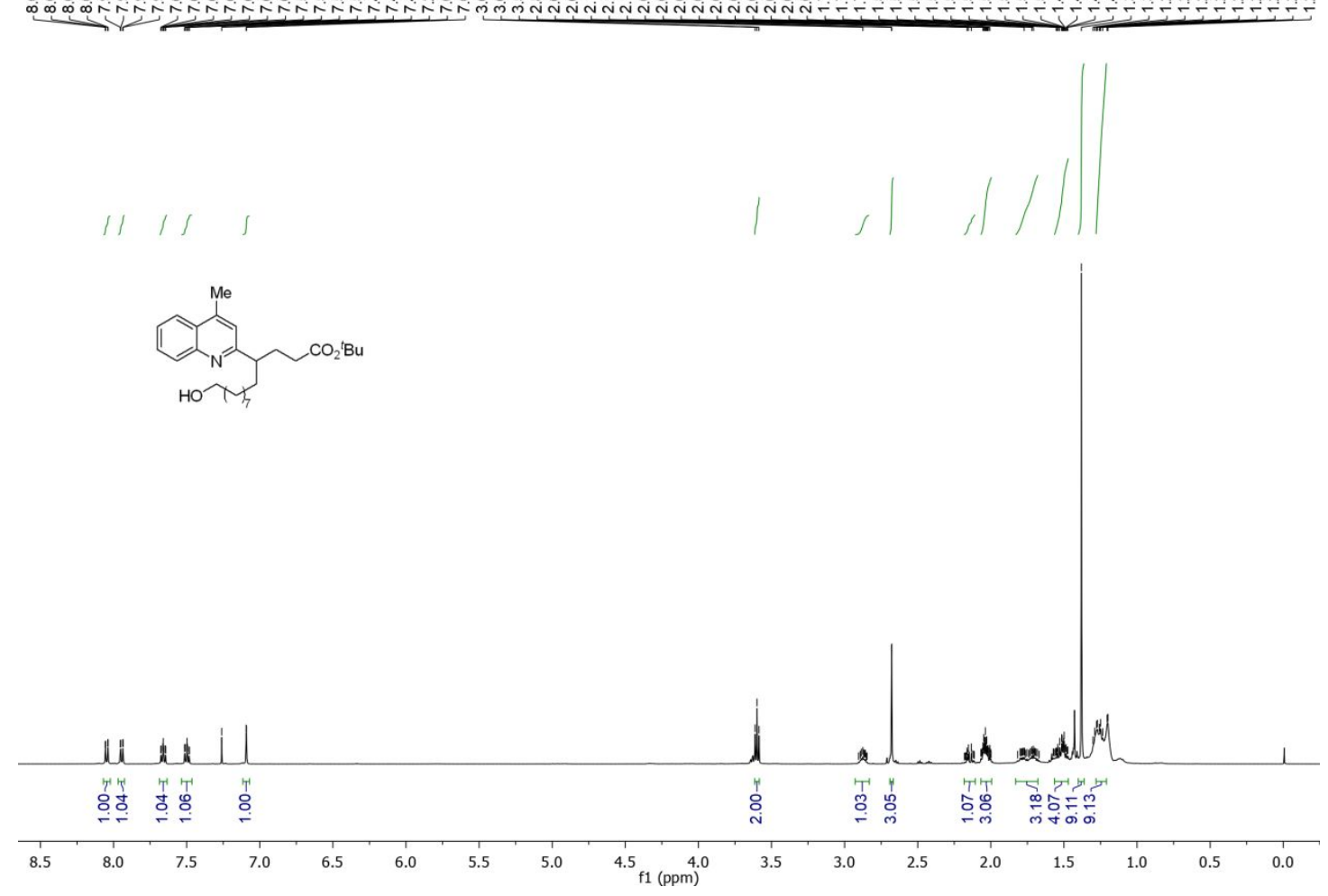

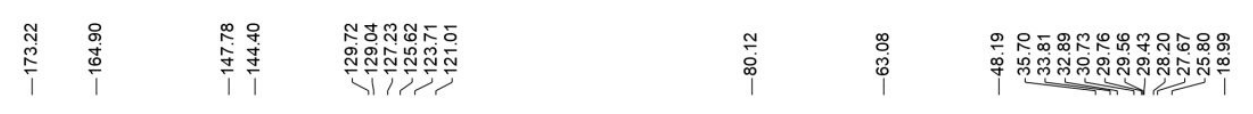
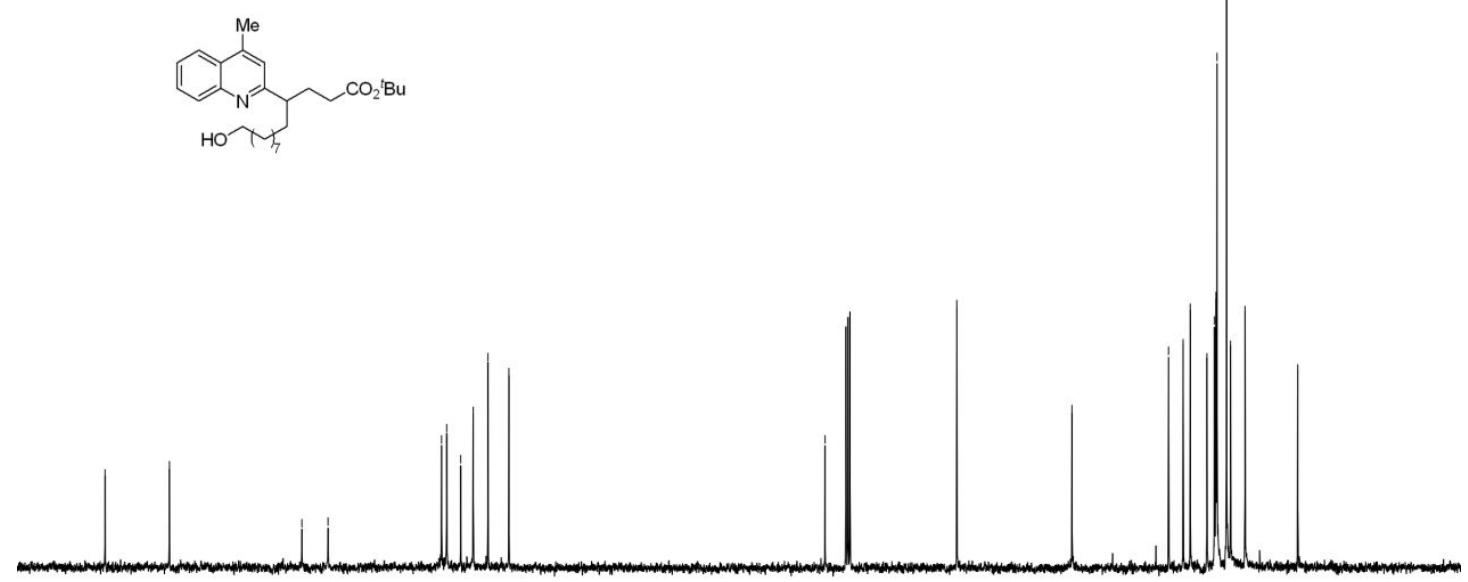

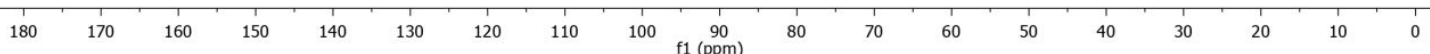


${ }^{1} \mathrm{H}$ NMR (400 MHz, $\left.\mathrm{CDCl}_{3}\right)$ and ${ }^{13} \mathrm{C}$ NMR (125 MHz, $\left.\mathrm{CDCl}_{3}\right)$ spectra for 7c

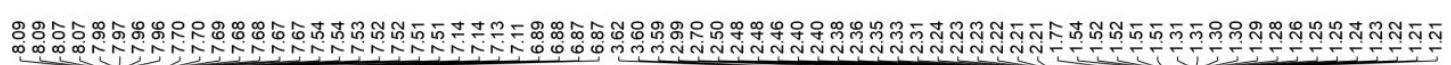
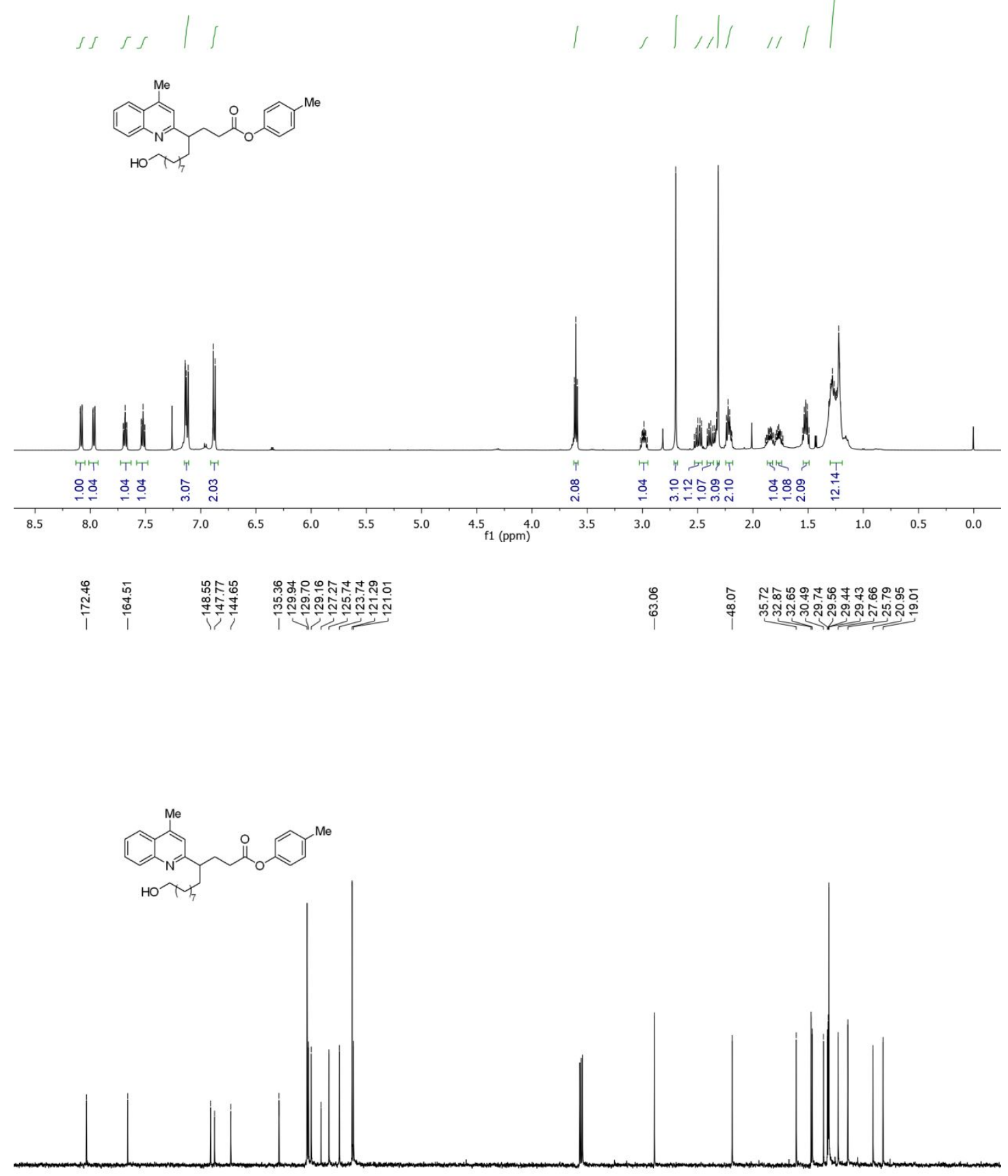

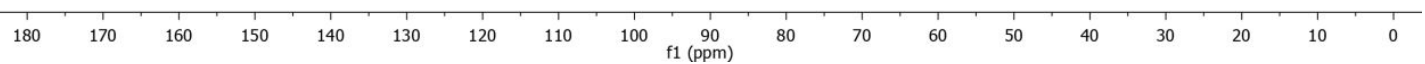


${ }^{1} \mathrm{H}$ NMR (400 MHz, $\left.\mathrm{CDCl}_{3}\right)$ and ${ }^{13} \mathrm{C}$ NMR (125 MHz, $\left.\mathrm{CDCl}_{3}\right)$ spectra for 7d

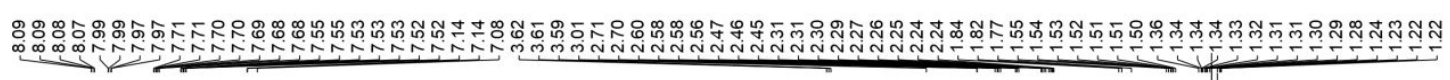

11,11

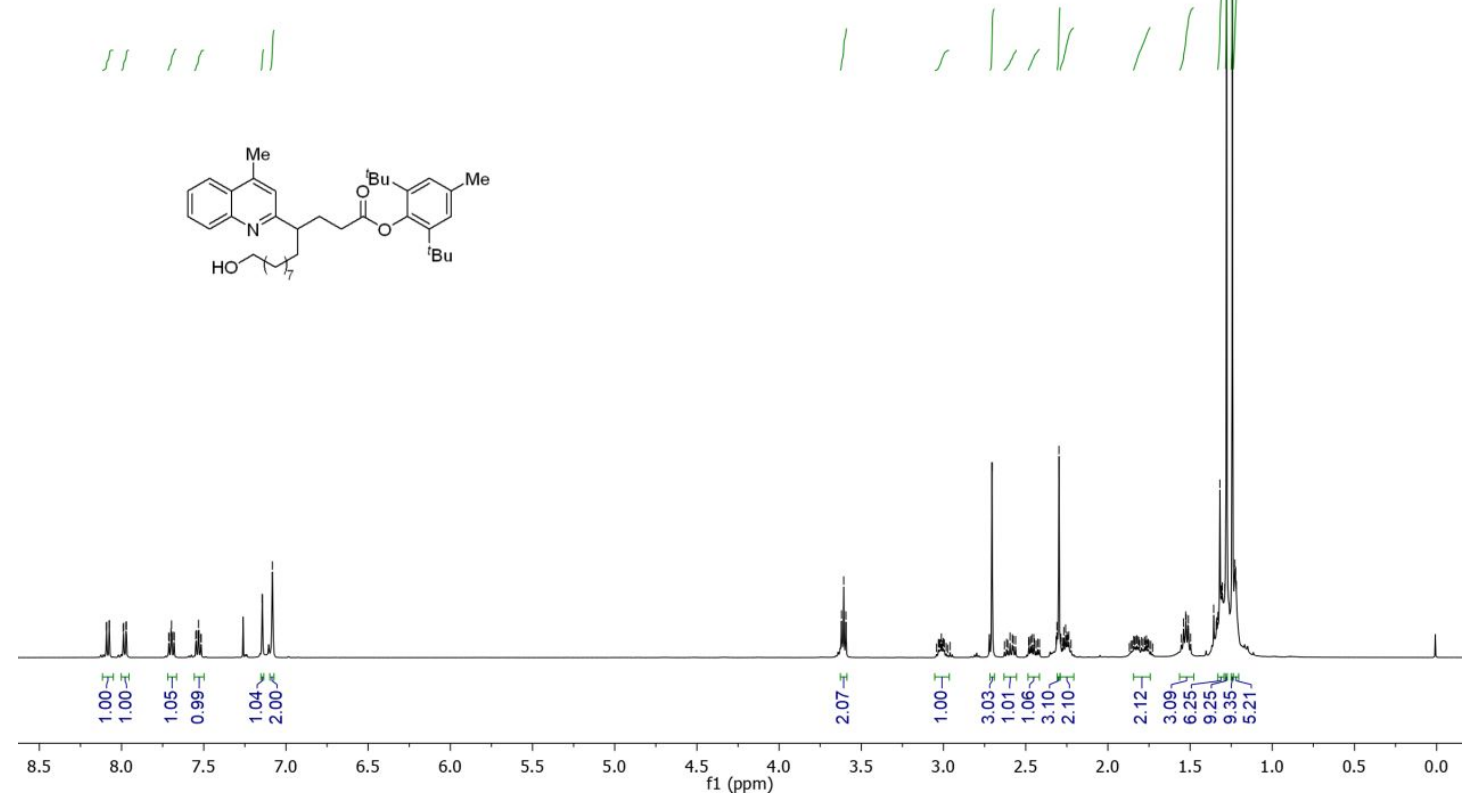

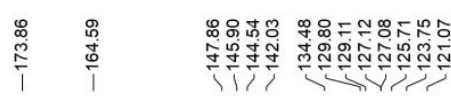

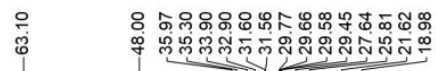
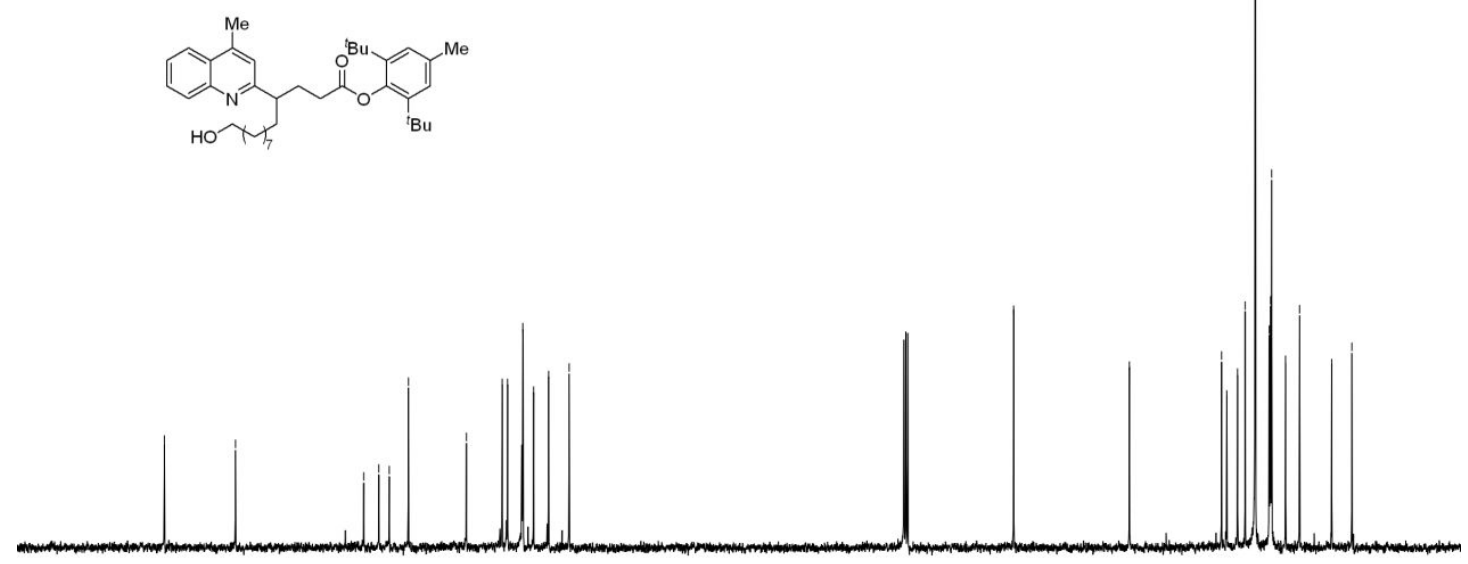

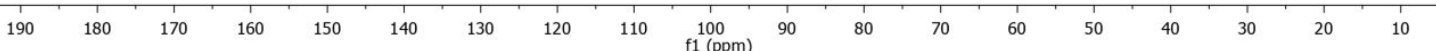


${ }^{1} \mathrm{H}$ NMR (400 MHz, $\left.\mathrm{CDCl}_{3}\right)$ and ${ }^{13} \mathrm{C}$ NMR (125 $\left.\mathrm{MHz}, \mathrm{CDCl}_{3}\right)$ spectra for $7 \mathrm{e}$

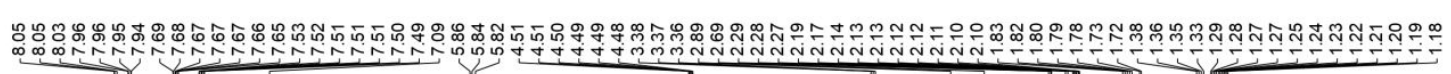
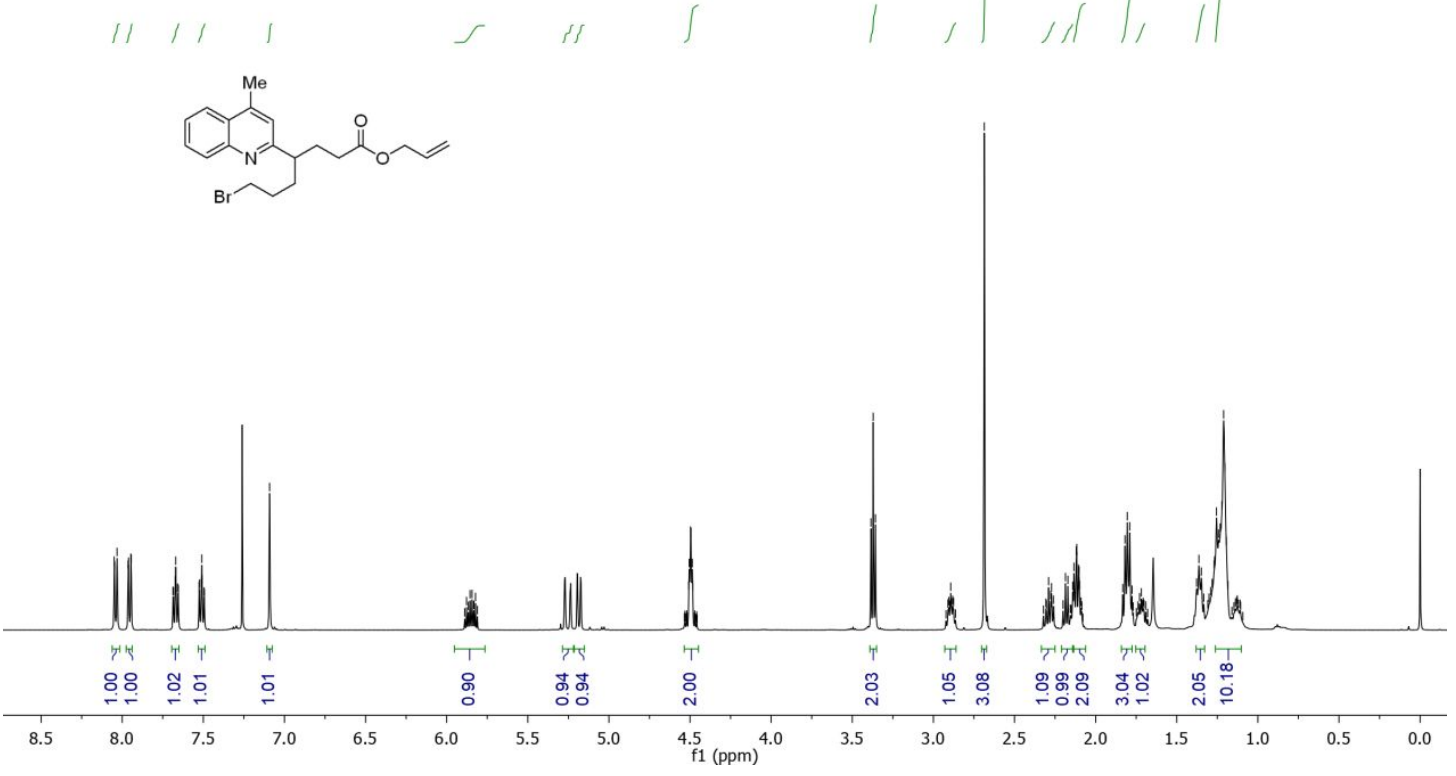

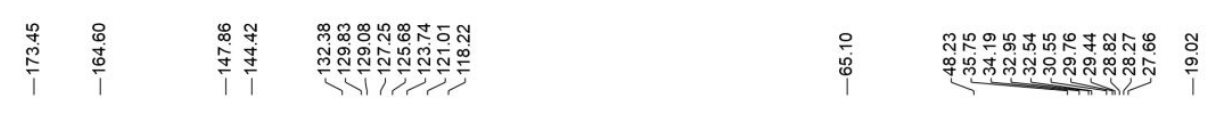

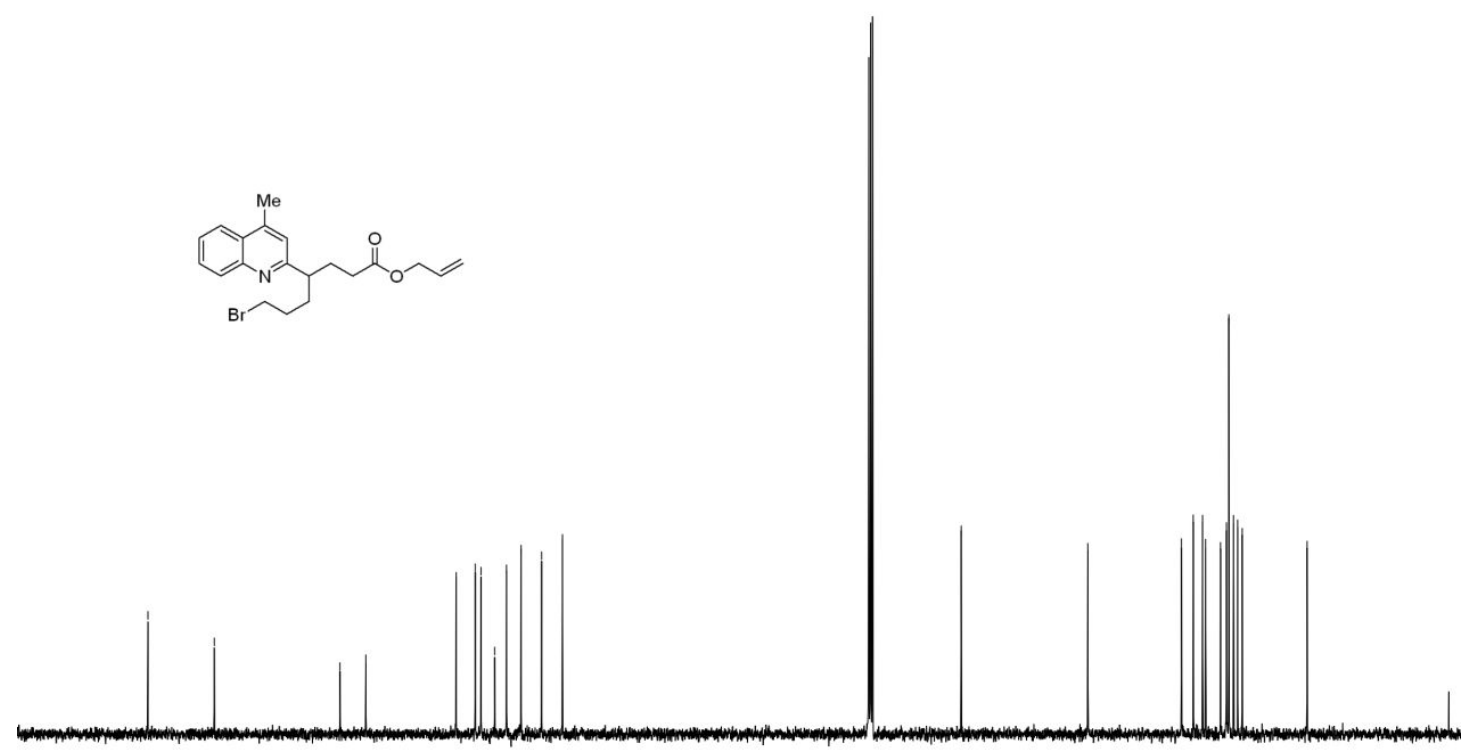

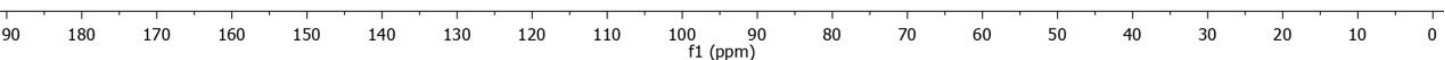


${ }^{1} \mathrm{H}$ NMR (400 MHz, $\left.\mathrm{CDCl}_{3}\right)$ and ${ }^{13} \mathrm{C}$ NMR (125 MHz, $\left.\mathrm{CDCl}_{3}\right)$ spectra for $7 \mathrm{f}$

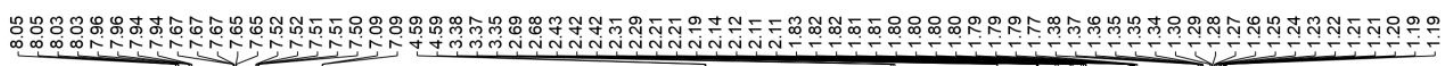
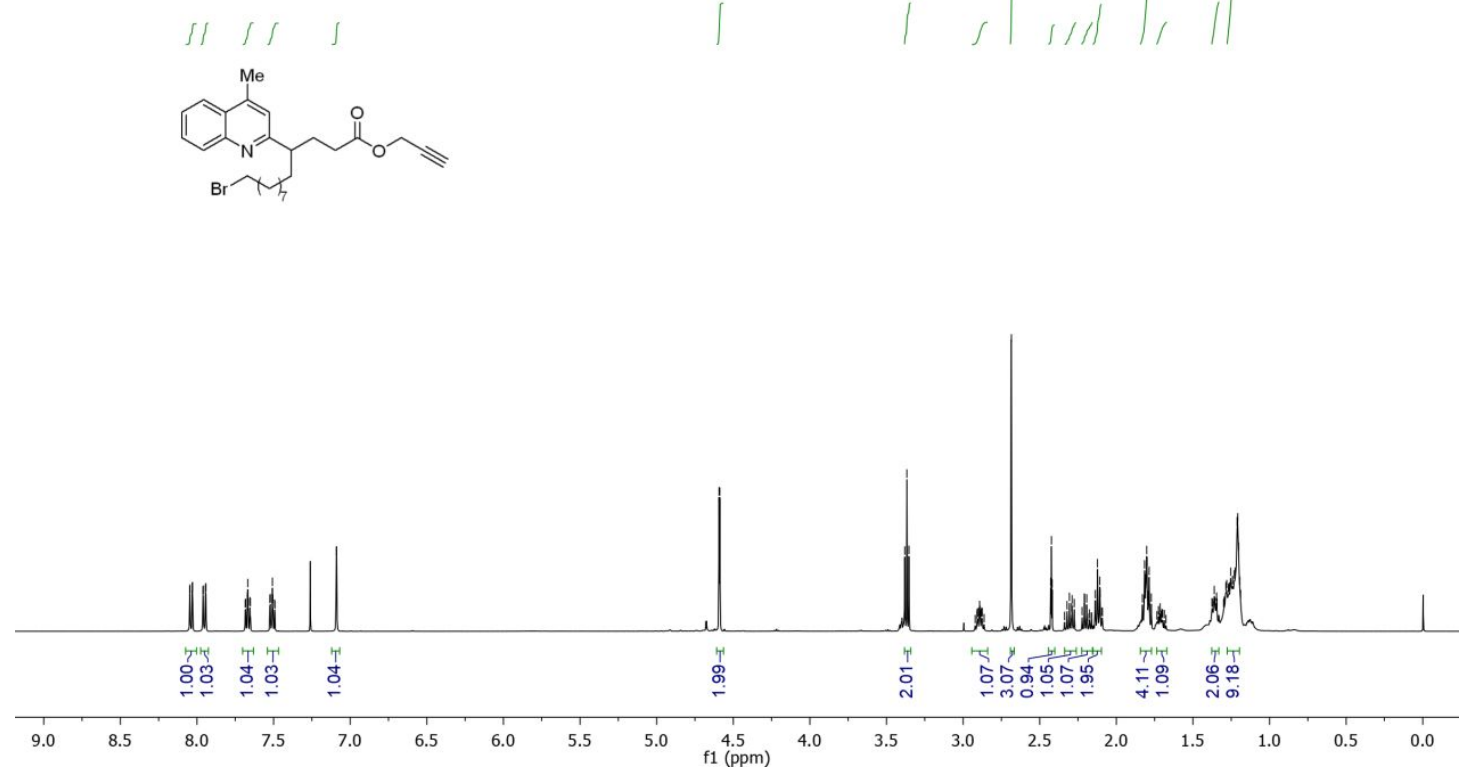

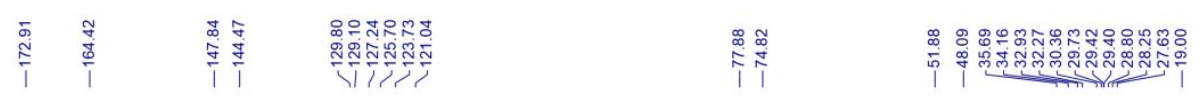

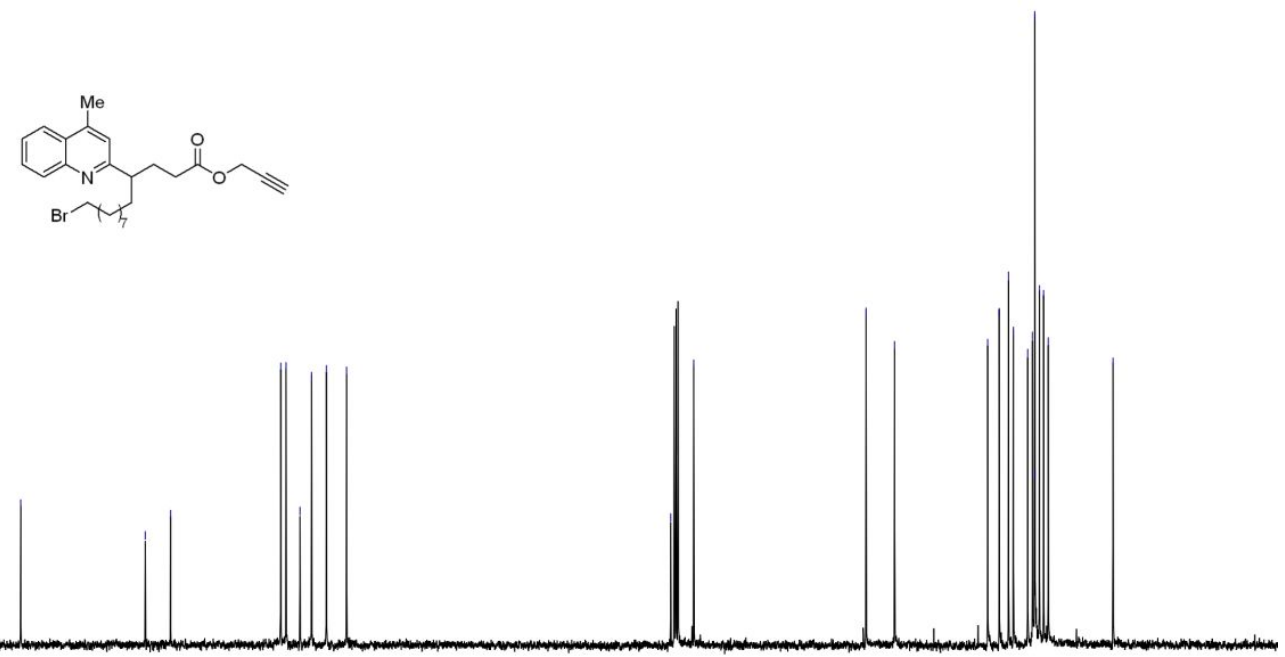

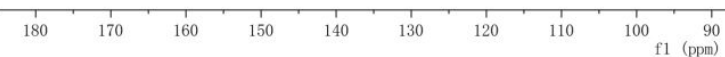


${ }^{1} \mathrm{H}$ NMR (400 MHz, $\left.\mathrm{CDCl}_{3}\right)$ and ${ }^{13} \mathrm{C}$ NMR (125 $\left.\mathrm{MHz}, \mathrm{CDCl}_{3}\right)$ spectra for $7 \mathrm{~g}$

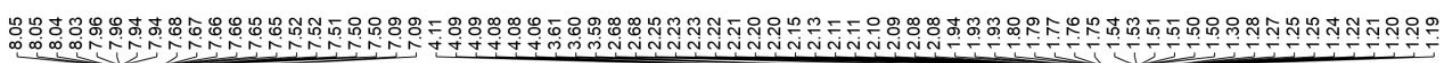

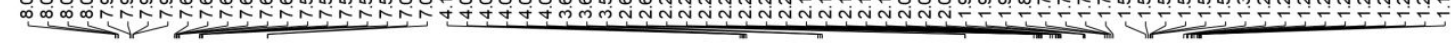
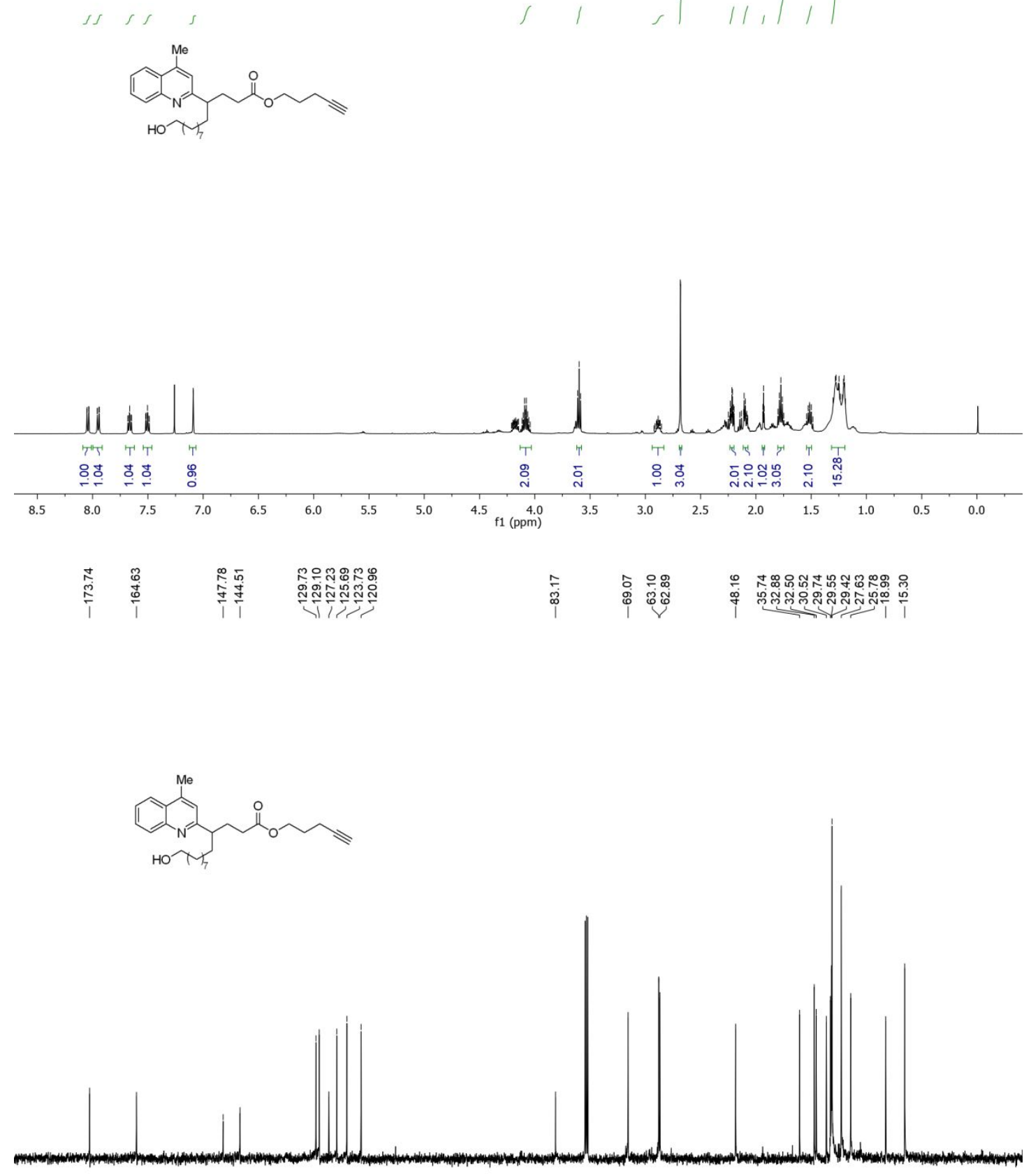

180

$170 \quad 160$

150

$140 \quad 130$

$120 \quad 110 \quad 100$ f1 $\stackrel{90}{(\mathrm{ppm})}$

80

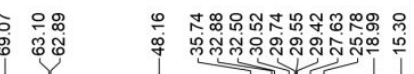


${ }^{1} \mathrm{H}$ NMR (400 MHz, $\left.\mathrm{CDCl}_{3}\right)$ and ${ }^{13} \mathrm{C}$ NMR (125 $\left.\mathrm{MHz}, \mathrm{CDCl}_{3}\right)$ spectra for $7 \mathrm{~h}$

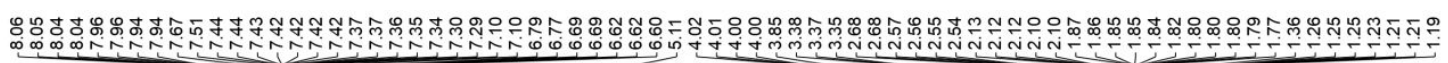
SI S Jllis is<smiles>CCCCCC(=O)OCCCc1cc(C)cc(C)c1</smiles>

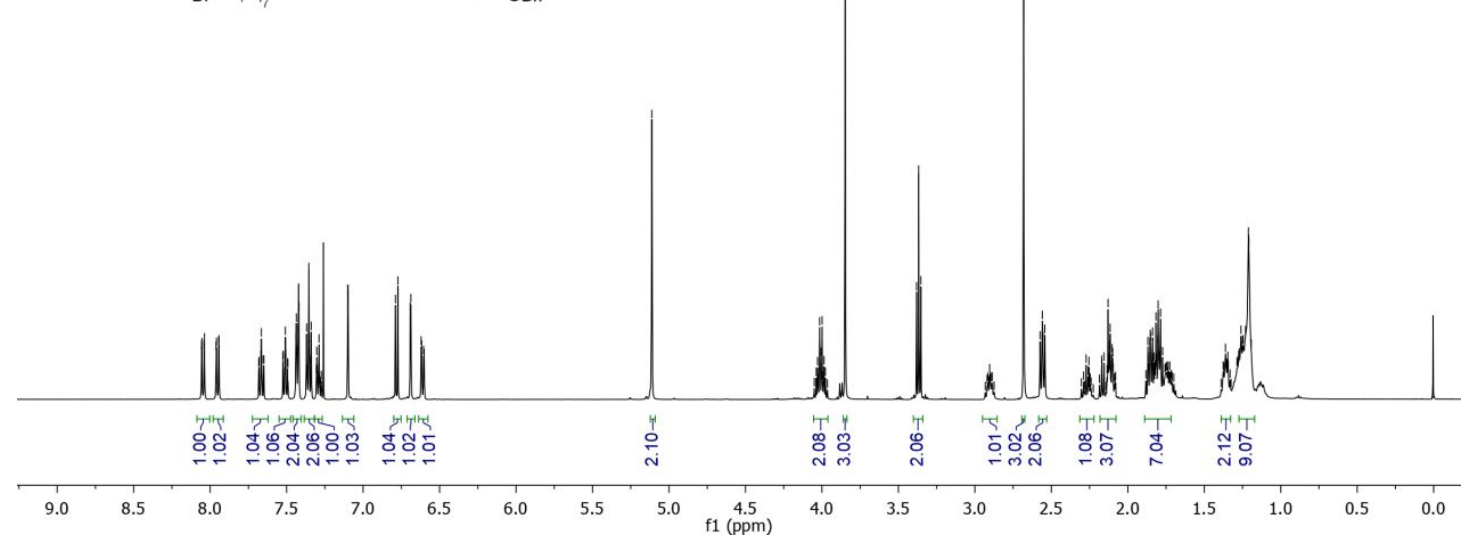

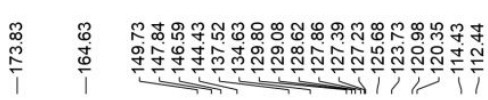
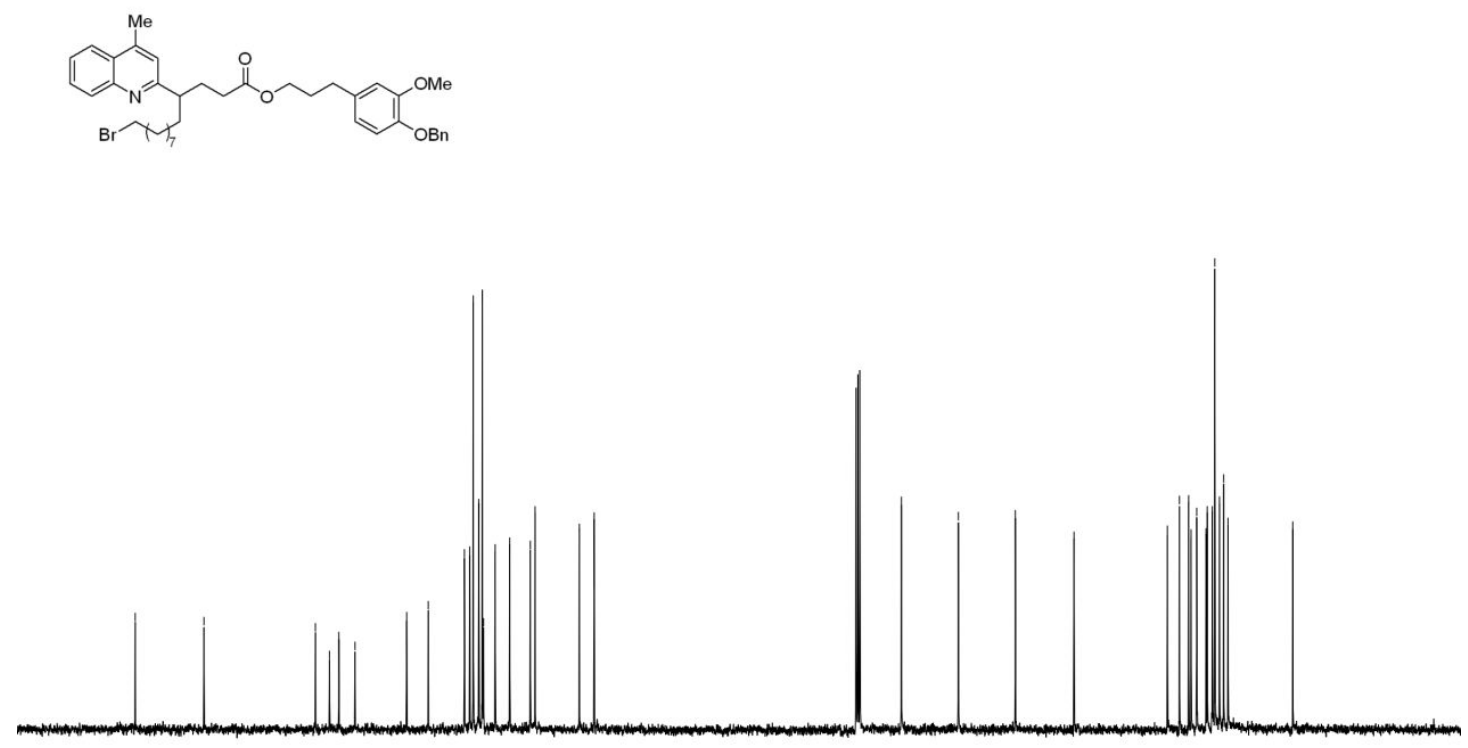

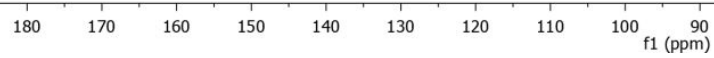


${ }^{1} \mathrm{H}$ NMR (400 MHz, $\left.\mathrm{CDCl}_{3}\right)$ and ${ }^{13} \mathrm{C}$ NMR (125 MHz, $\left.\mathrm{CDCl}_{3}\right)$ spectra for $7 \mathrm{i}$

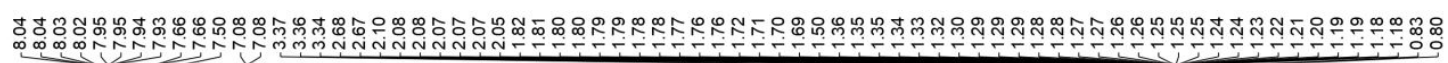
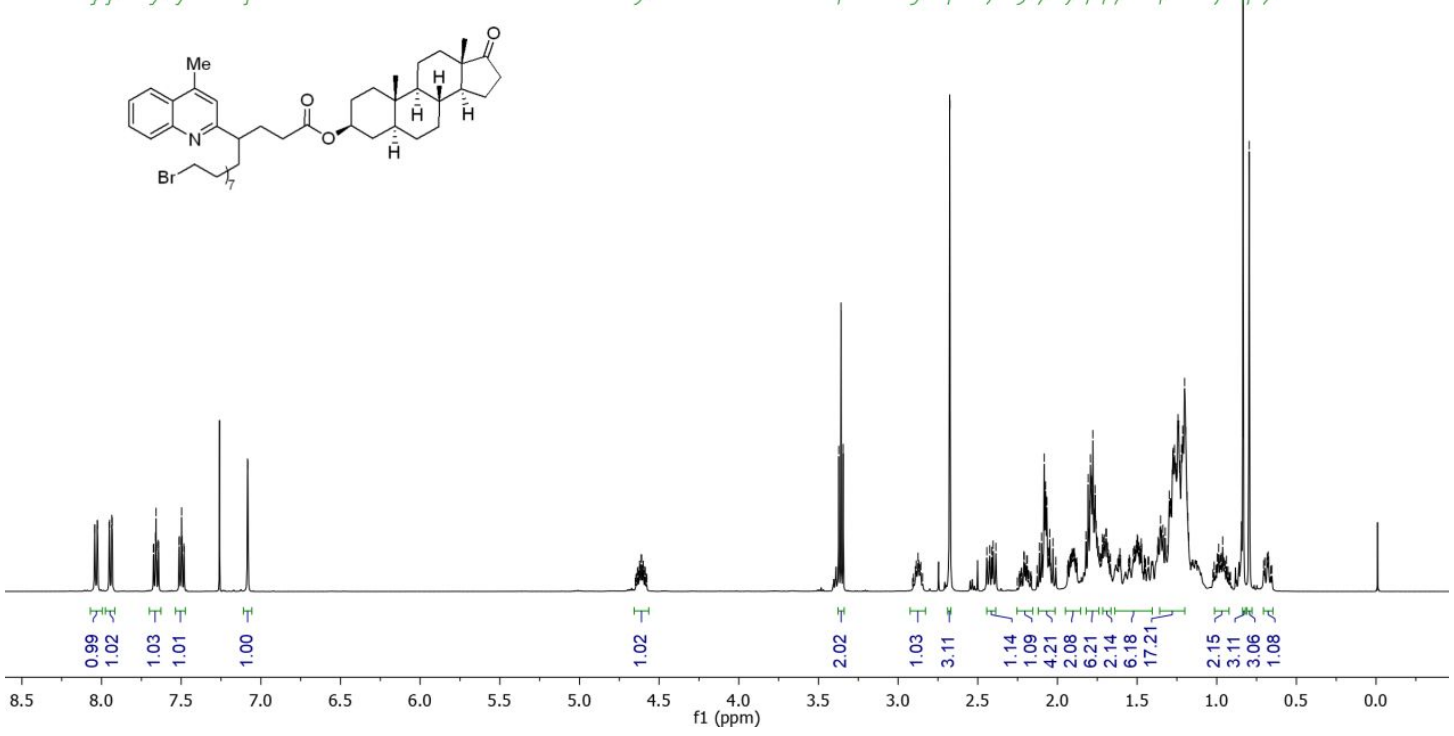
إن
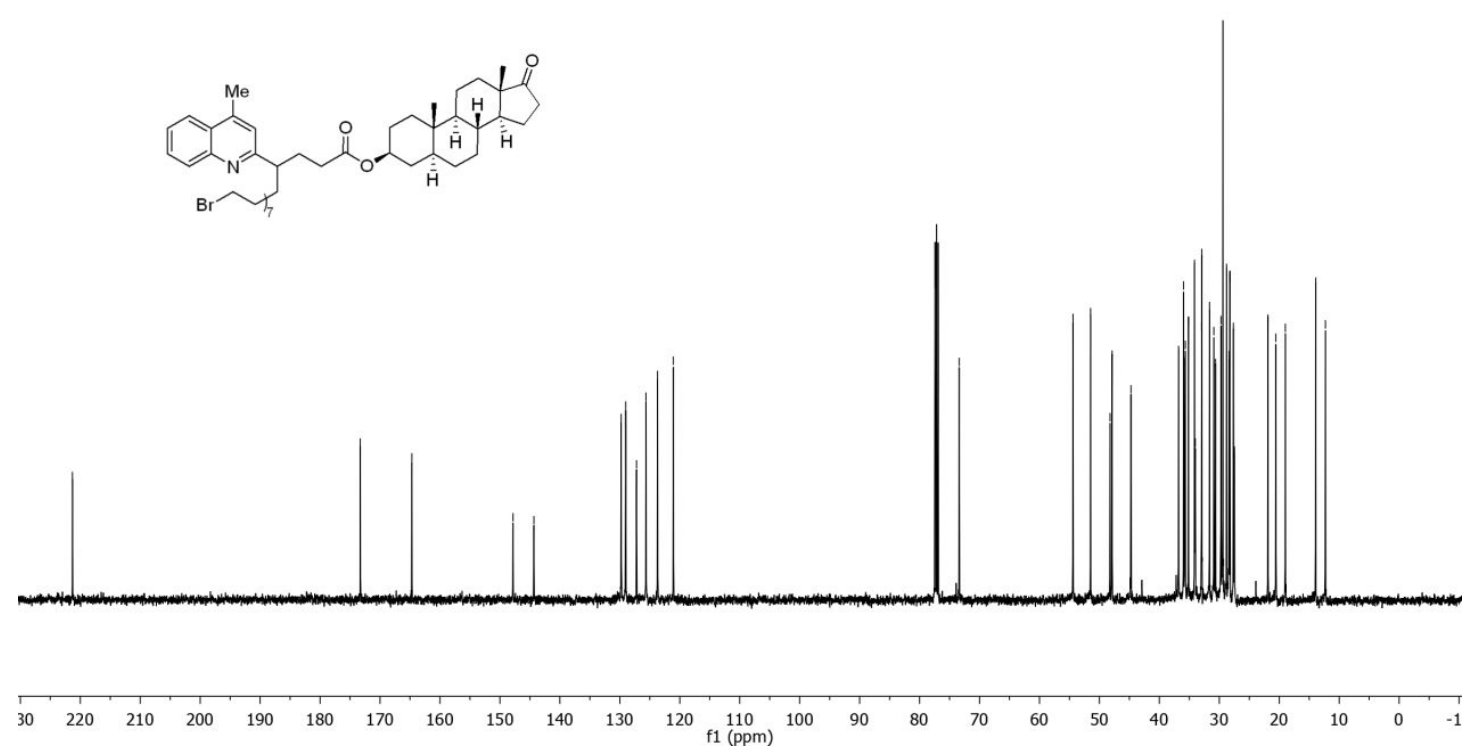
${ }^{1} \mathrm{H}$ NMR (400 MHz, $\left.\mathrm{CDCl}_{3}\right)$ and ${ }^{13} \mathrm{C}$ NMR (125 $\left.\mathrm{MHz}, \mathrm{CDCl}_{3}\right)$ spectra for $7 \mathbf{j}$

$\underbrace{800}$
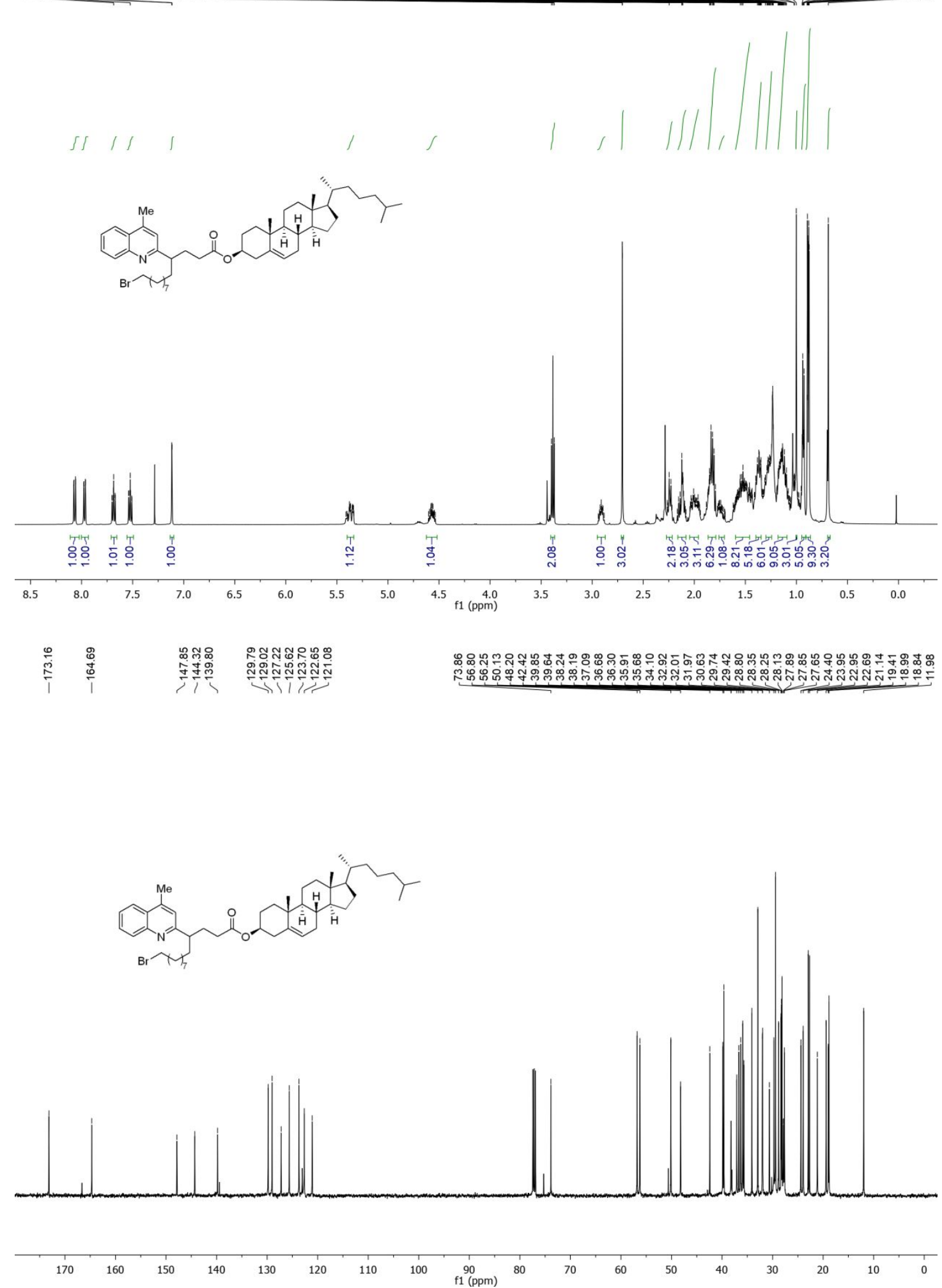
${ }^{1} \mathrm{H}$ NMR (400 $\left.\mathrm{MHz}, \mathrm{CDCl}_{3}\right)$ and ${ }^{13} \mathrm{C}$ NMR (125 $\left.\mathrm{MHz}, \mathrm{CDCl}_{3}\right)$ spectra for $7 \mathrm{k}$

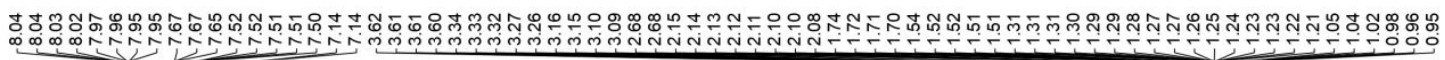

SS S S S

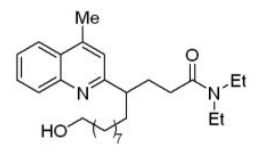

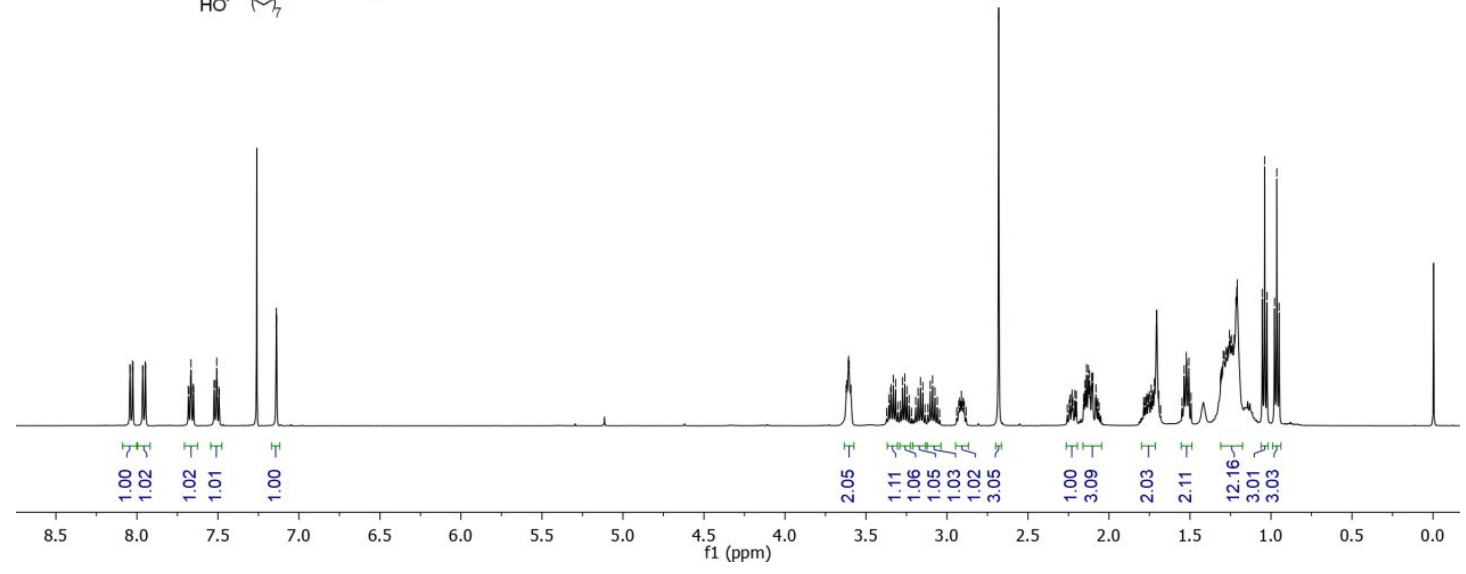

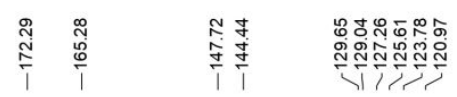

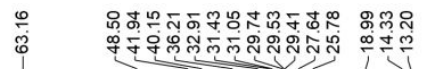
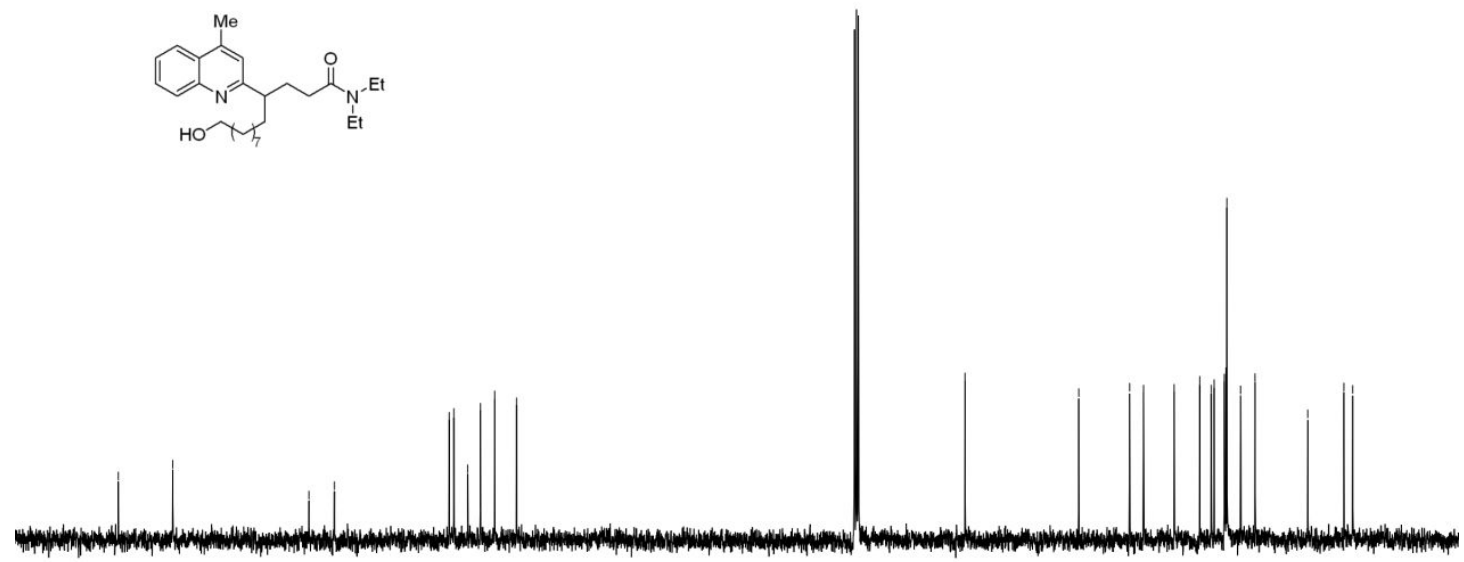

170

160

150

(1900)

12

100

f1 (ppm)

$\begin{array}{lllllllll}1 & 1 & 1 & 1 & 1 & 1 & 1 & 10 & 0\end{array}$ 
${ }^{1} \mathrm{H}$ NMR (400 MHz, $\left.\mathrm{CDCl}_{3}\right)$ and ${ }^{13} \mathrm{C}$ NMR (125 $\left.\mathrm{MHz}, \mathrm{CDCl}_{3}\right)$ spectra for $7 \mathrm{l}$

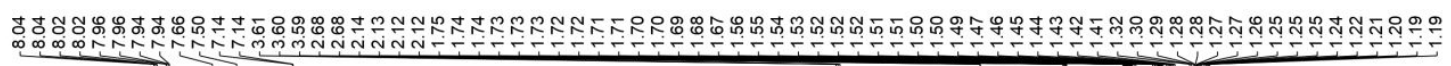

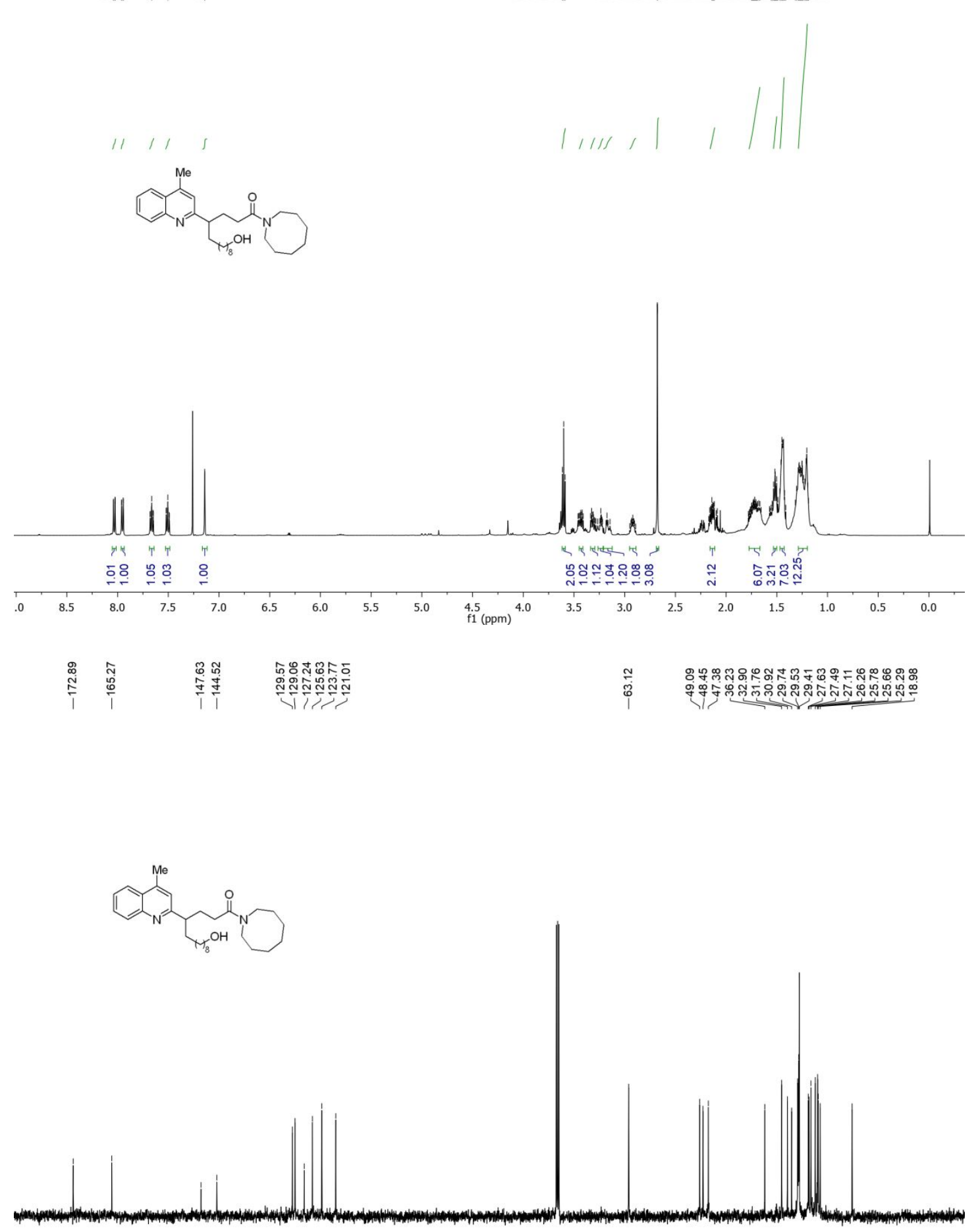

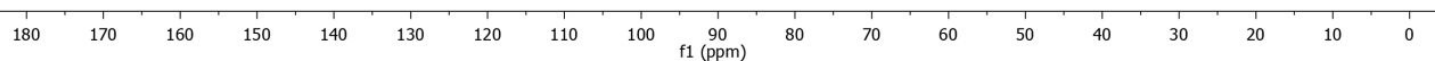


${ }^{1} \mathrm{H}$ NMR (400 MHz, $\left.\mathrm{CDCl}_{3}\right)$ and ${ }^{13} \mathrm{C}$ NMR (125 $\left.\mathrm{MHz}, \mathrm{CDCl}_{3}\right)$ spectra for $7 \mathrm{~m}$

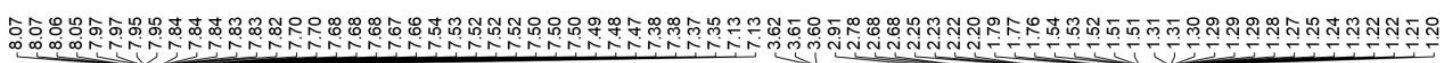
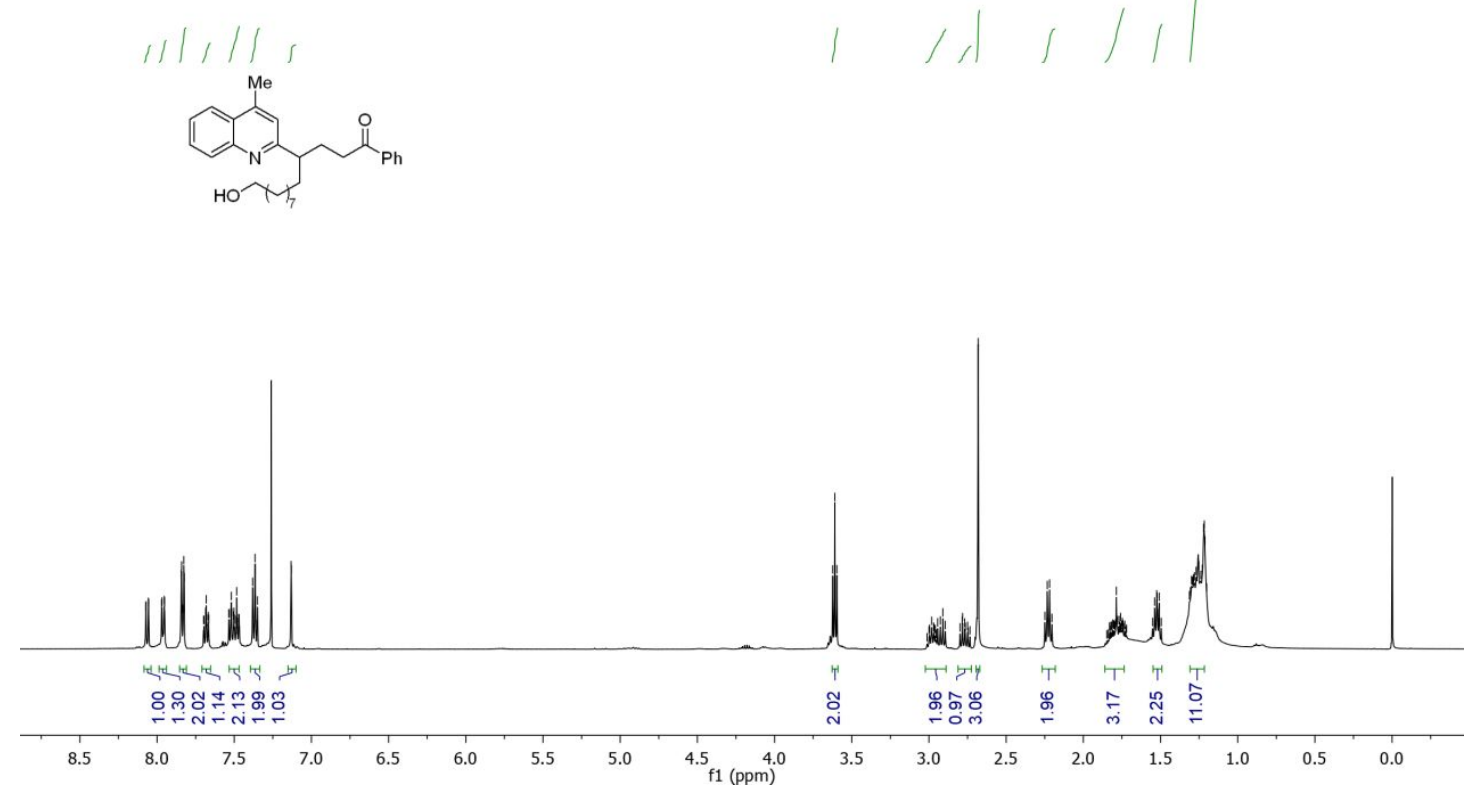

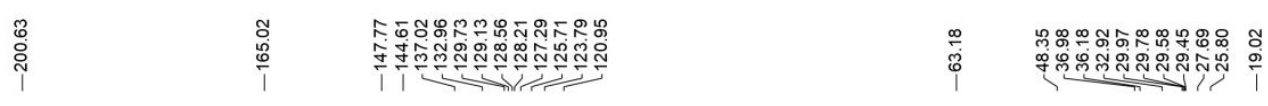
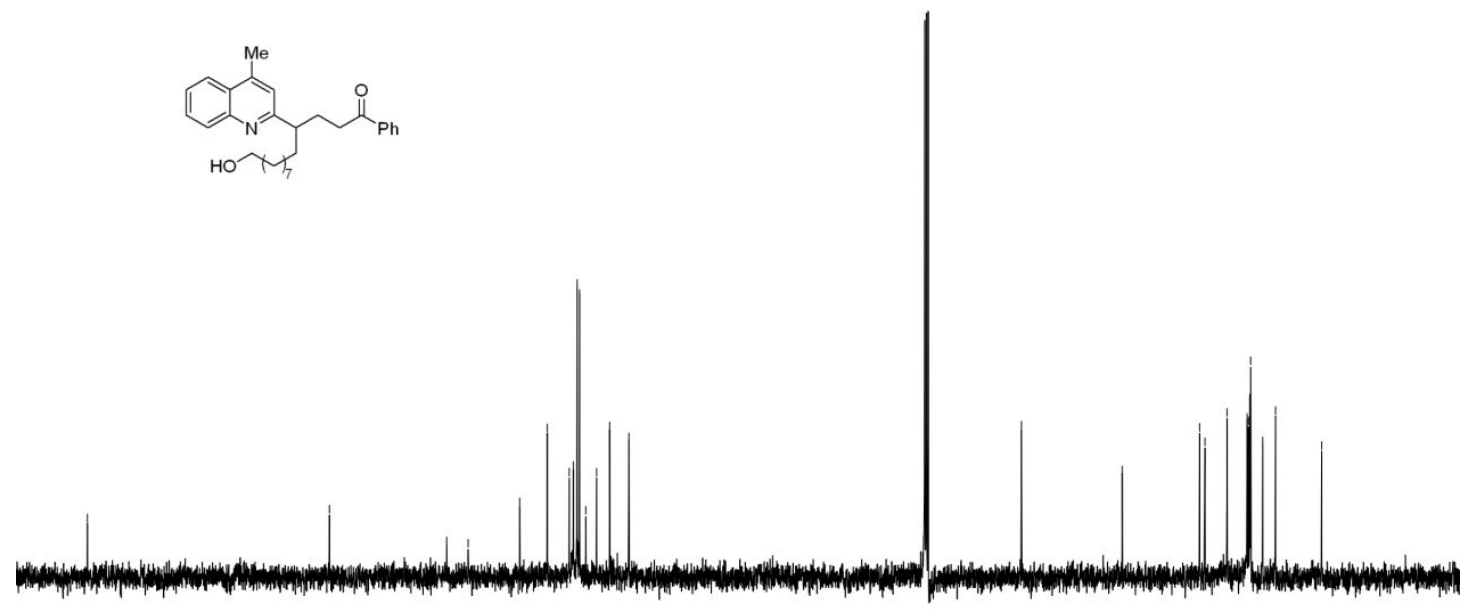

$\begin{array}{lllllllllllllllllllllllll}1 & 1 \\ 10 & 200 & 190 & 180 & 170 & 160 & 150 & 140 & 130 & 120 & \underset{\mathrm{f} 1}{1}(\mathrm{ppm}) & 100 & 90 & 80 & 70 & 60 & 50 & 40 & 30 & 20 & 10 & 0\end{array}$ 
${ }^{1} \mathrm{H}$ NMR (400 MHz, $\mathrm{CDCl}_{3}$ ) and ${ }^{13} \mathrm{C}$ NMR (125 $\left.\mathrm{MHz}, \mathrm{CDCl}_{3}\right)$ spectra for $7 \mathrm{n}$

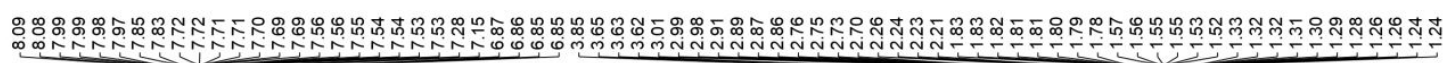
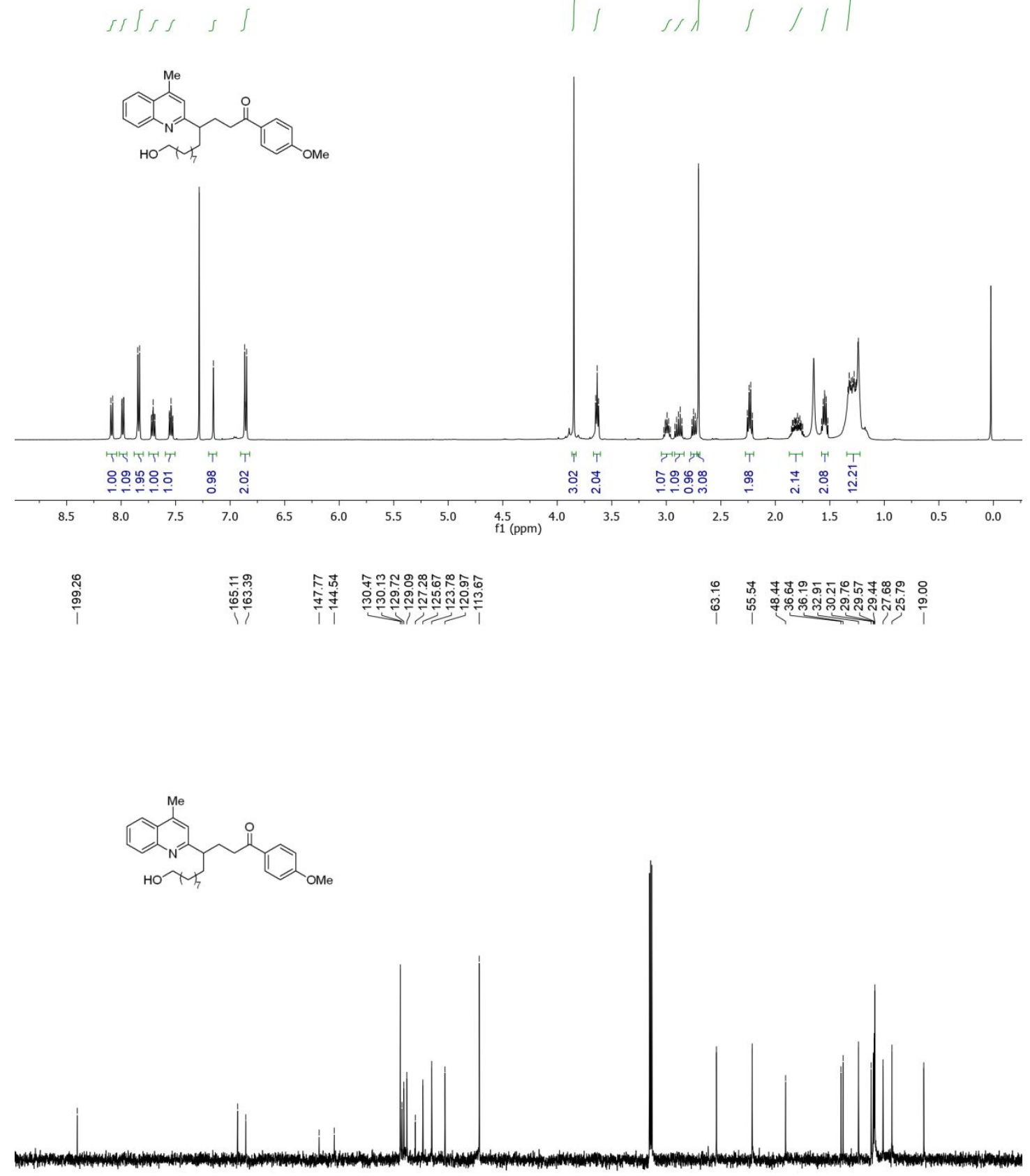

$\begin{array}{lllllllllll}210 & 200 & 190 & 180 & 170 & 160 & 150 & 140 & 130 & 120 & 110 \\ \mathrm{f} 1(\mathrm{ppm}) & 100\end{array}$ 
${ }^{1} \mathrm{H}$ NMR (400 MHz, $\left.\mathrm{CDCl}_{3}\right)$ and ${ }^{13} \mathrm{C}$ NMR (125 MHz, $\left.\mathrm{CDCl}_{3}\right)$ spectra for 70

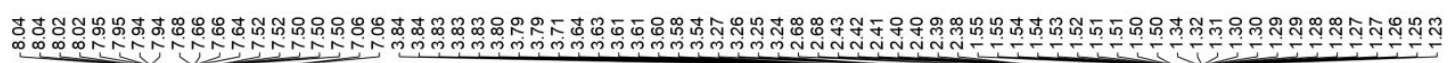

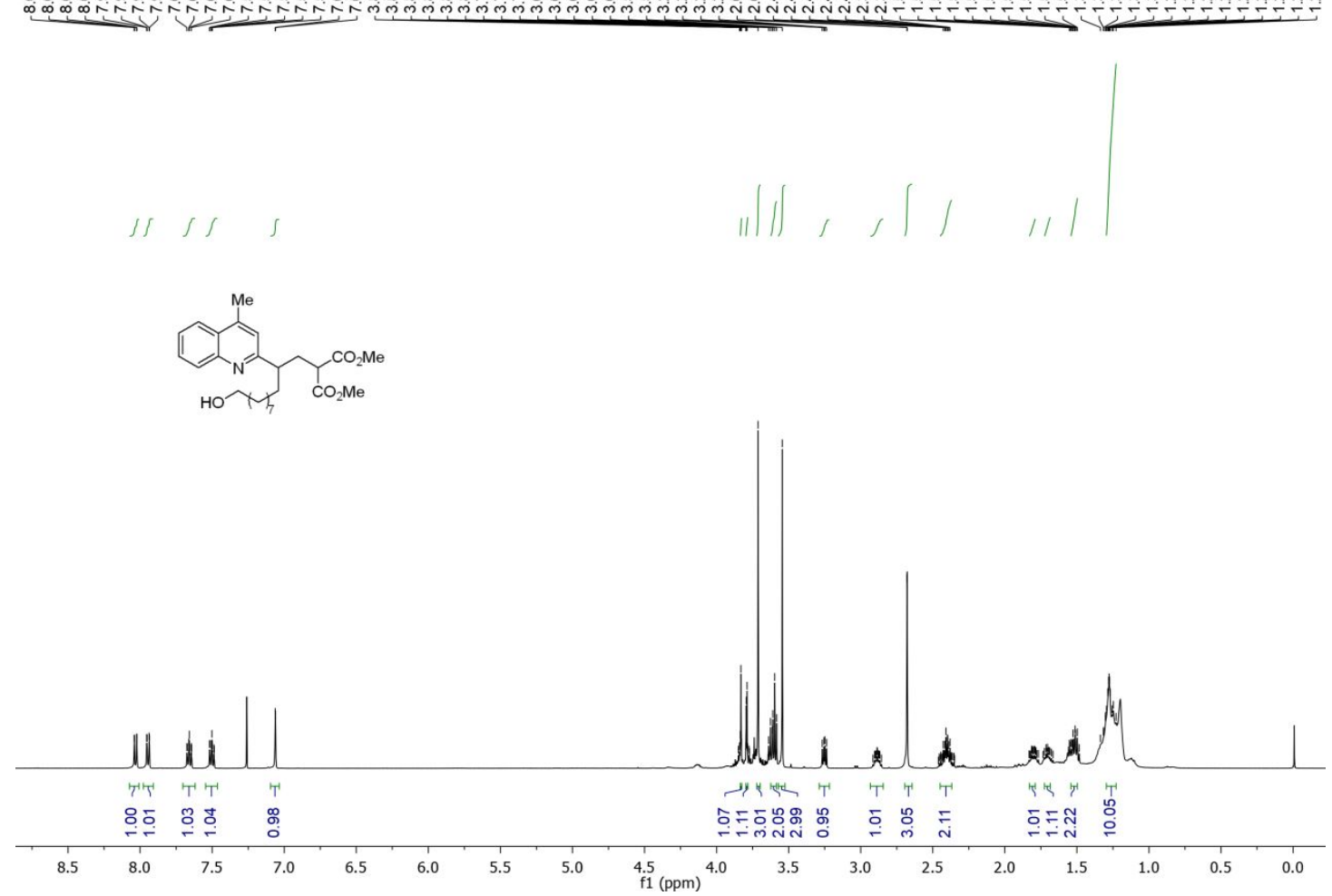

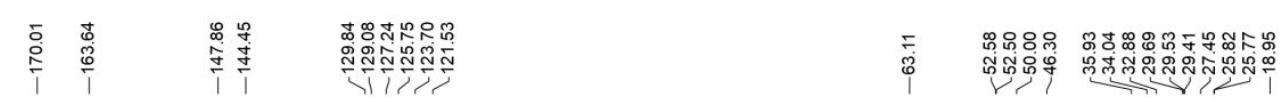
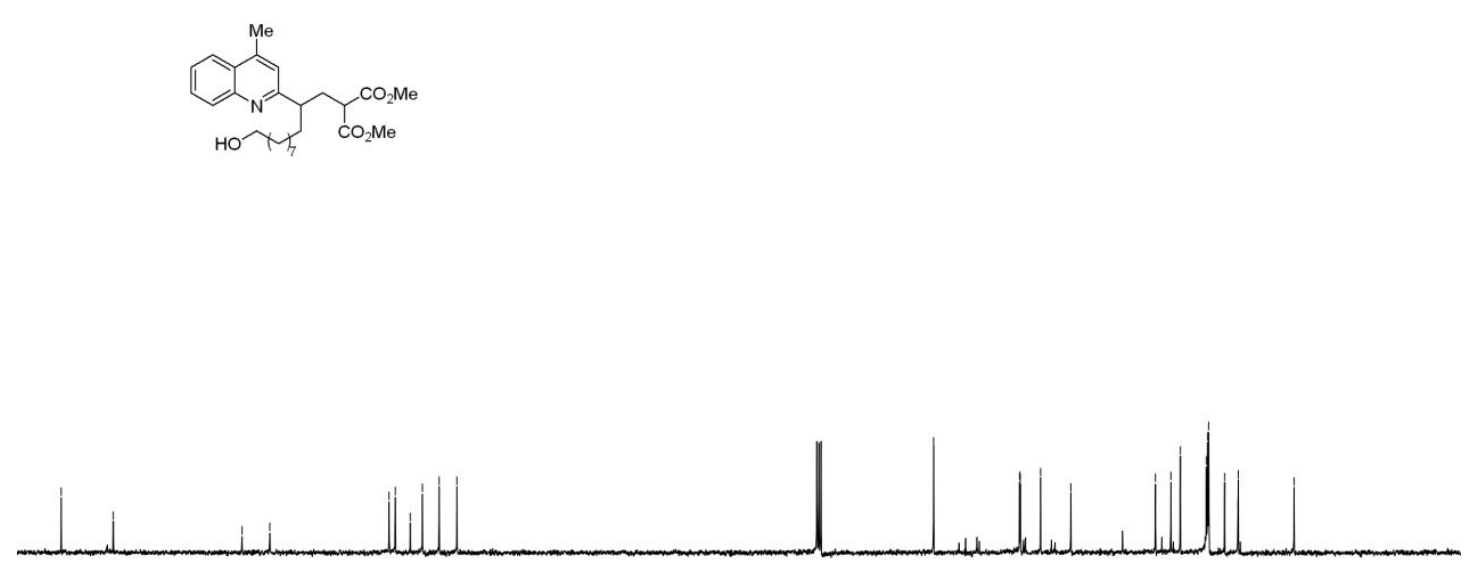

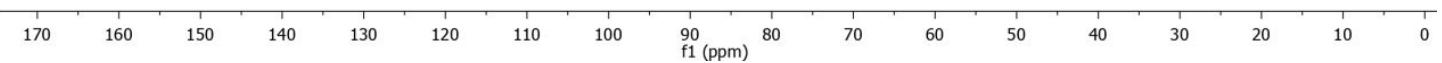


${ }^{1} \mathrm{H}$ NMR (400 MHz, $\left.\mathrm{CDCl}_{3}\right)$ and ${ }^{13} \mathrm{C}$ NMR (125 MHz, $\left.\mathrm{CDCl}_{3}\right)$ spectra for $7 \mathrm{p}$

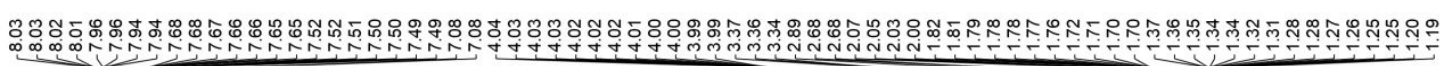
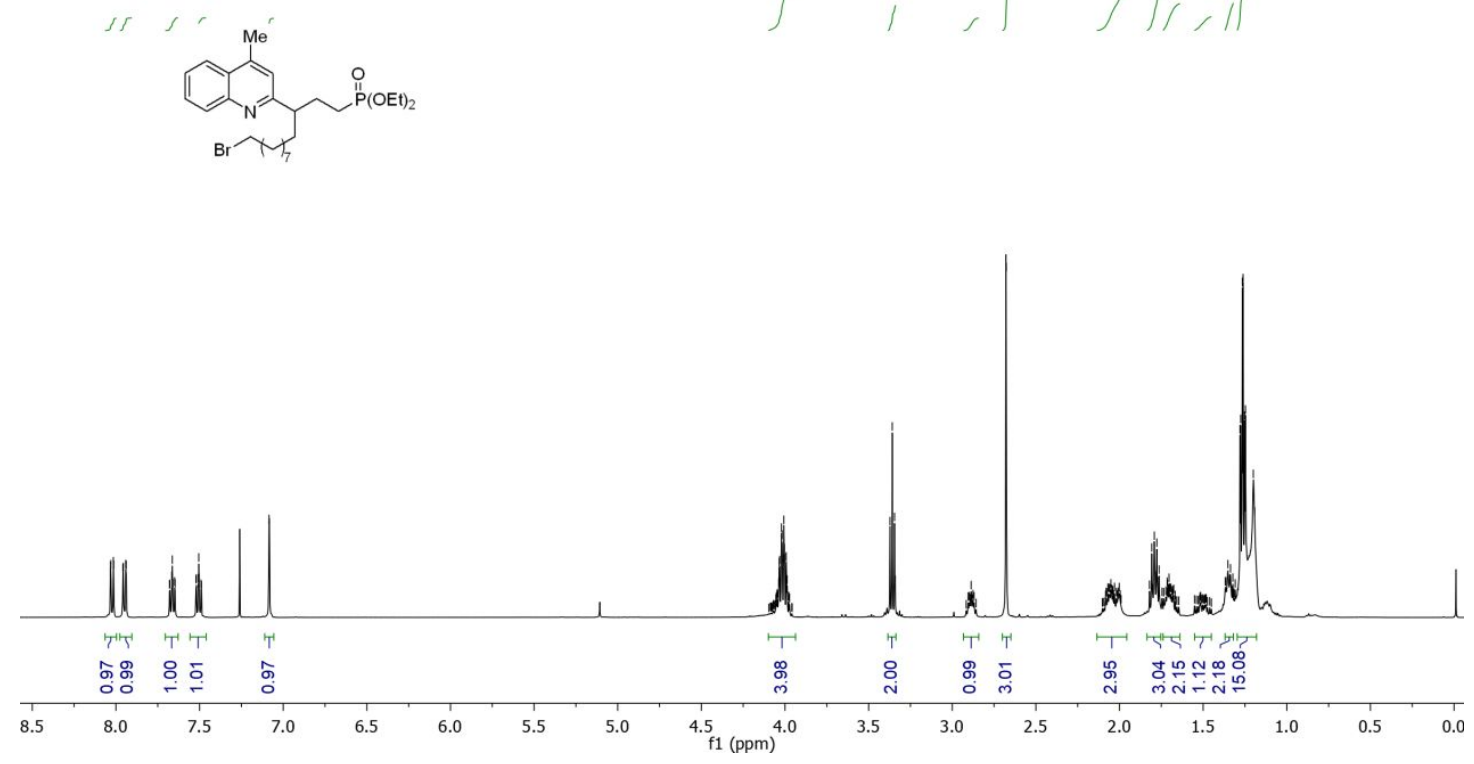

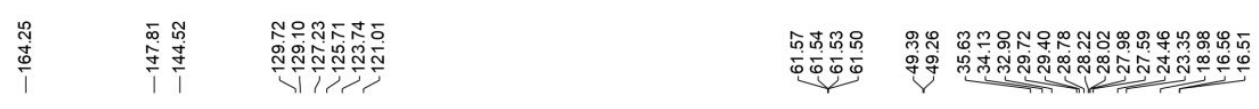
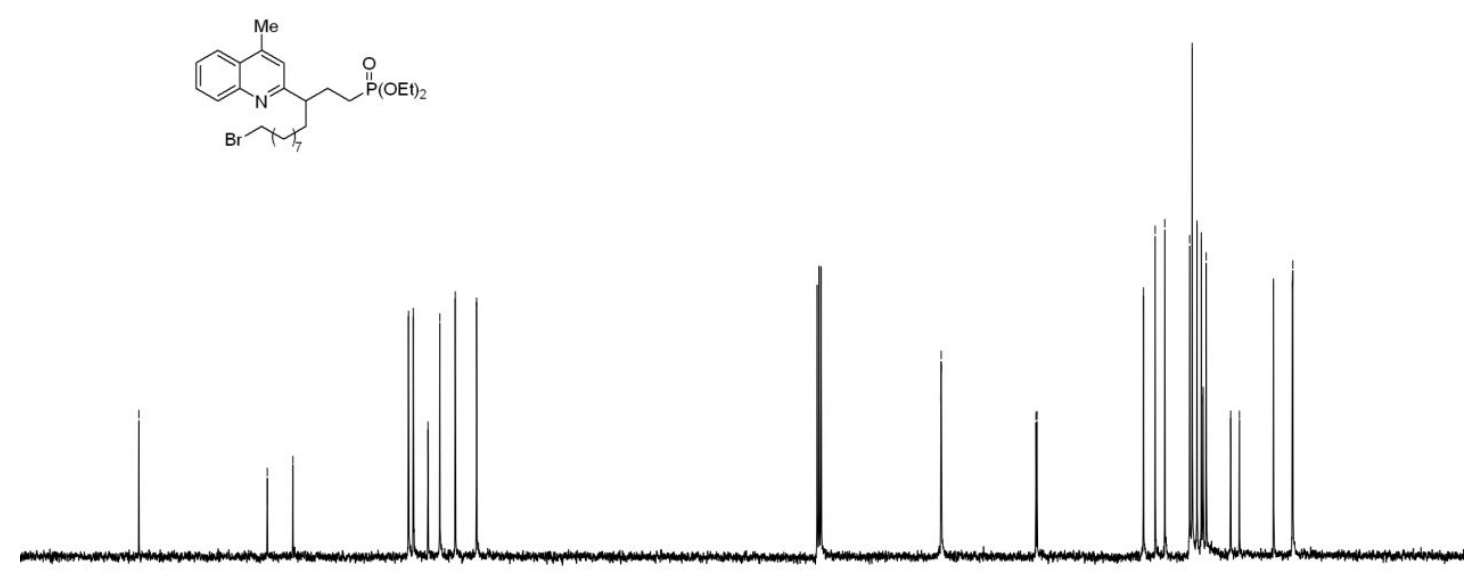

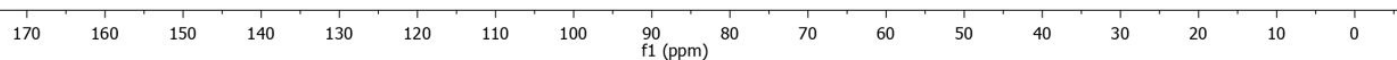


${ }^{1} \mathrm{H}$ NMR (400 MHz, $\left.\mathrm{CDCl}_{3}\right)$ and ${ }^{13} \mathrm{C}$ NMR (125 $\left.\mathrm{MHz}, \mathrm{CDCl}_{3}\right)$ spectra for $7 \mathrm{q}$

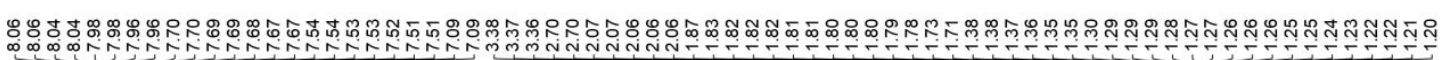

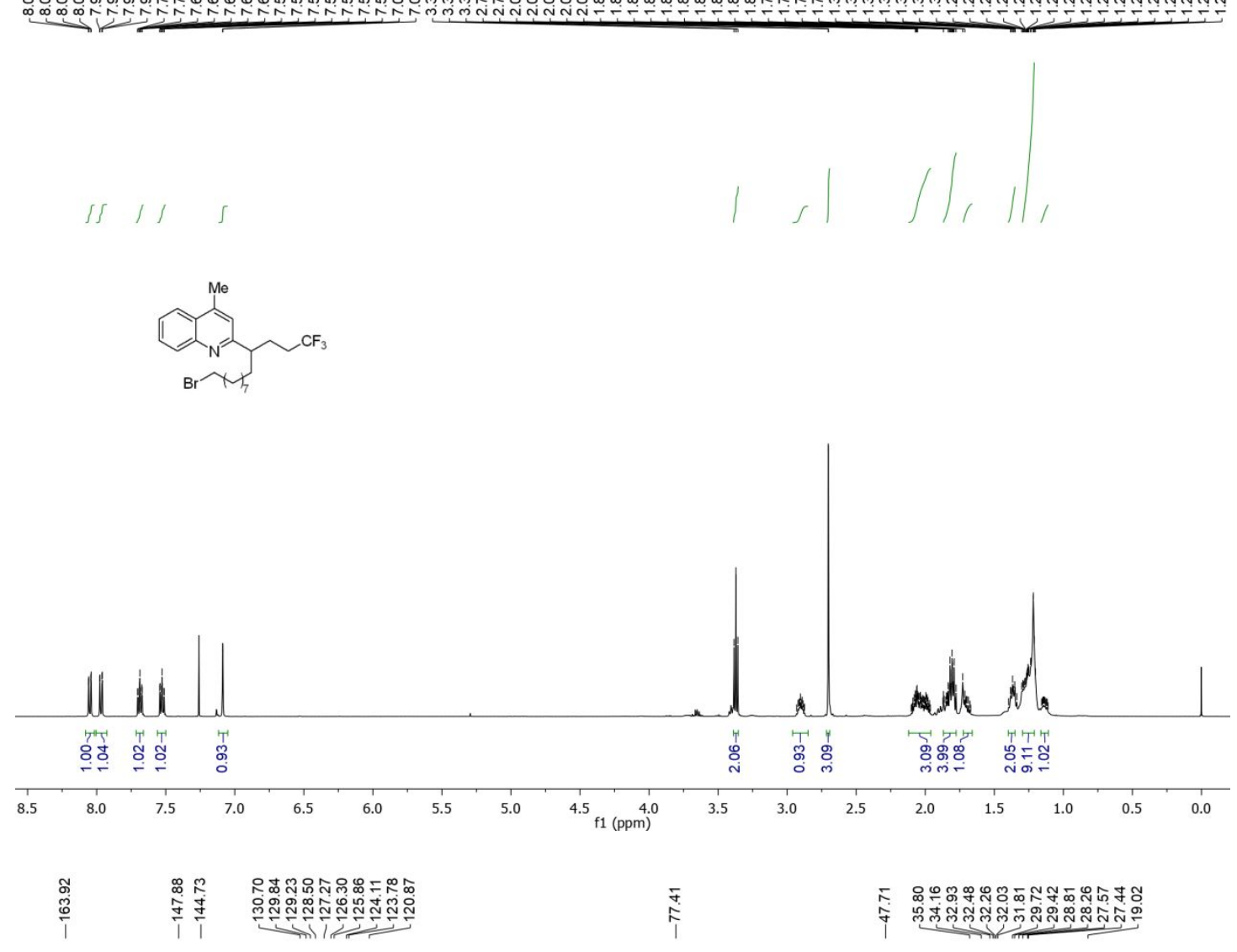<smiles>Cc1cc(C(CCC(F)(F)F)CCC(F)(F)F)nc2ccccc12</smiles>

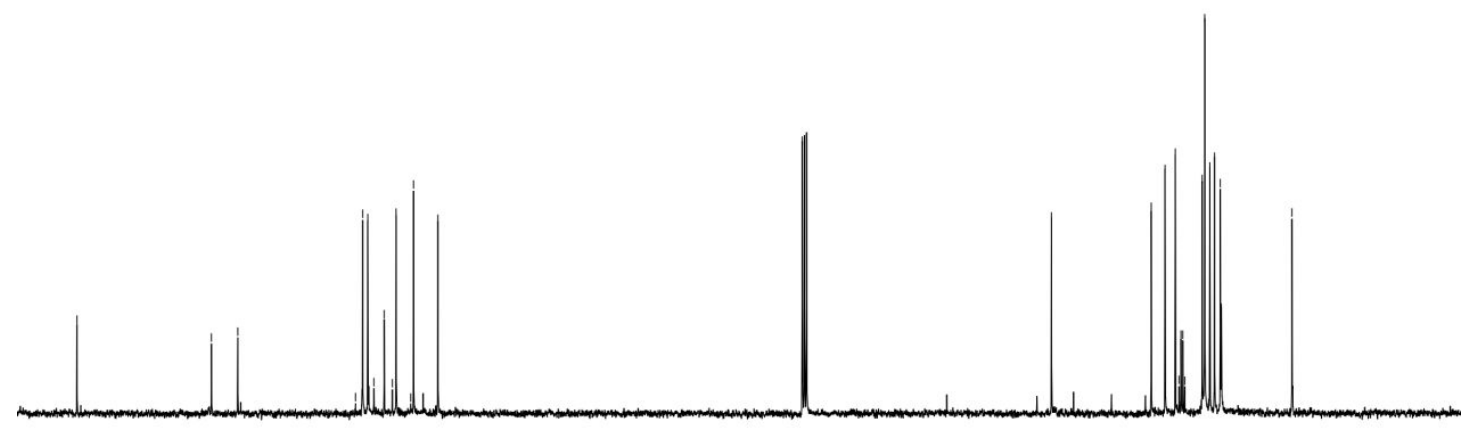

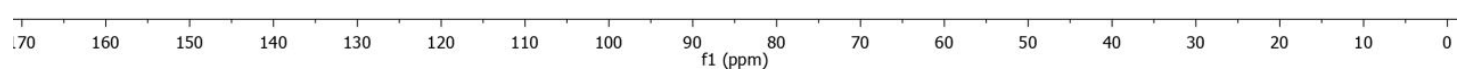


${ }^{1} \mathrm{H}$ NMR (400 MHz, $\left.\mathrm{CDCl}_{3}\right)$ and ${ }^{13} \mathrm{C}$ NMR (125 MHz, $\left.\mathrm{CDCl}_{3}\right)$ spectra for 8a

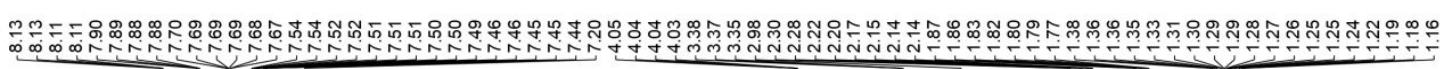
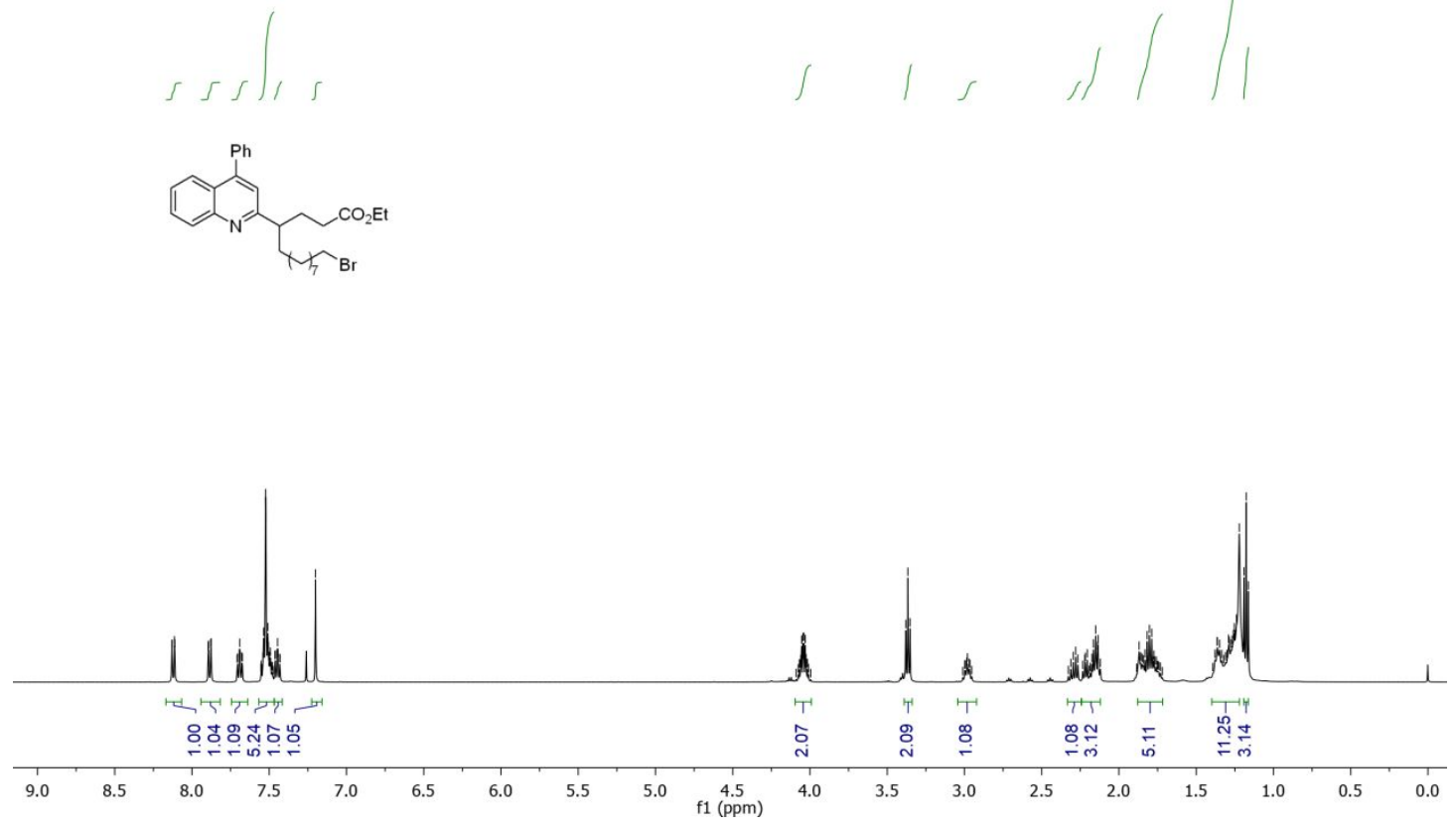

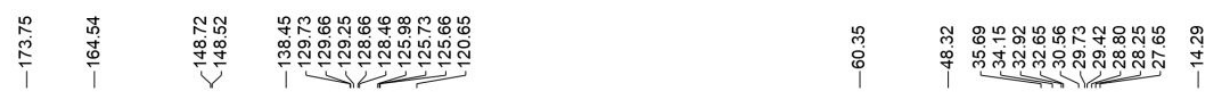

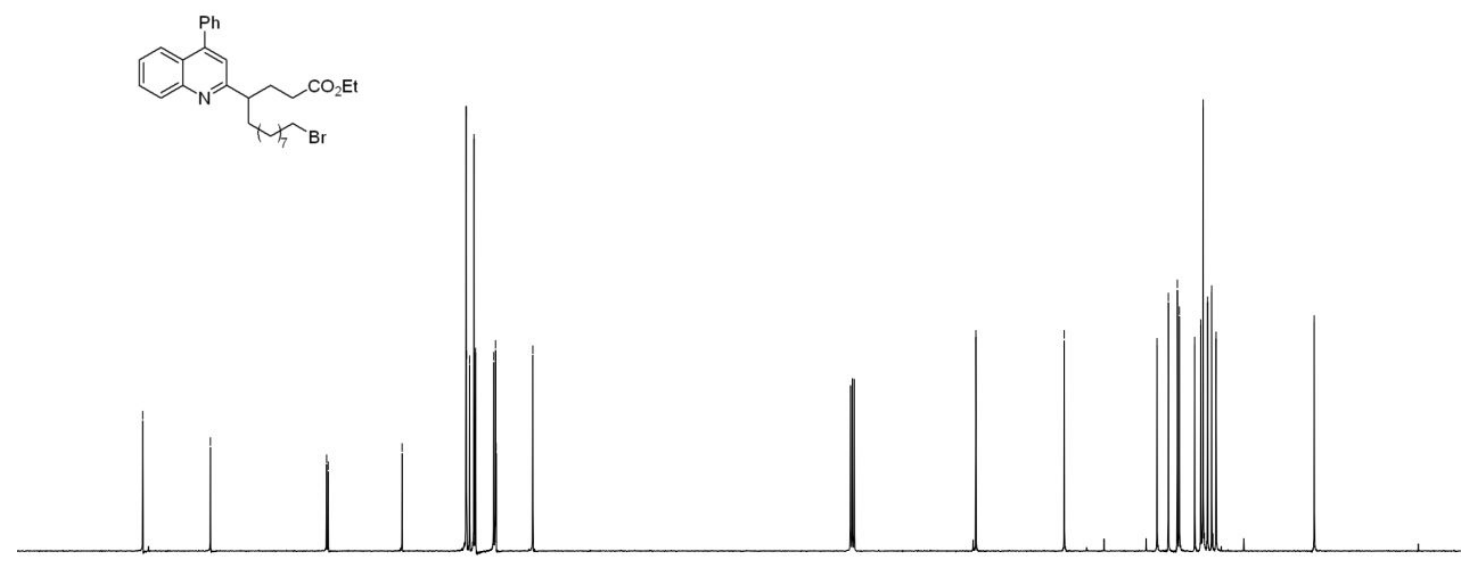

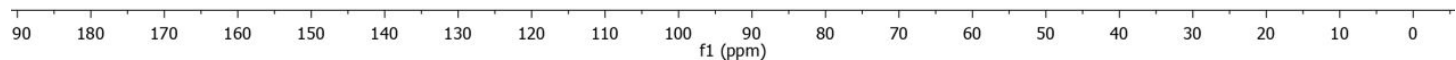


${ }^{1} \mathrm{H}$ NMR (400 MHz, $\left.\mathrm{CDCl}_{3}\right)$ and ${ }^{13} \mathrm{C}$ NMR (125 MHz, $\left.\mathrm{CDCl}_{3}\right)$ spectra for $8 \mathrm{~b}$

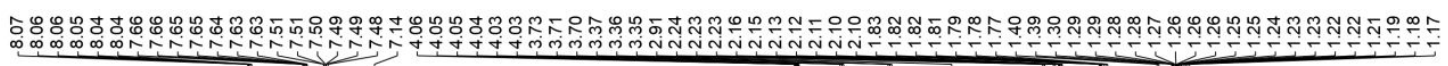

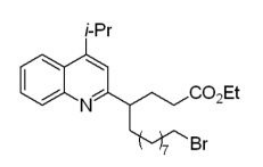

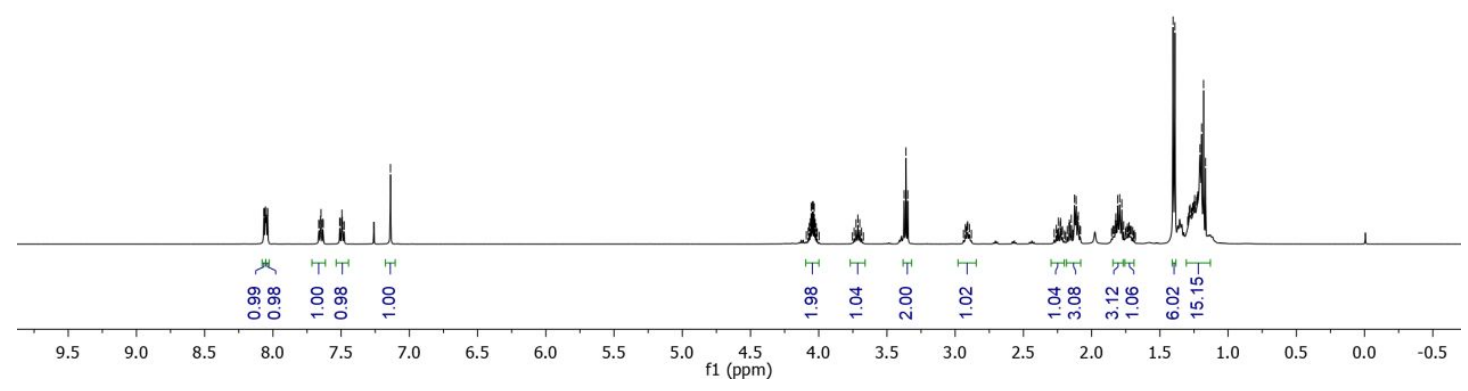

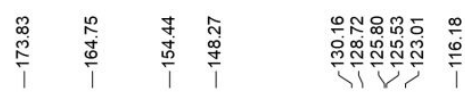

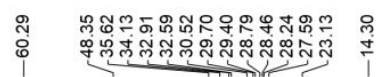

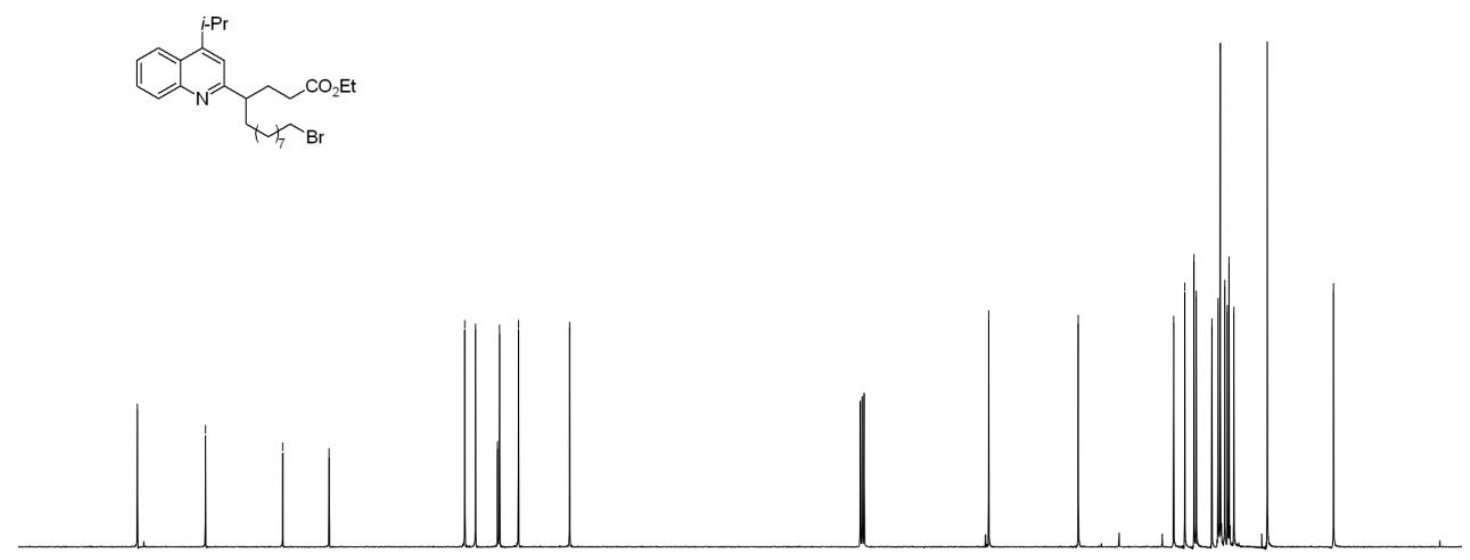

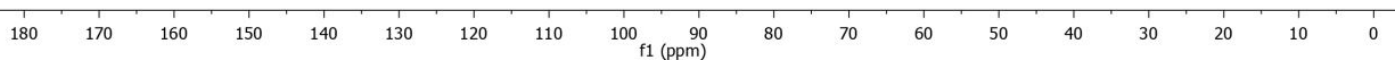


${ }^{1} \mathrm{H}$ NMR (400 MHz, $\left.\mathrm{CDCl}_{3}\right)$ and ${ }^{13} \mathrm{C}$ NMR (125 MHz, $\left.\mathrm{CDCl}_{3}\right)$ spectra for 8c

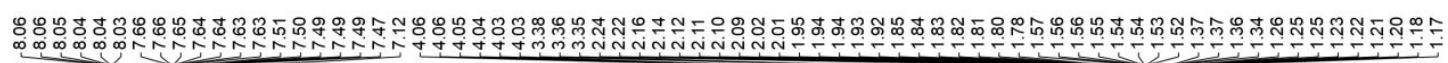
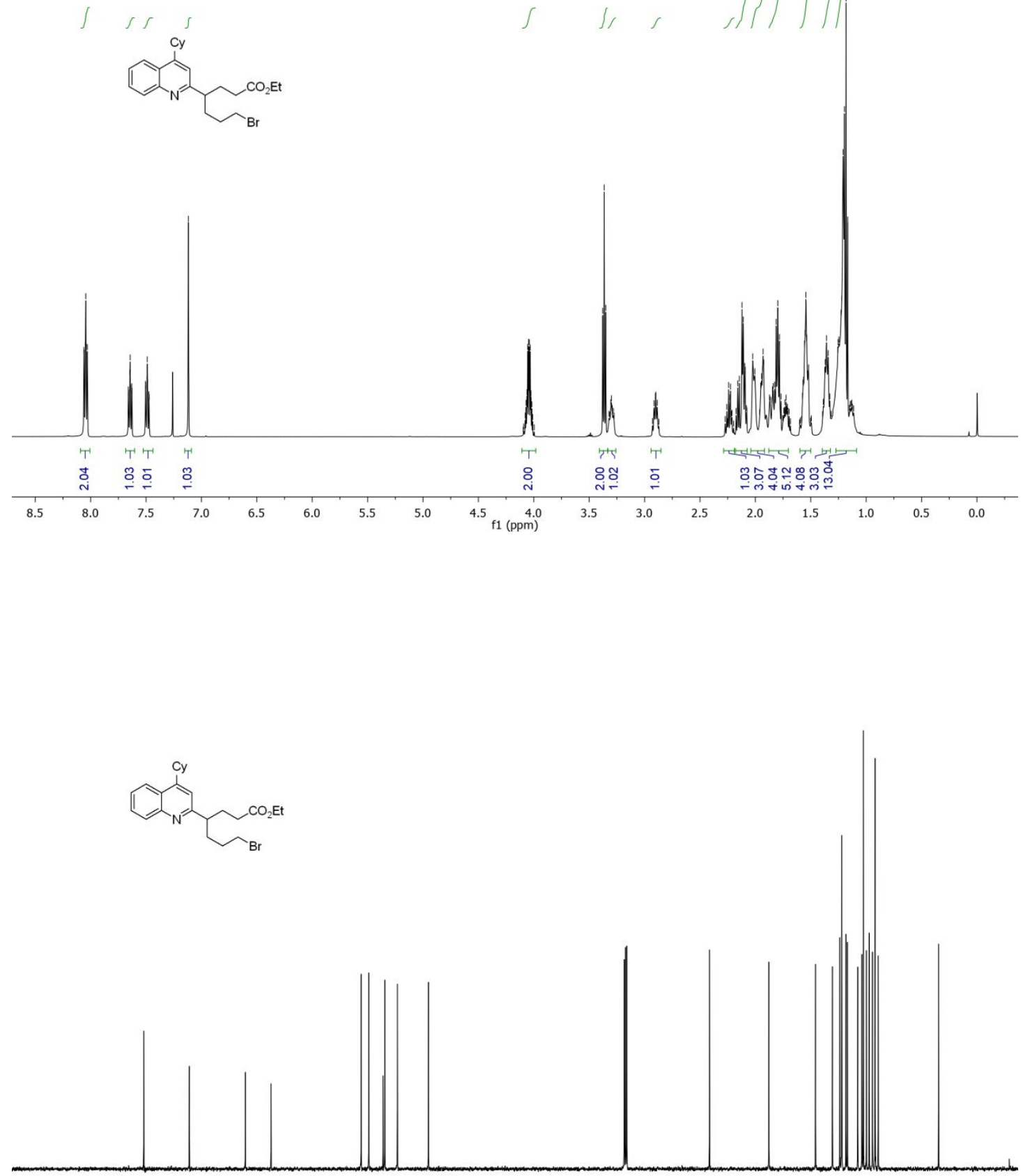

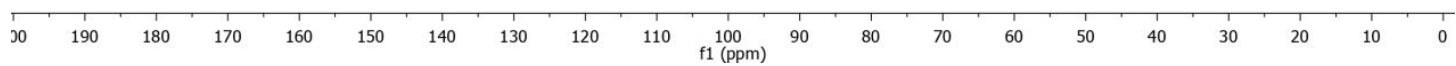


${ }^{1} \mathrm{H}$ NMR (400 MHz, $\left.\mathrm{CDCl}_{3}\right)$ and ${ }^{13} \mathrm{C}$ NMR (125 $\left.\mathrm{MHz}, \mathrm{CDCl}_{3}\right)$ spectra for 8d

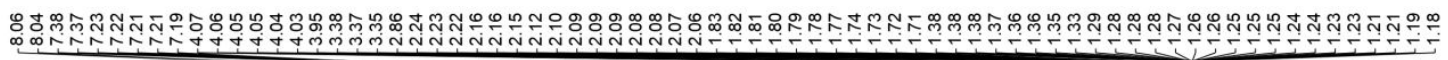
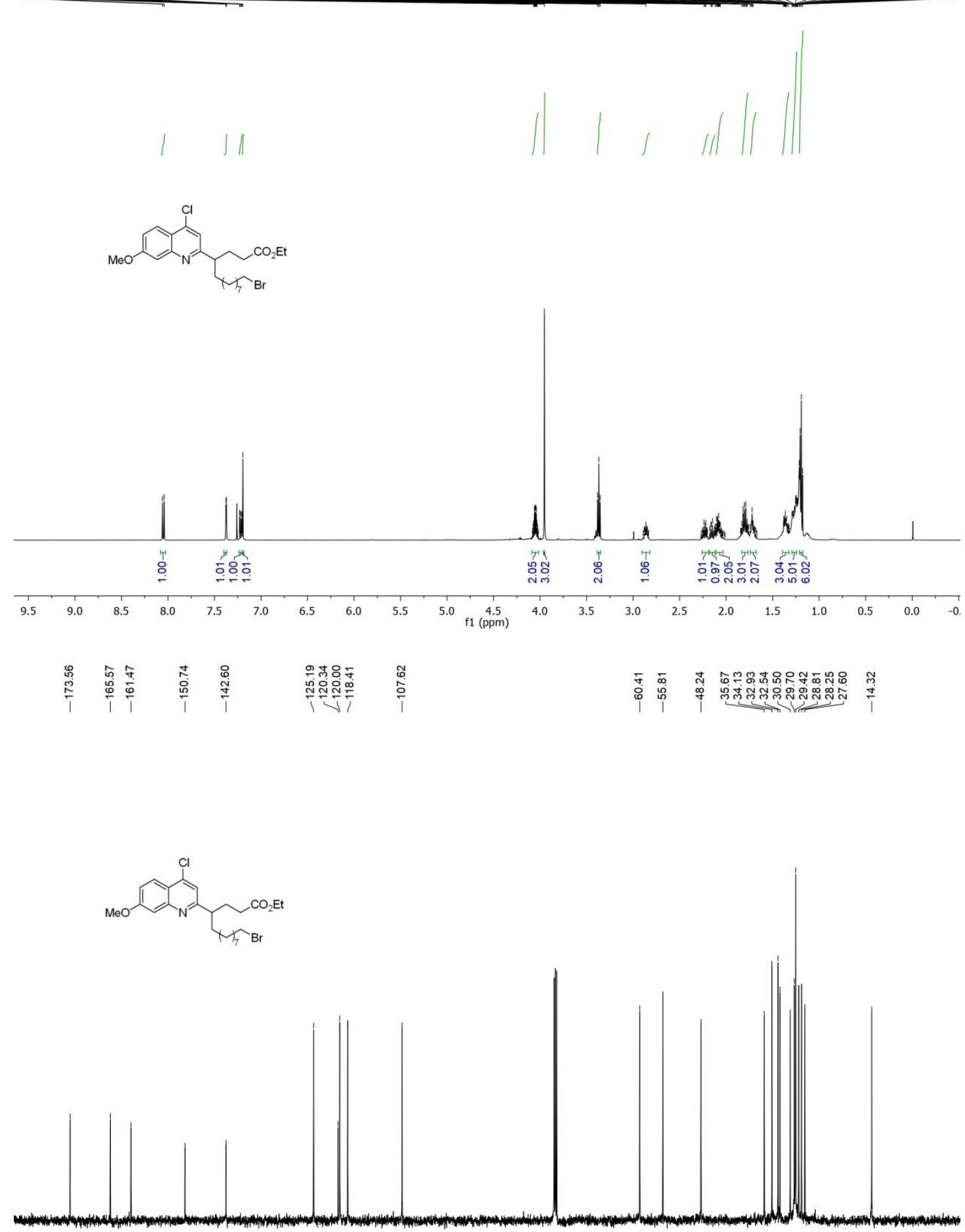

180
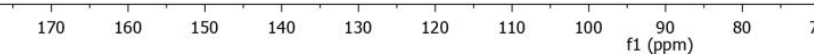

$60 \quad 50$

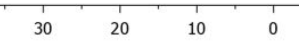


${ }^{1} \mathrm{H}$ NMR (400 MHz, $\left.\mathrm{CDCl}_{3}\right)$ and ${ }^{13} \mathrm{C}$ NMR (125 $\left.\mathrm{MHz}, \mathrm{CDCl}_{3}\right)$ spectra for $8 \mathrm{e}$

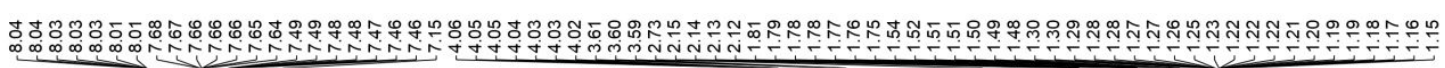
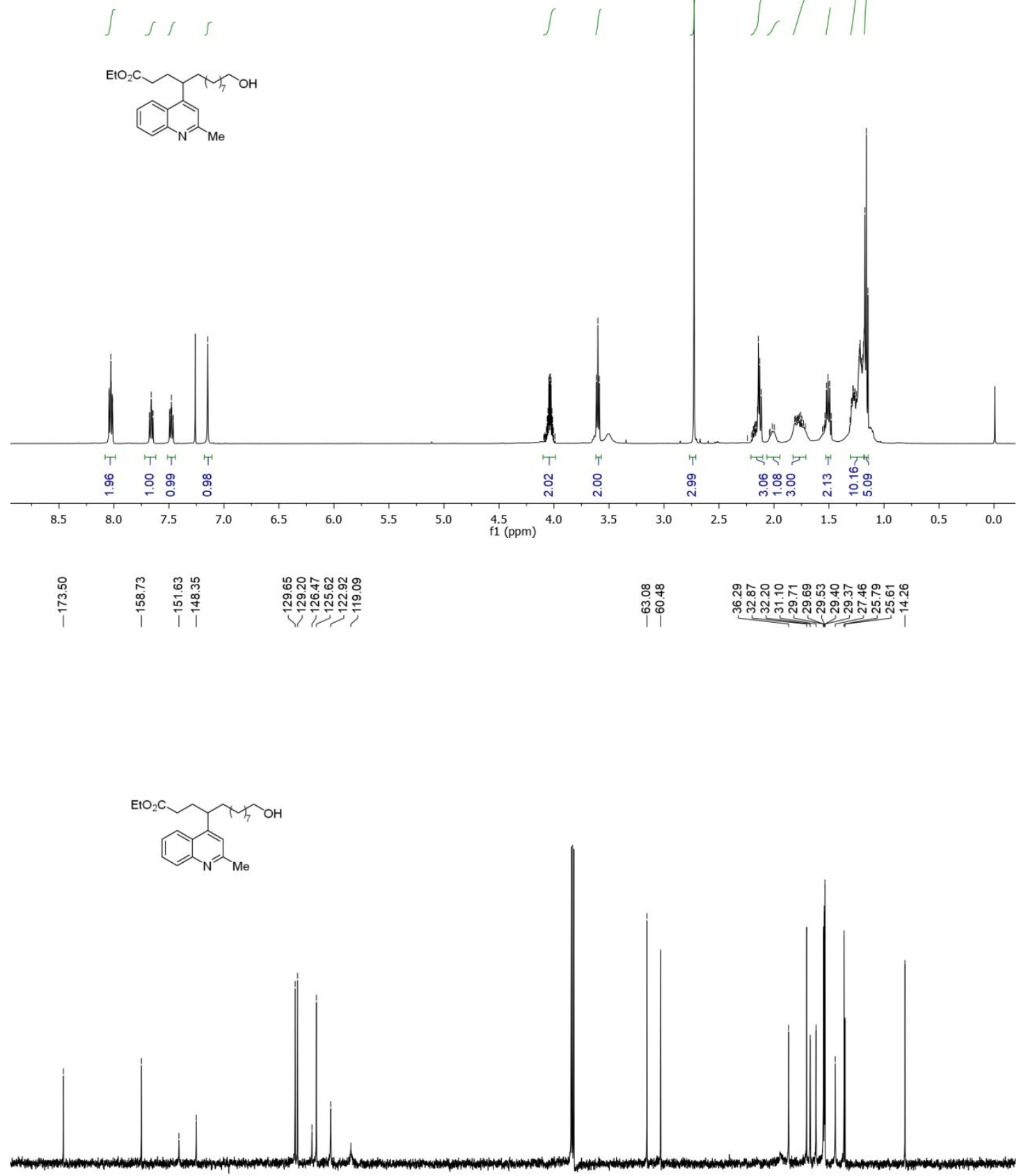

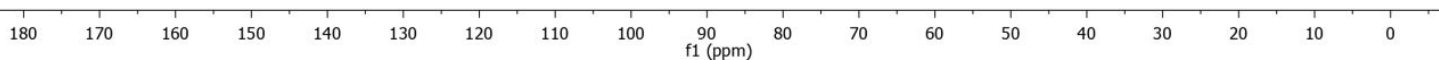


${ }^{1} \mathrm{H}$ NMR (400 MHz, $\left.\mathrm{CDCl}_{3}\right)$ and ${ }^{13} \mathrm{C}$ NMR (125 MHz, $\left.\mathrm{CDCl}_{3}\right)$ spectra for $8 f$

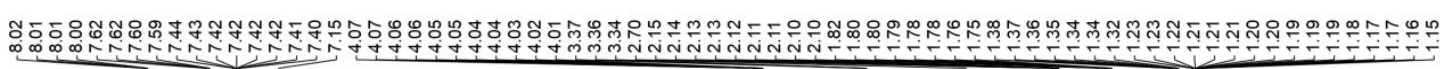
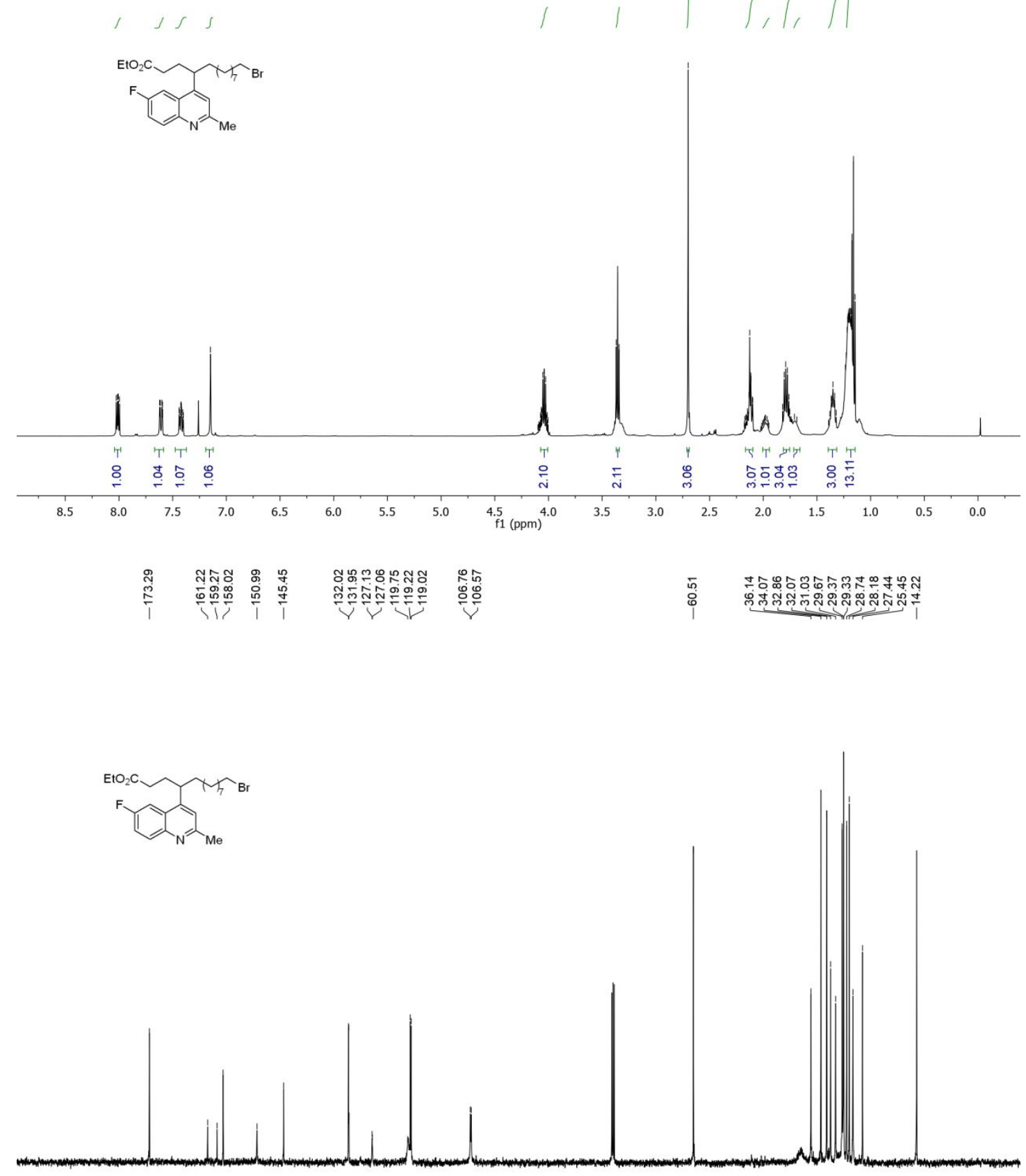

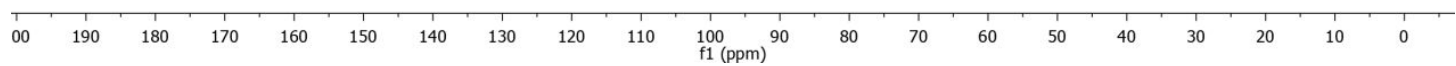


${ }^{1} \mathrm{H}$ NMR (400 MHz, $\mathrm{CDCl}_{3}$ ) and ${ }^{13} \mathrm{C}$ NMR (125 $\left.\mathrm{MHz}, \mathrm{CDCl}_{3}\right)$ spectra for $8 \mathrm{~g}$

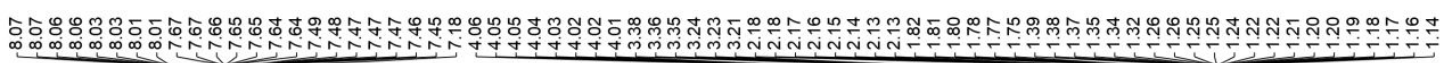
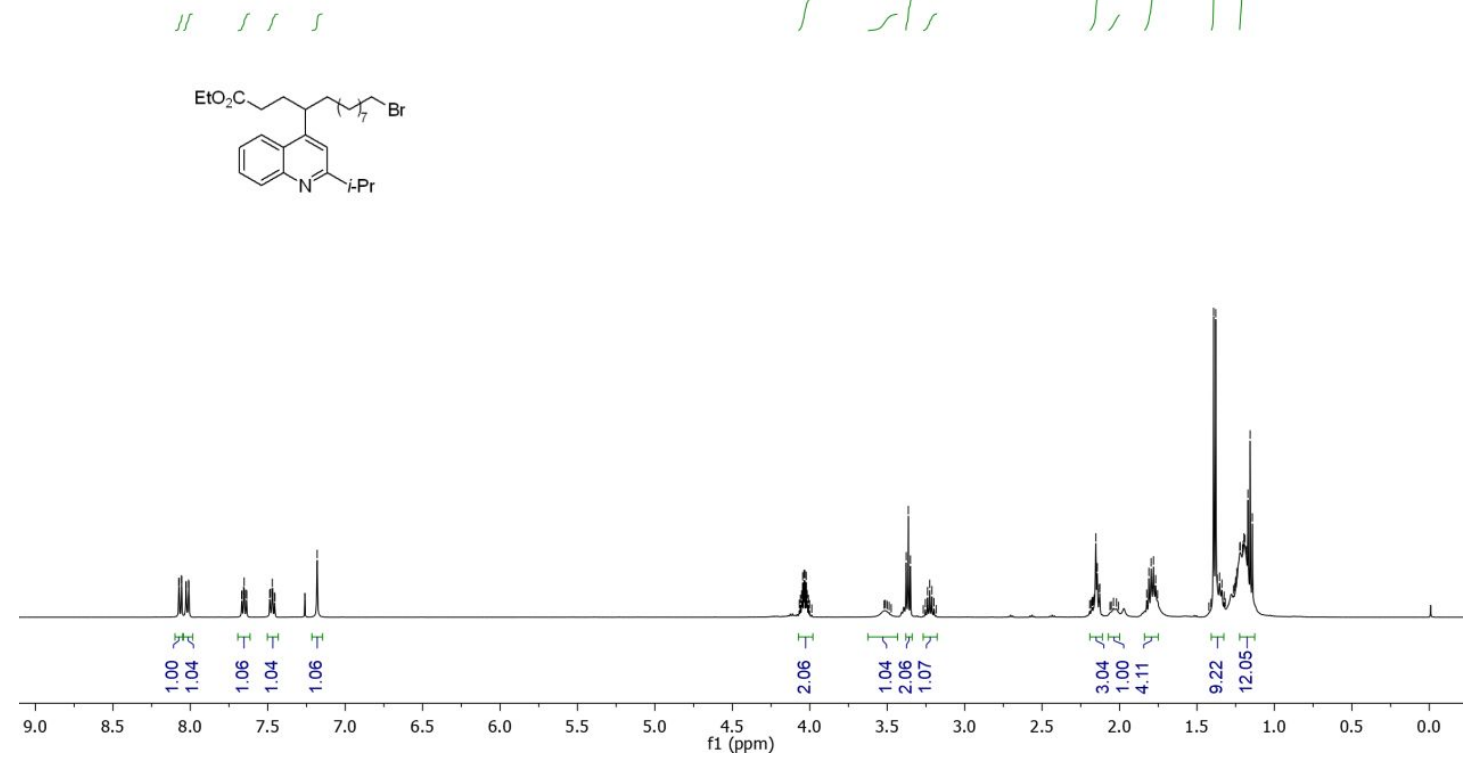

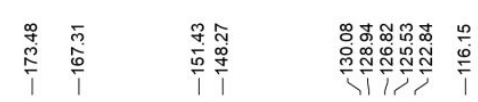

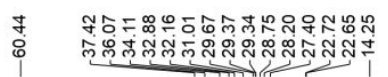
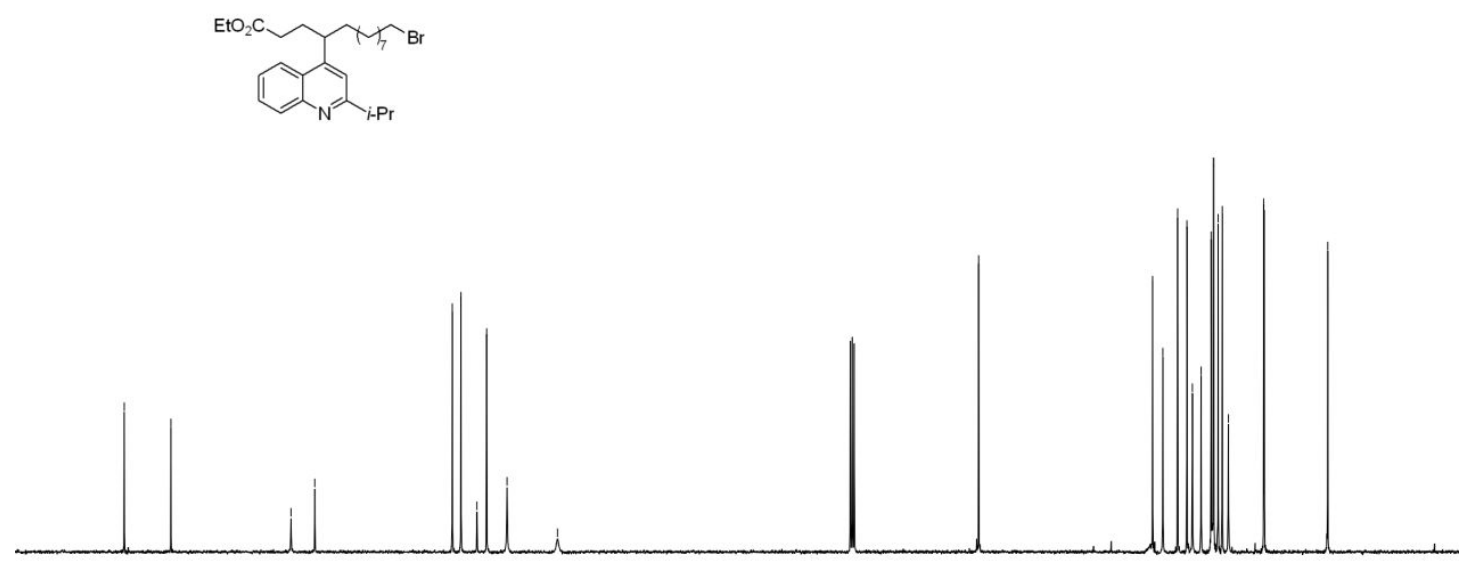

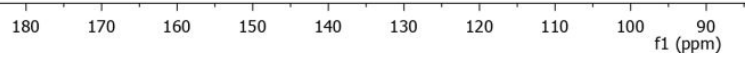


${ }^{1} \mathrm{H}$ NMR (400 MHz, $\left.\mathrm{CDCl}_{3}\right)$ and ${ }^{13} \mathrm{C}$ NMR (125 MHz, $\left.\mathrm{CDCl}_{3}\right)$ spectra for $8 \mathrm{~h}$

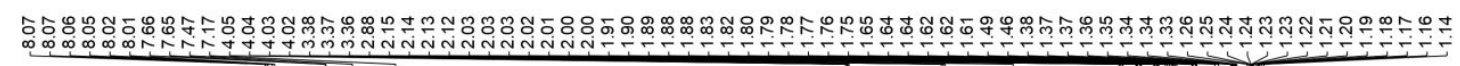

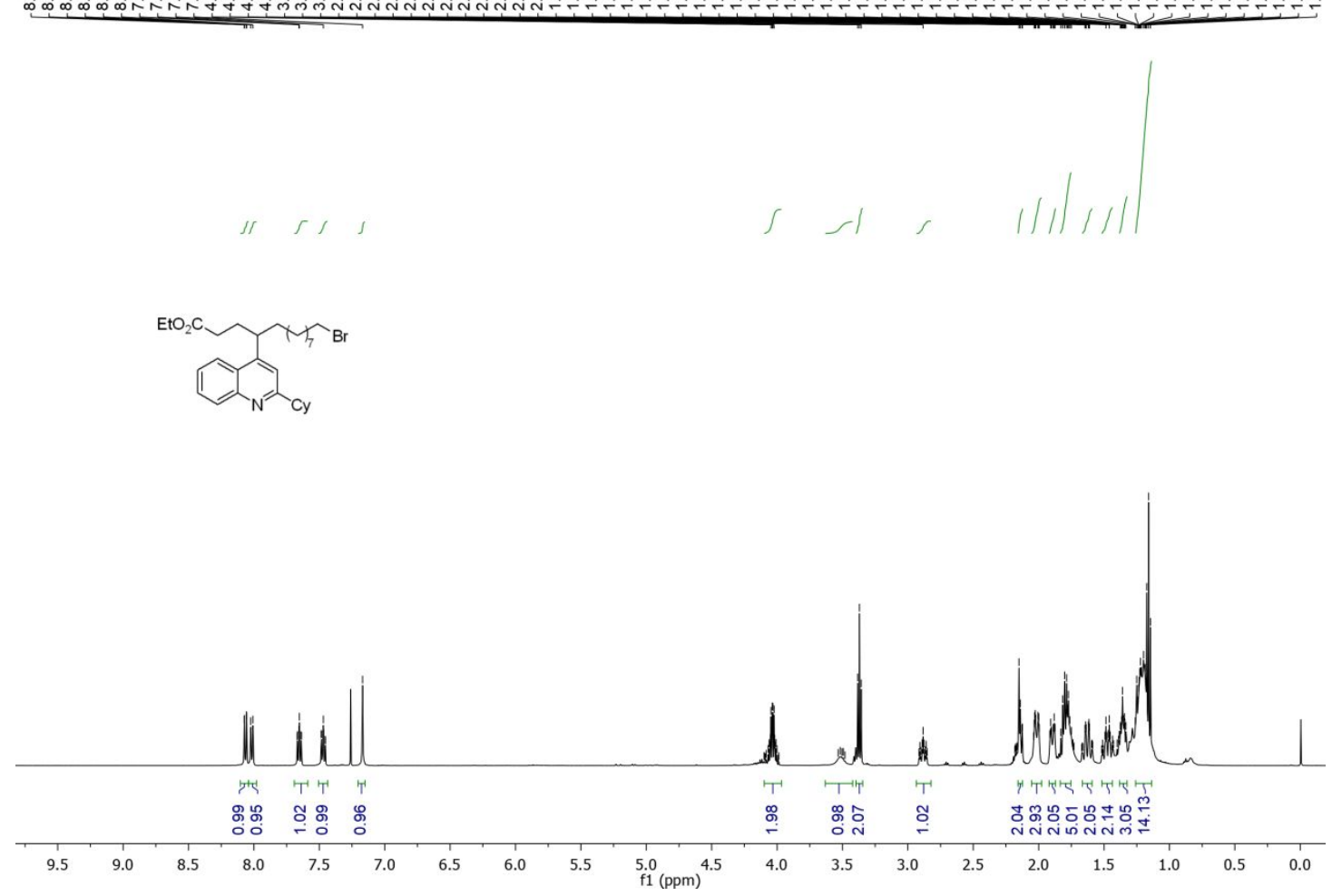

†

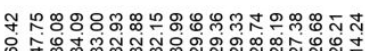

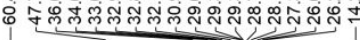

(1)

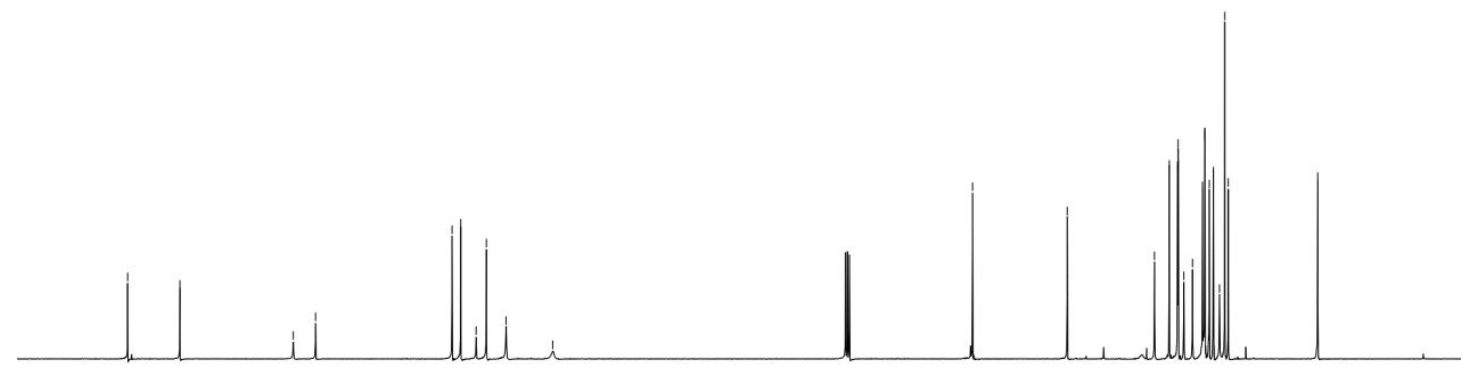

180

$170 \quad 16$

150

140

$130 \quad 12$

$110 \quad 100$

f1 $\left(\begin{array}{c}90 \\ \text { ppm) }\end{array}\right.$ 
${ }^{1} \mathrm{H}$ NMR (400 MHz, $\left.\mathrm{CDCl}_{3}\right)$ and ${ }^{13} \mathrm{C}$ NMR (125 $\left.\mathrm{MHz}, \mathrm{CDCl}_{3}\right)$ spectra for 8i

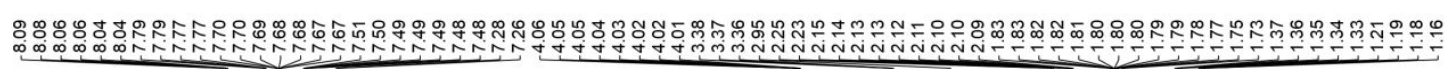
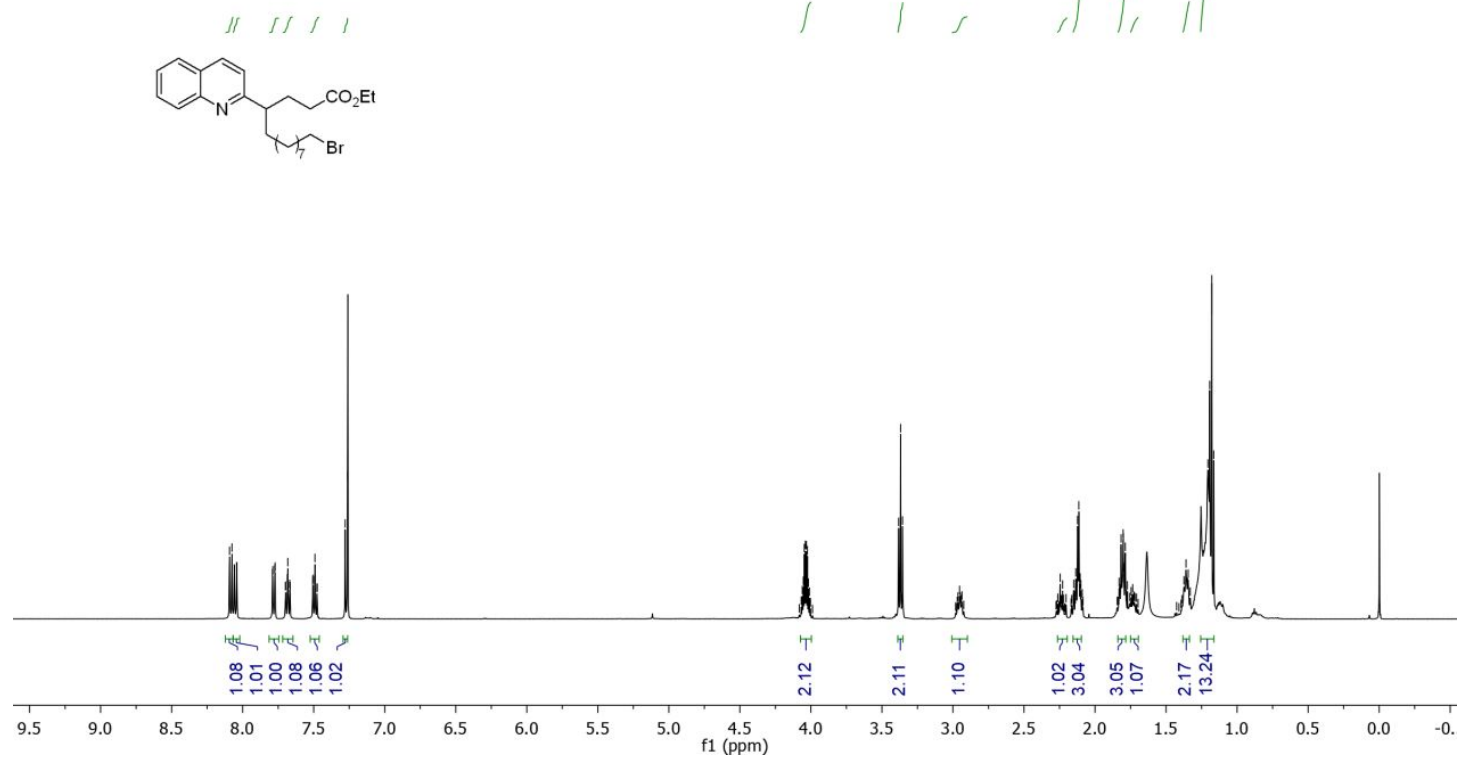

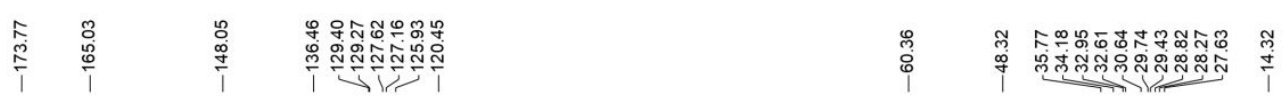

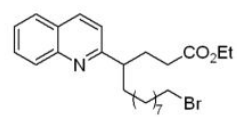

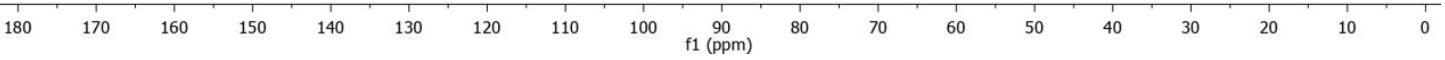


${ }^{1} \mathrm{H}$ NMR (400 MHz, $\left.\mathrm{CDCl}_{3}\right)$ and ${ }^{13} \mathrm{C}$ NMR (125 $\left.\mathrm{MHz}, \mathrm{CDCl}_{3}\right)$ spectra for 8i'

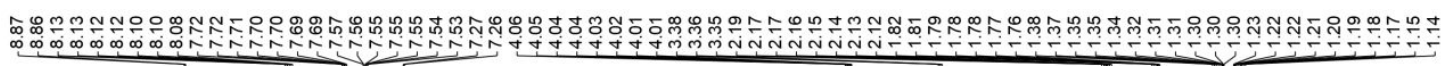
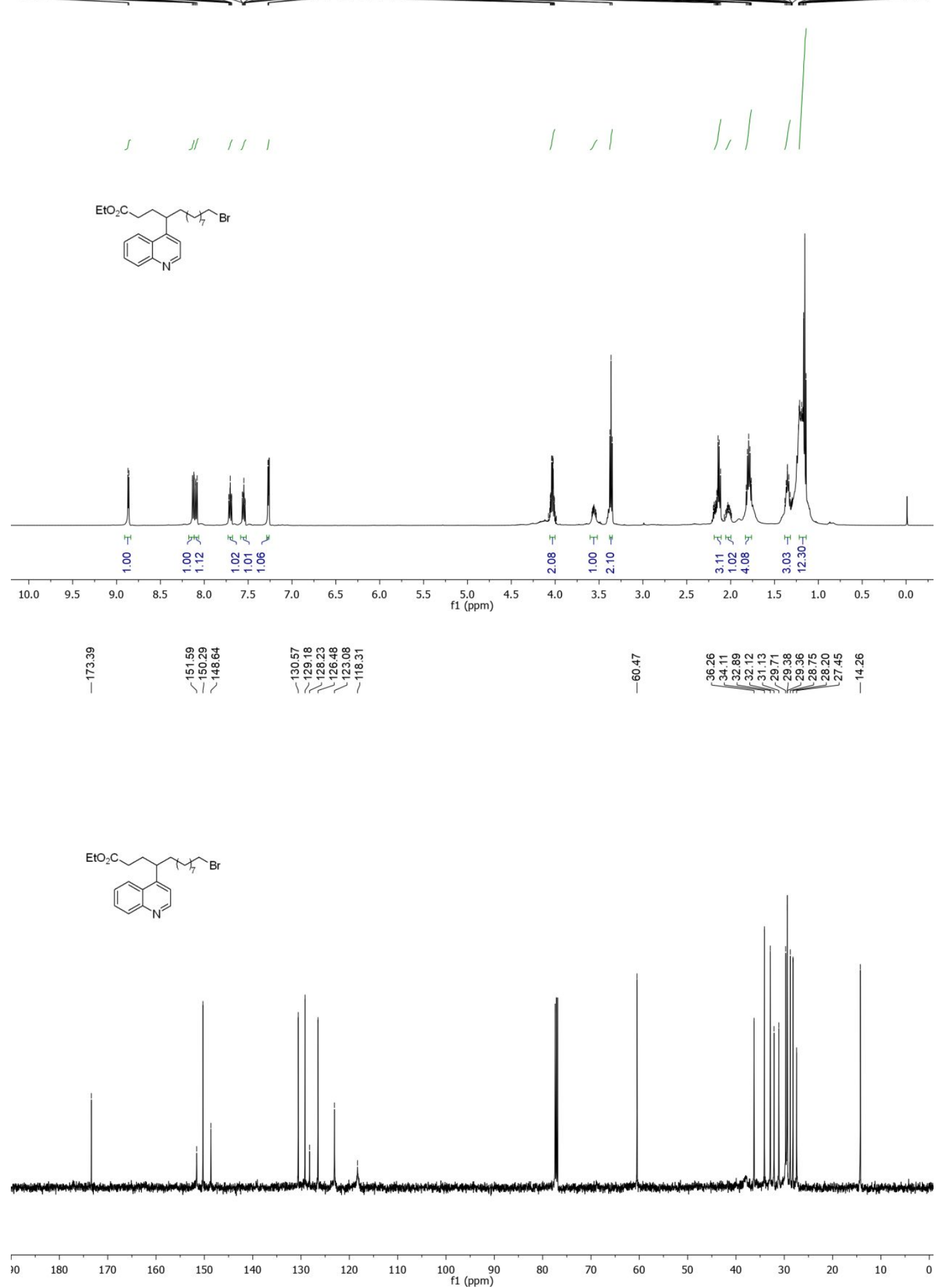
${ }^{1} \mathrm{H}$ NMR (400 MHz, $\left.\mathrm{CDCl}_{3}\right)$ and ${ }^{13} \mathrm{C}$ NMR (125 $\left.\mathrm{MHz}, \mathrm{CDCl}_{3}\right)$ spectra for 8i"

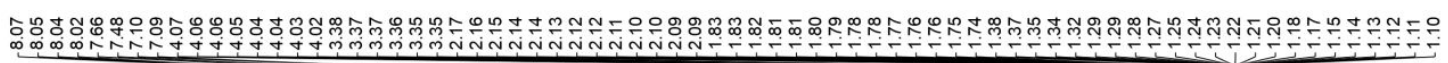

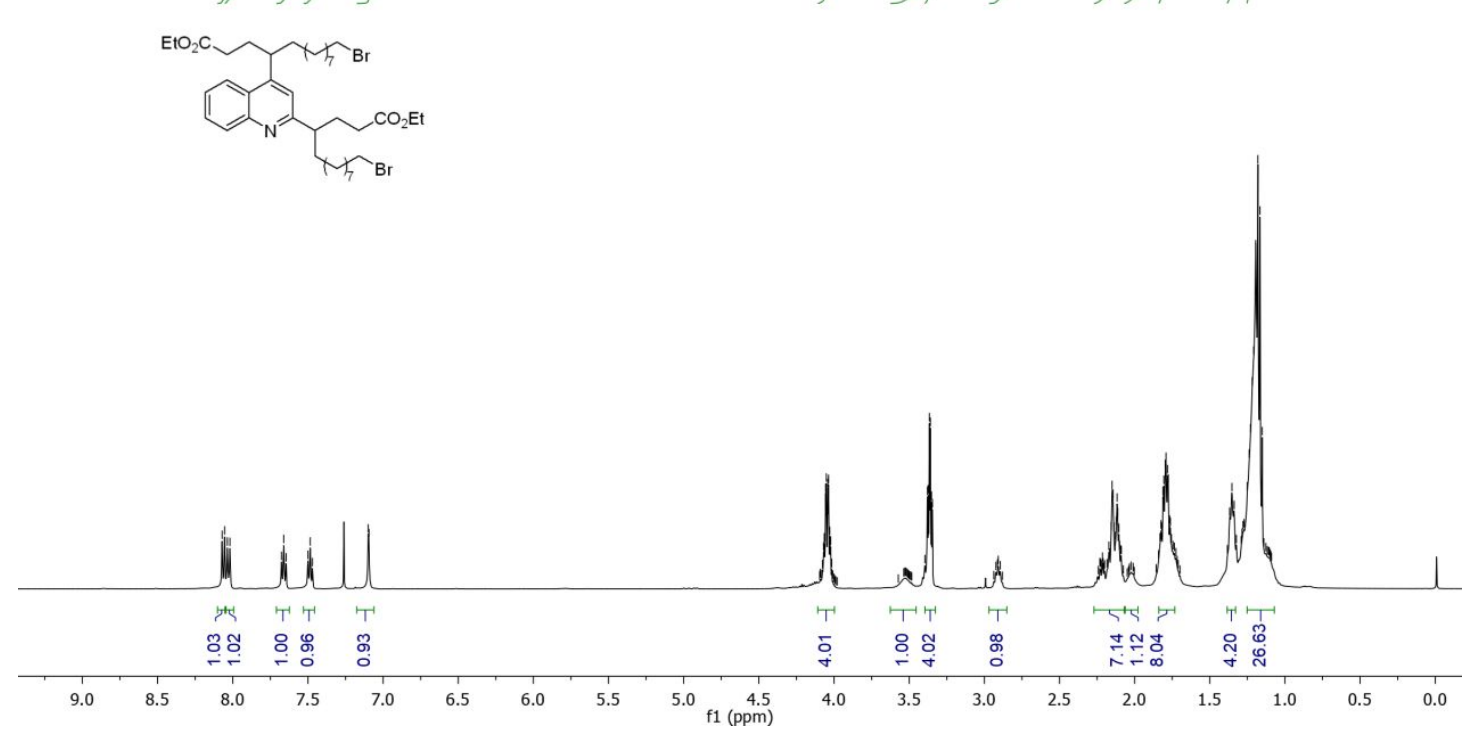

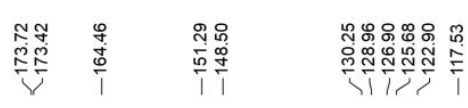

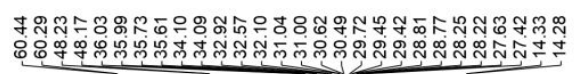
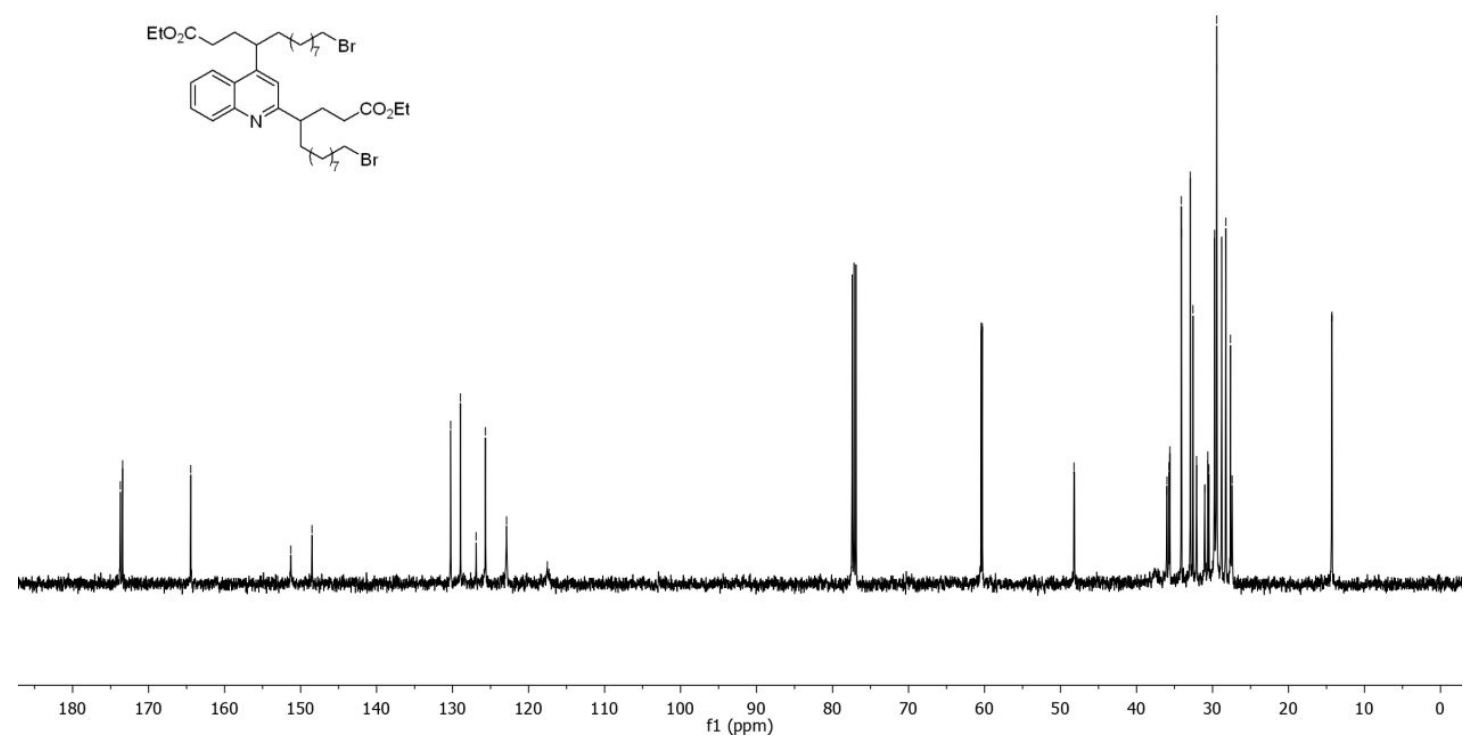
${ }^{1} \mathrm{H}$ NMR (400 MHz, $\left.\mathrm{CDCl}_{3}\right)$ and ${ }^{13} \mathrm{C}$ NMR (125 $\left.\mathrm{MHz}, \mathrm{CDCl}_{3}\right)$ spectra for 8j

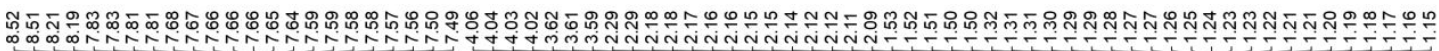
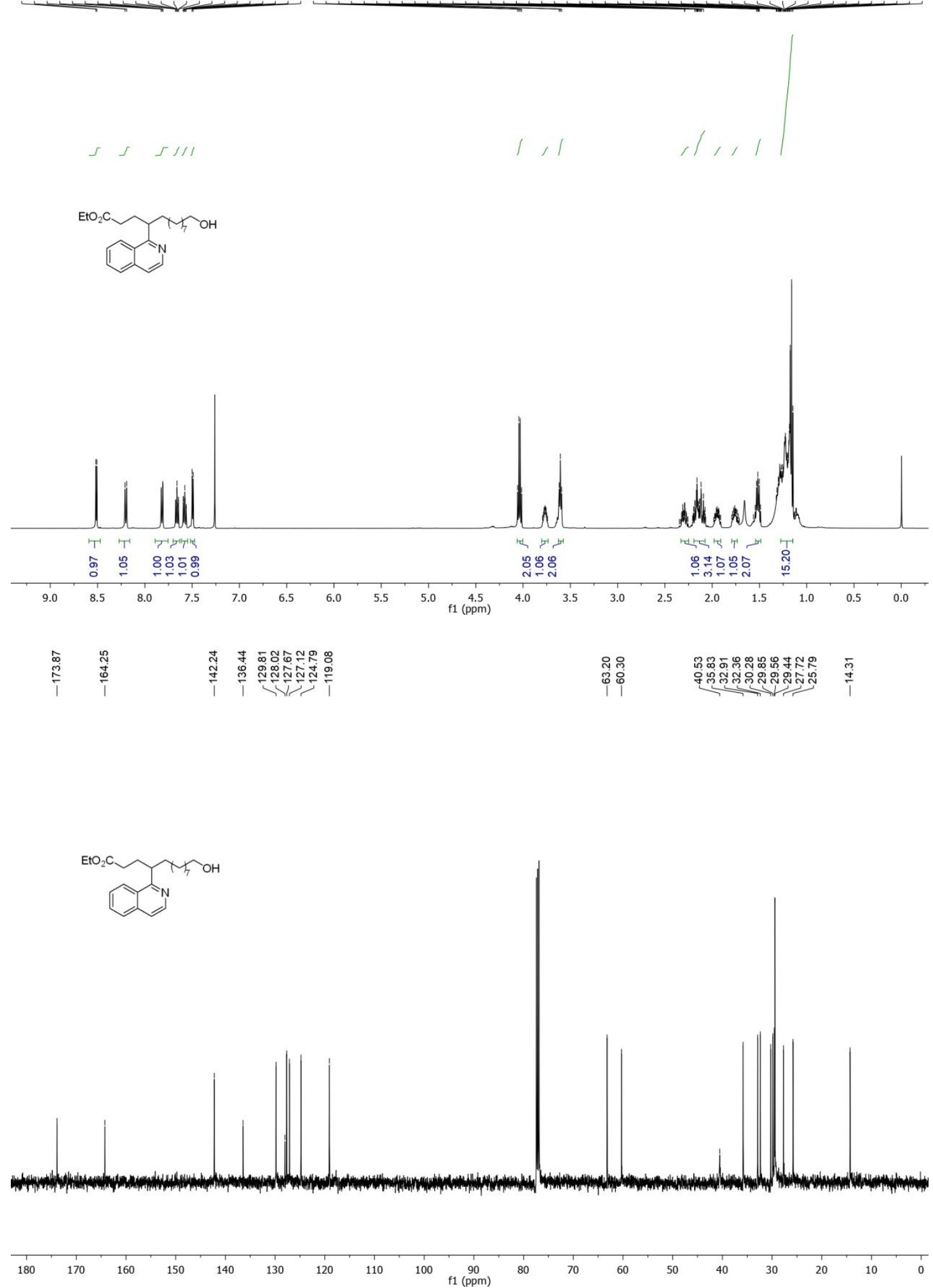
${ }^{1} \mathrm{H}$ NMR (400 MHz, $\left.\mathrm{CDCl}_{3}\right)$ and ${ }^{13} \mathrm{C}$ NMR (125 MHz, $\left.\mathrm{CDCl}_{3}\right)$ spectra for 8k

$\underbrace{0.040}$

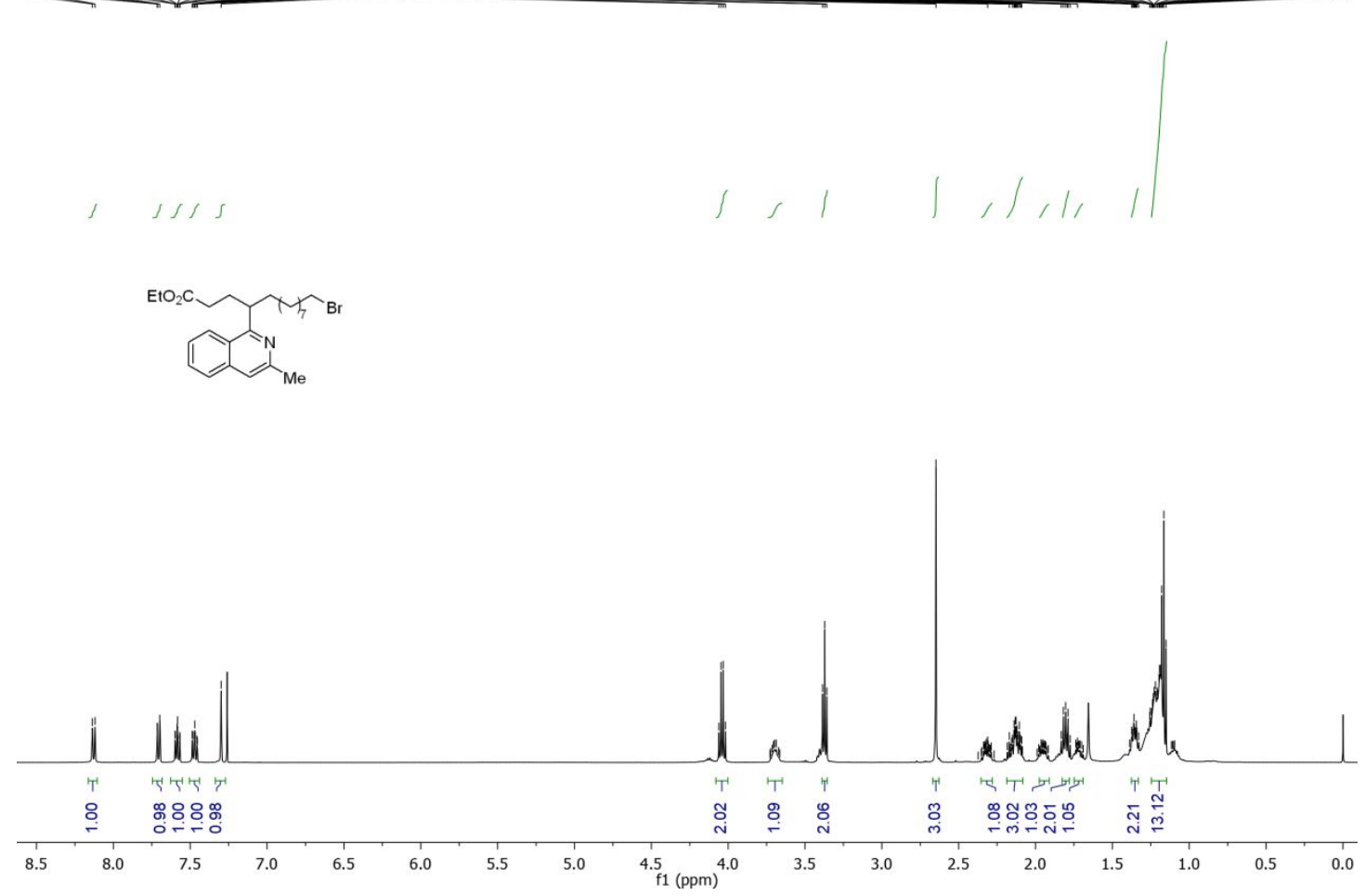

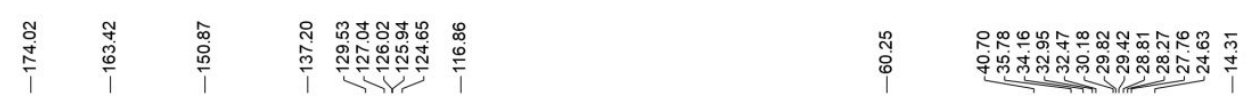

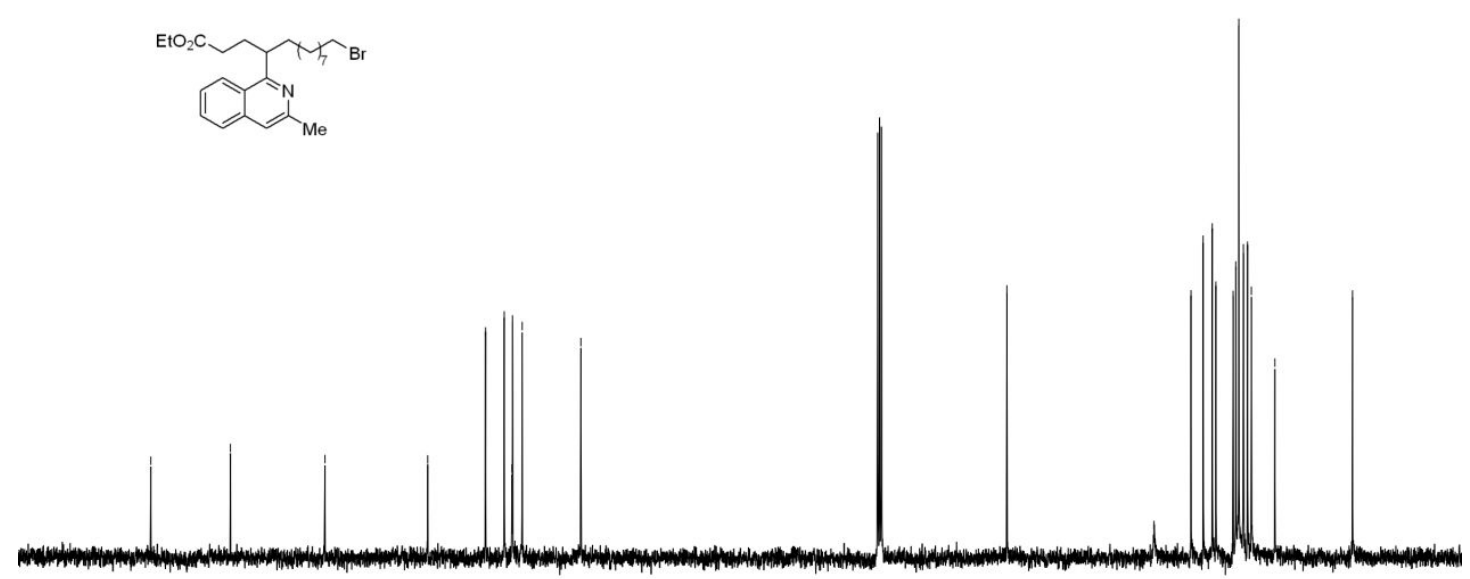

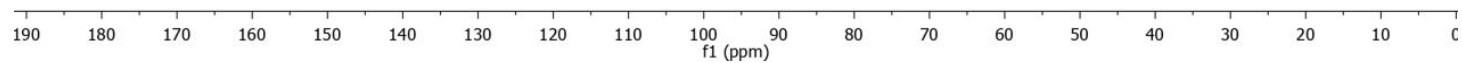


${ }^{1} \mathrm{H}$ NMR (400 MHz, $\left.\mathrm{CDCl}_{3}\right)$ and ${ }^{13} \mathrm{C}$ NMR (125 MHz, $\left.\mathrm{CDCl}_{3}\right)$ spectra for 81

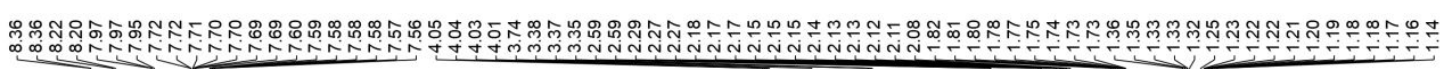

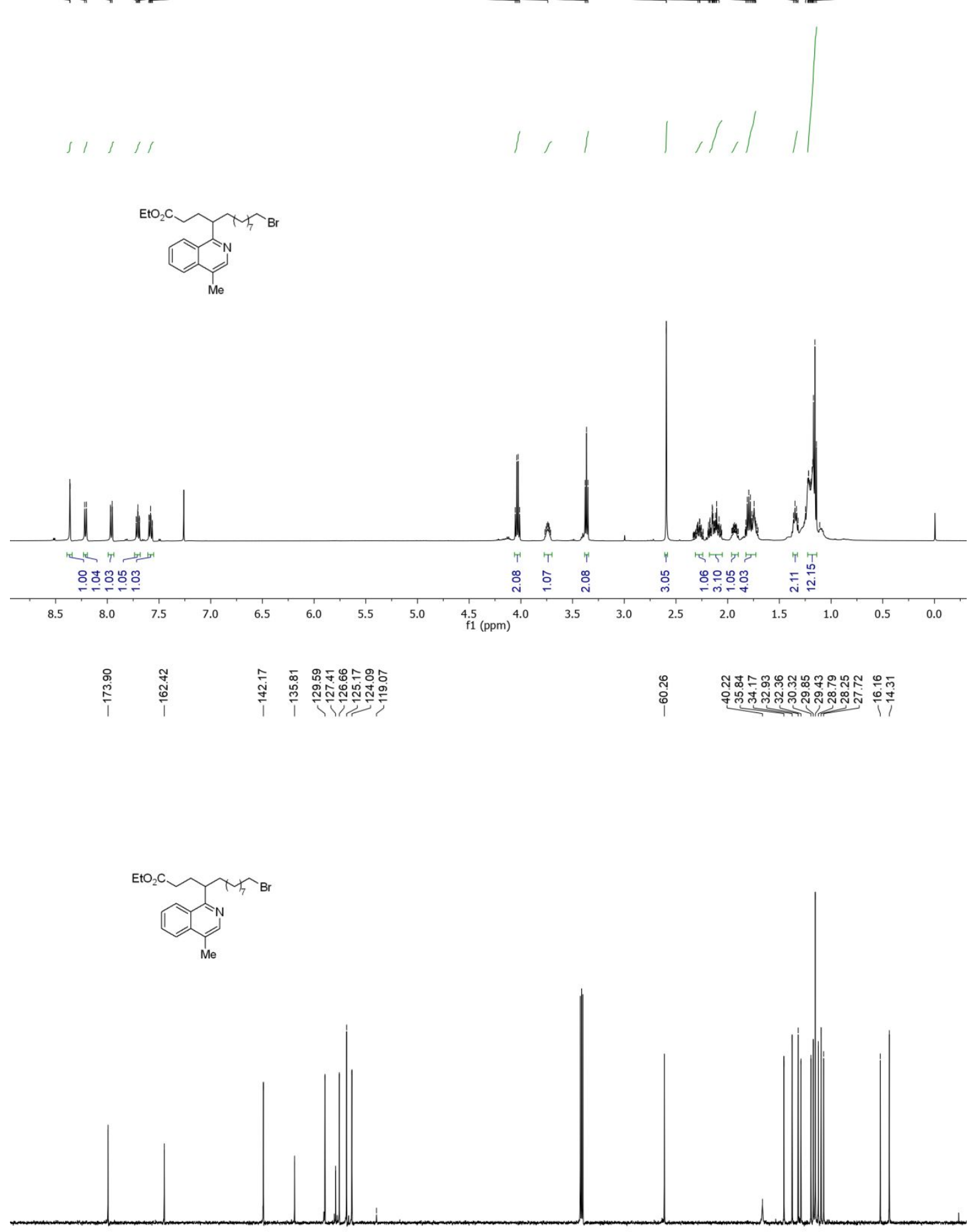

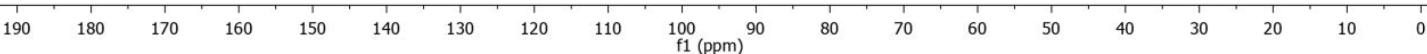


${ }^{1} \mathrm{H}$ NMR (400 MHz, $\left.\mathrm{CDCl}_{3}\right)$ and ${ }^{13} \mathrm{C}$ NMR (125 $\left.\mathrm{MHz}, \mathrm{CDCl}_{3}\right)$ spectra for $8 \mathrm{~m}$

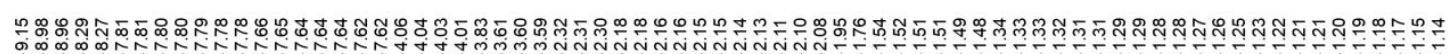
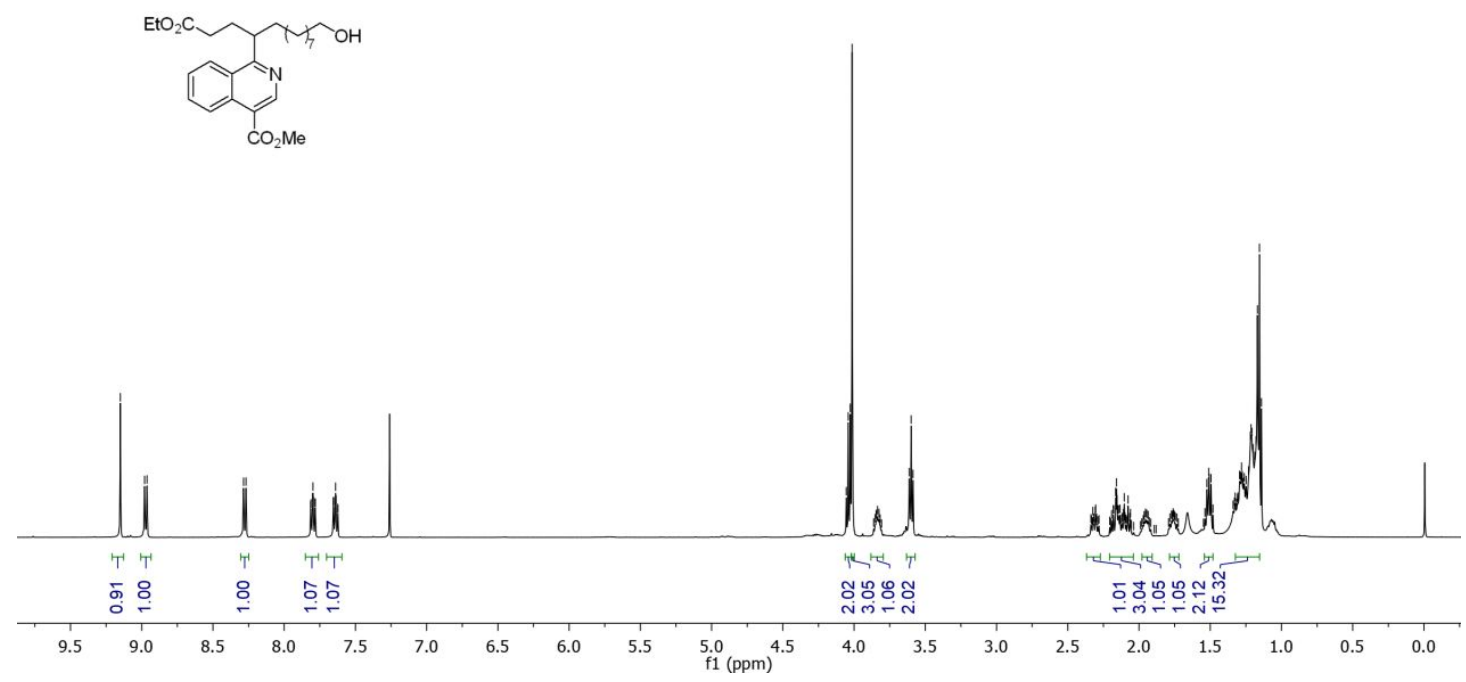

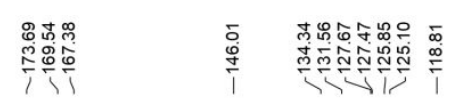

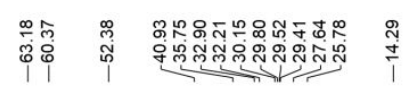

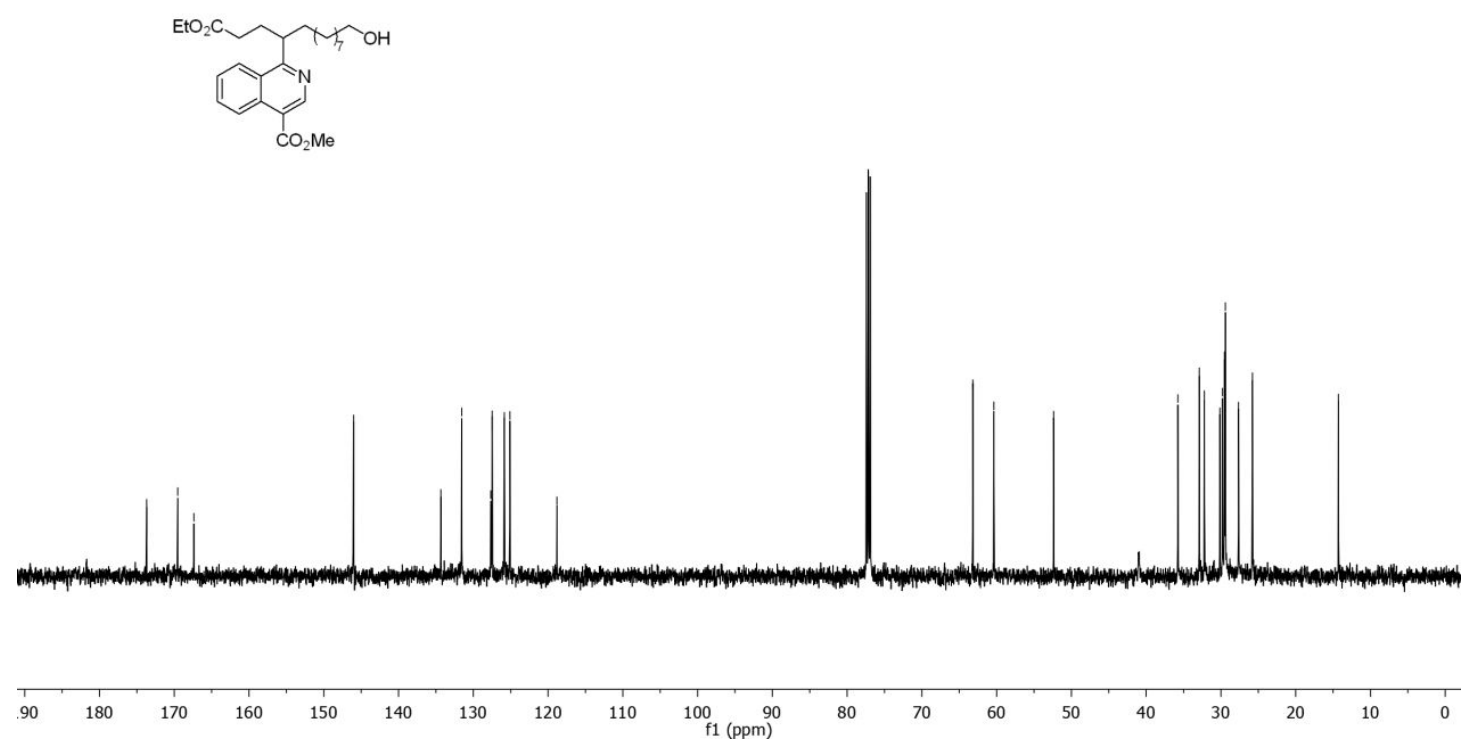

S85 
${ }^{1} \mathrm{H}$ NMR (400 $\left.\mathrm{MHz}, \mathrm{CDCl}_{3}\right)$ and ${ }^{13} \mathrm{C}$ NMR (125 $\left.\mathrm{MHz}, \mathrm{CDCl}_{3}\right)$ spectra for 8n $\underbrace{2}$
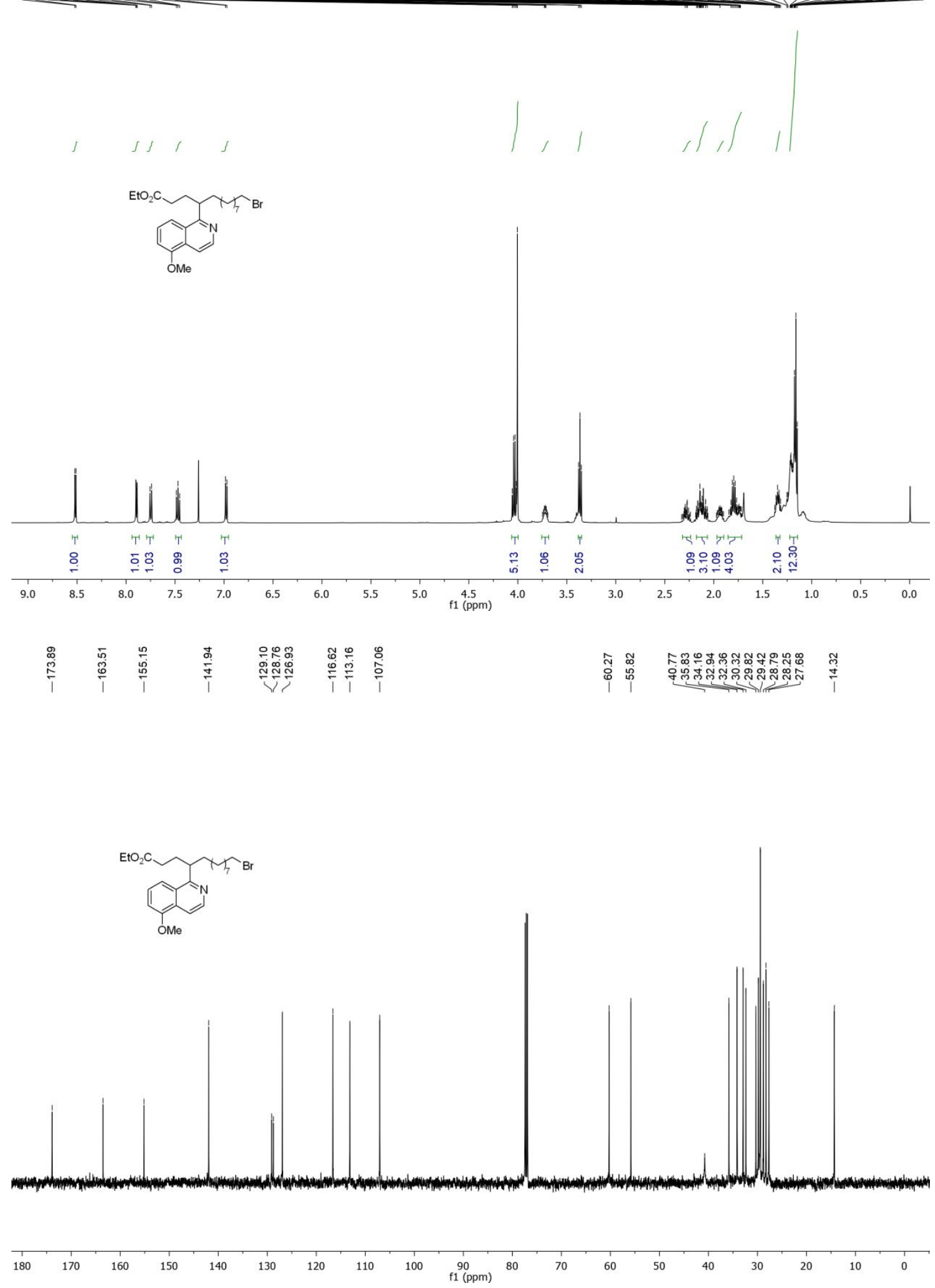
${ }^{1} \mathrm{H}$ NMR (400 $\left.\mathrm{MHz}, \mathrm{CDCl}_{3}\right)$ and ${ }^{13} \mathrm{C}$ NMR (125 $\left.\mathrm{MHz}, \mathrm{CDCl}_{3}\right)$ spectra for 80

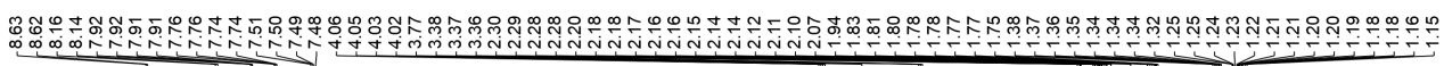
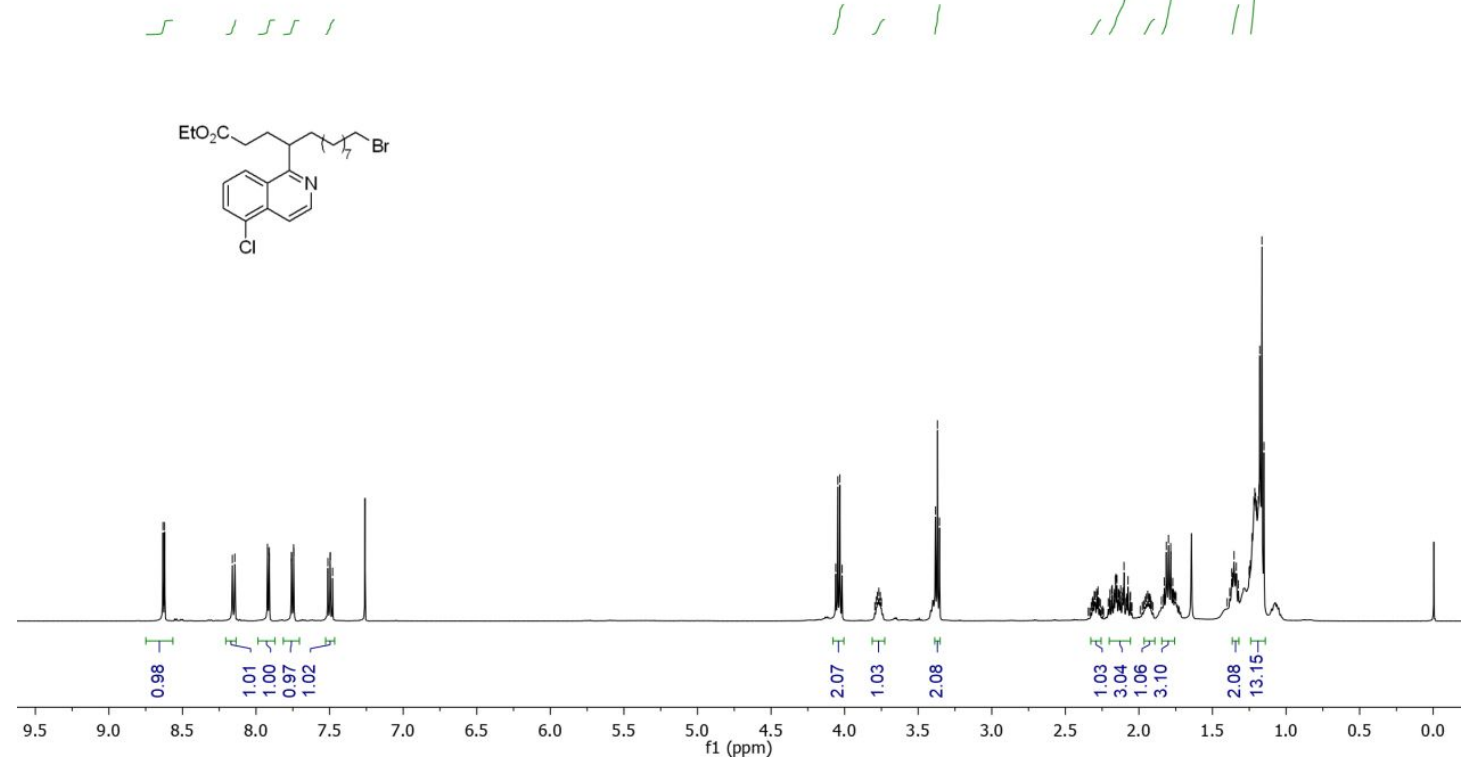

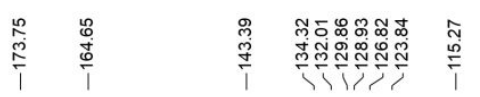

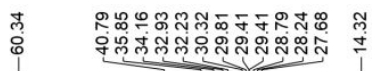

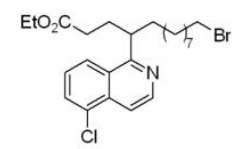

190

180

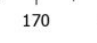

160

140

130

120

${ }_{\mathrm{f} 1(\mathrm{ppm})}^{100}$ 
${ }^{1} \mathrm{H}$ NMR (400 MHz, $\left.\mathrm{CDCl}_{3}\right)$ and ${ }^{13} \mathrm{C}$ NMR (125 MHz, $\left.\mathrm{CDCl}_{3}\right)$ spectra for 8p

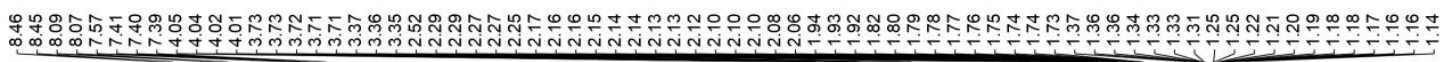
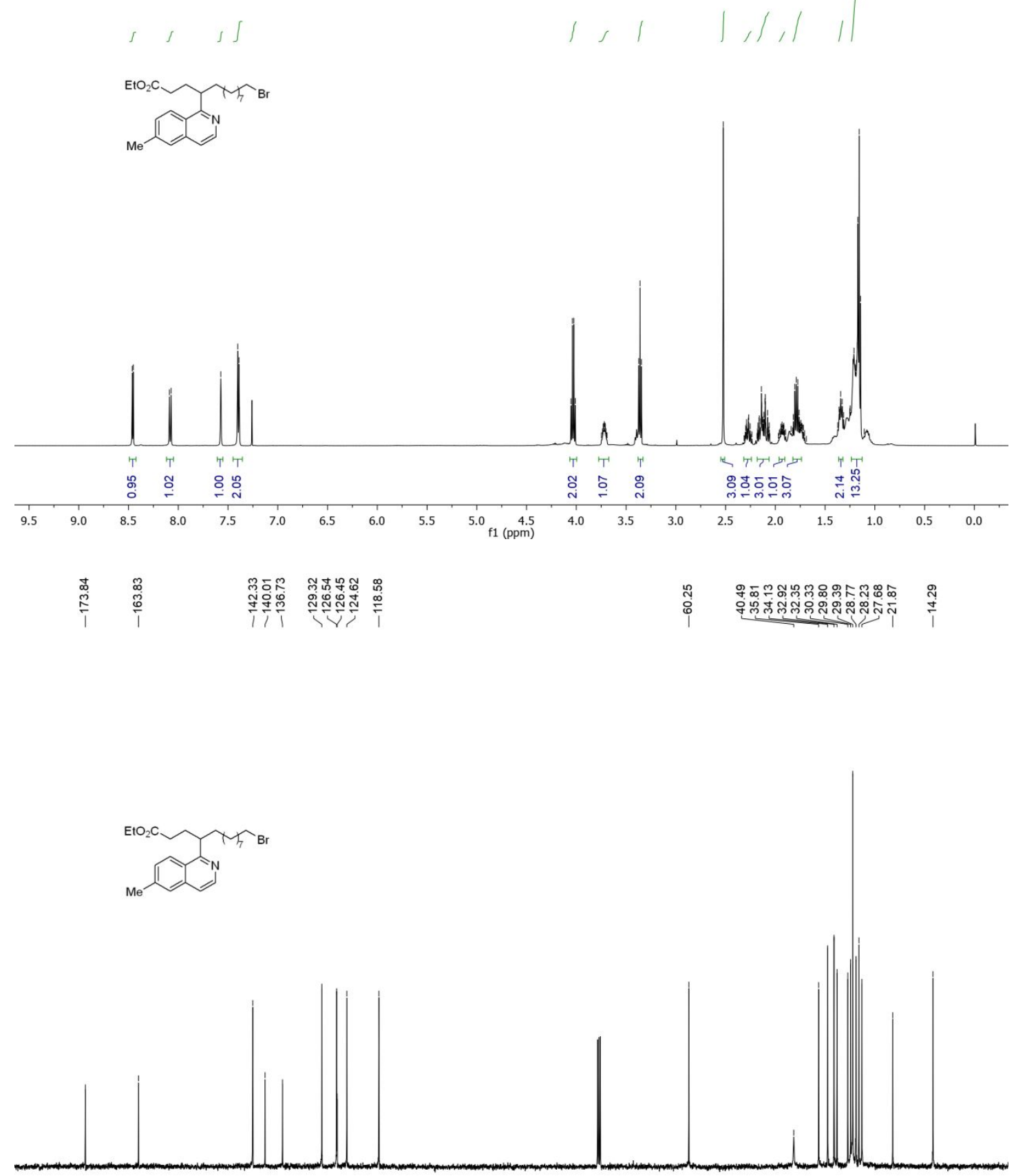

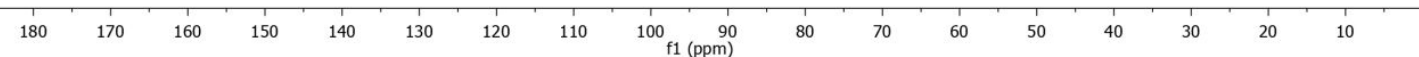


${ }^{1} \mathrm{H}$ NMR (400 $\left.\mathrm{MHz}, \mathrm{CDCl}_{3}\right)$ and ${ }^{13} \mathrm{C}$ NMR (125 $\left.\mathrm{MHz}, \mathrm{CDCl}_{3}\right)$ spectra for 8q

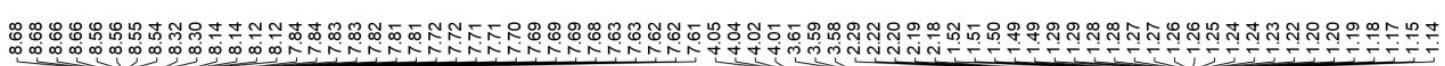

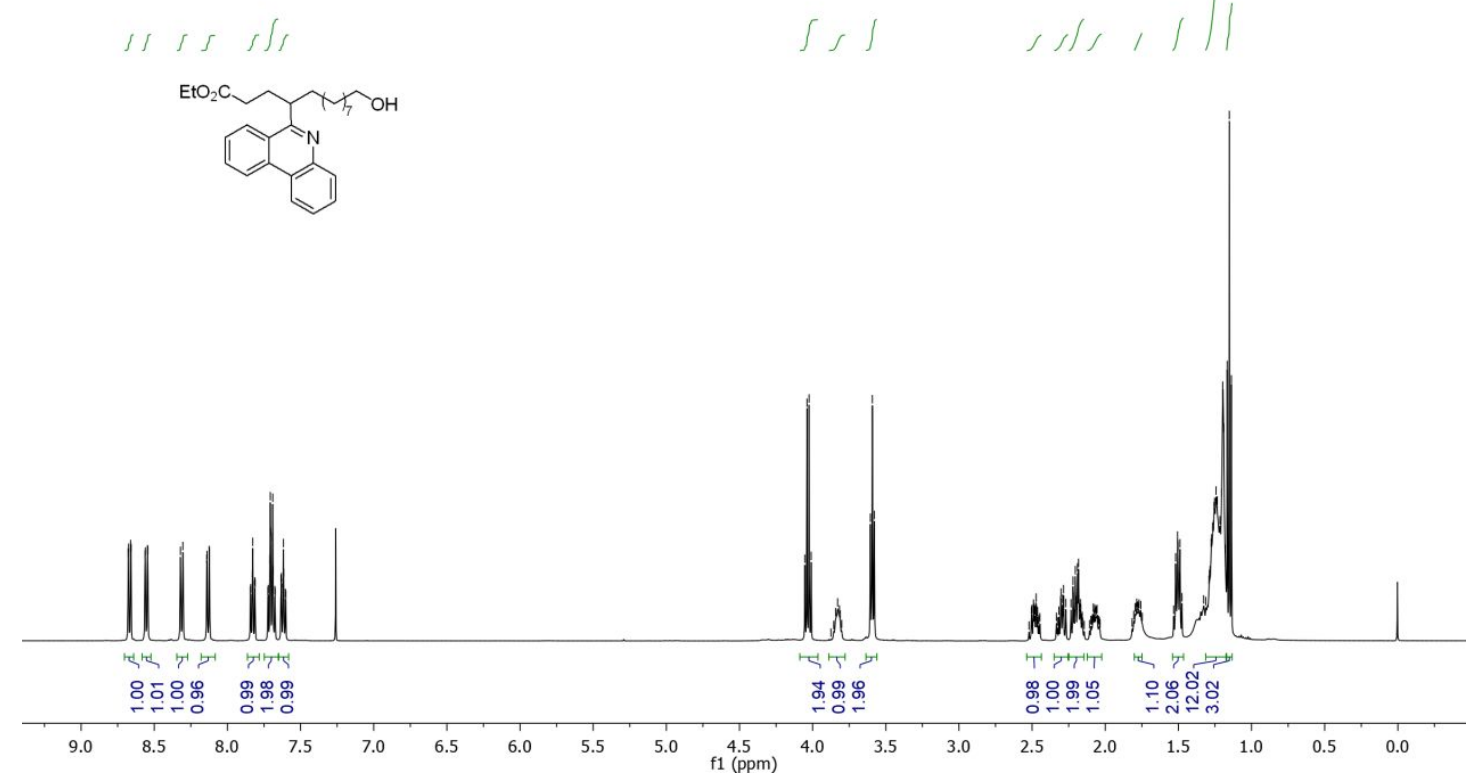

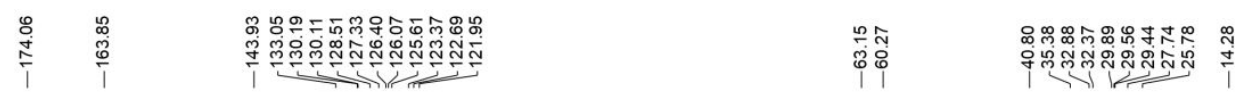

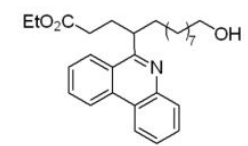

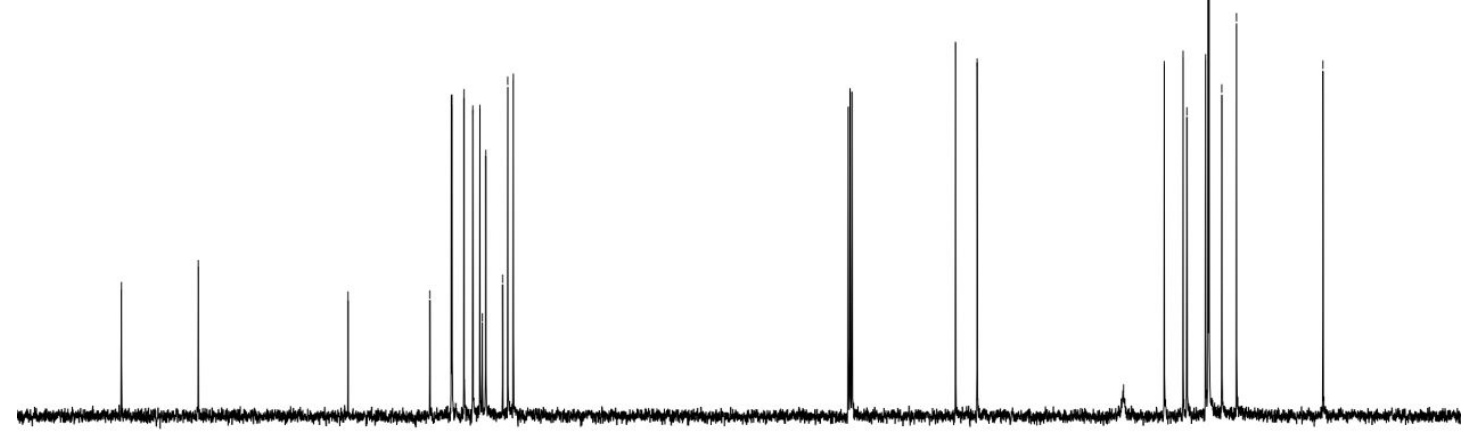

$\begin{array}{lllllllll}180 & 170 & 160 & 150 & 140 & 130 & 120 & 110 & 100 \\ \mathrm{f} 1(\mathrm{ppm})\end{array}$ 
${ }^{1} \mathrm{H}$ NMR (400 MHz, $\left.\mathrm{CDCl}_{3}\right)$ and ${ }^{13} \mathrm{C}$ NMR (125 MHz, $\left.\mathrm{CDCl}_{3}\right)$ spectra for 8r

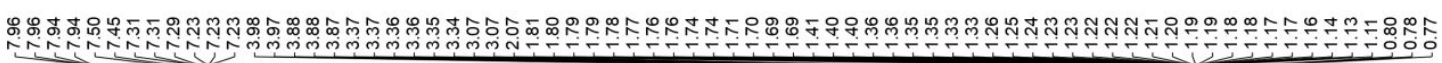
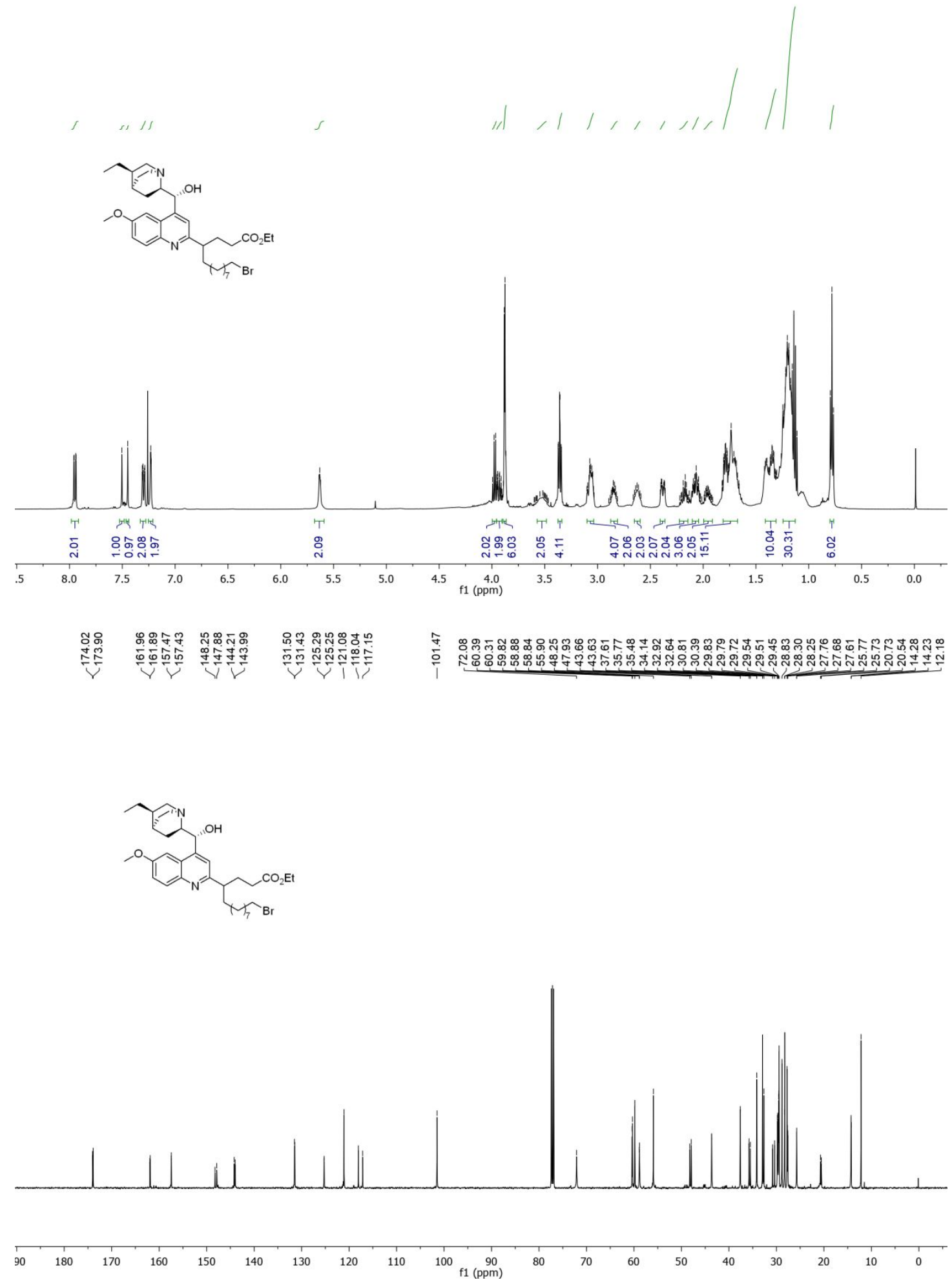
${ }^{1} \mathrm{H}$ NMR (500 MHz, $\left.\mathrm{CDCl}_{3}\right)$ and ${ }^{13} \mathrm{C}$ NMR (125 MHz, $\left.\mathrm{CDCl}_{3}\right)$ spectra for 8s

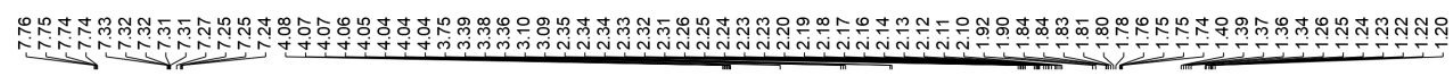

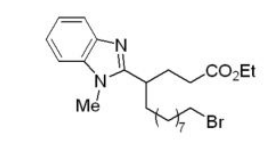

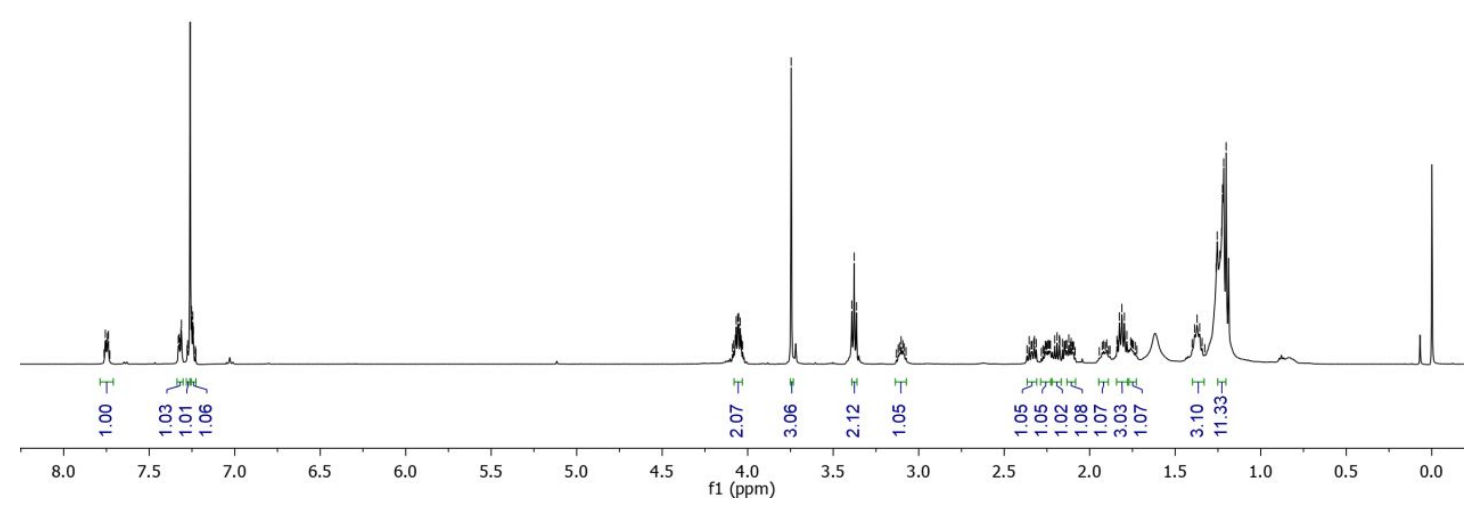

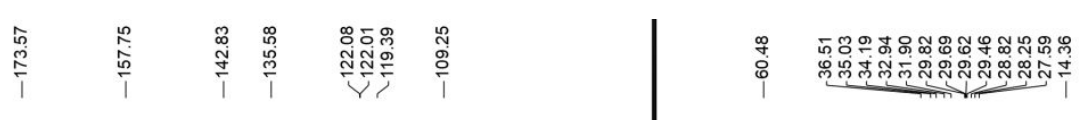

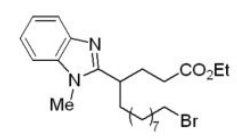

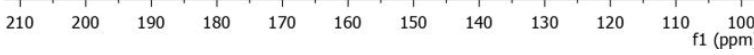


${ }^{1} \mathrm{H}$ NMR (500 MHz, $\left.\mathrm{CDCl}_{3}\right)$ and ${ }^{13} \mathrm{C}$ NMR (125 MHz, $\left.\mathrm{CDCl}_{3}\right)$ spectra for 8t

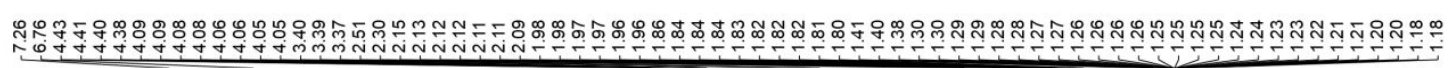

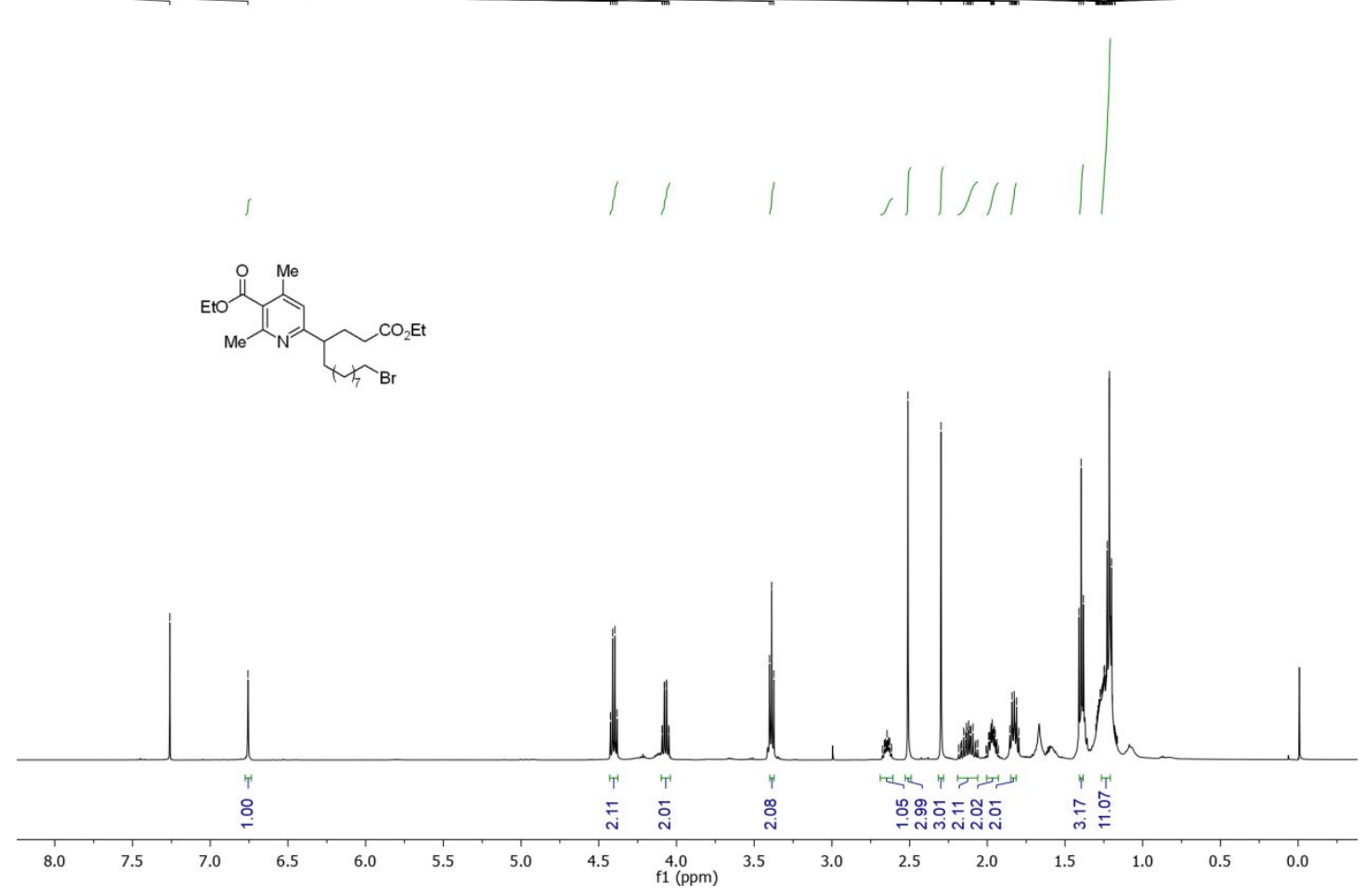

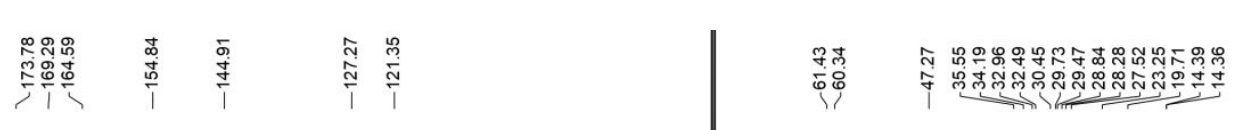
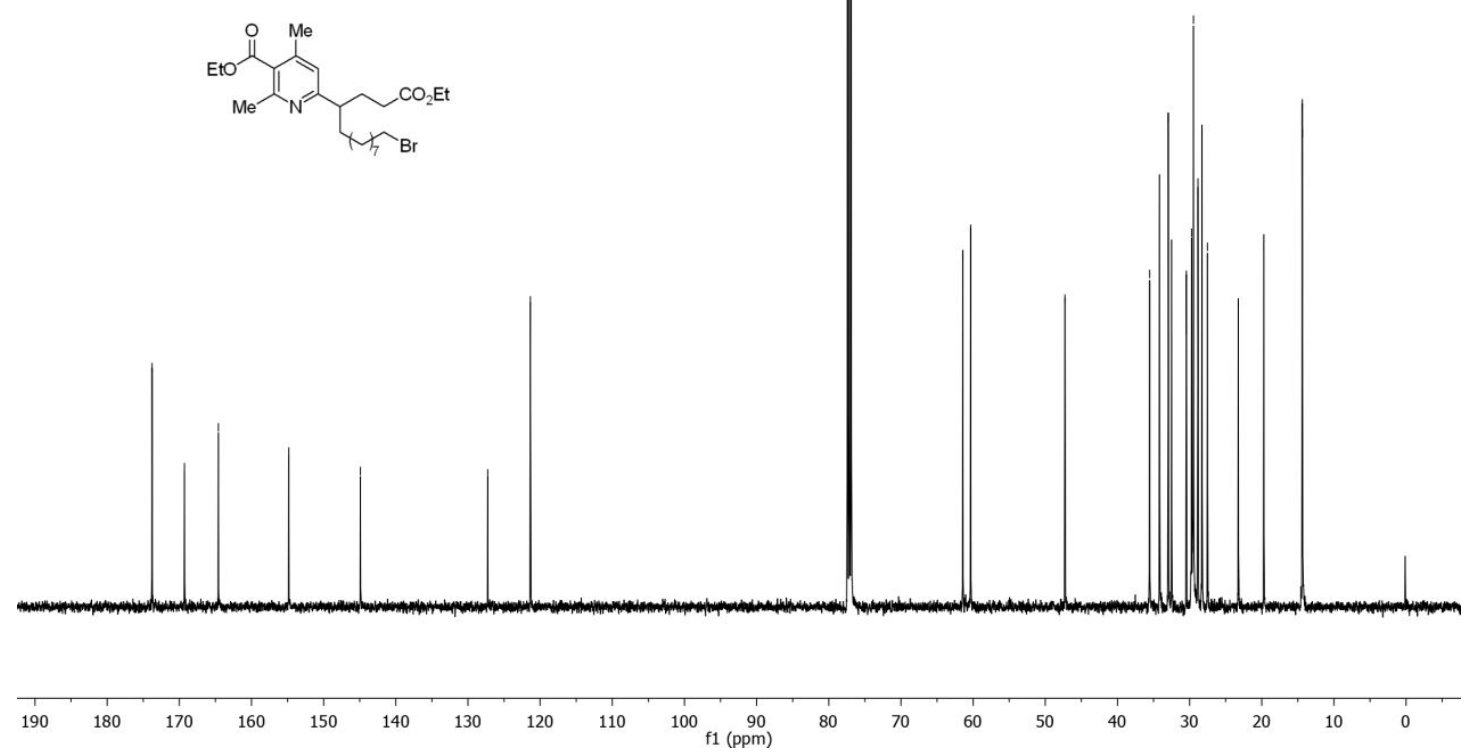
${ }^{1} \mathrm{H}$ NMR (400 MHz, $\left.\mathrm{CDCl}_{3}\right)$ and ${ }^{13} \mathrm{C}$ NMR (125 $\left.\mathrm{MHz}, \mathrm{CDCl}_{3}\right)$ spectra for 9

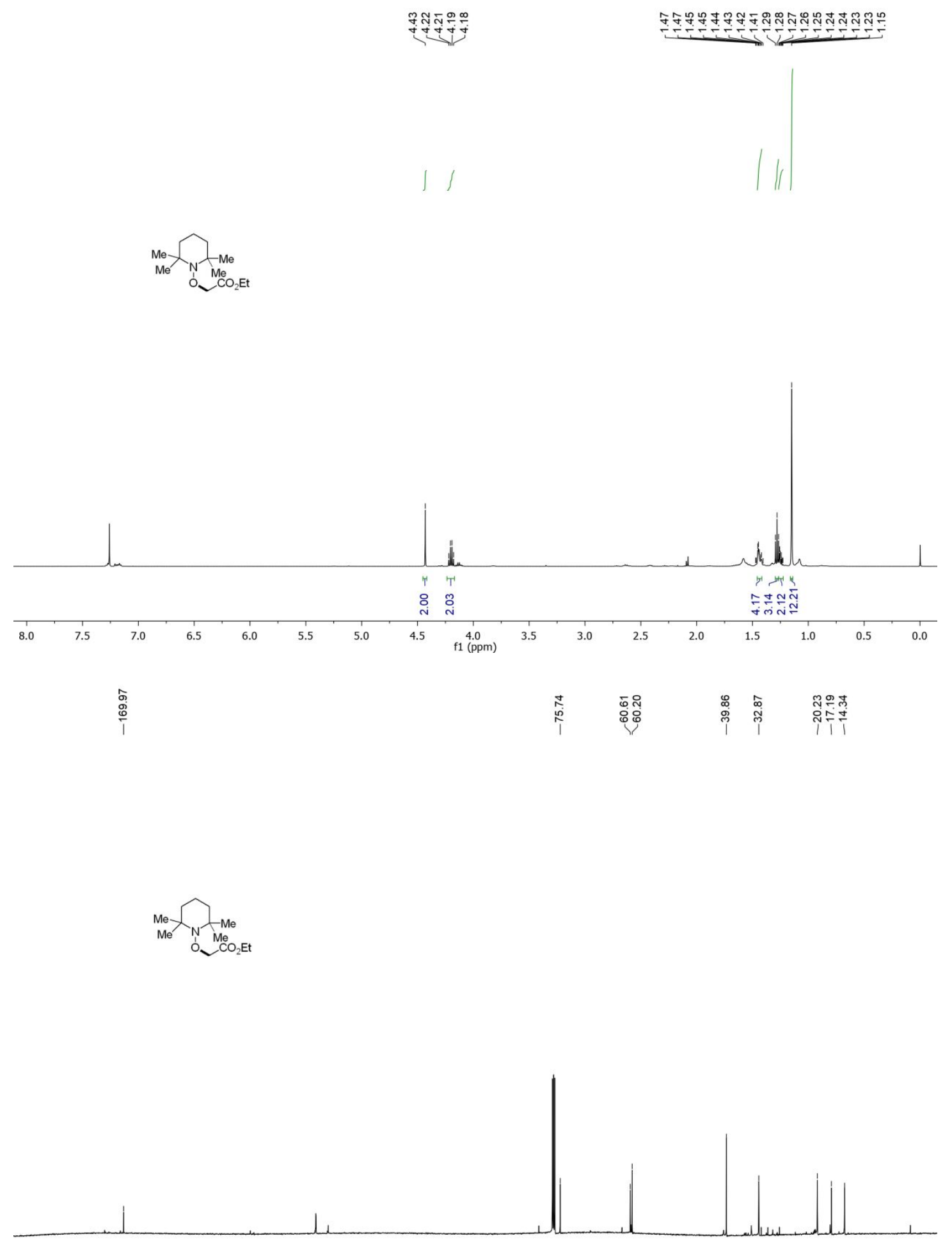

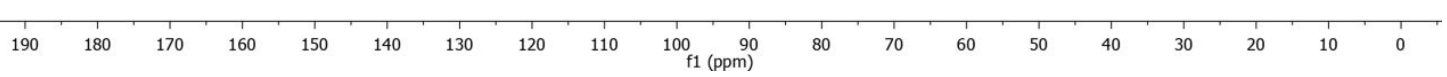


${ }^{1} \mathrm{H}$ NMR (400 MHz, $\left.\mathrm{CDCl}_{3}\right)$ and ${ }^{13} \mathrm{C}$ NMR (125 $\left.\mathrm{MHz}, \mathrm{CDCl}_{3}\right)$ spectra for 4r

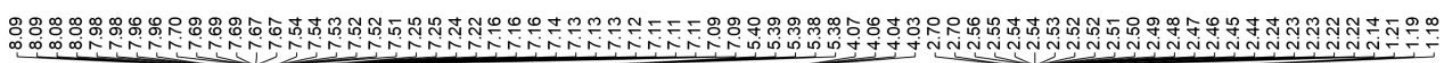
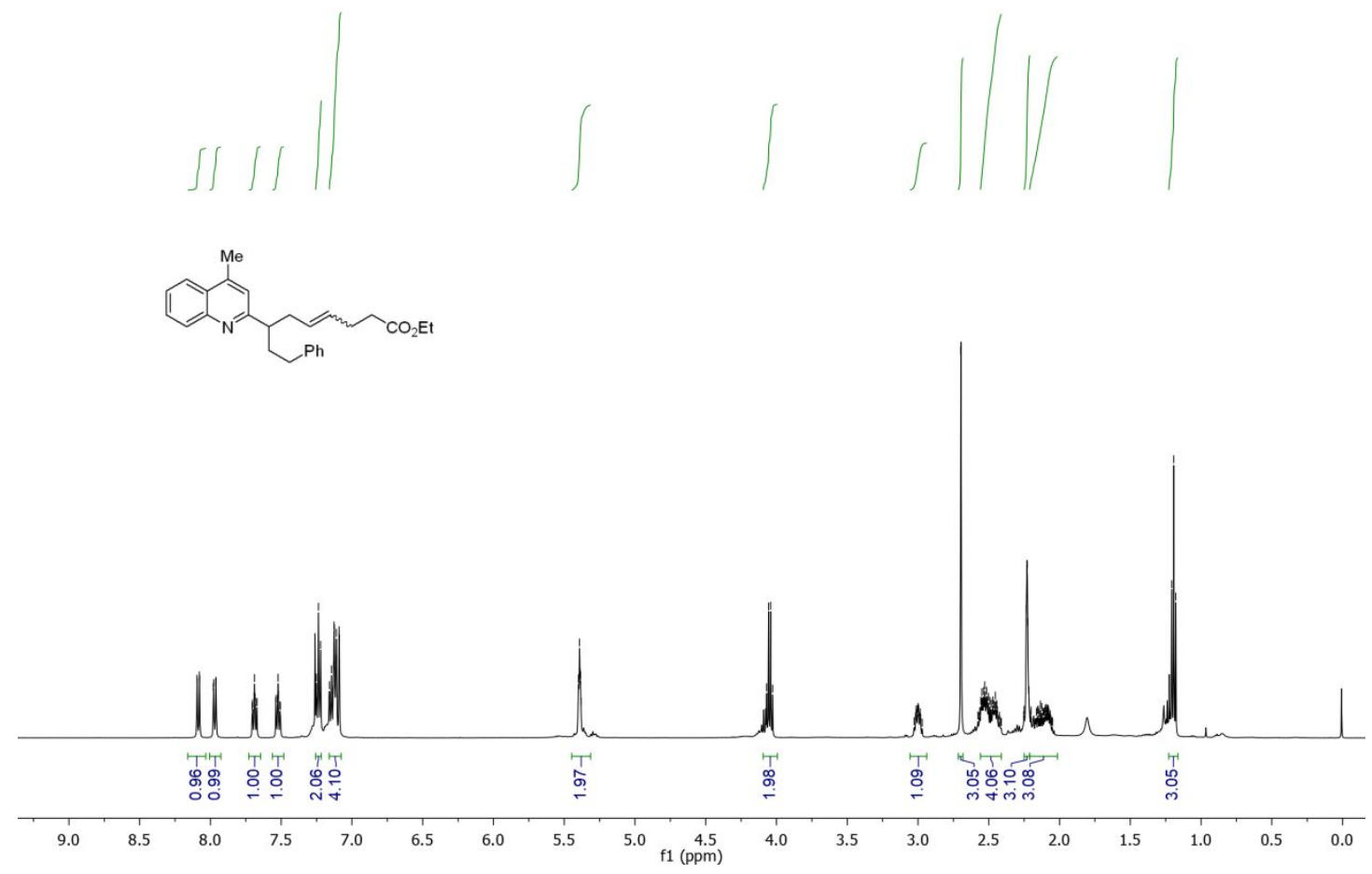

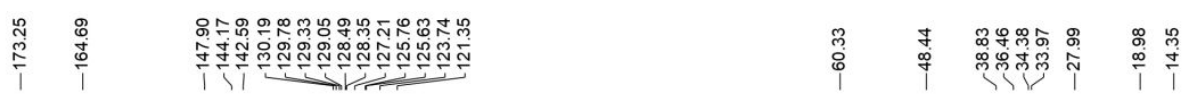

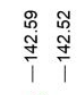

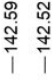
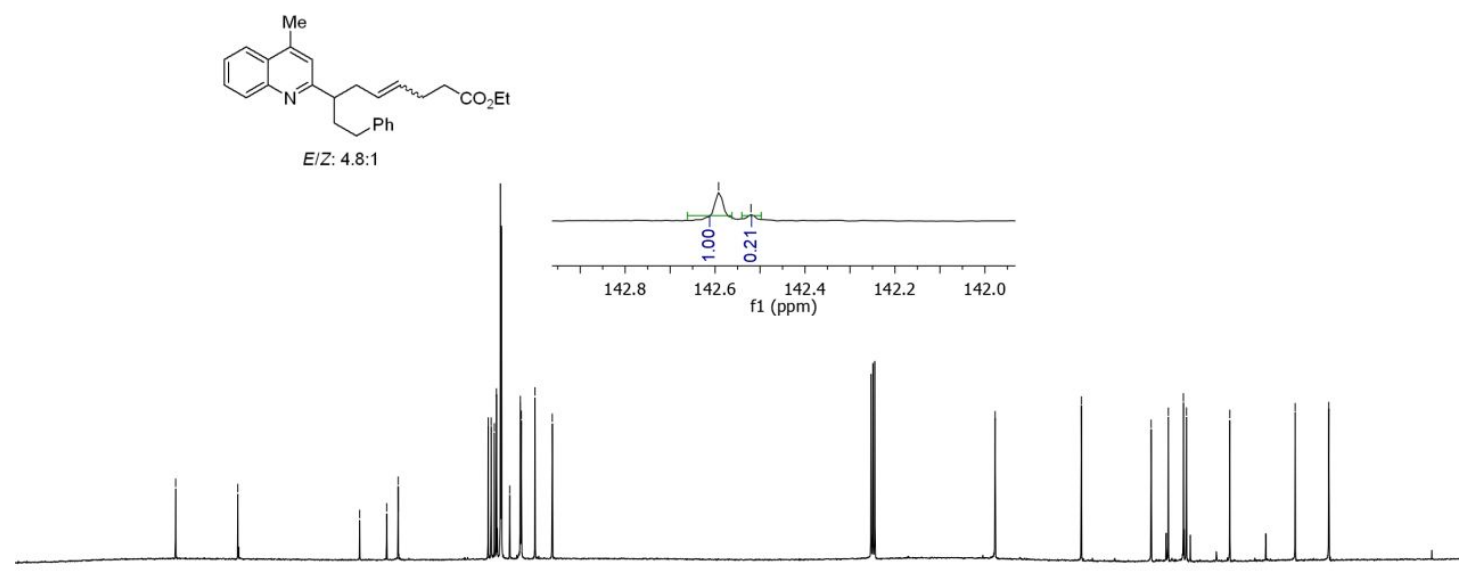

19

16

$50 \quad 140$

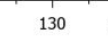

110

${ }_{\mathrm{f} 1(\mathrm{ppm})}^{100}$ 


\section{HPLC spectrum of products}

\section{HPLC spectra for 40}

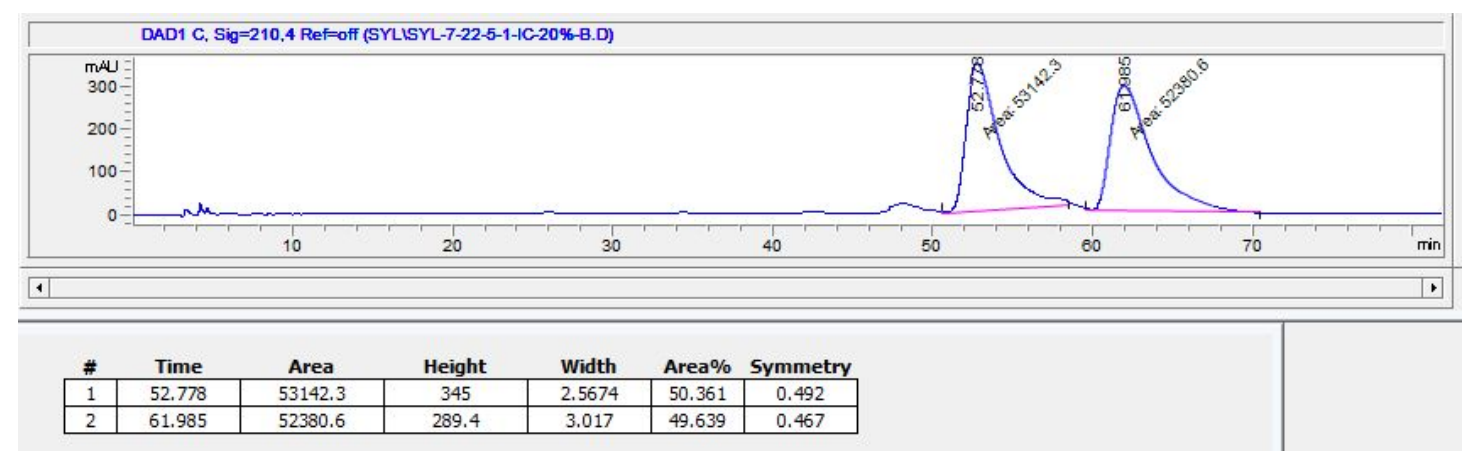

\section{HPLC spectra for $7 \mathbf{i}$}

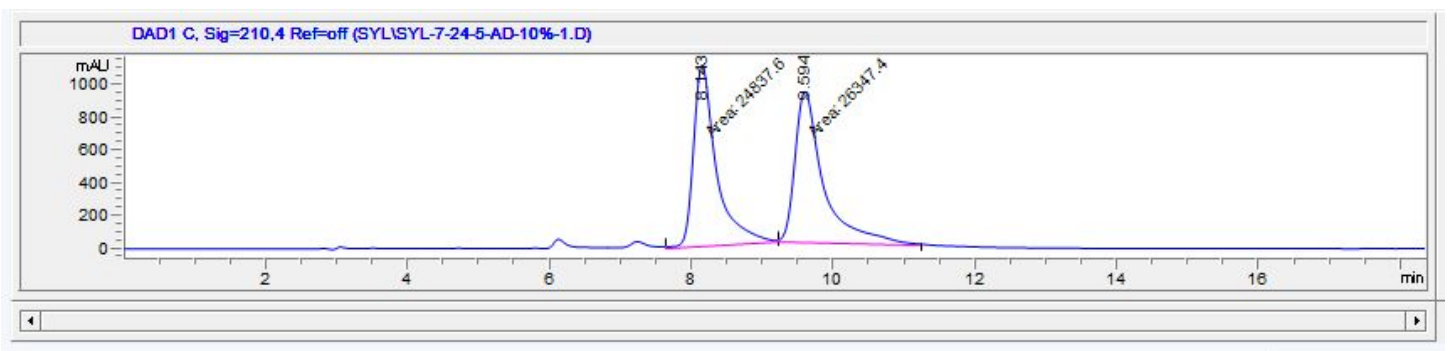

\begin{tabular}{|c|c|c|c|c|c|c|}
\hline \# & Time & Area & Height & Width & Area\% & Symmetry \\
\hline 1 & 8.143 & 24837.6 & 1100.4 & 0.3762 & 48.525 & 0.573 \\
\hline 2 & 9.594 & 26347.4 & 918.5 & 0.4781 & 51.475 & 0.492 \\
\hline
\end{tabular}

